\title{
Catalytic asymmetric Michael reaction of methyl alkynyl ketone catalyzed by diphenylprolinol silyl ether
}

\author{
Nariyoshi Umekubo, Yujiro Hayashi* \\ Department of Chemistry, Graduate School of Science, Tohoku University 6-3 Aramaki-Aza Aoba-ku, \\ Sendai, Miyagi 980-8579, Japan \\ SUPPORTING INFORMATION \\ Experimental procedures and Characterization data
}

Table of Contents

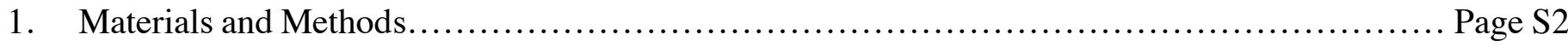

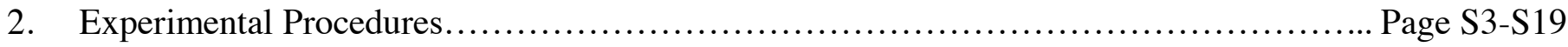

2. 1. Typical procedure of asymmetric Michael reaction using alkynyl ketone................... Page S3

2. 2. Compounds information.................................................................................. Page S3- S7

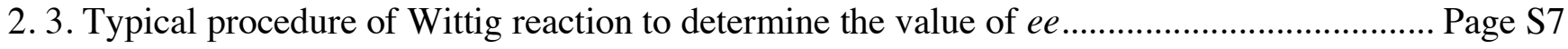

2. 4. Compounds information....................................................................................... Page S7-S12

2. 5. One-pot procedure of providing compound $\mathbf{1 0}$ from compound $\mathbf{1}$ and compound $\mathbf{2 g}$... Page S12-S13

2. 6. One-pot procedure of providing compound 6 from compound 10.................Page S13-S14

2. 7. Lindlar reduction of compound 6 to determine its absolute configuration............. Page S14- S15

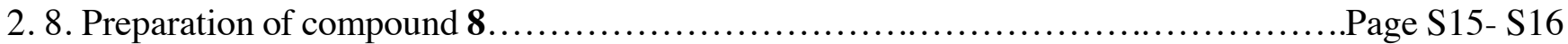

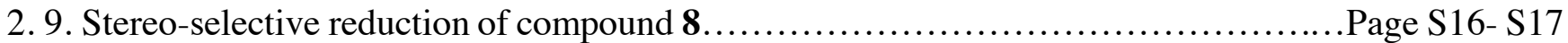

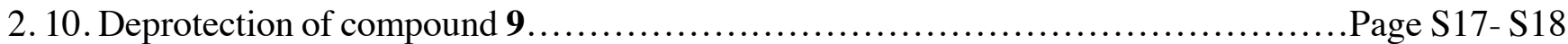

2. 11. Oxidation of compound SI-1 ........................................... Sage S18- S19

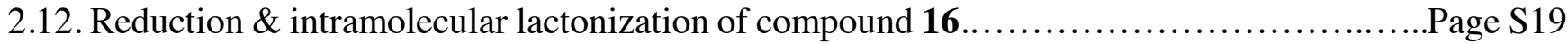

2.13. A side-reaction in asymmetric Michael reaction..............................Page S19- S20

2.14. Compounds information about side-product.................................Page S20- S21

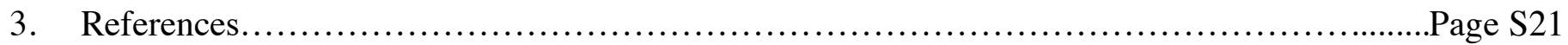

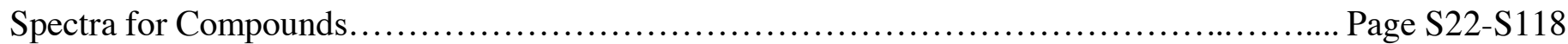




\section{Materials and Methods}

\section{General Methods.}

General Remarks: All reactions were carried out under argon atmosphere and monitored by thin-layer chromatography using Merck 60 F254 precoated silica gel plates (0.25 mm thickness). Specific optical rotations were measured using a JASCO P-1020 polarimeter and a JASCO DIP-370 polarimeter. FT-IR spectra were recorded on a JASCO FT/IR-410 spectrometer and a Perkin Elmer spectrum BX FT-IP spectrometer. ${ }^{1} \mathrm{H}$ and ${ }^{13} \mathrm{C}$ NMR spectra were recorded on an Agilent-400 MR (400 MHz for ${ }^{1} \mathrm{H}$ NMR, $100 \mathrm{M}$ $\mathrm{Hz}$ for ${ }^{13} \mathrm{C} \mathrm{NMR}$ ) instrument. Data for ${ }^{1} \mathrm{H}$ NMR are reported as chemical shift ( $\left.\delta \mathrm{ppm}\right)$, integration multiplicity $(\mathrm{s}=$ singlet, $\mathrm{d}=$ doublet, $\mathrm{t}=$ triplet, $\mathrm{q}=$ quartet, quintet $=$ quin, septet $=\mathrm{sep}, \mathrm{dd}=$ doublet of doublets, $\mathrm{ddd}=$ doublet of doublet of doublets, $\mathrm{dt}=$ double of triplets, $\mathrm{td}=$ triplet of doublets, $\mathrm{m}=$ multiplet, brs $=$ broad singlet), coupling constant (Hz), Data for ${ }^{13} \mathrm{C}$ NMR are reported as chemical shift. High resolution ESI-TOF mass spectra were measured by Themo Orbi-trap instrument. HPLC analysis was performed on a HITACHI Elite LaChrom Series HPLC, UV detection monitored at appropriate wavelength respectively, using CHIRALPACK ${ }^{\circledR}$ IG $(0.46 \mathrm{~cm} \times 25 \mathrm{~cm})$. 


\section{Experimental Procedures}

\subsection{Typical procedure of asymmetric Michael reaction using alkynyl ketone}

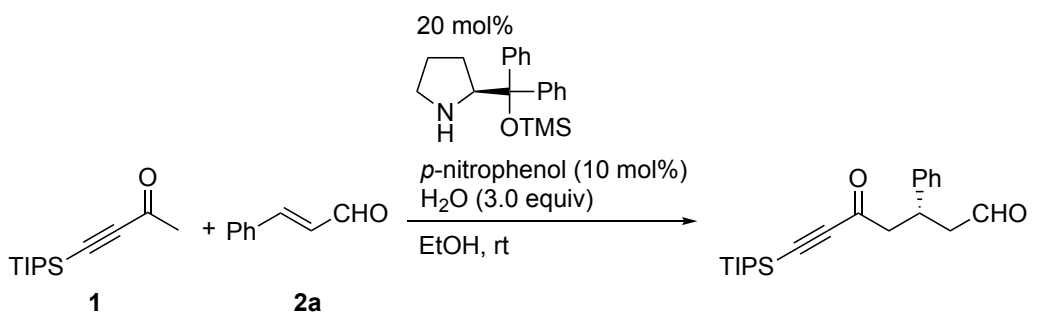

To a solution of cinnamaldehyde $\mathbf{1}(23.5 \mathrm{mg}, 0.18 \mathrm{mmol})$ and 4-(triisopropylsilyl)but-3-yn-2-one $\mathbf{2 a}(33.7$ $\mathrm{mg}, 0.15 \mathrm{mmol})$ in EtOH $(600 \mu \mathrm{L}), \mathrm{H}_{2} \mathrm{O}(8.1 \mu \mathrm{L}, 0.45 \mathrm{mmol})$, diphenylprolinol silyl ether $(9.7 \mathrm{mg}, 0.030$ mmol), p-nitrophenol $(2.1 \mathrm{mg}, 0.015 \mathrm{mmol})$ were added at room temperature. After stirred the reaction mixture at this temperature for 3 hours, its solvent was removed under reduced pressure. The residue was directly purified by column chromatography on silica gel $(n$-Hexane: EtOAc $=12: 1)$ to give the target compound (37.1 mg, $0.104 \mathrm{mmol})$ in $69 \%$ yield.

\subsection{Compounds information}

\section{(R)-5-Oxo-3-phenyl-7-(triisopropylsilyl)hept-6-ynal (3a)}<smiles>O=CC[C@H](CC(=O)C#CSP)c1ccccc1</smiles>

Yield: $69 \%(36.8 \mathrm{mg})$

Physical State: Yellow oil

${ }^{1} \mathbf{H}$ NMR $\left(\mathbf{C D C l}_{3}\right) \delta$ 1.05-1.15 (m, 21H), 2.79-2.82 (m, 2H), $2.95(\mathrm{~d}, J=7.2 \mathrm{~Hz}, 2 \mathrm{H}), 3.90$ (quin, $\left.J=7.2,1 \mathrm{H}\right)$, 7.20-7.24 (m, 3H), 7.29-7.33 (m, 2H), $9.66(\mathrm{t}, J=2.0 \mathrm{~Hz}, 1 \mathrm{H})$

${ }^{13} \mathbf{C ~ N M R}\left(\mathbf{C D C l}_{3}\right) \delta$ 200.4, 185.1, 142.1 , 128.9, 128.9, 127.3, 127.1, 127.1, 104.0, 96.7, 51.6, 49.3, 35.9, 18.5, $18.5,18.5,18.5,18.5,18.5,10.9,10.9,10.9$

HRMS (ESI): [M+Na] $]^{+}$calcd for $\mathrm{C}_{22} \mathrm{H}_{32} \mathrm{O}_{2} \mathrm{SiNa}$ : 379.2064, found: 379.2064

IR(neat) $v 2145,1726,1677,1463,1386,1222,1116,1073,997,883,7000,679,586,457,410 \mathrm{~cm}^{-1}$

$[\alpha]_{\mathrm{D}}{ }^{26}-20.1\left(c\right.$ 2.0, $\left.\mathrm{CHCl}_{3}\right)$

$\mathbf{R}_{\mathbf{f}}(n$-Hexane: EtOAc $=3: 1$, color reagent: Hanessian's stain reagent $): 0.40$ 


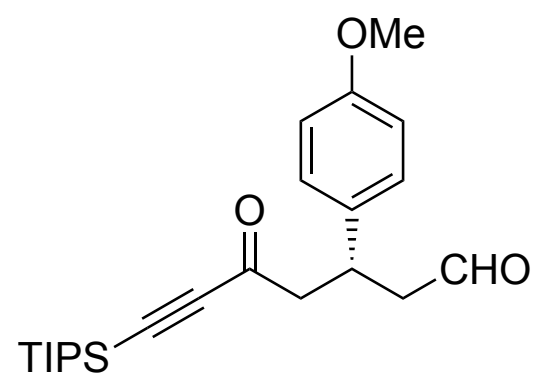

Yield: $68 \%(39.3 \mathrm{mg})$

Physical State: Yellow oil

${ }^{1} \mathbf{H}$ NMR $\left(\mathbf{C D C l}_{3}\right) \delta$ 1.06-1.15 (m, 21H), 2.75-2.78 (m, 2H), $2.92(\mathrm{~d}, J=7.2 \mathrm{~Hz}, 2 \mathrm{H}), 3.77(\mathrm{~s}, 3 \mathrm{H}), 3.85$ (quin, $J=7.2 \mathrm{~Hz}, 1 \mathrm{H}), 6.83(\mathrm{~d}, J=8.8 \mathrm{~Hz}, 2 \mathrm{H}), 7.14(\mathrm{~d}, J=8.8 \mathrm{~Hz}, 2 \mathrm{H}), 9.65(\mathrm{t}, J=2.0 \mathrm{~Hz}, 1 \mathrm{H})$

${ }^{13} \mathbf{C ~ N M R}\left(\mathbf{C D C l}_{3}\right) \delta$ 200.7, 185.3, 158.6, 134.1, 128.3, 128.3, 114.2, 114.2, 104.1, 96.6, 55.2, 51 .9, 49.5, 34.9, $18.5,18.5,18.5,18.5,18.5,18.5,11.0,11.0,11.0$

HRMS (ESI): $[\mathrm{M}+\mathrm{Na}]^{+}$calcd for $\mathrm{C}_{23} \mathrm{H}_{34} \mathrm{O}_{3} \mathrm{SiNa}$ : 409.2169, found: 409.2169

$\operatorname{IR}\left(\right.$ neat) $v 2145,1726,1677,1463,1386,1222,1116,1073,997,883,7000,679,586,457,410 \mathrm{~cm}^{-1}$ $[\alpha]_{\mathrm{D}}{ }^{26}-15.5\left(c 1.5, \mathrm{CHCl}_{3}\right)$

$\mathbf{R}_{\mathbf{f}}(n$-Hexane: EtOAc $=3: 1$, color reagent: Hanessian's stain reagent $): 0.20$

\section{(R)-3-(4-Bromophenyl)-5-oxo-7-(triisopropylsilyl)hept-6-ynal (3c)}

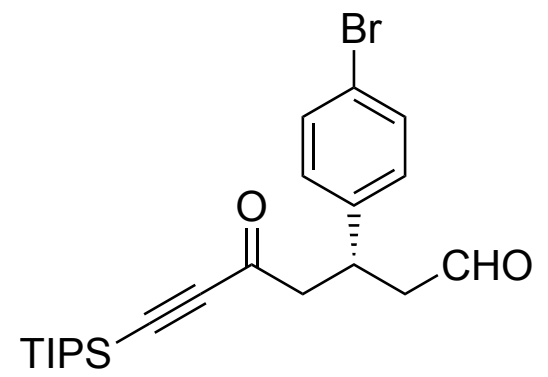

Yield: $61 \%(37.9 \mathrm{mg})$

Physical State: Yellow oil

${ }^{1} \mathrm{H}$ NMR $\left(\mathbf{C D C l}_{3}, \mathbf{4 0 0} \mathrm{MHz}\right) \delta$ 1.06-1.11 (m, 21H), $2.80(\mathrm{dt}, J=1.6,6.8 \mathrm{~Hz}, 2 \mathrm{H}), 2.92(\mathrm{dd}, J=2.4,7.2 \mathrm{~Hz}$, $2 \mathrm{H}), 3.86$ (quin, $J=7.2 \mathrm{~Hz}, 1 \mathrm{H}), 7.11(\mathrm{~d}, J=8.4 \mathrm{~Hz}, 2 \mathrm{H}), 7.42(\mathrm{~d}, J=8.4 \mathrm{~Hz}, 2 \mathrm{H}), 9.66(\mathrm{t}, J=1.2 \mathrm{~Hz}, 1 \mathrm{H})$ ${ }^{13} \mathbf{C ~ N M R}\left(\mathbf{C D C l}_{3}, \mathbf{1 0 0}\right.$ MHz) $\delta$ 199.8, 188.9, 141.2, 132.0, 132.0, 129.1, 129.1, 121.0, 104.0, 91.6, 51.3, 49.2, $34.9,18.5,18.5,18.5,18.5,18.5,18.5,11.0,11.0,11.0$

HRMS (ESI): [M+Na] $]^{+}$calcd for $\mathrm{C}_{22} \mathrm{H}_{31} \mathrm{BrO}_{2} \mathrm{SiNa}$ : 457.1169, found: 457.1172

IR(neat) $v 2145,1726,1677,1489,1463,1118,1074,1011,883,821,681,404 \mathrm{~cm}^{-1}$

$[\alpha]_{\mathrm{D}}{ }^{26}-19.5\left(c 1.7, \mathrm{CHCl}_{3}\right)$

$\mathbf{R}_{\mathbf{f}}(n$-Hexane: EtOAc $=3: 1$, color reagent: Hanessian's stain reagent $): 0.45$ 


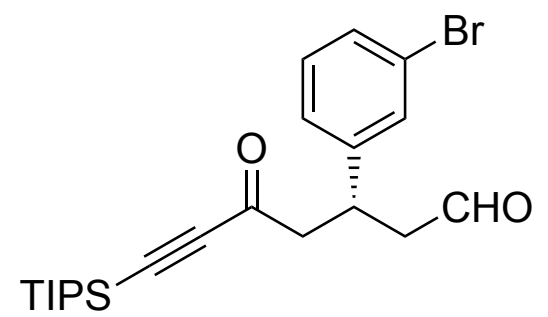

Yield: $64 \%(41.6 \mathrm{mg})$

Physical State: Yellow oil

${ }^{1} \mathbf{H}$ NMR $\left(\mathbf{C D C l}_{3}, \mathbf{4 0 0} \mathbf{M H z}\right) \delta 1.06-1.17(\mathrm{~m}, 21 \mathrm{H}), 2.81(\mathrm{ddd}, J=1.6,5.6,7.2 \mathrm{~Hz}, 2 \mathrm{H}), 2.93(\mathrm{dd}, J=1.2,6.8$ $\mathrm{Hz}, 2 \mathrm{H}$ ), 3.86 (quin, $J=7.2 \mathrm{~Hz}, 1 \mathrm{H}), 7.16-7.18(\mathrm{~m}, 2 \mathrm{H}), 7.34-7.38(\mathrm{~m}, 2 \mathrm{H}), 9.70(\mathrm{t}, J=1.6 \mathrm{~Hz}, 1 \mathrm{H})$

${ }^{13} \mathbf{C ~ N M R}\left(\mathbf{C D C l}_{3}, \mathbf{1 0 0}\right.$ MHz) $\delta$ 199.7, 184.6, 144.6, 130.5, 130.4, 130.3, 126.2, 122.9, 103.9, 97.1, 51.2, 49.1, $35.0,18.5,18.5,18.5,18.5,18.5,18.5,11.0,11.0,11.0$

HRMS (ESI): [M+Na] $]^{+}$calcd for $\mathrm{C}_{22} \mathrm{H}_{31} \mathrm{BrO}_{2} \mathrm{SiNa}$ : 457.1169, found: 457.1173

$\operatorname{IR}\left(\right.$ neat) $v 2145,1726,1677,1568,1463,1428,1221,1117,1074,997,882,784,679,587,440,408 \mathrm{~cm}^{-1}$ $[\alpha]_{\mathrm{D}}{ }^{26}-38.5\left(c 0.50, \mathrm{CHCl}_{3}\right)$

$\mathbf{R}_{\mathbf{f}}(n$-Hexane: EtOAc $=3: 1$, color reagent: Hanessian's stain reagent $): 0.45$

\section{(R)-3-(2-Bromophenyl)-5-oxo-7-(triisopropylsilyl)hept-6-ynal (3e)}

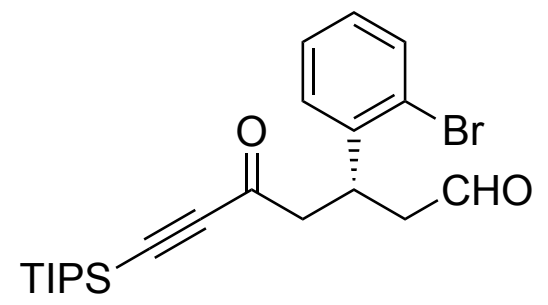

Yield: $70 \%(45.6 \mathrm{mg})$

Physical State: Yellow oil

${ }^{1} \mathbf{H}$ NMR $\left(\mathbf{C D C l}_{3}, 400 \mathrm{MHz}\right) \delta 1.07-1.15(\mathrm{~m}, 21 \mathrm{H}), 2.85(\mathrm{dd}, J=1.6,7.2 \mathrm{~Hz}, 2 \mathrm{H}), 2.94(\mathrm{dd}, J=8.0,17.6 \mathrm{~Hz}$, $1 \mathrm{H}), 3.03(\mathrm{dd}, J=6.0,16.8 \mathrm{~Hz}, 1 \mathrm{H}), 4.37$ (quin, $J=7.2 \mathrm{~Hz}, 1 \mathrm{H}), 7.09(\mathrm{dt}, J=2.0,8.0 \mathrm{~Hz}, 1 \mathrm{H}), 7.21(\mathrm{dd}, J=$ $1.6,8.0 \mathrm{~Hz}, 1 \mathrm{H}), 7.28(\mathrm{dt}, J=1.2,7.6 \mathrm{~Hz}, 1 \mathrm{H}), 7.56(\mathrm{dd}, J=1.2,8.0 \mathrm{~Hz}, 1 \mathrm{H}), 9.68(\mathrm{t}, J=1.6 \mathrm{~Hz}, 1 \mathrm{H})$

${ }^{13} \mathbf{C}$ NMR $\left(\mathbf{C D C l}_{3}, \mathbf{1 0 0}\right.$ MHz) $\delta$ 200.1, 184.8, 140.8, 133.5, 128.6, 128.1, 127.9, 124.3, 103.8, 96.9, 49.8, 47.9, $34.5,18.5,18.5,18.5,18.5,18.5,18.5,11.0,11.0,11.0$

HRMS (ESI): [M+Na] $]^{+}$calcd for $\mathrm{C}_{22} \mathrm{H}_{31} \mathrm{BrO}_{2} \mathrm{SiNa}$ : 457.1169, found: 457.1170

IR(neat) $v 2146,1726,1676,1470,1223,1110,1022,883,755,681,585,450 \mathrm{~cm}^{-1}$

$[\alpha]_{\mathrm{D}}{ }^{26}+3.40\left(c 1.9, \mathrm{CHCl}_{3}\right)$

$\mathbf{R}_{\mathbf{f}}(n$-Hexane: EtOAc $=3: 1$, color reagent: Hanessian's stain reagent $): 0.45$ 


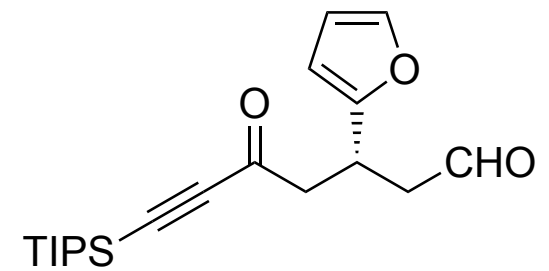

Yield: $78 \%(40.8 \mathrm{mg})$

Physical State: Yellow oil

${ }^{1} \mathrm{H}$ NMR $\left(\mathbf{C D C l}_{3}, 400 \mathrm{MHz}\right) \delta 1.04-1.17(\mathrm{~m}, 21 \mathrm{H}), 2.79(\mathrm{dt}, J=1.6,5.6 \mathrm{~Hz}, 2 \mathrm{H}), 2.90(\mathrm{dd}, J=7.6,17.2 \mathrm{~Hz}$, $1 \mathrm{H}), 3.02(\mathrm{dd}, J=6.8,16.8 \mathrm{~Hz}, 1 \mathrm{H}), 3.99$ (quin, $J=7.6 \mathrm{~Hz}, 1 \mathrm{H}), 6.06(\mathrm{~d}, J=3.2 \mathrm{~Hz}, 1 \mathrm{H}), 6.26(\mathrm{dd}, J=1.6$, $3.2 \mathrm{~Hz}, 1 \mathrm{H}), 7.30(\mathrm{t}, J=0.80 \mathrm{~Hz}, 1 \mathrm{H}), 9.72(\mathrm{t}, J=2.0 \mathrm{~Hz}, 1 \mathrm{H})$

${ }^{13} \mathbf{C}$ NMR (CDCl 3 , 100 MHz) $\delta$ 200.1, 184.7, 154.9, 141.7, 110.2, 105.9, 103.8, 96.9, 48.7, 46.6, 29.0, 18.5, $18.5,18.5,18.5,18.5,18.5,10.9,10.9,10.9$

HRMS (ESI): $[\mathrm{M}+\mathrm{Na}]^{+}$calcd for $\mathrm{C}_{20} \mathrm{H}_{30} \mathrm{O}_{3} \mathrm{SiNa}: 369.1856$, found: 369.1858

$\operatorname{IR}\left(\right.$ neat) $v 2146,1727,1679,1464,1218,1115,1072,1015,883,734,681,597 \mathrm{~cm}^{-1}$

$[\alpha]_{\mathrm{D}}{ }^{26}-4.41\left(c 1.5, \mathrm{CHCl}_{3}\right)$

$\mathbf{R}_{\mathbf{f}}(n$-Hexane: EtOAc $=3: 1$, color reagent: Hanessian's stain reagent $): 0.20$

(R)-3-(Dimethyl(phenyl)silyl)-5-oxo-7-(triisopropylsilyl)hept-6-ynal (3g)

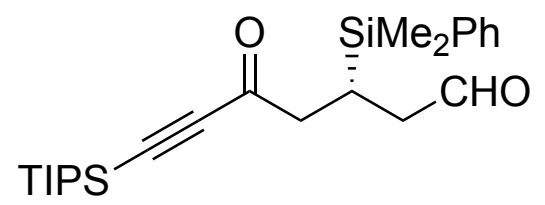

Yield: $71 \%(44.1 \mathrm{mg})$

Physical State: Yellow oil

${ }^{1} \mathrm{H}$ NMR $\left(\mathbf{C D C l}_{3}, 400 \mathrm{MHz}\right) \delta 0.33(\mathrm{~s}, 6 \mathrm{H}), 1.08-1.12(\mathrm{~m}, 21 \mathrm{H}), 2.01-2.16(\mathrm{~m}, 1 \mathrm{H}), 2.34$ (ddd, $J=2.0,8.0$, $17.6 \mathrm{~Hz}, 1 \mathrm{H}), 2.49(\mathrm{dd}, J=9.6,16.8 \mathrm{~Hz}, 1 \mathrm{H}), 2.50(\mathrm{ddd}, J=1.6,5.6,17.6 \mathrm{~Hz}, 1 \mathrm{H}), 2.69$ (dd, $J=4.8,16.8 \mathrm{~Hz}$, $1 \mathrm{H}), 7.34-7.39(\mathrm{~m}, 3 \mathrm{H}), 7.47-7.50(\mathrm{~m}, 2 \mathrm{H}), 9.62(\mathrm{t}, J=1.2 \mathrm{~Hz}, 1 \mathrm{H})$

${ }^{13} \mathbf{C ~ N M R}\left(\mathbf{C D C l}_{3}, \mathbf{1 0 0} \mathbf{M H z}\right) \delta$ 201.5, 187.1, 136.2, 133.9, 133.9, 129.5, 128.0, 128.0, 104.0, 96.4 46.0, 44.1, $18.5,18.5,18.5,18.5,18.5,18.5,15.4,11.0,11.0,11.0,-4.37,-4.46$

HRMS (ESI): $[\mathrm{M}+\mathrm{Na}]^{+}$calcd for $\mathrm{C}_{24} \mathrm{H}_{38} \mathrm{O}_{2} \mathrm{Si}_{2} \mathrm{Na}$ : 437.2303, found: 437.2303

IR(neat) $v 2145,1725,1677,1462,1427,1253,1212,1112,1069,997,883,836,778,736,701,681,471 \mathrm{~cm}^{-}$

$[\alpha]_{\mathrm{D}}{ }^{26}-5.02\left(c 1.5, \mathrm{CHCl}_{3}\right)$

$\mathbf{R}_{\mathbf{f}}(n$-Hexane: EtOAc $=3: 1$, color reagent: Hanessian's stain reagent $): 0.50$ 


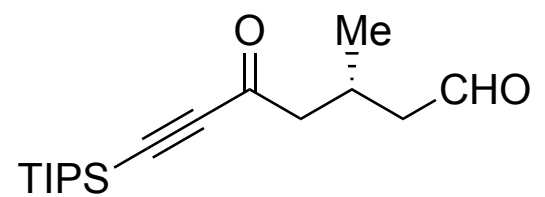

Yield: $55 \%(24.3 \mathrm{mg})$

Physical State: Colorless oil

${ }^{1} \mathrm{H}$ NMR $\left(\mathbf{C D C l}_{3}, \mathbf{4 0 0} \mathrm{MHz}\right) \delta 1.04(\mathrm{~d}, J=6.8 \mathrm{~Hz}, 3 \mathrm{H}), 1.08-1.16(\mathrm{~m}, 21 \mathrm{H}), 2.35(\mathrm{ddd}, J=2.4,8.0,17.2 \mathrm{~Hz}$, $1 \mathrm{H}), 2.48-2.60(\mathrm{~m}, 2 \mathrm{H}), 2.60-2.66(\mathrm{dd}, J=6.4,16.4 \mathrm{~Hz}, 1 \mathrm{H}), 2.72$ (quin, $J=6.8 \mathrm{~Hz}, 1 \mathrm{H}), 9.75(\mathrm{t}, J=1.6 \mathrm{~Hz}$, $1 \mathrm{H})$

${ }^{13} \mathbf{C}$ NMR $\left(\mathbf{C D C l}_{3}, \mathbf{1 0 0} \mathbf{M H z}\right) \delta 201.3,186.2,104.2,96.2,51.9,50.0,24.6,20.0,18.5,18.5,18.5,18.5,18.5$, $18.5,10.8,10.8,10.8$

HRMS (ESI): $[\mathrm{M}+\mathrm{Na}]^{+}$calcd for $\mathrm{C}_{17} \mathrm{H}_{30} \mathrm{O}_{2} \mathrm{SiNa}: 317.1907$, found: 317.1907

IR(neat) $v 2145,1726,1673,1463,1163,1072,996,918,883,701,680,505 \mathrm{~cm}^{-1}$

$[\alpha]_{\mathrm{D}}{ }^{26}+20.6\left(c 0.20, \mathrm{CHCl}_{3}\right)$

$\mathbf{R}_{\mathbf{f}}(n$-Hexane: EtOAc $=3: 1$, color reagent: Hanessian's stain reagent): 0.60

\subsection{Typical procedure of Wittig reaction to determine the value of $e e$}

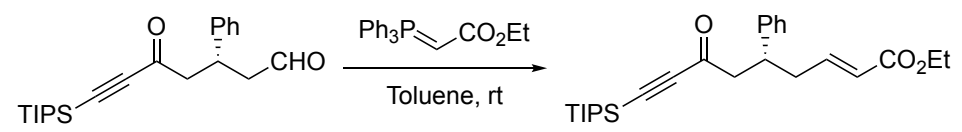

To a solution of aldehyde $(37.1 \mathrm{mg}, 0.10 \mathrm{mmol})$ in toluene $(300 \mu \mathrm{L}), \mathrm{Ph}_{3} \mathrm{P}=\mathrm{CHCO}_{2} \mathrm{Et}(69.6 \mathrm{mg}, 0.20 \mathrm{mmol})$ was added at room temperature. After stirring the reaction mixture at this temperature for 1 hour, the reaction mixture was directly purified by column chromatography on silica gel $(n$-Hexane: EtOAc $=3: 1)$ to give the target compound.

The enantiomeric ratio was determined by HPLC using CHIRALPACK ${ }^{\circledR}$ IG $(n$-Hexane: $i$-PrOH $=66: 1$; flow rate $1.0 \mathrm{ml} / \mathrm{min}$, major isomer $\mathrm{t}_{\mathrm{R}}=12.7 \mathrm{~min}$, minor isomer $\left.\mathrm{t}_{\mathrm{R}}=24.9 \mathrm{~min}\right)(95 \%$ ee $)$.

\subsection{Compounds information}

Ethyl $(R, E)-7-0 x 0-5-p h e n y l-9-(t r i i s o p r o p y l s i l y l) n o n-2-e n-8-y n o a t e$<smiles>CCOC(=O)/C=C/C[C@@H](CC(=O)C#CS)c1ccccc1</smiles>

Yield: $73 \%(31.2 \mathrm{mg})$ 
Physical State: Yellow oil

${ }^{1} \mathrm{H}$ NMR $\left(\mathbf{C D C l}_{3}, 400 \mathrm{MHz}\right) \delta$ 0.94-1.17 (m, 21H), $1.25(\mathrm{t}, J=7.2 \mathrm{~Hz}, 3 \mathrm{H}), 2.51-2.60(\mathrm{~m}, 2 \mathrm{H}), 2.90(\mathrm{~d}, J=$ $3.6 \mathrm{~Hz}, 2 \mathrm{H}), 3.48$ (quin, $J=7.2 \mathrm{~Hz}, 1 \mathrm{H}), 4.14(\mathrm{q}, J=7.2 \mathrm{~Hz}, 2 \mathrm{H}), 5.78(\mathrm{~d}, J=15.6 \mathrm{~Hz}, 1 \mathrm{H}), 6.78(\mathrm{dd}, J=7.6$, $15.6 \mathrm{~Hz}, 1 \mathrm{H}), 7.17-7.23(\mathrm{~m}, 2 \mathrm{H}), 7.26-7.32(\mathrm{~m}, 3 \mathrm{H})$

${ }^{13} \mathrm{C}$ NMR $\left(\mathrm{CDCl}_{3}, \mathbf{1 0 0} \mathrm{MHz}\right) \delta 185.6,166.2,145.6,142.3,128.7,128.7,127.4,127.4,127.0,123.5,104.1$, $96.5,60.2,51.5,40.7,38.6,18.5,18.5,18.5,18.5,18.5,18.5,14.2,11.0,11.0,11.0$

HRMS (ESI): $[\mathrm{M}+\mathrm{Na}]^{+}$calcd for $\mathrm{C}_{26} \mathrm{H}_{38} \mathrm{O}_{3} \mathrm{SiNa}$ : 449.2482, found: 449.2480

IR(neat) $v$ 2145, 1721, 1677, 1463, 1367, 1264, 1207, 1160, 1109, 1075 1041, 997, 883, 838, 762, 700, 679 $\mathrm{cm}^{-1}$

$[\alpha]_{\mathrm{D}}{ }^{26}-7.14\left(c 1.8, \mathrm{CHCl}_{3}\right)$

$\mathbf{R}_{\mathbf{f}}(n$-Hexane: EtOAc =3:1, color reagent: Hanessian's stain reagent): 0.40

The enantiomeric ratio was determined by HPLC using CHIRALPACK ${ }^{\circledR}$ IG $(n$-Hexane: $i$-PrOH $=66: 1$; flow rate $1.0 \mathrm{ml} / \mathrm{min}$, major isomer $t_{R}=12.7 \mathrm{~min}$, minor isomer $\left.t_{R}=24.9 \mathrm{~min}\right)(95 \% e e)$.

Ethyl $(R, E)-5-(4-m e t h o x y p h e n y l)-7-0 x 0-9-(t r i i s o p r o p y l s i l y l) n o n-2-e n-8-y n o a t e$

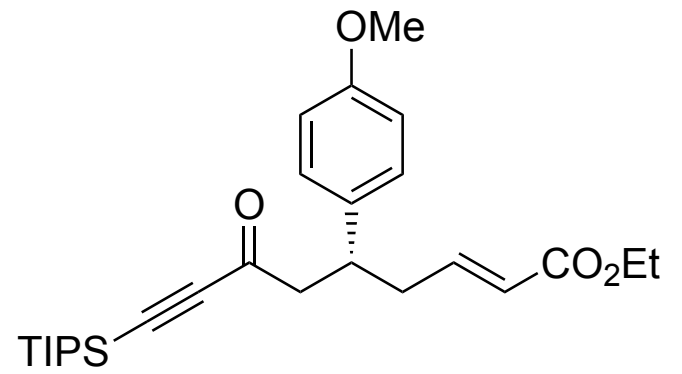

Yield: $80 \%(36.5 \mathrm{mg})$

Physical State: Yellow oil

${ }^{1} \mathbf{H}$ NMR $\left(\mathbf{C D C l}_{3}, 400 \mathrm{MHz}\right) \delta$ 1.04-1.17 (m, 21H), $1.25(\mathrm{t}, J=7.2 \mathrm{~Hz}, 3 \mathrm{H}), 2.45-2.58(\mathrm{~m}, 2 \mathrm{H}), 2.85(\mathrm{~d}, J=$ $7.6 \mathrm{~Hz}, 2 \mathrm{H}), 3.43$ (quin, $J=7.2 \mathrm{~Hz}, 1 \mathrm{H}), 3.78(\mathrm{~s}, 3 \mathrm{H}), 4.14(\mathrm{q}, J=7.2 \mathrm{~Hz}, 2 \mathrm{H}), 5.76(\mathrm{~d}, J=15.6 \mathrm{~Hz}, 1 \mathrm{H}), 6.79$ $(\mathrm{td}, J=7.2,15.6 \mathrm{~Hz}, 1 \mathrm{H}), 6.82(\mathrm{~d}, J=8.8 \mathrm{~Hz}, 2 \mathrm{H}), 7.09(\mathrm{~d}, J=8.8 \mathrm{~Hz}, 2 \mathrm{H})$

${ }^{13} \mathbf{C}$ NMR (CDCl 3 , 100 MHz) $\delta 185.8,166.2,158.4,145.8,134.3,128.3,128.3,123.4,114.1,114.1,104.2$, $96.5,60.2,55.2,51.8,40.0,38.8,18.5,18.5,18.5,18.5,18.5,18.5,14.2,11.0,11.0,11.0$

HRMS (ESI): $[\mathrm{M}+\mathrm{Na}]^{+}$calcd for $\mathrm{C}_{27} \mathrm{H}_{40} \mathrm{O}_{4} \mathrm{SiNa}: 479.2588$, found: 479.2593

IR(neat) $v 2146,1718,1675,1513,1458,1251,1038,827,419 \mathrm{~cm}^{-1}$

$[\alpha]_{\mathrm{D}}{ }^{26}-2.84\left(c 0.20, \mathrm{CHCl}_{3}\right)$

$\mathbf{R}_{\mathbf{f}}(n$-Hexane: EtOAc $=3: 1$, color reagent: Hanessian's stain reagent): 0.20

The enantiomeric ratio was determined by HPLC using CHIRALPACK ${ }^{\circledR}$ IG $(n$-Hexane: $i$-PrOH $=66: 1$; flow rate $1.0 \mathrm{ml} / \mathrm{min}$, major isomer $t_{R}=20.6 \mathrm{~min}$, minor isomer $\left.t_{R}=39.3 \mathrm{~min}\right)(95 \% e e)$. 


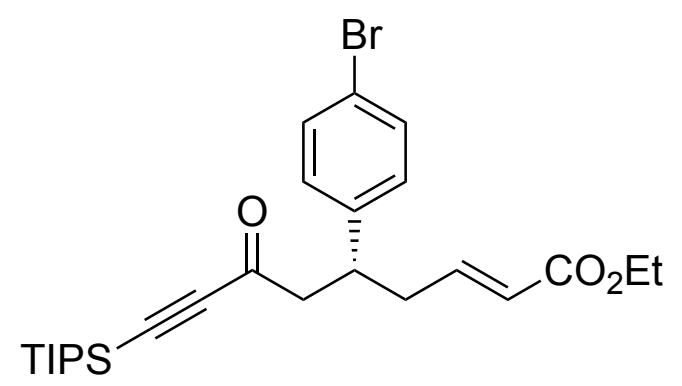

Yield: $81 \%(40.8 \mathrm{mg})$

Physical State: Yellow oil

${ }^{1} \mathrm{H}$ NMR $\left(\mathbf{C D C l}_{3}, 400 \mathrm{MHz}\right) \delta 1.06-1.14(\mathrm{~m}, 21 \mathrm{H}), 1.26(\mathrm{t}, J=7.2 \mathrm{~Hz}, 3 \mathrm{H}), 2.50-2.59(\mathrm{~m}, 2 \mathrm{H}), 2.87(\mathrm{dd}, J=$ $0.80,8.0 \mathrm{~Hz}, 2 \mathrm{H}), 3.43$ (quin, $J=7.2 \mathrm{~Hz}, 1 \mathrm{H}), 4.15(\mathrm{t}, J=7.2 \mathrm{~Hz}, 2 \mathrm{H}), 5.76(\mathrm{~d}, J=15.6 \mathrm{~Hz}, 1 \mathrm{H}), 6.74(\mathrm{td}, J$ $=7.2,15.6 \mathrm{~Hz}, 1 \mathrm{H}), 7.05(\mathrm{~d}, J=8.4 \mathrm{~Hz}, 2 \mathrm{H}), 7.42(\mathrm{~d}, J=8.4 \mathrm{~Hz}, 2 \mathrm{H})$

${ }^{13} \mathrm{C}$ NMR $\left(\mathrm{CDCl}_{3}, \mathbf{1 0 0} \mathrm{MHz}\right) \delta 185.1,166.1,145.0,141.2,131.9,131.9,129.1,129.1,123.8,120.8,104.1$, $96.8,60.3,51.3,40.2,38.4,18.5,18.5,18.5,18.5,18.5,18.5,14.2,11.0,11.0,11.0$

HRMS (ESI): [M+Na] $]^{+}$calcd for $\mathrm{C}_{26} \mathrm{H}_{37} \mathrm{BrO}_{3} \mathrm{SiNa}$ : 527.1588, found: 527.1589

$\operatorname{IR}($ neat $) v 2145,1720,1677,1464,1206,1101,882,679 \mathrm{~cm}^{-1}$

$[\alpha]_{\mathrm{D}}{ }^{26}+2.65\left(c 1.0, \mathrm{CHCl}_{3}\right)$

$\mathbf{R}_{\mathbf{f}}(n$-Hexane: EtOAc $=3: 1$, color reagent: Hanessian's stain reagent): 0.45

The enantiomeric ratio was determined by HPLC using CHIRALPACK ${ }^{\circledR} \mathrm{IG}(n$-Hexane: $i$-PrOH $=66: 1$; flow rate $1.0 \mathrm{ml} / \mathrm{min}$, major isomer $t_{R}=12.7 \mathrm{~min}$, minor isomer $\left.t_{R}=20.3 \mathrm{~min}\right)(94 \% e e)$.

Ethyl $(R, E)-5-(3-b r o m o p h e n y l)-7-o x o-9-(t r i i s o p r o p y l s i l y l) n o n-2-e n-8-y n o a t e$<smiles>CCOC(=O)/C=C/C[C@@H](CC(=O)C#CSP)c1cccc(Br)c1</smiles>

Yield: $78 \%(39.3 \mathrm{mg})$

Physical State: Yellow oil

${ }^{1} \mathrm{H}$ NMR $\left(\mathbf{C D C l}_{3}, 400 \mathrm{MHz}\right) \delta$ 1.07-1.15 (m, 21H), $1.26(\mathrm{t}, J=7.2 \mathrm{~Hz}, 3 \mathrm{H}), 2.46-2.59(\mathrm{~m}, 2 \mathrm{H}), 2.87(\mathrm{~d}, J=$ $7.2 \mathrm{~Hz}, 2 \mathrm{H}), 3.43$ (quin, $J=7.2 \mathrm{~Hz}, 1 \mathrm{H}), 4.15(\mathrm{q}, J=7.2 \mathrm{~Hz}, 2 \mathrm{H}), 5.78(\mathrm{~d}, J=15.6 \mathrm{~Hz}, 1 \mathrm{H}), 6.75(\mathrm{td}, J=7.6$, $15.6 \mathrm{~Hz}, 1 \mathrm{H}), 7.10(\mathrm{~d}, J=7.6 \mathrm{~Hz}, 1 \mathrm{H}), 7.17(\mathrm{t}, J=8.0 \mathrm{~Hz}, 1 \mathrm{H}), 7.33-7.36(\mathrm{~m}, 2 \mathrm{H})$

${ }^{13} \mathbf{C}$ NMR (CDCl 3 , 100 MHz) $\delta 185.0,166.1,145.0,144.7,130.4,130.3,130.2,126.2,123.9,122.8,104.0$, $96.9,60.3,51.2,40.3,38.4,18.7,18.7,18.5,18.5,18.4,18.4,14.2,11.0,11.0,11.0$

HRMS (ESI): [M+Na] $]^{+}$calcd for $\mathrm{C}_{26} \mathrm{H}_{37} \mathrm{BrO}_{3} \mathrm{SiNa}$ : 527.1588, found: 527.1586

IR(neat) $v 2146,1719,1677,1464,1367,1270,1206,1113,882,680 \mathrm{~cm}^{-1}$

$[\alpha]_{\mathrm{D}}{ }^{26}-10.9\left(c 0.65, \mathrm{CHCl}_{3}\right)$ 
$\mathbf{R}_{\mathbf{f}}(n$-Hexane: EtOAc $=3: 1$, color reagent: Hanessian's stain reagent): 0.45

The enantiomeric ratio was determined by HPLC using CHIRALPACK ${ }^{\circledR} \mathrm{IG}$ ( $n$-Hexane: $i$-PrOH $=66: 1$; flow rate $1.0 \mathrm{ml} / \mathrm{min}$, major isomer $t_{R}=11.4 \mathrm{~min}$, minor isomer $\left.t_{R}=20.4 \mathrm{~min}\right)(95 \% e e)$.

\section{Ethyl $(R, E)-5$-(2-bromophenyl)-7-oxo-9-(triisopropylsilyl)non-2-en-8-ynoate}

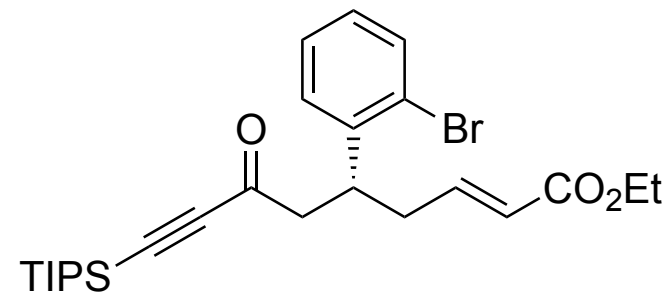

Yield: $70 \%(435.3 \mathrm{mg})$

Physical State: Yellow oil

${ }^{1} \mathrm{H}$ NMR $\left(\mathbf{C D C l}_{3}, 400 \mathrm{MHz}\right) \delta$ 1.05-1.17 (m, 21H), $1.26(\mathrm{t}, J=7.2 \mathrm{~Hz}, 3 \mathrm{H}), 2.52-2.64(\mathrm{~m}, 2 \mathrm{H}), 2.86(\mathrm{dd}, J=$ 8.0, $16.4 \mathrm{~Hz}, 1 \mathrm{H}), 2.92(\mathrm{dd}, J=6.8,16.0 \mathrm{~Hz}, 1 \mathrm{H}), 4.06$ (quin, $J=7.2 \mathrm{~Hz}, 1 \mathrm{H}), 4.14(\mathrm{q}, J=7.2 \mathrm{~Hz}, 2 \mathrm{H}), 5.78$ $(\mathrm{d}, J=15.6 \mathrm{~Hz}, 1 \mathrm{H}), 6.80(\mathrm{td}, J=7.2,15.6 \mathrm{~Hz}, 1 \mathrm{H}), 7.08(\mathrm{dt}, J=1.6,7.6 \mathrm{~Hz}, 1 \mathrm{H}), 7.17(\mathrm{dd}, J=1.6,7.6 \mathrm{~Hz}$, $1 \mathrm{H}), 7.27(\mathrm{t}, J=7.2 \mathrm{~Hz}, 1 \mathrm{H}), 7.55(\mathrm{dd}, J=0.80,8.0 \mathrm{~Hz}, 1 \mathrm{H})$

${ }^{13} \mathrm{C}$ NMR $\left(\mathrm{CDCl}_{3}, \mathbf{1 0 0} \mathrm{MHz}\right) \delta 185.1,166.1,145.0,140.9,133.4,128.4,127.8,127.8,124.8,123.8,103.9$, $96.7,60.2,49.8,39.0,37.0,18.5,18.5,18.5,18.5,18.5,18.5,14.2,11.0,11.0,11.0$

HRMS (ESI): [M+Na $]^{+}$calcd for $\mathrm{C}_{26} \mathrm{H}_{37} \mathrm{BrO}_{3} \mathrm{SiNa}$ : 527.1588, found: 527.1588

IR(neat) v 2146, 1721, 1676, 1470, 1367, 1267, 1205, 1162, 1107, 1024, 996 920, 883, 756, 680, 585, 452, $420 \mathrm{~cm}^{-1}$

$[\alpha]_{\mathrm{D}}^{26}-14.1\left(c 1.7, \mathrm{CHCl}_{3}\right)$

$\mathbf{R}_{\mathbf{f}}(n$-Hexane: EtOAc $=3: 1$, color reagent: Hanessian's stain reagent): 0.45

The enantiomeric ratio was determined by HPLC using CHIRALPACK ${ }^{\circledR}$ IG $(n$-Hexane: $i$-PrOH $=66: 1$; flow rate $1.0 \mathrm{ml} / \mathrm{min}$, major isomer $t_{R}=9.05 \mathrm{~min}$, minor isomer $\left.t_{R}=9.91 \mathrm{~min}\right)(95 \% e e)$.

\section{Ethyl $(R, E)-5$-(furan-2-yl)-7-oxo-9-(triisopropylsilyl)non-2-en-8-ynoate}

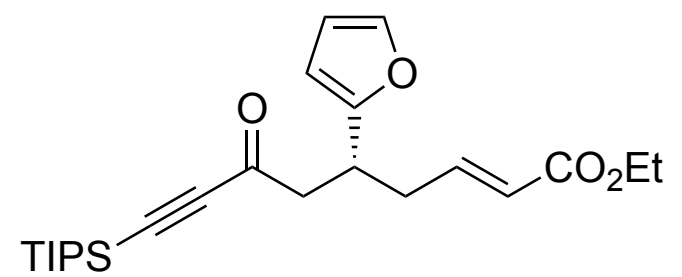

Yield: $65 \%(27.0 \mathrm{mg})$

Physical State: Yellow oil

${ }^{1} \mathbf{H}$ NMR $\left(\mathbf{C D C l}_{3}, 400 \mathrm{MHz}\right) \delta 1.04-1.17(\mathrm{~m}, 21 \mathrm{H}), 1.27(\mathrm{t}, J=7.2 \mathrm{~Hz}, 3 \mathrm{H}), 2.50-2.64(\mathrm{~m}, 2 \mathrm{H}), 2.82(\mathrm{dd}, J=$ $6.8,16.4 \mathrm{~Hz}, 1 \mathrm{H}), 2.94(\mathrm{dd}, J=7.2,16.4 \mathrm{~Hz}, 1 \mathrm{H}), 3.61$ (quin, $J=6.8 \mathrm{~Hz}, 1 \mathrm{H}$ ), 4.16 (q, $J=7.2 \mathrm{~Hz}, 2 \mathrm{H}$ ), 5.81 $(\mathrm{d}, J=15.6 \mathrm{~Hz}, 1 \mathrm{H}), 6.04(\mathrm{~d}, J=3.2 \mathrm{~Hz}, 1 \mathrm{H}), 6.26(\mathrm{dd}, J=2.0,3.2 \mathrm{~Hz}, 1 \mathrm{H}), 6.82(\mathrm{td}, J=7.6,15.6 \mathrm{~Hz}, 1 \mathrm{H})$, $7.31(\mathrm{~d}, J=1.6 \mathrm{~Hz}, 1 \mathrm{H})$ 
${ }^{13} \mathbf{C} \mathbf{N M R}\left(\mathbf{C D C l}_{3}, \mathbf{1 0 0} \mathrm{MHz}\right) \delta$ 185.0, 166.1, 155.2, 145.1, 141.6, 123.7, 110.1, 105.9, 103.9, 96.7, 60.3, 48.7, $36.0,33.9,18.5,18.5,18.5,18.5,18.5,18.5,14.2,11.0,11.0,11.0$

HRMS (ESI): [M+Na] $]^{+}$calcd for $\mathrm{C}_{24} \mathrm{H}_{36} \mathrm{O}_{4} \mathrm{SiNa}$ : 439.2275, found: 439.2272

IR(neat) $v 2146,1720,1681,1464,1367,1267,1161,1110,883,733,664 \mathrm{~cm}^{-1}$

$[\alpha]_{\mathrm{D}}{ }^{26}+1.04\left(c 0.75, \mathrm{CHCl}_{3}\right)$

$\mathbf{R}_{\mathbf{f}}(n$-Hexane: EtOAc $=3: 1$, color reagent: Hanessian's stain reagent): 0.20

The enantiomeric ratio was determined by HPLC using CHIRALPACK ${ }^{\circledR} \mathrm{IG}(n$-Hexane: $i$-PrOH $=66: 1$; flow rate $1.0 \mathrm{ml} / \mathrm{min}$, major isomer $t_{R}=11.9 \mathrm{~min}$, minor isomer $\left.t_{R}=16.5 \mathrm{~min}\right)(94 \% e e)$.

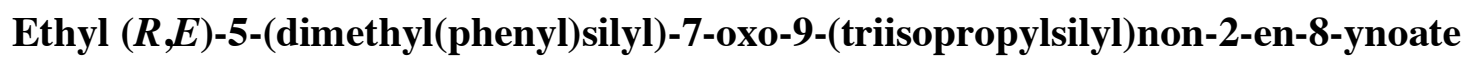

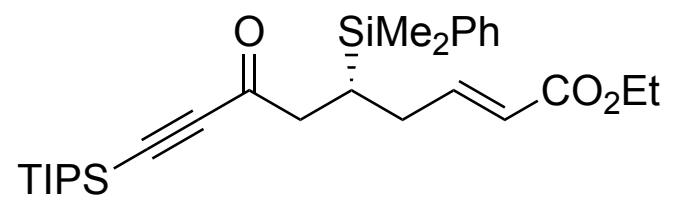

Yield: $85 \%(41.1 \mathrm{mg})$

Physical State: Yellow oil

${ }^{1} \mathrm{H}$ NMR $\left(\mathbf{C D C l}_{3}, 400 \mathrm{MHz}\right) \delta 0.32$ (s, 3H), 0.33 (s, 3H), 1.05-1.15 (m, 21H), 1.26 (t, J= 7.2 Hz, 3H), 1.79$1.86(\mathrm{~m}, 1 \mathrm{H}), 2.14$ (dquin, $J=1.2,8.0 \mathrm{~Hz}, 1 \mathrm{H}), 2.30-2.37(\mathrm{~m}, 1 \mathrm{H}), 2.47(\mathrm{dd}, J=8.4,16.4 \mathrm{~Hz}, 1 \mathrm{H}), 2.57(\mathrm{dd}$, $J=5.6,16.8 \mathrm{~Hz}, 1 \mathrm{H}), 4.14(\mathrm{q}, J=7.2 \mathrm{~Hz}, 2 \mathrm{H}), 5.72(\mathrm{~d}, J=15.6 \mathrm{~Hz}, 1 \mathrm{H}), 6.79(\mathrm{td}, J=7.2,15.6 \mathrm{~Hz}, 1 \mathrm{H}), 7.33$ $7.40(\mathrm{~m}, 3 \mathrm{H}), 7.46-7.50(\mathrm{~m}, 2 \mathrm{H})$

${ }^{13} \mathrm{C}$ NMR $\left(\mathrm{CDCl}_{3}, \mathbf{1 0 0} \mathrm{MHz}\right) \delta 187.5,166.1,147.8,136.7,136.7,133.9,129.4,128.0,122.7,122.7,104.1$, $96.1,60.1,45.7,32.6,21.1,18.5,18.5,18.5,18.5,18.5,18.5,14.2,11.0,11.0,11.0,-4.11,-4.11$

HRMS (ESI): $[\mathrm{M}+\mathrm{Na}]^{+}$calcd for $\mathrm{C}_{28} \mathrm{H}_{44} \mathrm{O}_{3} \mathrm{Si}_{2} \mathrm{Na}$ : 507.2721, found: 507.2723

IR(neat) $v 2145,1720,1676,1464,1428,1367,1261,1186,1112,1044,997,883,835,775,736,701,680$, $472 \mathrm{~cm}^{-1}$

$[\alpha]_{\mathrm{D}}{ }^{26}-6.45\left(c\right.$ 2.1, $\left.\mathrm{CHCl}_{3}\right)$

$\mathbf{R}_{\mathbf{f}}(n$-Hexane: EtOAc $=3: 1$, color reagent: Hanessian's stain reagent): 0.50

The enantiomeric ratio was determined by HPLC using CHIRALPACK ${ }^{\circledR} \mathrm{IG}$ ( $n$-Hexane: $i$-PrOH $=66: 1$; flow rate $1.0 \mathrm{ml} / \mathrm{min}$, major isomer $t_{R}=7.84 \mathrm{~min}$, minor isomer $\left.t_{R}=9.32 \mathrm{~min}\right)(98 \% e e)$.

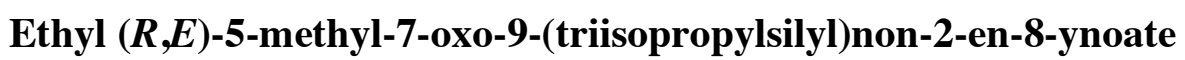

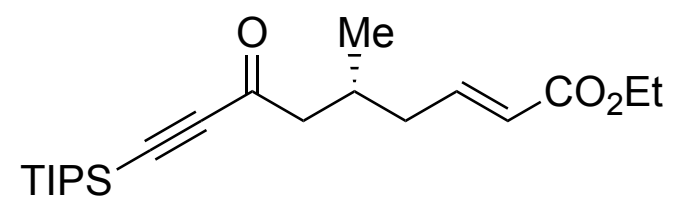

Yield: $60 \%(21.8 \mathrm{mg})$

Physical State: Yellow oil

${ }^{1} \mathrm{H}$ NMR $\left(\mathbf{C D C l}_{3}, \mathbf{4 0 0}\right.$ MHz) $\delta 0.99(\mathrm{~d}, J=6.8 \mathrm{~Hz}, 3 \mathrm{H}), 1.08-1.16(\mathrm{~m}, 21 \mathrm{H}), 1.29(\mathrm{t}, J=7.2 \mathrm{~Hz}, 3 \mathrm{H}), 2.10$ $1.17(\mathrm{~m}, 1 \mathrm{H}), 2.22-2.30(\mathrm{~m}, 1 \mathrm{H}), 2.30-2.39(\mathrm{~m}, 1 \mathrm{H}), 2.41(\mathrm{dd}, J=7.6,15.6 \mathrm{~Hz}, 1 \mathrm{H}), 2.57(\mathrm{dd}, J=6.0,15.6$ 
$\mathrm{Hz}, 1 \mathrm{H}), 4.19(\mathrm{q}, J=7.2 \mathrm{~Hz}, 2 \mathrm{H}), 5.84(\mathrm{dd}, J=1.2,15.6 \mathrm{~Hz}, 1 \mathrm{H}), 6.90(\mathrm{td}, J=7.2,15.6 \mathrm{~Hz}, 1 \mathrm{H})$

${ }^{13} \mathbf{C}$ NMR $\left(\mathbf{C D C l}_{3}, 100 \mathrm{MHz}\right) \delta 186.8,166.3,146.4,123.4,104.2,96.0,52.1,38.9,29.3,19.6,18.5,18.5$, $18.5,18.5,18.5,18.5,14.2,10.9,10.9,10.9,10.9$

HRMS (ESI): $[\mathrm{M}+\mathrm{Na}]^{+}$calcd for $\mathrm{C}_{21} \mathrm{H}_{36} \mathrm{O}_{3} \mathrm{SiNa}$ : 387.2326, found: 387.2325

IR(neat) $v 2146,1727,1677,1462,1070,882,682,522,483,459,431,421,413 \mathrm{~cm}^{-1}$

$[\alpha]_{\mathrm{D}}{ }^{26}+10.8\left(c 0.15, \mathrm{CHCl}_{3}\right)$

$\mathbf{R}_{\mathbf{f}}(n$-Hexane: EtOAc $=3: 1$, color reagent: Hanessian's stain reagent): 0.60

The enantiomeric ratio was determined by HPLC using CHIRALPACK ${ }^{\circledR}$ IG $(n$-Hexane: $i$-PrOH $=66: 1$; flow rate $1.0 \mathrm{ml} / \mathrm{min}$, major isomer $t_{R}=5.91 \mathrm{~min}$, minor isomer $\left.t_{R}=6.39 \mathrm{~min}\right)(76 \% e e)$.

\subsection{One-pot procedure of providing compound 10 from compound 1 and compound $2 \mathrm{~g}$}

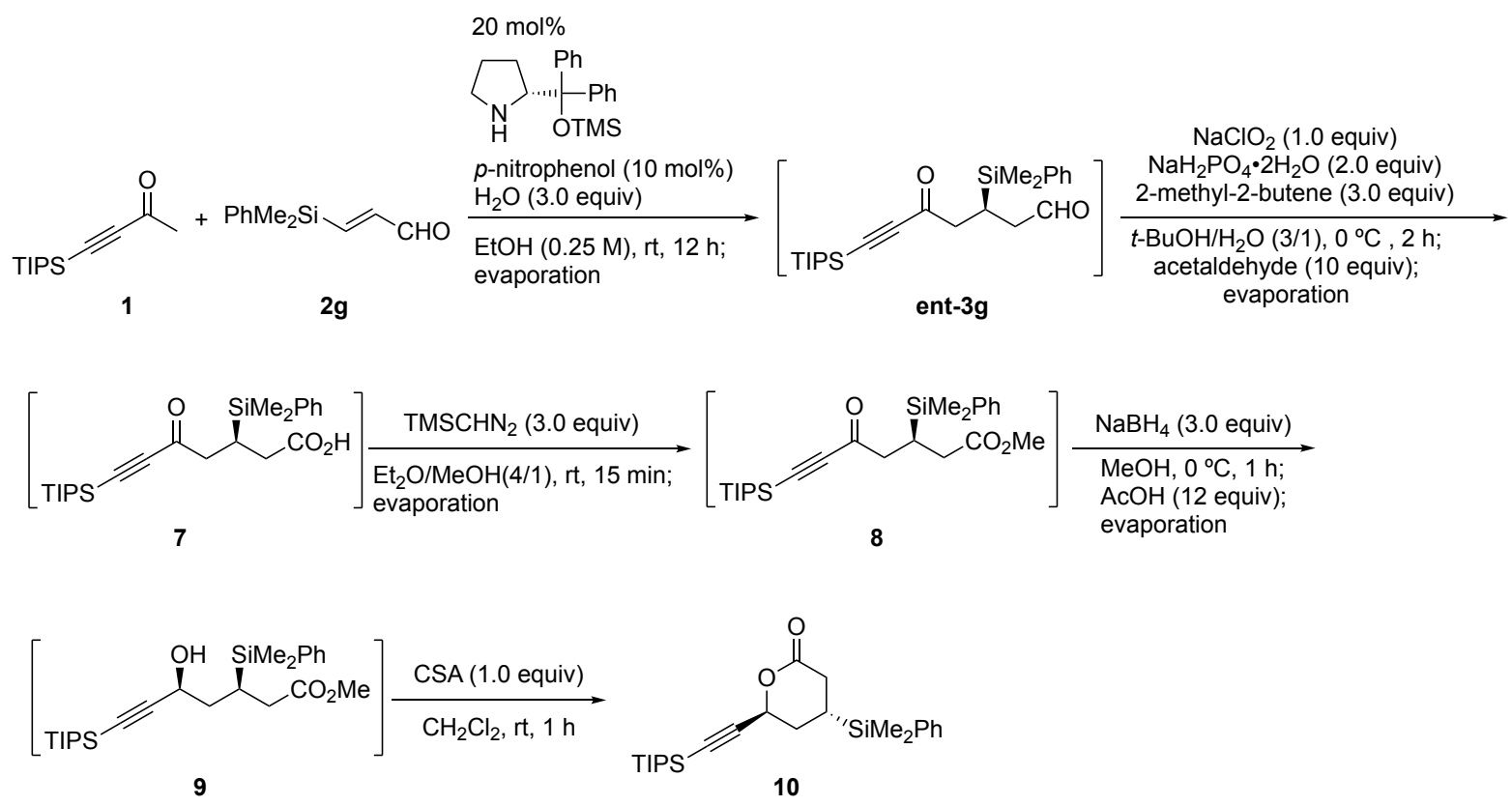

To a solution of aldehyde $\mathbf{2 g}$ (114 mg, $0.60 \mathrm{mmol}$ ) and 4-(triisopropylsilyl)but-3-yn-2-one 1 (112 mg, 0.50 mmol) in EtOH $(2.0 \mathrm{~mL}), \mathrm{H}_{2} \mathrm{O}(27 \mu \mathrm{L}, 1.5 \mathrm{mmol})$, diphenylprolinol silyl ether (32.5 $\left.\mathrm{mg}, 0.10 \mathrm{mmol}\right)$ and $p$ nitrophenol $(7.0 \mathrm{mg}, 0.050 \mathrm{mmol})$ were added at room temperature. After stirred at this temperature for 12 hours, its solvent was removed by evaporation under reduced pressure. To a solution of the residue in $t$ $\mathrm{BuOH} / \mathrm{H}_{2} \mathrm{O}=(3 / 1)(0.8 \mathrm{~mL}), \mathrm{NaH}_{2} \mathrm{PO}_{4} \cdot \mathrm{H}_{2} \mathrm{O}(312 \mathrm{mg}, 1.0 \mathrm{mmol}), 2$-methyl-2-butene $(160 \mu \mathrm{L}, 1.5 \mathrm{mmol})$ and $\mathrm{NaClO}_{2}(45 \mathrm{mg}, 0.5 \mathrm{mmol})$ were added at $0{ }^{\circ} \mathrm{C}$. After stirred at this temperature for 2 hours, the oxidant in the reaction mixture was quenched by acetaldehyde $(0.14 \mathrm{~mL}, 2.5 \mathrm{mmol})$. Its solvent and excess amount of acetaldehyde were removed by evaporation under reduced pressured. To a solution of the residue in $\mathrm{Et}_{2} \mathrm{O}$ : $\mathrm{MeOH}=(4: 1)(1.0 \mathrm{~mL}), \mathrm{TMSCHN}_{2}\left(2.0 \mathrm{M}\right.$ in $\left.\mathrm{Et}_{2} \mathrm{O}, 75 \mu \mathrm{L}, 1.5 \mathrm{mmol}\right)$ was added at room temperature. After stirred at this temperature for 15 minutes, its solvent was removed by evaporation under reduced pressure. To a solution of the residue in $\mathrm{MeOH}(1.0 \mathrm{~mL})$, sodium borohydride $(56.0 \mathrm{mg}, 1.5 \mathrm{mmol})$ was added at $0{ }^{\circ} \mathrm{C}$. After stirred at this temperature for 1 hour, the reductant was quenched by acetic acid $(0.24 \mathrm{~mL}, 6.0 \mathrm{mmol})$. Its solvent and acetic acid were removed by evaporation under reduced pressure. To a solution of the residue 
in $\mathrm{CH}_{2} \mathrm{Cl}_{2}(1.5 \mathrm{~mL}), \mathrm{CSA}(117 \mathrm{mg}, 0.5 \mathrm{mmol})$ was added at room temperature. After stirred at this temperature for 1 hour, the reaction mixture was quenched by sat. aq. $\mathrm{NaHCO}_{3}(5 \mathrm{~mL})$ and diluted by EtOAc $(5 \mathrm{~mL})$. After separated, the aqueous layer was extracted with EtOAc $(5 \mathrm{~mL})$ for three times. The combined organic layers were washed by brine $(10 \mathrm{~mL})$, dried over on sodium sulfate, and evaporated under reduced pressure. The crude material was purified by column chromatography on silica gel $(n$-Hexane: EtOAc $=10: 1)$ to give desired compound 10 ( $85.0 \mathrm{mg}, 0.21 \mathrm{mmol})$ in $42 \%$ isolated yield.

(4R,6S)-4-(Dimethyl(phenyl)silyl)-6-((triisopropylsilyl)ethynyl)tetrahydro-2H-pyran-2-one (10)<smiles>CS[C@H]1CC(=O)O[C@H](C#CSP)C1</smiles>

Yield: $42 \%(85.0 \mathrm{mg})$

Physical State: Colorless oil

${ }^{1} \mathrm{H}$ NMR $\left(\mathbf{C D C l}_{3}, 400 \mathrm{MHz}\right) \delta 0.33$ (s, 3H), 0.33 (s, 3H), 1.02-1.11 (m, 21H), 1.78-2.00 (m, 3H), 2.27 (dd, $J$ $=12.0,18.0 \mathrm{~Hz}, 1 \mathrm{H}), 2.62(\mathrm{ddd}, J=1.6,6.0,18.0 \mathrm{~Hz}, 1 \mathrm{H}), 5.19(\mathrm{dd}, J=3.2,4.4 \mathrm{~Hz}, 1 \mathrm{H}), 7.35-7.42(\mathrm{~m}, 3 \mathrm{H})$, 7.45-7.48 (m, 2H)

${ }^{13} \mathbf{C ~ N M R}\left(\mathbf{C D C l}_{3}, \mathbf{1 0 0} \mathbf{M H z}\right) \delta 170.1,135.3,133.8,133.8,129.7,128.1,128.1,103.7,89.1,70.4,30.7,29.8$, $18.5,18.5,18.5,18.5,18.5,18.5,15.6,11.0,11.0,11.0,-5.65,-5.76$

HRMS (ESI): [M+Na] ${ }^{+}$calcd for $\mathrm{C}_{24} \mathrm{H}_{38} \mathrm{O}_{2} \mathrm{Si}_{2} \mathrm{Na}$ : 437.2303, found: 437.2300

IR(neat) $v 2943,2865,2317,1746,1464,1261,1065,977,883,814,702,668 \mathrm{~cm}^{-1}$

$[\alpha]_{\mathrm{D}}{ }^{26}+43.9\left(c 1.5, \mathrm{CHCl}_{3}\right)$

$\mathbf{R}_{\mathbf{f}}(n$-Hexane: EtOAc $=2: 1$, color reagent: Hanessian's stain reagent $): 0.45$

\subsection{One-pot procedure of providing compound 6 from compound 10}
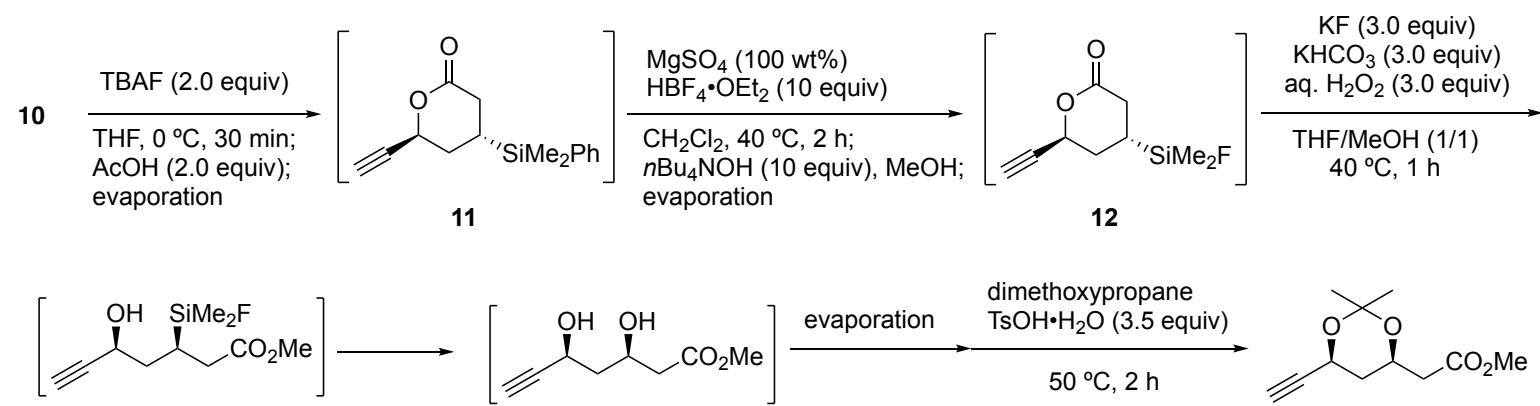

13

14

6

To a solution of compound $\mathbf{1 0}(82.8 \mathrm{mg}, 0.20 \mathrm{mmol})$ in THF, TBAF (1.0 M in THF, $300 \mu \mathrm{L}, 0.30 \mathrm{mmol})$ was added at $0{ }^{\circ} \mathrm{C}$. After stirred at this temperature for 30 minutes, acetic acid $(34.3 \mu \mathrm{L}, 0.60 \mathrm{mmol})$ was added. Then solvent was removed by evaporation under reduced pressure. To a solution of the residue in 
$\mathrm{CH}_{2} \mathrm{Cl}_{2}(0.30 \mathrm{~mL}), \mathrm{MgSO}_{4}(82.8 \mathrm{mg})$ and $\mathrm{HBF}_{4} \cdot \mathrm{OEt}_{2}(272 \mu \mathrm{L}, 2.0 \mathrm{mmol})$ were added at $40{ }^{\circ} \mathrm{C}$. After stirred at this temperature for 2 hours, TBAOH (10\% in $\mathrm{MeOH}, 5.2 \mathrm{~mL}, 2.0 \mathrm{mmol})$ was added. Then solvent was removed by evaporation under reduced pressure. To a solution of the residue in THF: $\mathrm{MeOH}=(1: 1)(0.30$ $\mathrm{mL}), \mathrm{KF}(34.9 \mathrm{mg}, 0.60 \mathrm{mmol}), \mathrm{KHCO}_{3}(60.0 \mathrm{mg}, 0.60 \mathrm{mmol})$ and aq. $\mathrm{H}_{2} \mathrm{O}_{2}\left(35 \%\right.$ in $\mathrm{H}_{2} \mathrm{O}, 40.2 \mu \mathrm{L}, 0.60$ $\mathrm{mmol}$ ) were added at $40{ }^{\circ} \mathrm{C}$. After stirred at this temperature for 2 hours, solvent was removed by evaporation under reduced pressure. To a solution of the residue in 2,2-dimethoxy propane $(0.60 \mathrm{~mL}), \mathrm{TsOH} \cdot \mathrm{H}_{2} \mathrm{O}(133$ $\mathrm{mg}, 0.70 \mathrm{mmol}$ ) was added at $50{ }^{\circ} \mathrm{C}$. After stirred at this temperature for 2 hours, the reaction mixture was quenched by sat. aq. $\mathrm{NaHCO}_{3}(5 \mathrm{~mL})$ and diluted by EtOAc $(5 \mathrm{~mL})$. The aqueous layer was extracted with EtOAc $(5 \mathrm{~mL})$ for three times. The combined organic layers were washed with brine $(20 \mathrm{~mL})$, dried over on sodium sulfate. Its solvent was removed by evaporation under reduced pressure. The residue was purified by column chromatography on silica gel $(n$-Hexane: EtOAc $=4: 1)$ to give corresponding compound $\mathbf{6}(17.0 \mathrm{mg}$, $0.080 \mathrm{mmol}$ ) in $40 \%$ isolated yield.

Methyl 2-((4R,6S)-6-ethynyl-2,2-dimethyl-1,3-dioxan-4-yl)acetate (6)

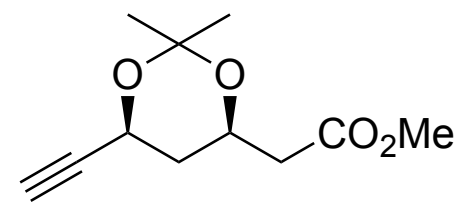

Yield: $40 \%(17.0 \mathrm{mg})$

Physical State: Colorless oil

${ }^{1} \mathrm{H}$ NMR $\left(\mathbf{C D C l}_{3}, 400 \mathrm{MHz}\right) \delta 1.42(\mathrm{~s}, 3 \mathrm{H}), 1.47(\mathrm{~s}, 3 \mathrm{H}), 1.67(\mathrm{t}, J=13.2 \mathrm{~Hz}, 1 \mathrm{H}), 1.85$ (td, $J=2.4,16.8 \mathrm{~Hz}$, $1 \mathrm{H}), 2.40(\mathrm{dd}, J=6.0,16.0 \mathrm{~Hz}, 1 \mathrm{H}), 2.45(\mathrm{~d}, J=2.0 \mathrm{~Hz}, 1 \mathrm{H}), 2.56(\mathrm{dd}, J=7.2,16.0 \mathrm{~Hz}, 1 \mathrm{H}), 3.69(\mathrm{~s}, 3 \mathrm{H})$, $4.31(\mathrm{dsext}, J=2.4,6.4 \mathrm{~Hz}, 1 \mathrm{H}), 4.69(\mathrm{td}, J=2.4,11.6 \mathrm{~Hz}, 1 \mathrm{H})$

${ }^{13} \mathbf{C}$ NMR $\left(\mathbf{C D C l}_{3}, \mathbf{1 0 0} \mathbf{M H z}\right) \delta 171.0,99.6,82.2,72.9,65.4,60.0,51.7,40.8,36.7,30.0,19.3$

HRMS (ESI): [M+Na] $]^{+}$calcd for $\mathrm{C}_{11} \mathrm{H}_{16} \mathrm{O}_{4} \mathrm{Na}$ : 235.0941, found: 235.0940

IR(neat) $v 3276,2926,2126,1728,1462,1381,1262,1200,1166,1123,1011,924,845,743,665,557 \mathrm{~cm}^{-1}$ $[\alpha]_{\mathrm{D}}{ }^{26}-1.97\left(c 1.1, \mathrm{CHCl}_{3}\right)$

$\mathbf{R}_{\mathbf{f}}(n$-Hexane: EtOAc $=2: 1$, color reagent: Hanessian's stain reagent $): 0.40$

\subsection{Lindlar reduction of compound 6 to determine its absolute configuration}

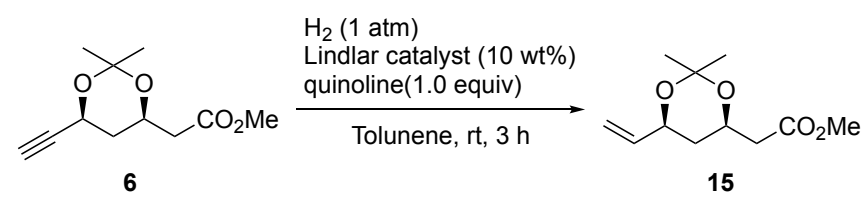

To a solution of compound $7(12.7 \mathrm{mg}, 0.060 \mathrm{mmol})$ in toluene $(1.0 \mathrm{~mL})$, Lindlar's catalyst $(2.0 \mathrm{mg})$ and quinolone $(7.11 \mu \mathrm{L}, 0.060 \mathrm{mmol})$ were added at room temperature under $\mathrm{H}_{2}$ atmosphere $(1 \mathrm{~atm})$. After stirred at this condition for 3 hours, the residue was filtrated by Celite ${ }^{\circledR}$. The solvent was removed by evaporation under reduced pressure. The residue was purified by column chromatography on silica gel ( $n$-Hexane: EtOAc 
$=4: 1)$ to give corresponding compound $15(11.7 \mathrm{mg}, 0.055 \mathrm{mmol})$ in $91 \%$ isolated yield. All Spectrum data of target compound were matched to reported data ${ }^{1)}$. The reported value of optical rotation is $[\alpha]_{\mathrm{D}}^{20}-2.8(c 1.4$, $\left.\mathrm{CHCl}_{3}\right)$ and observed one was $[\alpha]_{\mathrm{D}}{ }^{27}-2.9\left(c 1.0 \mathrm{CHCl}_{3}\right)$. Thus, absolute configuration of compound $\mathbf{1 5}$ is same to reported one.

Methyl 2-((4R,6S)-2,2-dimethyl-6-vinyl-1,3-dioxan-4-yl)acetate (15)<smiles>C=C[C@H]1C[C@@H](CC(C)=O)OC(C)(C)O1</smiles>

Yield: $91 \%(11.7 \mathrm{mg})$

Physical State: Colorless oil

${ }^{1} \mathrm{H}$ NMR $\left(\mathbf{C D C l}_{3}, 400 \mathrm{MHz}\right) \delta 1.30(\mathrm{q}, J=12.8,1 \mathrm{H}), 1.41(\mathrm{~s}, 3 \mathrm{H}), 1.49(\mathrm{~s}, 3 \mathrm{H}), 1.66(\mathrm{td}, J=2.4,12.8 \mathrm{~Hz}$, $1 \mathrm{H}), 2.40(\mathrm{dd}, J=6.4,15.6 \mathrm{~Hz}, 1 \mathrm{H}), 2.57(\mathrm{dd}, J=6.8,15.6 \mathrm{~Hz}, 1 \mathrm{H}), 3.69(\mathrm{~s}, 3 \mathrm{H}), 4.32-4.41(\mathrm{~m}, 2 \mathrm{H}), 5.13(\mathrm{td}$, $J=1.6,10.4 \mathrm{~Hz}, 1 \mathrm{H}), 5.26(\mathrm{td}, J=1.2,17.2 \mathrm{~Hz}, 1 \mathrm{H}), 5.81(\mathrm{ddd}, J=6.0,10.4,17.2 \mathrm{~Hz}, 1 \mathrm{H})$

${ }^{13} \mathbf{C} \mathbf{N M R}\left(\mathbf{C D C l}_{3}, \mathbf{1 0 0} \mathbf{M H z}\right) \delta 171.3,138.4,115.6,98.9,70.0,65.6,51.7,41.1,36.2,30.0,19.7$

HRMS (ESI): $[\mathrm{M}+\mathrm{Na}]^{+}$calcd for $\mathrm{C}_{11} \mathrm{H}_{18} \mathrm{O}_{4} \mathrm{Na}: 237.1097$, found: 237.1097

IR(neat) $v$ 2994, 2951, 1740, 1438, 1381, 1314, 1259, 1200, 1169, 1097, 999, 925, $848 \mathrm{~cm}^{-1}$

$[\alpha]_{\mathrm{D}}^{27}-2.9\left(c 1.0, \mathrm{CHCl}_{3}\right)$

$\mathbf{R}_{\mathbf{f}}(n$-Hexane: EtOAc $=2: 1$, color reagent: Hanessian's stain reagent $): 0.35$

\subsection{Preparation of compound 8}

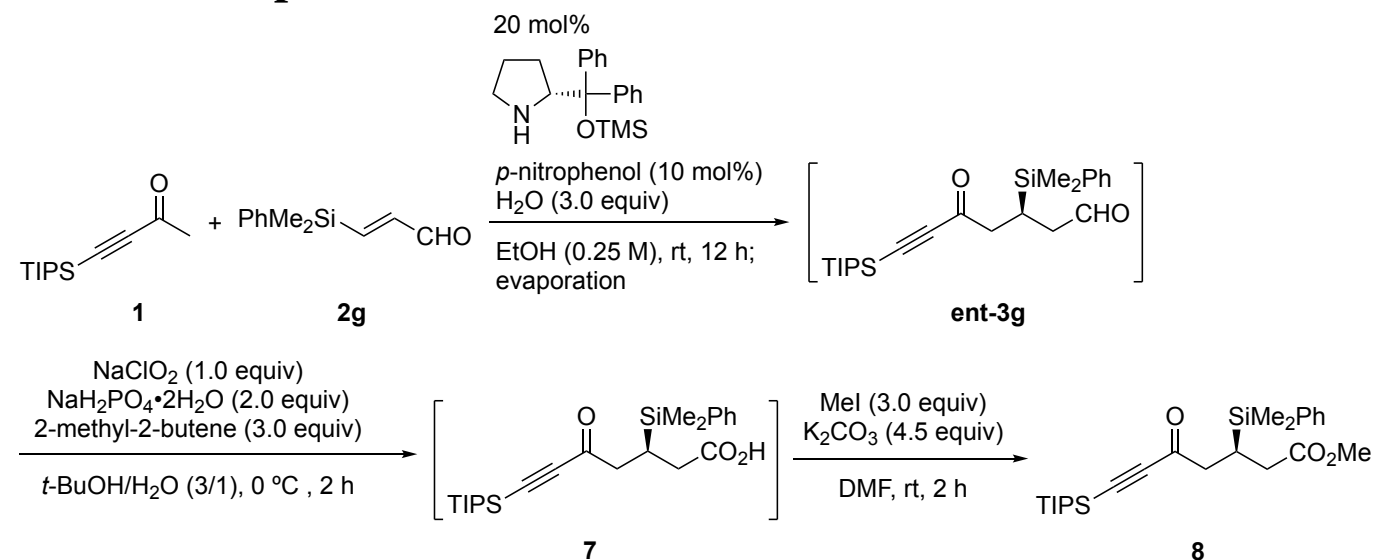

To a solution of aldehyde $\mathbf{2 g}$ (1.33 g, $7.0 \mathrm{mmol})$ and 4-(triisopropylsilyl)but-3-yn-2-one $\mathbf{1}$ (1.57 g, $7.0 \mathrm{mmol})$ in $\mathrm{EtOH}(21 \mathrm{~mL}), \mathrm{H}_{2} \mathrm{O}(378 \mu \mathrm{L}, 21 \mathrm{mmol})$, diphenylprolinol silyl ether (456 mg, $\left.1.4 \mathrm{mmol}\right)$ and $p$-nitrophenol $(97.4 \mathrm{mg}, 0.70 \mathrm{mmol})$ were added at room temperature. After stirred the reaction mixture at this temperature for 3 hours, its solvent was removed under reduced pressure. To a solution of the residue in $t-\mathrm{BuOH}: \mathrm{H}_{2} \mathrm{O}=$ (3:1) (21 mL), $\mathrm{NaH}_{2} \mathrm{PO}_{4} \cdot \mathrm{H}_{2} \mathrm{O}(2.18 \mathrm{~g}, 14 \mathrm{mmol})$, 2-methyl-2-butene (2.23 mL, $\left.21 \mathrm{mmol}\right)$ and $\mathrm{NaClO}_{2}(633$ $\mathrm{mg}, 7.0 \mathrm{mmol}$ ) were added at $0{ }^{\circ} \mathrm{C}$. After stirred at this temperature for 2 hours, the reaction mixture was quenched by sat. aq. sodium hyposulfite $(50 \mathrm{~mL})$. The residue was diluted by EtOAc $(20 \mathrm{~mL})$. After separated, 
the aqueous layer was extracted with EtOAc $(30 \mathrm{~mL})$ for three times. The combined organic layers were evaporated under reduced pressure. The residue was directly used without further purification. To a solution of crude material in DMF (30 mL), MeI $(2.18 \mathrm{~mL}, 35 \mathrm{mmol})$ and $\mathrm{K}_{2} \mathrm{CO}_{3}(7.26 \mathrm{~g}, 75 \mathrm{mmol})$ were added at room temperature. After stirred at this temperature for 2 hours, the reaction mixture was quenched by sat. aq. ammonium chloride $(60 \mathrm{~mL})$. The residue was diluted by EtOAc $(30 \mathrm{~mL})$. After separated, the aqueous layer was extracted with EtOAc $(50 \mathrm{~mL})$ for three times. The combined organic layers were washed by brine, dried over on sodium sulfate, and evaporated under reduced pressure. The crude material was purified by column chromatography on silica gel $(n$-Hexane: EtOAc $=15: 1)$ to give the target compound $\mathbf{8}(1.71 \mathrm{~g}, 3.85 \mathrm{mmol})$ in $55 \%$ yield.

Methyl (R)-3-(dimethyl(phenyl)silyl)-5-oxo-7-(triisopropylsilyl)hept-6-ynoate (8)

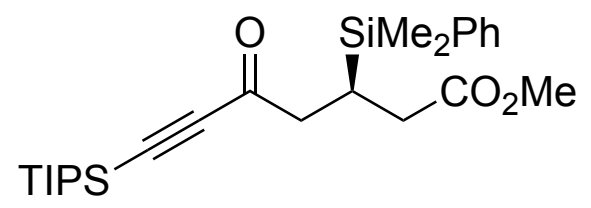

Yield: $55 \%(1.71 \mathrm{~g})$

Physical State: Brown oil

${ }^{1}$ H NMR $\left(\mathbf{C D C l}_{3}, 400 \mathrm{MHz}\right) \delta 0.33(\mathrm{~s}, 6 \mathrm{H}), 1.07-1.15(\mathrm{~m}, 21 \mathrm{H}), 2.05-2.12(\mathrm{~m}, 1 \mathrm{H}), 2.26(\mathrm{dd}, J=8.0,15.6$ $\mathrm{Hz}, 1 \mathrm{H}), 2.41(\mathrm{dd}, J=6.0,15.6 \mathrm{~Hz}, 1 \mathrm{H}), 2.55(\mathrm{dd}, J=8.8,16.8 \mathrm{~Hz}, 1 \mathrm{H}), 2.66(\mathrm{dd}, J=8.8,16.8 \mathrm{~Hz}, 1 \mathrm{H}) 3.56$ (s, 3H), 7.33-7.39 (m, 3H), 7.48-7.50 (m, 2H)

${ }^{13} \mathbf{C}$ NMR (CDCl 3 , 100 MHz) $\delta$ 187.2, 173.3, 136.4, 133.9, 133.9, 129.4, 127.9, 127.9, 104.0, 95.7, 51.4, 46.0, $34.4,32.8,18.5,18.5,18.5,18.5,18.5,18.5,11.0,11.0,11.0,-4.48,-4.52$

HRMS (ESI): [M+Na] $]^{+}$calcd for $\mathrm{C}_{25} \mathrm{H}_{40} \mathrm{O}_{3} \mathrm{Si}_{2} \mathrm{Na}$ : 467.2408, found: 467.2411

IR(neat) $v 2946,2867,2146,1721,1676,1464,1428,1367,1261,1185,1112,1044,997,883,835,815,775$, $736,701,680,581 \mathrm{~cm}^{-1}$

$[\alpha]_{\mathrm{D}}{ }^{26}+14.3\left(c 0.90, \mathrm{CHCl}_{3}\right)$

$\mathbf{R}_{\mathbf{f}}(n$-Hexane: EtOAc $=3: 1$, color reagent: Hanessian's stain reagent $): 0.55$

\subsection{Stereo-selective reduction of compound 8}

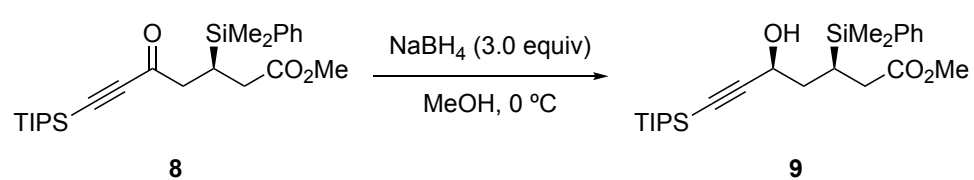

To a solution of compound 8 (44.5 mg, $0.10 \mathrm{mmol})$ in $\mathrm{MeOH}(300 \mu \mathrm{L})$, sodium borohydride (11.3 mg, 0.30 mmol) was added at $0{ }^{\circ} \mathrm{C}$. After stirred at this temperature for 1 hour, the reaction mixture was quenched by sat. aq. ammonium chloride $(5 \mathrm{~mL})$. The residue was diluted by EtOAc $(5 \mathrm{~mL})$. After separated, the aqueous layer was extracted with EtOAc $(5 \mathrm{~mL})$ for three times. The combined organic layers were washed by brine $(20 \mathrm{~mL})$ and dried over on sodium sulfate. The solvent was removed by evaporation under reduced pressure. 
The residue was purified by column chromatography on silica gel $(n$-Hexane: EtOAc $=8: 1)$ to give the target compound 9 (39.2 $\mathrm{mg}, 0.088 \mathrm{mmol}$ ) in $88 \%$ yield.

Methyl (3R,5S)-3-(dimethyl(phenyl)silyl)-5-hydroxy-7-(triisopropylsilyl)hept-6-ynoate (9)

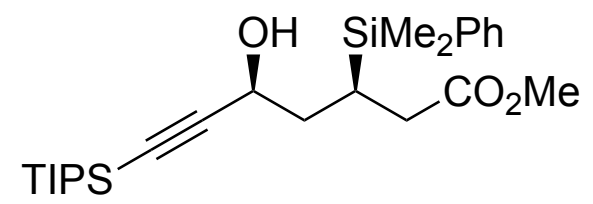

Yield: $88 \%(39.2 \mathrm{mg})$

Physical State: Brown oil

${ }^{1} \mathrm{H}$ NMR $\left(\mathrm{CDCl}_{3}, 400 \mathrm{MHz}\right) \delta 0.321(\mathrm{~s}, 6 \mathrm{H}), 1.03-1.14(\mathrm{~m}, 21 \mathrm{H}), 1.60-1.66(\mathrm{~m}, 1 \mathrm{H}), 1.70-1.81(\mathrm{~m}, 1 \mathrm{H})$, 1.87-1.95 (m, 1H), $2.39(\mathrm{dd}, J=8.0,16.4 \mathrm{~Hz}, 1 \mathrm{H}), 2.46(\mathrm{dd}, J=8.0,16.4 \mathrm{~Hz}, 1 \mathrm{H}), 3.60(\mathrm{~d}, J=0.80 \mathrm{~Hz}, 3 \mathrm{H})$, $4.31(\mathrm{dd}, J=4.0,8.8 \mathrm{~Hz}, 1 \mathrm{H}), 7.34-7.36(\mathrm{~m}, 3 \mathrm{H}), 7.48-7.50(\mathrm{~m}, 2 \mathrm{H})$

${ }^{13} \mathbf{C ~ N M R}\left(\mathbf{C D C l}_{3}, \mathbf{1 0 0} \mathrm{MHz}\right) \delta 175.5,137.0,133.9,133.9,129.3,127.9,127.9,108.6,85.4,61.2,51.9,39.1$, $34.2,18.6,18.6,18.6,18.6,18.6,18.6,11.1,11.1,11.1,-4.32,-4.87$

HRMS (ESI): $[\mathrm{M}+\mathrm{Na}]^{+}$calcd for $\mathrm{C}_{25} \mathrm{H}_{42} \mathrm{O}_{3} \mathrm{Si}_{2} \mathrm{Na}$ : 469.2565 , found: 469.2564

IR(neat)v 2944, 2866, 2169, 1739, 1463, 1252, 1113, 1017, 883, 834, 816, 774, 735, 701, $678 \mathrm{~cm}^{-1}$

$[\alpha]_{\mathrm{D}}{ }^{26}-3.04\left(c\right.$ 2.5, $\left.\mathrm{CHCl}_{3}\right)$

$\mathbf{R}_{\mathbf{f}}(n$-Hexane: EtOAc $=2: 1$, color reagent: Hanessian's stain reagent $): 0.45$

\subsection{Deprotection of compound 9}

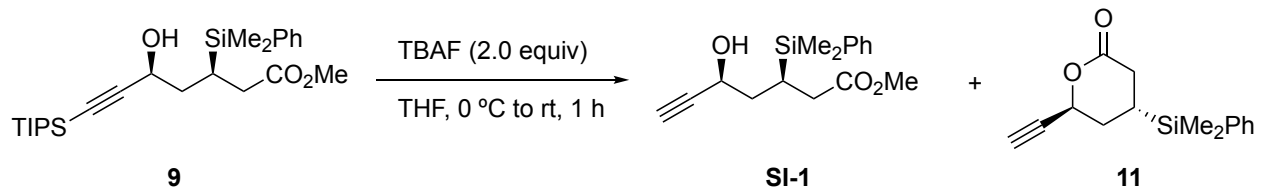

To a solution of compound $9(133 \mathrm{mg}, 0.30 \mathrm{mmol})$ in THF $(0.90 \mathrm{~mL})$, TBAF $(1.0 \mathrm{M}$ in THF, $0.60 \mathrm{~mL}, 0.60$ mmol) was added at $0{ }^{\circ} \mathrm{C}$ with stirring. Then, the reaction temperature was elevated to room temperature. After stirred at this temperature for 1 hour, the reaction mixture was quenched by sat. aq. ammonium chloride $(20 \mathrm{~mL})$. The residue was diluted by EtOAc $(10 \mathrm{~mL})$. After separated, aqueous layer was extracted with EtOAc $(10 \mathrm{~mL})$ for three times. The combined organic layers were dried over on sodium sulfate and evaporated under reduced pressure. The residue was purified by column chromatography on silica gel ( $n$-Hecane/EtOAc $=$ 1/6 1/2) to give desired alcohol SI-1 (23.5 mg, 27\%) and undesired lactone 11 (47.2 mg, 61\%).

\section{Methyl (3R,5S)-3-(dimethyl(phenyl)silyl)-5-hydroxyhept-6-ynoate (SI-1)}<smiles>C#C[C@@H](O)C[C@@H](CC(C)=O)c1ccccc1</smiles>

Yield: $27 \%(23.5 \mathrm{mg})$

Physical State: Colorless oil 
${ }^{1} \mathrm{H}$ NMR $\left(\mathbf{C D C l}_{3}, 400 \mathrm{MHz}\right) \delta 0.322(\mathrm{~s}, 6 \mathrm{H}), 1.57-1.64(\mathrm{~m}, 1 \mathrm{H}), 1.67-1.73(\mathrm{~m}, 1 \mathrm{H}), 1.94$ (ddd, $J=2.8,9.6$, $12.8 \mathrm{~Hz}, 1 \mathrm{H}), 2.22(\mathrm{dd}, J=8.4,16.8 \mathrm{~Hz}, 1 \mathrm{H}), 2.42-2.43(\mathrm{~m}, 1 \mathrm{H}), 2.47(\mathrm{dd}, J=3.6,17.2 \mathrm{~Hz}, 1 \mathrm{H}), 3.63(\mathrm{~s}, 3 \mathrm{H})$, $4.27(\mathrm{~d}, J=9.6 \mathrm{~Hz}, 1 \mathrm{H}), 7.35-7.37(\mathrm{~m}, 3 \mathrm{H}), 7.49-7.51(\mathrm{~m}, 2 \mathrm{H})$

${ }^{13} \mathbf{C} \mathbf{N M R}\left(\mathbf{C D C l}_{3}, \mathbf{1 0 0} \mathrm{MHz}\right) \delta 175.9,136.7,133.9,133.9,129.3,127.9,127.9,84.8,72.7,60.3,52.0,38.9$, $34.0,16.8,-4.47,-4.92$

HRMS (ESI): $[\mathrm{M}+\mathrm{Na}]^{+}$calcd for $\mathrm{C}_{16} \mathrm{H}_{22} \mathrm{O}_{3}$ SiNa: 313.1230 , found: 313.1235

$\operatorname{IR}($ neat $) v 3448,3291,3070,2952,2116,1733,1428,1253,1113,1043,816,775,736,702 \mathrm{~cm}^{-1}$

$[\alpha]_{\mathrm{D}}{ }^{26}-18.2\left(c 0.50, \mathrm{CHCl}_{3}\right)$

$\mathbf{R}_{\mathbf{f}}(n$-Hexane: EtOAc $=2: 1$, color reagent: Hanessian's stain reagent $): 0.25$

(4R,6S)-4-(Dimethyl(phenyl)silyl)-6-ethynyltetrahydro-2H-pyran-2-one (11)<smiles>C#C[C@H]1C[C@H](Sc2ccccc2)CC(=O)O1</smiles>

Yield: $61 \%(47.2 \mathrm{mg})$

Physical State: Yellow oil

${ }^{1} \mathrm{H}$ NMR $\left(\mathbf{C D C l}_{3}, 400\right.$ MHz) 0.343 (s, 3H), 0.347 (s, 3H), 1.83-1.96 (m, 3H), 2.24-2.36 (s, 1H), 2.58 (dd, $J=$ 2.0, $10.0 \mathrm{~Hz}, 1 \mathrm{H}), 2.64(\mathrm{dd}, J=4.2,16.4 \mathrm{~Hz}, 1 \mathrm{H}), 5.14$ (quin, $J=2.0 \mathrm{~Hz}, 1 \mathrm{H}), 7.36-7.42$ (m, 2H), 7.46-7.50 $(\mathrm{m}, 3 \mathrm{H})$

${ }^{13} \mathbf{C}$ NMR (CDCl 3 , 100 MHz) $\delta 170.0,135.2,133.8,133.8,129.8,128.1,128.1,80.2,75.6,69.4,30.6,29.2$, $15.3,-5.60,-5.69$

HRMS (ESI): [M+Na] $]^{+}$calcd for $\mathrm{C}_{15} \mathrm{H}_{18} \mathrm{O}_{2} \mathrm{SiNa}$ : 281.0968, found: 281.0964

IR(neat) $v 3265,2954,2092,1735,1683,1428,1253,1113,816,776,737,702 \mathrm{~cm}^{-1}$

$[\alpha]_{\mathrm{D}}{ }^{26}+53.9\left(c 1.0, \mathrm{CHCl}_{3}\right)$

$\mathbf{R}_{\mathbf{f}}(n$-Hexane: EtOAc $=2: 1$, color reagent: Hanessian's stain reagent $): 0.30$

\subsection{Oxidation of compound SI-1}

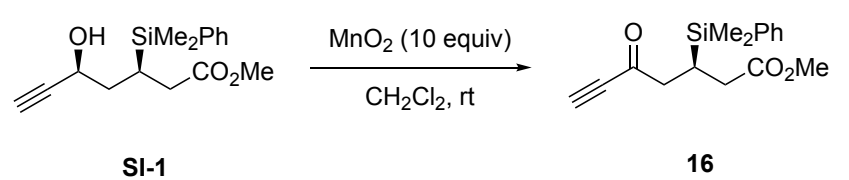

To a solution of alcohol SI-1 (20 mg, $0.076 \mathrm{mmol})$ in $\mathrm{CH}_{2} \mathrm{Cl}_{2}(1.0 \mathrm{~mL}), \mathrm{Mn}_{2} \mathrm{O}$ (65.4 mg, $\left.0.76 \mathrm{mmol}\right)$ was added at room temperature. After stirred at this temperature for 1 hour, its solvent was removed under reduced pressure. The residue was diluted by EtOAc and filtrated by silica gel. After removed its solvent under reduced pressure, the reaction mixture was purified by column chromatography on silica gel $(n$-Hexane: EtOAc $=3: 1)$ to give desired ketone 16 (16.9 $\mathrm{mg}, 0.058 \mathrm{mmol})$ in $76 \%$. 
<smiles>C#CC(=O)C[C@H](CC(C)=O)c1ccccc1</smiles>

Yield: $76 \%(16.9 \mathrm{mg})$

Physical State: Colorless oil

${ }^{1} \mathrm{H}$ NMR $\left(\mathbf{C D C l}_{3}, 400 \mathrm{MHz}\right) \delta 0.326(\mathrm{~s}, 6 \mathrm{H}), 2.02-2.09(\mathrm{~m}, 1 \mathrm{H}), 2.22(\mathrm{dd}, J=8.8,15.6 \mathrm{~Hz}, 1 \mathrm{H}), 2.41(\mathrm{dd}, J$ $=5.6,15.6 \mathrm{~Hz}, 1 \mathrm{H}), 2.58-2.70(\mathrm{~m}, 2 \mathrm{H}), 3.20(\mathrm{~s}, 1 \mathrm{H}), 3.57(\mathrm{~s}, 3 \mathrm{H}), 7.35-7.40(\mathrm{~m}, 3 \mathrm{H}), 7.48-7.51(\mathrm{~m}, 2 \mathrm{H})$

${ }^{13} \mathbf{C ~ N M R}\left(\mathbf{C D C l}_{3}, \mathbf{1 0 0} \mathbf{M H z}\right) \delta 186.8,173.5,136.2,133.9,133.9,129.5,128.0,128.0,81.3,78.6,51.6,45.8$, $34.2,17.6,-4.56,-4.61$

HRMS (ESI): $[\mathrm{M}+\mathrm{Na}]^{+}$calcd for $\mathrm{C}_{16} \mathrm{H}_{20} \mathrm{O}_{3} \mathrm{SiNa}: 311.1074$, found: 311.1079

IR(neat) $v 3281,2925,2359,1719,1439,1263,1015,763 \mathrm{~cm}^{-1}$

$[\alpha]_{\mathrm{D}}{ }^{26}-8.80\left(c 0.050, \mathrm{CHCl}_{3}\right)$

$\mathbf{R}_{\mathbf{f}}(n$-Hexane: EtOAc $=2: 1$, color reagent: Hanessian's stain reagent $): 0.60$

\subsection{Reduction \& intramolecular lactonization of compound 16}

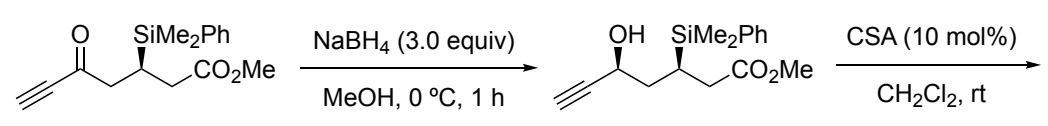

16

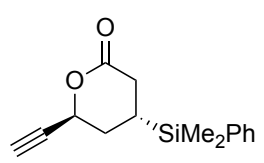

11

To a solution of compound $16(14.4 \mathrm{mg}, 0.050 \mathrm{mmol})$ in $\mathrm{MeOH}(150 \mu \mathrm{L})$, sodium borohydride $(5.68 \mathrm{mg}$, $0.15 \mathrm{mmol}$ ) was added at $0{ }^{\circ} \mathrm{C}$. After stirred at this temperature for 1 hour, the reaction mixture was quenched by sat. aq. ammonium chloride $(0.5 \mathrm{~mL})$. The residue was diluted by EtOAc $(0.5 \mathrm{~mL})$. After separated, the aqueous layer was extracted with EtOAc $(1 \mathrm{~mL})$ for three times. The combined organic layers were washed by brine $(3 \mathrm{~mL})$ and dried over sodium sulfate. The solvent was removed by evaporation under reduced pressure. It was difficult to determine the value of diastereo ratio because the both of peaks were overlap in ${ }^{1} \mathrm{H}-\mathrm{NMR}$ spectrum. To distinguished its peaks, alcohol was converted to lactone. To a solution of the residue in $\mathrm{CH}_{2} \mathrm{Cl}_{2}(0.5 \mathrm{~mL})$, CSA $(2.32 \mathrm{mg}, 0.0020 \mathrm{mmol})$ was added at room temperature. After stirred at this temperature for 1 hour, the residue was directly purified by column chromatography $(n$-Hexane: EtOAc $=5: 1)$ to give diastereomixtures of compound $11(9.8 \mathrm{mg}, 0.038 \mathrm{mmol})$ with $s y n$ : anti $=3: 1$. Syn: anti ratio means that the diastereo ratio of the compound $\mathbf{1 1}$.

\subsection{A side-reaction in asymmetric Michael reaction}




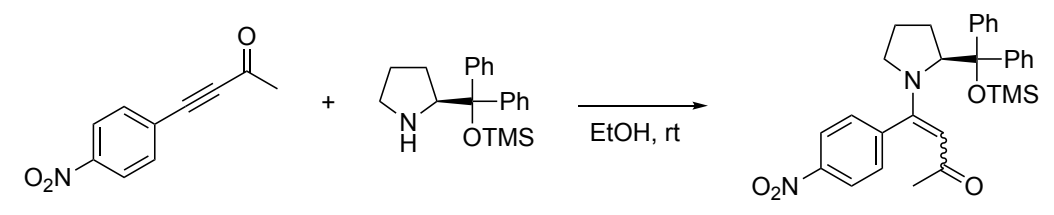

To a solution of 4-(4-nitrophenyl)but-3-yn-2-one $(18.9 \mathrm{mg}, 0.10 \mathrm{mmol})$ in EtOH $(100 \mu \mathrm{L}),(S)-2$ (diphenyl((trimethylsilyl)oxy)methyl)pyrrolidine $(32.5 \mathrm{mg}, 0.10 \mathrm{mmol})$ was added at room temperature. After stirred at this temperature for 1 hour, the reaction mixture was concentrated under reduced pressure. The residue was directly purified by column chromatography on silica gel $\left(n\right.$-Hexane: $\mathrm{EtOAc}=3: 1$ with $\left.1 \% \mathrm{Et}_{3} \mathrm{~N}\right)$ to give adduct $(38.5 \mathrm{mg}, 0.075 \mathrm{mmol})$ in $75 \%$.

(S)-4-(2-(Diphenyl((trimethylsilyl)oxy)methyl)pyrrolidin-1-yl)-4-(4-nitrophenyl)but-3-en-2-one<smiles>CC(=O)C=C(c1ccc([N+](=O)[O-])cc1)N1CCC[C@H]1C(O[Na])(c1ccccc1)c1ccccc1</smiles>

E/Z mixtures

Yield: $75 \%(38.5 \mathrm{mg})$

Physical State: Yellow oil

${ }^{1}$ H NMR $\left(\mathbf{C D C l}_{3}, 400\right.$ MHz) $\delta$-0.15 (s, 9H), 0.83-0.93 (m, 1H), 1.21-1.28 (m, 1H), 1.85 (s, 3H), 1.93-2.04 (m, 2H) 2.15-2.22 (m, 1H), 3.47 (ddd, $J=5.6,9.6,11.2 \mathrm{~Hz}, 1 \mathrm{H}), 4.80$ (dd, $J=7.2,9.2 \mathrm{~Hz}, 1 \mathrm{H}), 5.40$ (brs, $1 \mathrm{H}), 7.29-7.43(\mathrm{~m}, 10 \mathrm{H}), 8.00-8.19(\mathrm{~m}, 4 \mathrm{H})$

${ }^{13} \mathrm{C}$ NMR $\left(\mathbf{C D C l}_{3}, \mathbf{1 0 0} \mathrm{MHz}\right) \delta 193.9,163.0,158.8,158.8,147.3,147.3,142.3,142.1,141.4,129.3,129.3$, 129.3 , 129.3, 128.1, 128.1, 127.8, 127.8, 127.7, 127.7, 127.5, 127.5, 83.2, 66.1 44.0, 30.8, 27.3, 22.1, 2.09, $2.09,2.09$

HRMS (ESI): [M+Na] $]^{+}$calcd for $\mathrm{C}_{30} \mathrm{H}_{34} \mathrm{~N}_{2} \mathrm{O}_{4} \mathrm{SiNa}$ : 537.2180, found: 537.2180

$\operatorname{IR}\left(\right.$ neat) $v 2956,1661,1601,1523,1493,1345,1252,1182,1069,969,840,755,704 \mathrm{~cm}^{-1}$

$\mathbf{R}_{\mathbf{f}}(n$-Hexane: EtOAc $=1: 2$, color reagent: Hanessian's stain reagent $): 0.10$

\subsection{Compounds information about side-product}

(4E,6Z)-7-Phenyl-1-(triisopropylsilyl)hepta-4,6-dien-1-yn-3-one (4)<smiles>O=C(C#CSP)C=CC=Cc1ccccc1</smiles>

$\mathrm{E} / \mathrm{Z}$ mixtures. (The presented structure is major isomer determined by coupling constant.)

Physical State: Brawn oil

${ }^{1}$ H NMR $\left(\mathbf{C D C l}_{3}, \mathbf{4 0 0}\right.$ MHz) $\delta$ 0.96-1.04 (m, 21H), 6.35 (d, $\left.J=15.2 \mathrm{~Hz}, 1 \mathrm{H}\right), 6.96-6.97$ (m, 2H) 7.32-7.44 
(m, 3H), 7.49-7.52 (m, 2H), $7.67(\mathrm{ddd}, J=3.6,6.8,15.6 \mathrm{~Hz}, 1 \mathrm{H})$

${ }^{13} \mathrm{C}$ NMR $\left(\mathbf{C D C l}_{3}, \mathbf{1 0 0} \mathrm{MHz}\right) \delta 177.8,148.4,142.7,135.7,131.9,129.6,128.9,128.9,127.5,127.5,126.4$, $102.9,95.7,18.6,18.6,18.6,18.6,18.6,18.6,11.1,11.1,11.1$

HRMS (ESI): [M+Na] ${ }^{+}$calcd for $\mathrm{C}_{22} \mathrm{H}_{30} \mathrm{OSiNa}$ : 361.1958, found: 361.1959

IR(neat) $v 2946,2866,2151,1615,1462,1231,1105,997,881,754,679 \mathrm{~cm}^{-1}$

$\mathbf{R}_{\mathbf{f}}(n$-Hexane: EtOAc $=3: 1$, color reagent: Hanessian's stain reagent $): 0.85$

(1'R,2' $\left.S, 3^{\prime} R\right)$-2' -(3-(Triisopropylsilyl)propioloyl)-1',2',3',6'-tetrahydro-[1,1':3',1' '-terphenyl]-4'carbaldehyde (5)<smiles>O=CC1=CC[C@@H](c2ccccc2)[C@H](C(=O)C#CS[In])[C@H]1c1ccccc1</smiles>

Diastereomeric mixtures. (The presented structure is major isomer determined by coupling constant.)

Physical State: Brawn oil

${ }^{1} \mathrm{H}$ NMR $\left(\mathbf{C D C l}_{3}, \mathbf{4 0 0} \mathrm{MHz}\right) \delta$ 0.96-1.04 (m, 21H), $2.46(\mathrm{tdd}, J=2.4,10.4,21.2 \mathrm{~Hz}, 1 \mathrm{H}), 3.01(\mathrm{td}, J=5.6$, $21.2 \mathrm{~Hz}, 1 \mathrm{H}), 3.46(\mathrm{dd}, J=4.8,12.4 \mathrm{~Hz}, 1 \mathrm{H}), 3.52(\mathrm{ddd}, J=5.4,10.8,12.4 \mathrm{~Hz}, 1 \mathrm{H}), 4.58(\mathrm{~d}, J=4.8 \mathrm{~Hz}, 1 \mathrm{H})$, $7.08(\mathrm{t}, J=1.2 \mathrm{~Hz}, 1 \mathrm{H}), 7.14-7.33(\mathrm{~m}, 10 \mathrm{H}), 9.47(\mathrm{~s}, 1 \mathrm{H})$

${ }^{13} \mathrm{C}$ NMR $\left(\mathrm{CDCl}_{3}, \mathbf{1 0 0} \mathrm{MHz}\right) \delta$ 204.8, 191.6, 149.2, 143.4, 141.8, 137.4, 129.3, 129.3, 128.7, 128.7 128.4, 128.4 , 127.3 , 127.3 , 126.7, 126.7, 104.2, 97.5, 56.8, 41.3, 36.7, 36.0, 18.5, 18.5, 18.5, 18.4 , 18.4, 18.4, 10.9, $10.9,10.9$

HRMS (ESI): [M+Na] $]^{+}$calcd for $\mathrm{C}_{31} \mathrm{H}_{38} \mathrm{O}_{2} \mathrm{SiNa}$ : 493.2533, found: 493.2538

IR(neat) $v$ 3428, 3030, 2945, 2866, 2146, 1726, 1685, 1494, 1460, 1374, 1204, 1148, 1074, 997, 957, 882, $758,701,583 \mathrm{~cm}^{-1}$

$\mathbf{R}_{\mathbf{f}}(n$-Hexane: EtOAc $=3: 1$, color reagent: Hanessian's stain reagent $): 0.20$

\section{References}

[1] Y. Gu, B. B. Snider, Org. Lett. 2003, 5, 4385. 


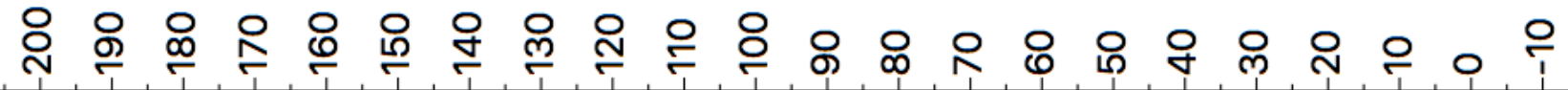

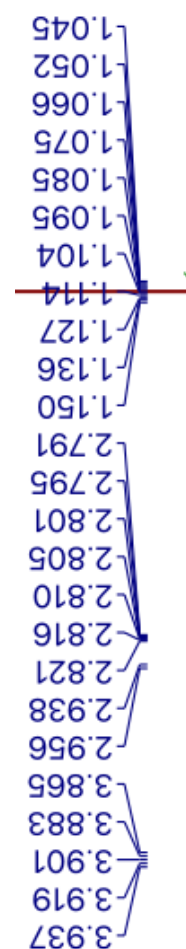

\section{6l' $L$}

$\left.\angle L Z^{\circ} \angle\right]$

$092 \angle$

$98 Z \angle \mathcal{C}$

$\varepsilon 6 Z^{\circ} \angle$

$\varepsilon 0 \varepsilon^{\circ} \angle$

$\varepsilon 乙 \varepsilon^{*}\llcorner$

$\angle 99^{\circ} 6$

$299^{\circ} 6>$

$999^{\circ}$
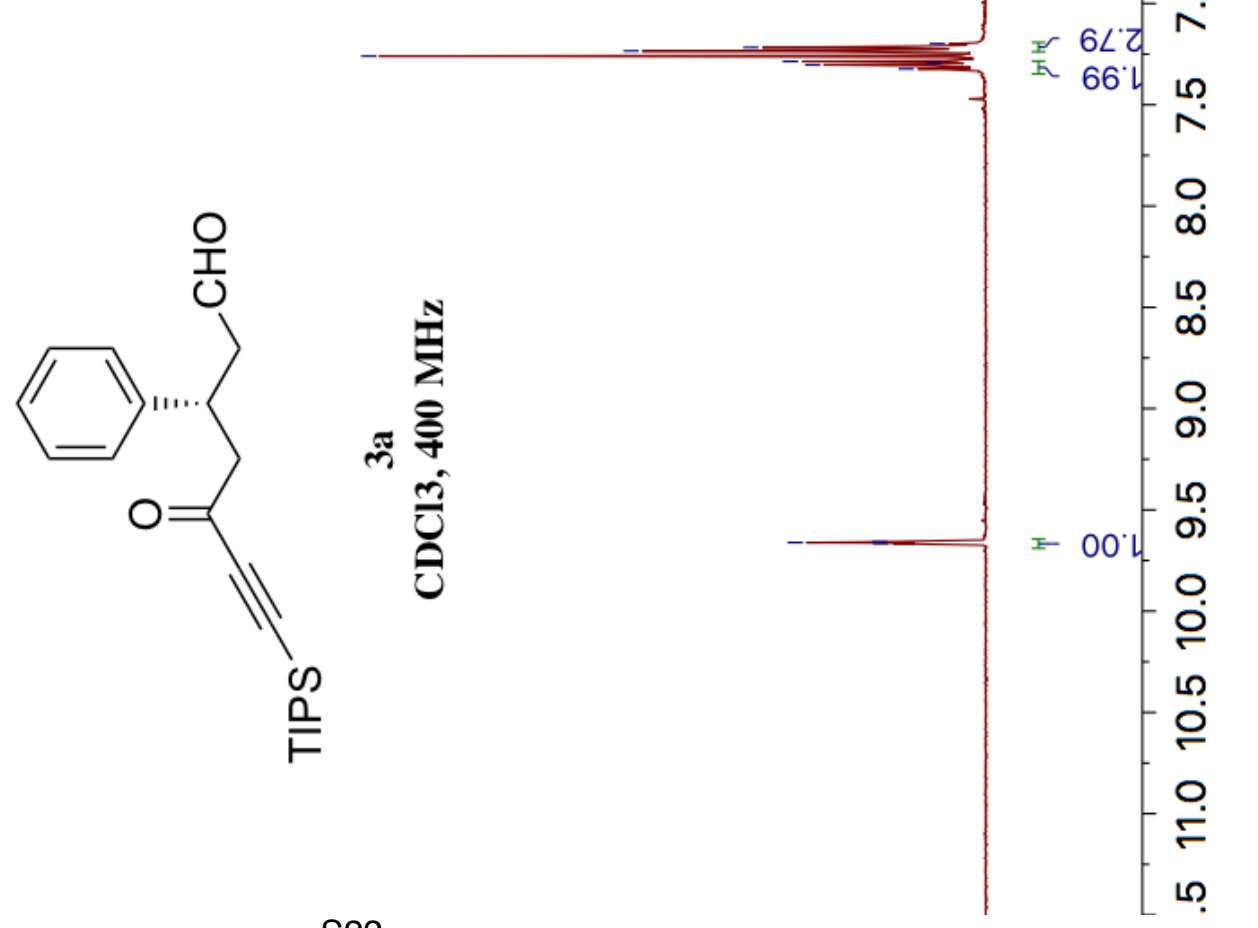


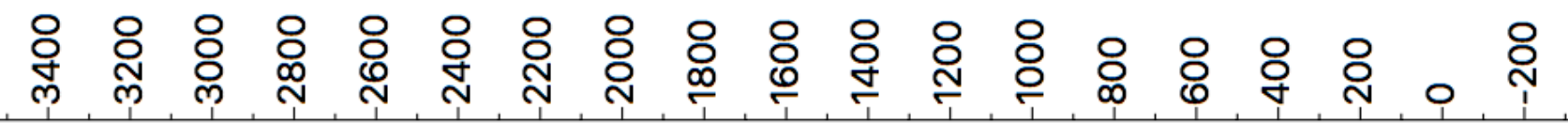

8†6.0L-

,

$69 t^{\circ} 8 \mathrm{~L}$

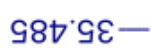

$\angle 8 Z^{\circ} 67-$

009 'L -

189.94

$000 \angle L J$

$8 \mathrm{~L} \varepsilon^{\circ} L L^{\prime}$

$\varepsilon 89^{\circ} 96-$

$\angle L O \circ O L-$

$6 Z L: \angle Z L]$

$9 \succ \varepsilon \cdot \angle Z L$

$\rightarrow 98.82 \mathrm{~L}$

งEl`てヤ -

ยZІ`ง8เ-

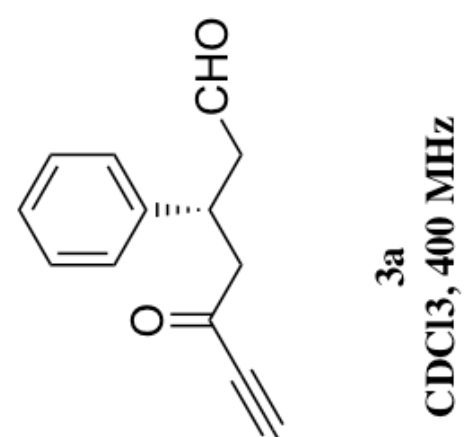

$87 t 00 z-$

$\omega$

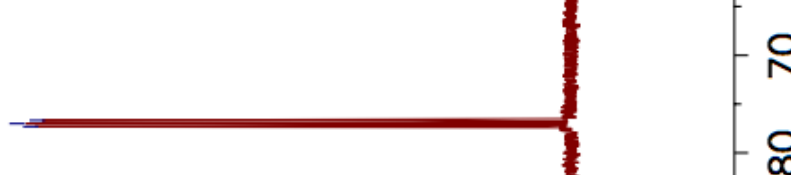

요

오

- 응

을 흥

윤

으

원

은

음

을

음

음

으

읃 


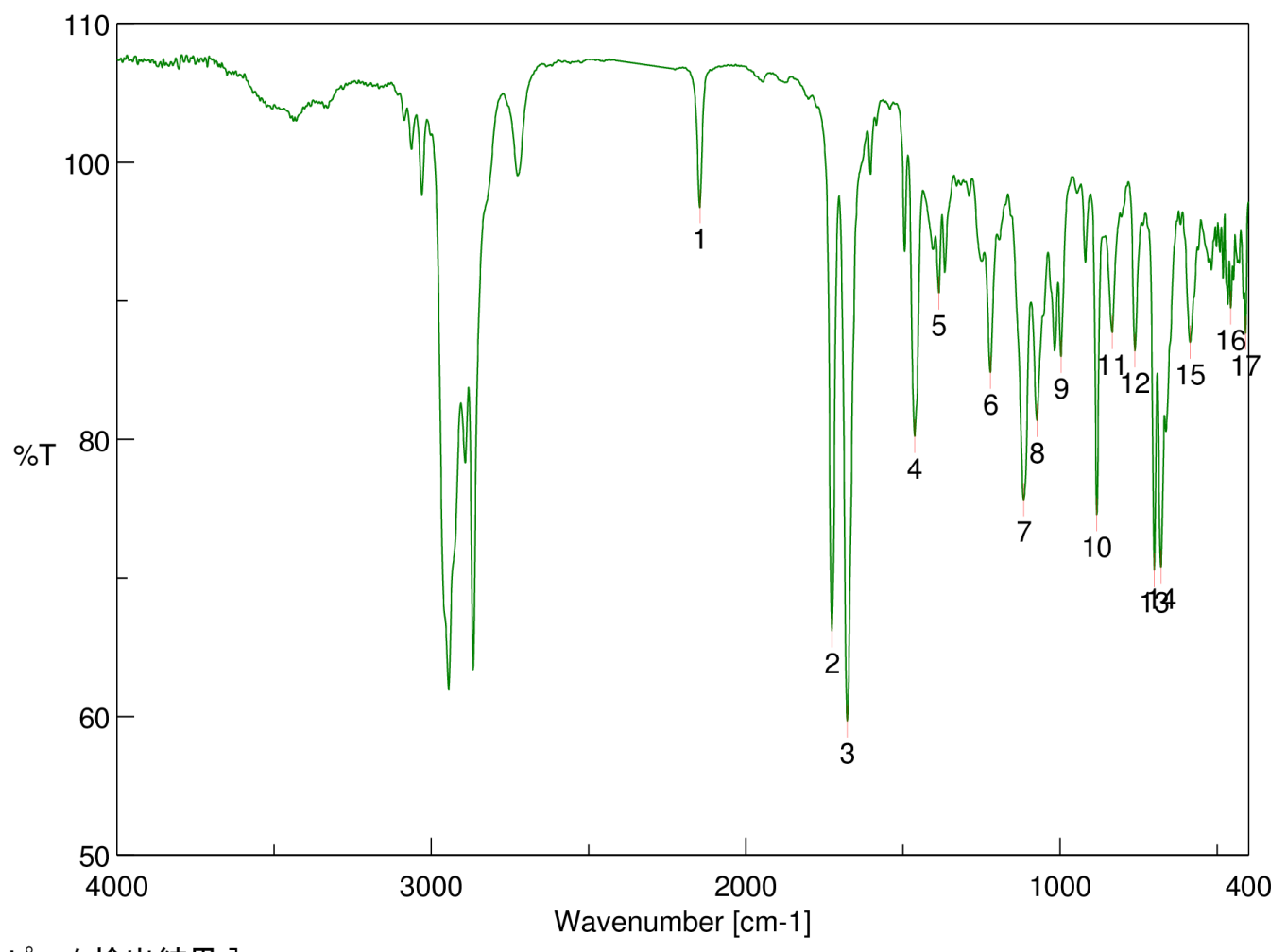

[ピーク検出結果 ]

$\begin{array}{llllll}\text { No. } & \text { 位置 } & \text { 強度 } & \text { No. } & \text { 位置 } & \text { 強度 } \\ 1 & 2145.42 & 96.7798 & 2 & 1725.98 & 66.1811 \\ 3 & 1676.8 & 59.6778 & 4 & 1462.74 & 80.2008 \\ 5 & 1385.6 & 90.5405 & 6 & 1221.68 & 84.8352 \\ 7 & 1115.62 & 75.6454 & 8 & 1073.19 & 81.371 \\ 9 & 997.017 & 85.9852 & 10 & 883.238 & 74.5744 \\ 11 & 834.062 & 87.7047 & 12 & 761.744 & 86.3906 \\ 13 & 700.034 & 70.576 & 14 & 678.82 & 70.8001 \\ 15 & 586.254 & 87.0057 & 16 & 457.047 & 89.4714 \\ 17 & 409.799 & 87.6601 & & & \end{array}$<smiles>O=CC[C@H](CC(=O)C#C[InH])c1ccccc1</smiles> 


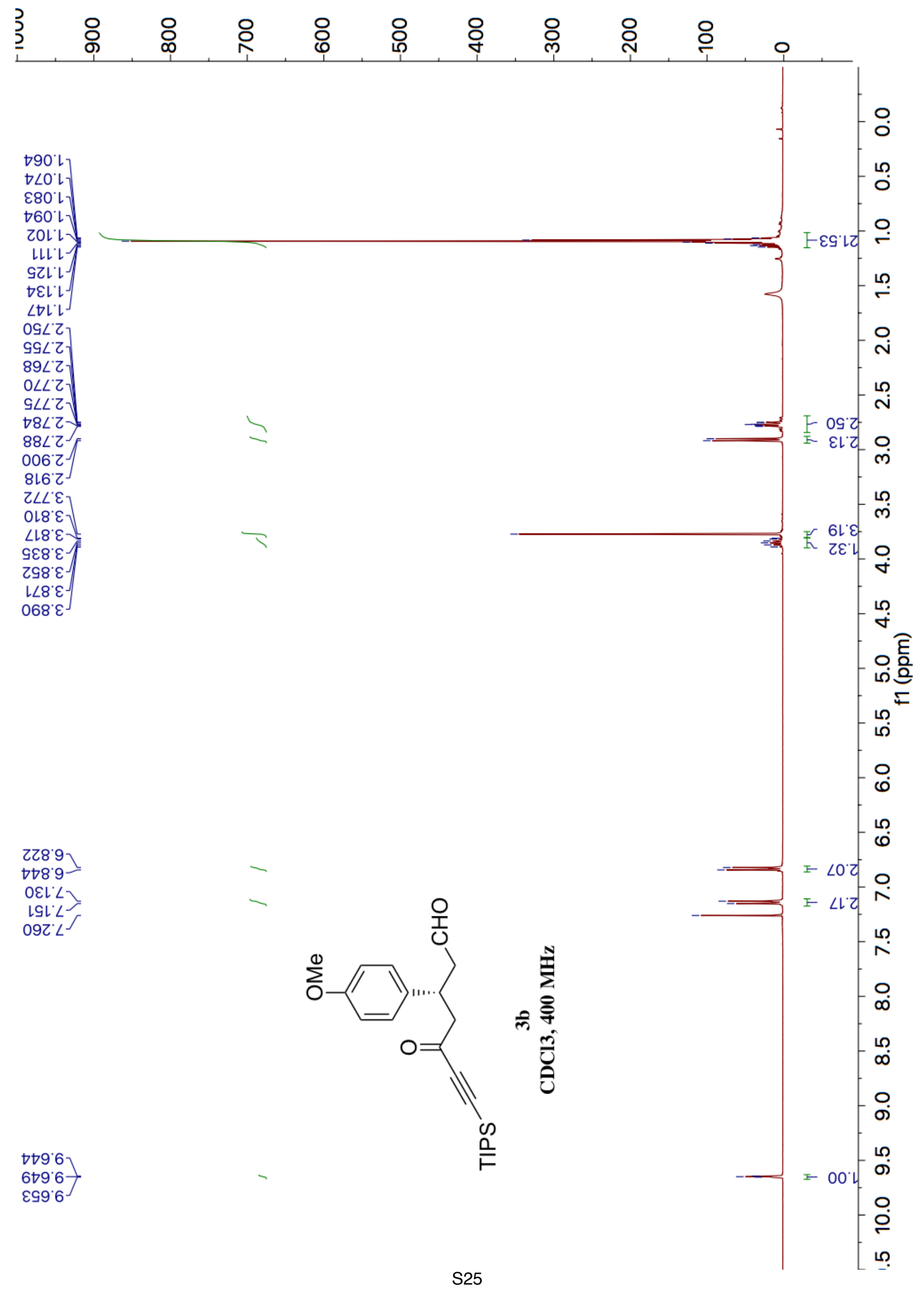




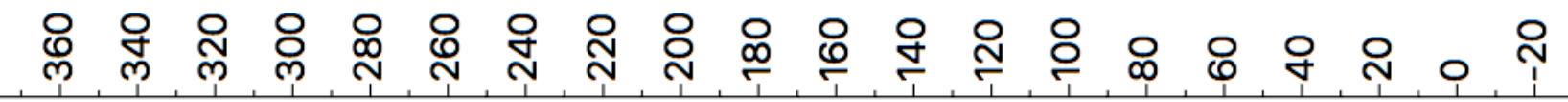

196.0L-

$997^{\circ} 81$

$\llcorner\angle 8 \vee \downarrow \varepsilon-$

$0 \angle D^{\circ} 6 \mathrm{~T} \longrightarrow$

$\angle 98^{\circ}$ LS -

LIZGG

$189 \cdot 94$

$000 \angle L$

$8 \mathrm{~L} \varepsilon^{\prime} L L^{\prime}$

tl9.96-

$280 \div 0\llcorner-$

6てでャルー

$\angle ৮ \varepsilon^{\circ} 8 Z \downarrow-$

$\mathrm{S} \angle 0 \circ \varepsilon L-$

t99.89L-

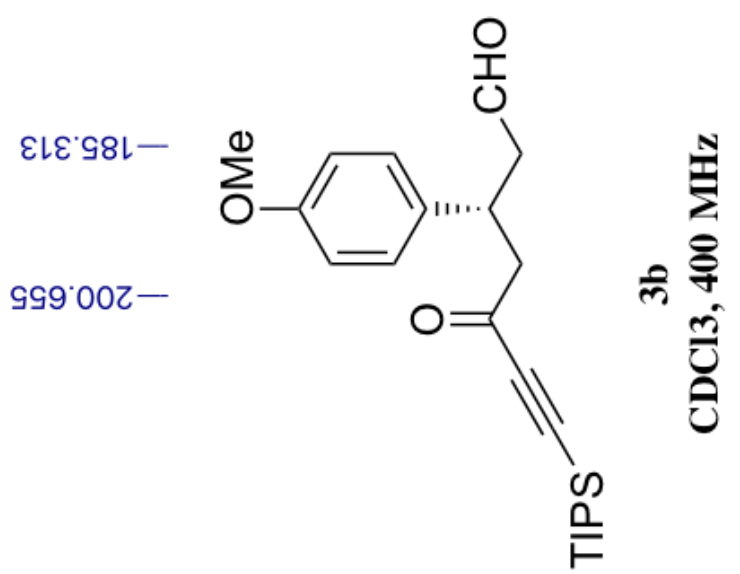




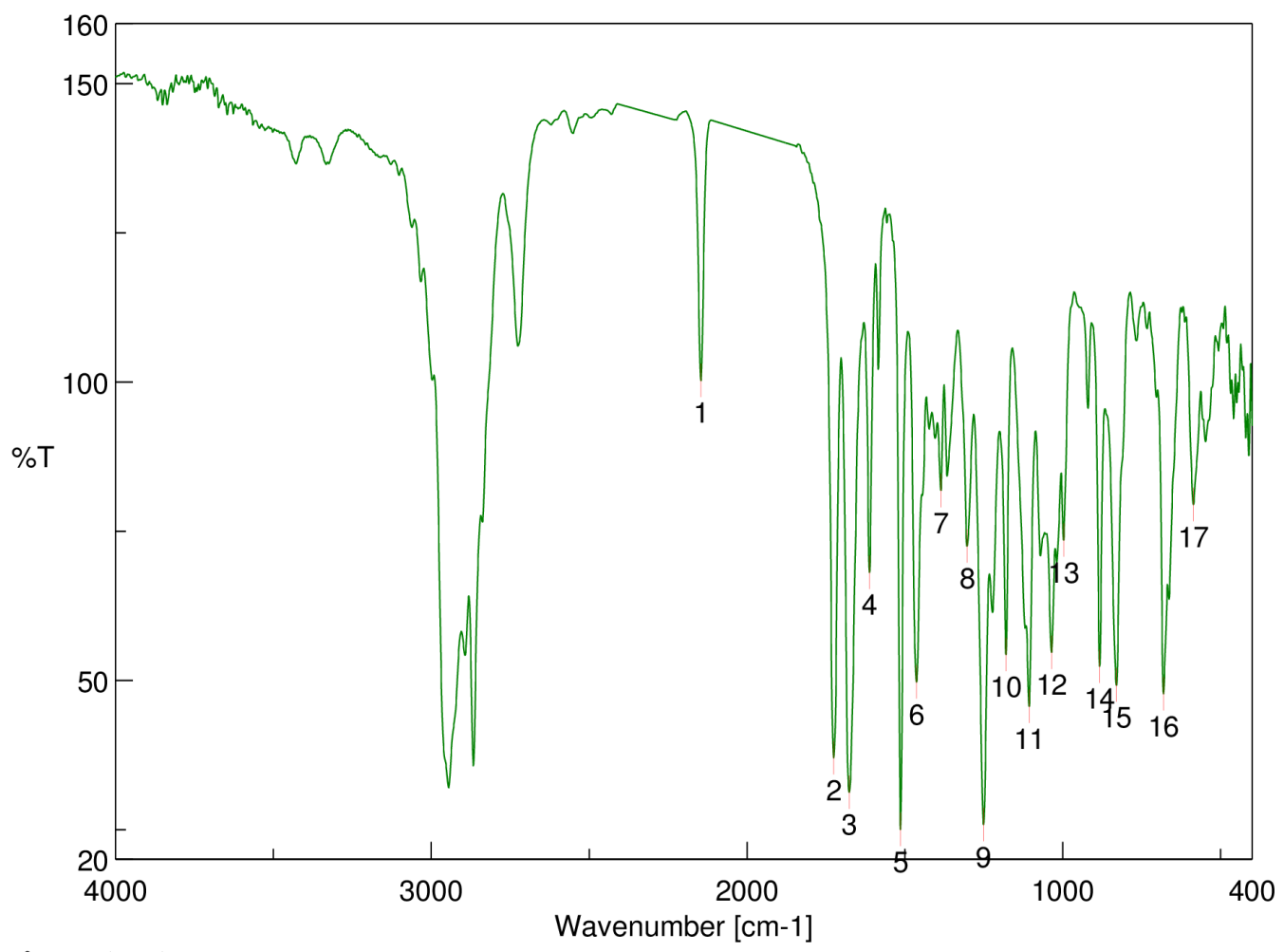

\section{[ピーク検出結果 ]}

$\begin{array}{llllll}\text { No. } & \text { 位置 } & \text { 強度 } & \text { No. } & \text { 位置 } & \text { 強度 } \\ 1 & 2146.38 & 100.199 & 2 & 1725.01 & 36.9878 \\ 3 & 1675.84 & 31.2231 & 4 & 1612.2 & 68.0906 \\ 5 & 1513.85 & 25.0181 & 6 & 1463.71 & 49.7064 \\ 7 & 1385.6 & 81.7601 & 8 & 1302.68 & 72.4826 \\ 9 & 1250.61 & 25.8514 & 10 & 1179.26 & 54.3048 \\ 11 & 1105.98 & 45.6517 & 12 & 1035.59 & 54.6377 \\ 13 & 997.017 & 73.504 & 14 & 883.238 & 52.306 \\ 15 & 830.205 & 49.1953 & 16 & 680.749 & 47.7322 \\ 17 & 586.254 & 79.4357 & & & \end{array}$<smiles>COc1ccc([C@H](CC=O)CC(=O)C#CC[AsH2+]C(C)C)cc1</smiles> 
孚 운

$190^{\circ} \mathrm{L}$

$0 \angle 0^{\circ} \mathrm{L}$

$6 \angle 0^{\circ} \mathrm{L}$

$060^{\circ} \mathrm{L}$

$660^{\circ} \mathrm{L}$

$801 \%$

$8 \angle L ' Z$

$\mathrm{Z} 8 L^{\circ} \mathrm{Z}$

$\mathrm{Z08} \mathrm{Z}$

918'

$618^{\circ} \mathrm{Z}$

706 '

0เ6'

\&Z6 乙

8Z6

0Z8 ${ }^{\prime} \varepsilon$

$8 \varepsilon 8^{\prime} \varepsilon$

998' $\varepsilon-$

$\nabla \angle 8^{\circ} \varepsilon$

乙68 ${ }^{\circ}$

OOL'L

GOL: $\angle$

$\angle L L \angle \sqrt{2}$

09Z: $\angle$

tレเ $\angle J$

6LV $L$

OEt $L$

set $L$

999.6

$899^{\circ} 6$

$299^{\circ}$

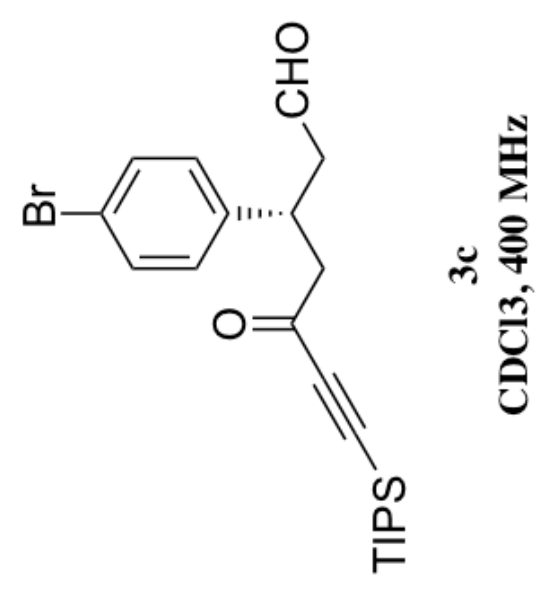

S28
26.

ले

ח

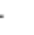

+

ช

응

F

เก

$\circ$

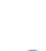

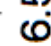

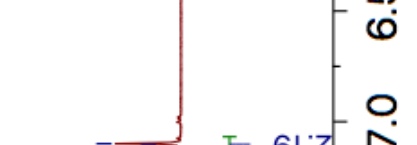

6120

F $\varepsilon 0^{\circ} z$.

$\stackrel{2}{\wedge}$

$\stackrel{\circ}{\infty}$

$\stackrel{\infty}{\infty}$

우

ก

F 00.

웅

눙 
品 운

S96:0L-

แLG'8L-

$006{ }^{\circ} \nabla \varepsilon-$

$69 l^{\circ} 6 \mathrm{~b}-$

89Z'LG-

$289.9 L$

$000 \angle L T$

$8 \mathrm{~L} \varepsilon^{\prime} L L^{\prime}$

E89.'6-

Z96' $\mathrm{EOL}-$

896.0Zl-

ยgl'6Zl -

IS6'LEL-

60Z゙เレーー

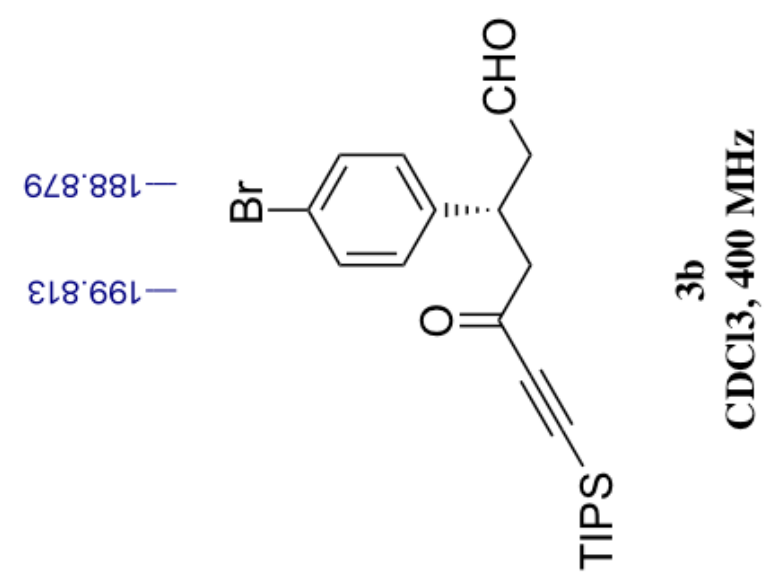




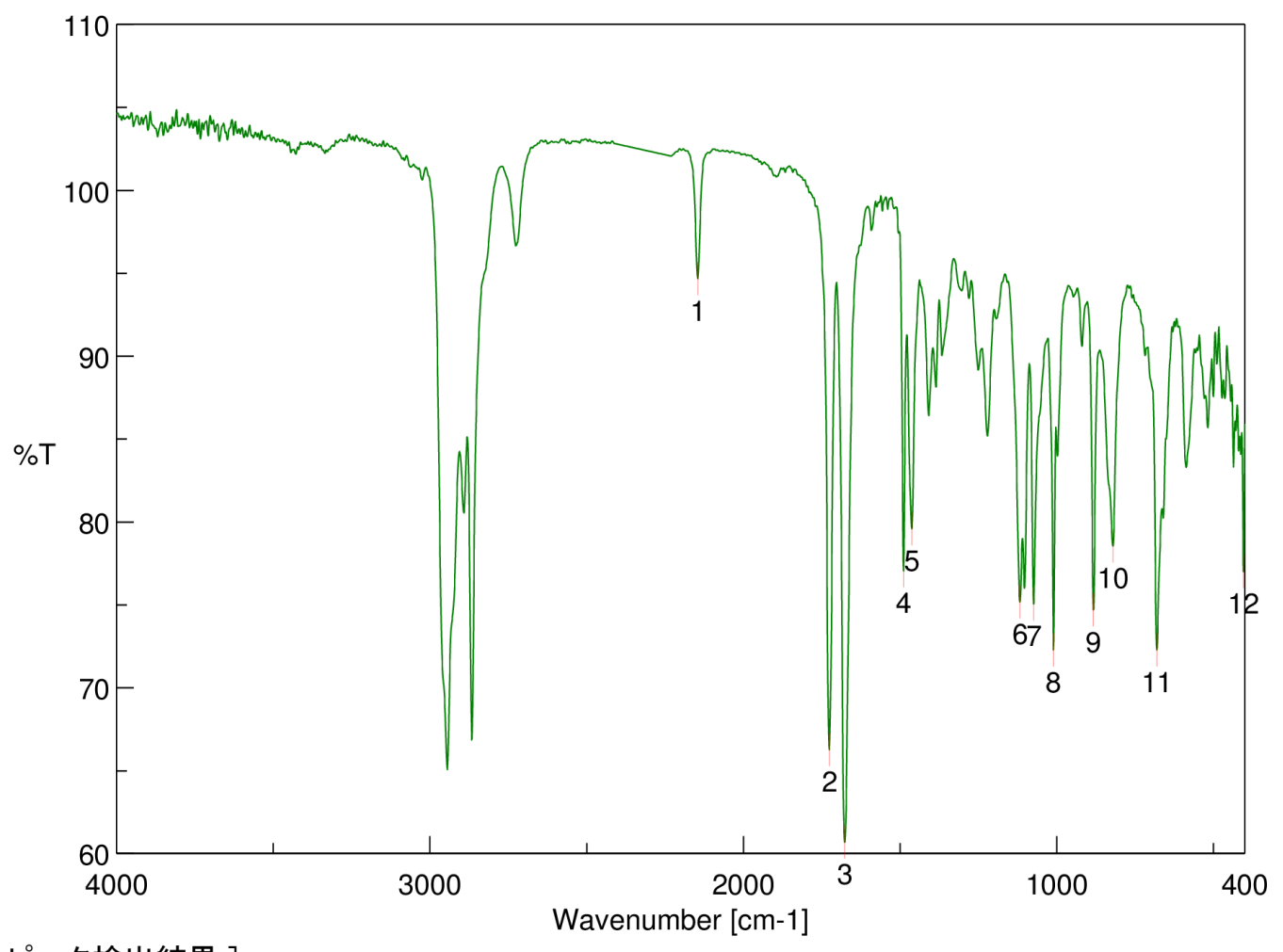

[ピーク検出結果 ]

$\begin{array}{llllll}\text { No. } & \text { 位置 } & \text { 強度 } & \text { No. } & \text { 位置 } & \text { 強度 } \\ 1 & 2145.42 & 94.6656 & 2 & 1725.98 & 66.2595 \\ 3 & 1676.8 & 60.68 & 4 & 1488.78 & 77.0398 \\ 5 & 1462.74 & 79.5739 & 6 & 1117.55 & 75.1742 \\ 7 & 1074.16 & 75.0461 & 8 & 1010.52 & 72.2698 \\ 9 & 883.238 & 74.6965 & 10 & 820.563 & 78.5467 \\ 11 & 680.749 & 72.2851 & 12 & 404.014 & 77.0076\end{array}$<smiles>O=CC[C@H](CC(=O)C#CS[In])c1ccc(Br)cc1</smiles> 


\section{当道}

$990^{\circ} \mathrm{L}$

$690^{\circ} \mathrm{L}$

$8 \angle 0^{\circ} \mathrm{L}$

$\angle 80^{\circ} \mathrm{L}$

$860^{\circ} \mathrm{L}$

$\left.80 L^{\circ} \cdot\right]$

ZEL

Otl.L

$\nabla G l \cdot L$

S9l.

$\forall \angle L \cdot L$

$16 \angle ' Z$

$\mathrm{s} 6 \angle 2 \mathrm{Z}$

908'Z

608 ' 2

$\operatorname{ti8} Z$

$Z Z 8^{\circ} 乙$

ตZ8'

$916 \cdot 2$

$6 เ 6 \cdot 2$

$\nabla \varepsilon 6^{\circ} Z$

$9 \varepsilon 6{ }^{\prime} \tau^{-}$

$8 Z 8^{\circ} \varepsilon$

978 '

$\supset 98^{\circ} \varepsilon$

乙88' $\varepsilon$

$006^{\prime} \varepsilon$

$\mathrm{Z9l}: \mathrm{L}$

S9l' $\angle$

$0 \angle l^{\circ} \angle$

$\nabla \angle L \angle$

$8 \angle l^{\circ} \angle \sqrt{ }$

$0 \succ \varepsilon\llcorner$

St $\varepsilon \angle$

$8 \nabla \varepsilon<\mathcal{C}$

SSE $L$

$\angle G E^{\circ} \angle$

$\varepsilon 9 \varepsilon^{\circ} L$

$89 \varepsilon^{\circ} \angle-$

$G \angle \varepsilon^{\circ} \angle$

$999^{\circ} 6$

$6996-$

Z $\angle 96^{\circ}$
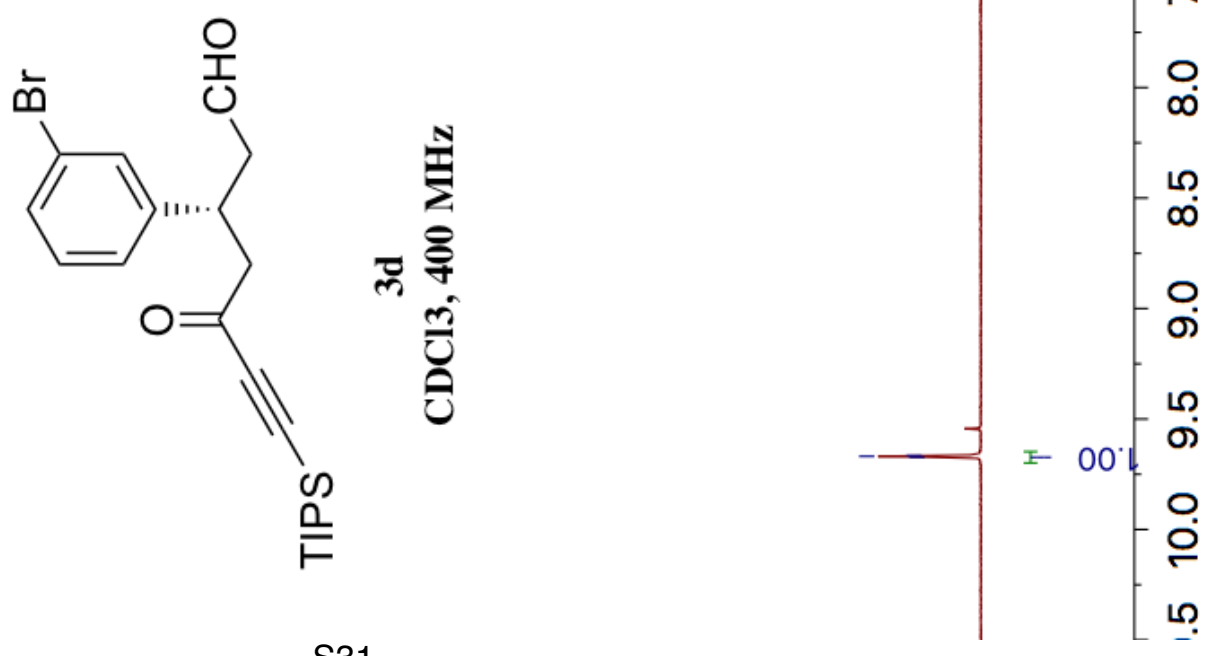


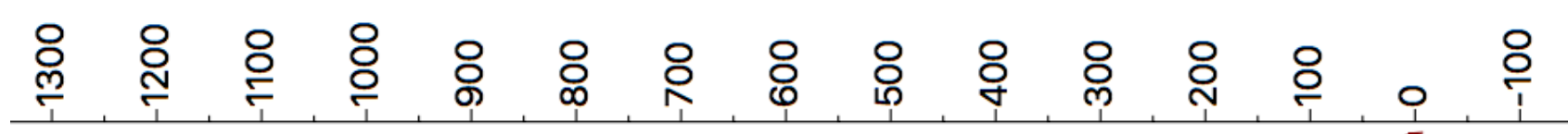

$896.01-$

$\mathrm{s} \angle t \cdot 8 \mathrm{~L}-$

ง00`ง -

Etเ'6t

6LI'LS

$189^{\circ} 9 L$

$000 \angle L-$

$8 \mathrm{~L} \varepsilon^{\prime} L L$

$\angle 60 \angle 6-$

${ }^{\circ} 06^{\circ} \mathrm{EOL}-$

068'ZZL

G8l'9Zl

OLE'0हL

†Ot'0हL-

89t0 $0 \varepsilon$.

ยเ9๋カtー

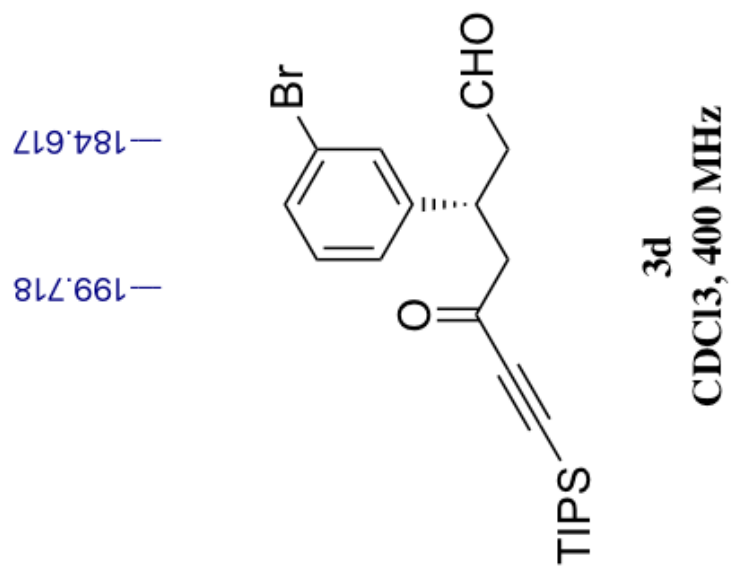

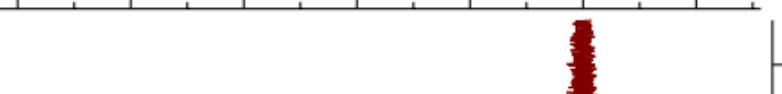

+ 운

두

o

으

요

유

우

웅

8

인

$\infty$

8

-

을 응

$\stackrel{\mathrm{i}}{\mathbb{E}}^{\mathrm{t}}$

음

옹

움

$\stackrel{8}{\circ}$

옹

$\stackrel{\circ}{\circ}$

음

으

은

ิำ

్ㅠ 


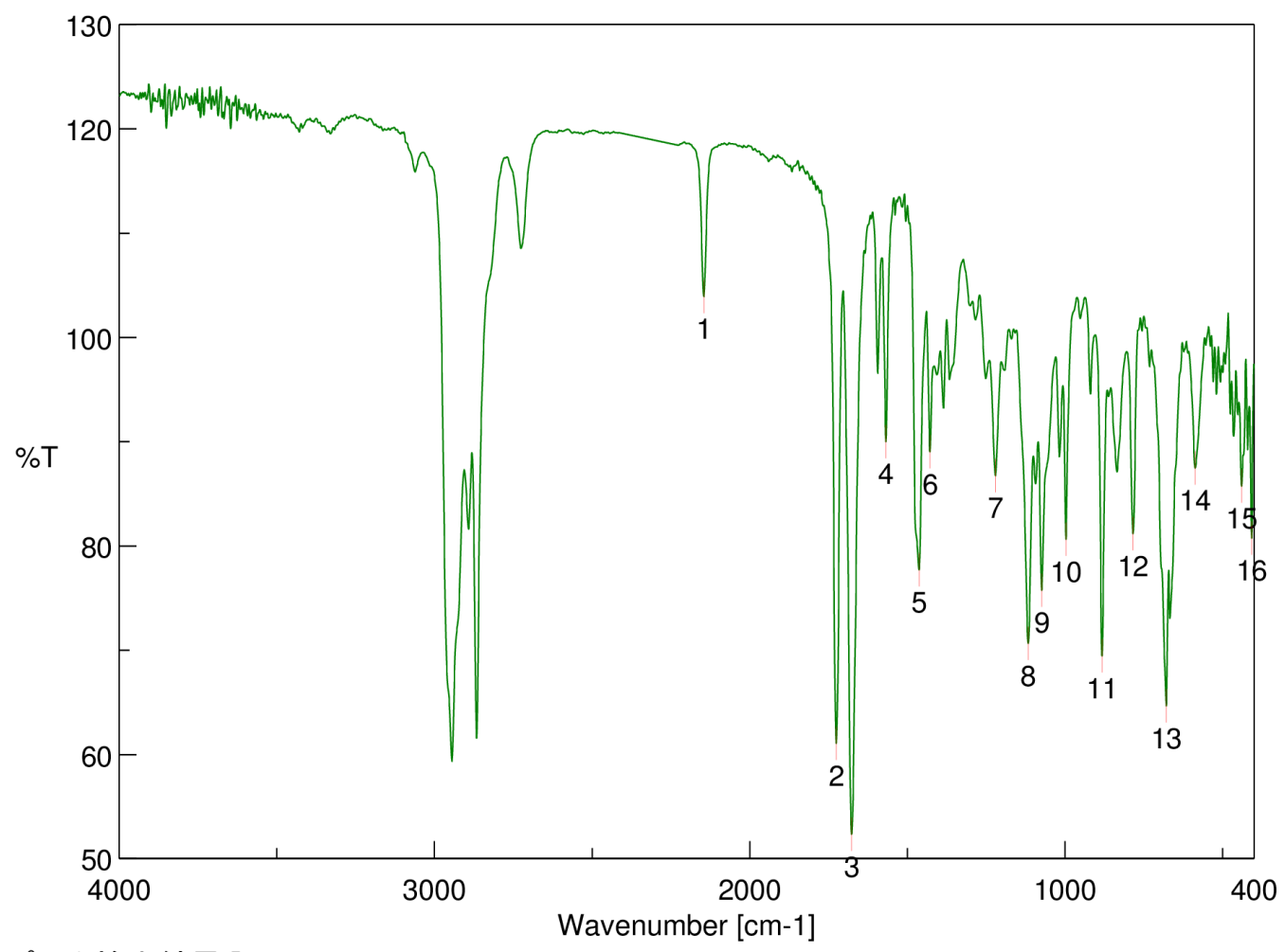

[ピーク検出結果 ]

$\begin{array}{llllll}\text { No. } & \text { 位置 } & \text { 強度 } & \text { No. } & \text { 位置 } & \text { 強度 } \\ 1 & 2145.42 & 103.905 & 2 & 1725.98 & 61.0608 \\ 3 & 1676.8 & 52.3179 & 4 & 1567.84 & 89.9908 \\ 5 & 1462.74 & 77.6846 & 6 & 1428.03 & 89.0289 \\ 7 & 1220.72 & 86.7059 & 8 & 1116.58 & 70.6369 \\ 9 & 1074.16 & 75.7458 & 10 & 997.017 & 80.6148 \\ 11 & 882.274 & 69.4399 & 12 & 783.922 & 81.1542 \\ 13 & 678.82 & 64.6436 & 14 & 587.218 & 87.4619 \\ 15 & 439.69 & 85.7071 & 16 & 407.871 & 80.7341\end{array}$<smiles>O=CCC(CC(=O)C#CC[In])c1cccc(Br)c1</smiles> 


\section{惫总员}

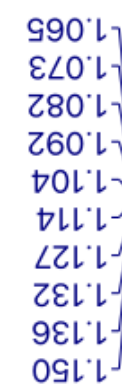

$8 \varepsilon 8 \cdot 7$

Z†8'

५९८'

0982

แL6' 2

เع6' 2

$\varepsilon 96{ }^{\prime} Z$

$\varepsilon \angle 6 \sigma^{\circ} \tau^{-}$

$000^{\circ} \varepsilon$

$\mathrm{SlO} \varepsilon$

乙†० $\varepsilon$

$\angle G 0^{\circ} \varepsilon$

$8 \varepsilon \varepsilon^{\prime} t$

ง

$\varepsilon \angle \varepsilon^{\prime} \circ$

เ6ع' $\nabla$

$60 t^{\circ} t$

$0 \angle 0^{\circ} \angle$

$9 \angle 0 \angle$

$060^{\circ} \angle$

$\varepsilon 60^{\circ} \mathrm{L}$

$80 L^{\prime} L$

Ell: $\angle$

$\angle 6 L: \angle]$

$10 Z \angle]$

$9 l Z \angle$

IZZ: $\angle$

$\angle G Z \angle$

$09 Z \angle$

$9 \angle Z^{\circ} \angle$

$6 \angle Z \angle$

$962 \angle$

$86 Z^{\prime} L$

999' $L$

699' $\angle-$

$9 \angle S^{\circ} L$

$6 \angle S^{\circ} \angle$

$\nabla \angle 96$

$8 \angle 96-$

ع89.6

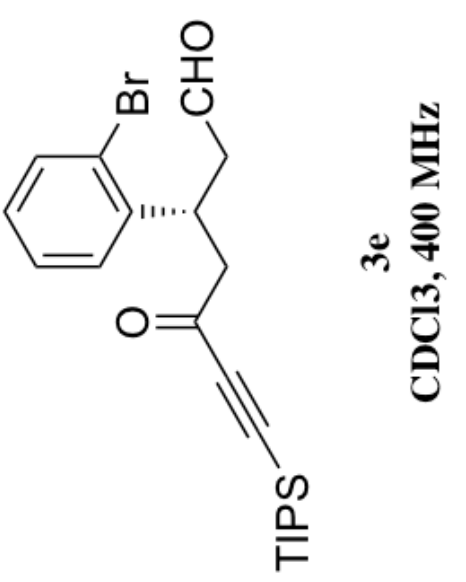

웅 


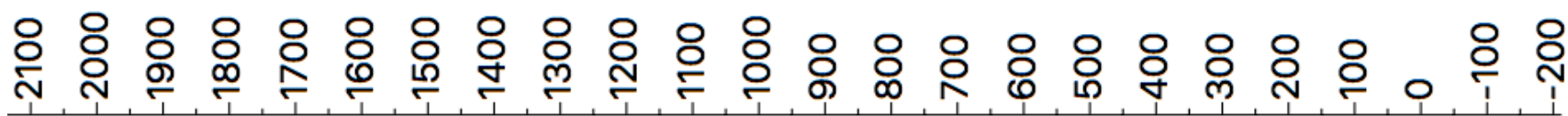

†96.OL-

t

$\angle L \nabla \nabla \varepsilon-$

$926 \angle b>$

$66 L^{\prime} 6 \nabla-$

$189^{\circ} 9 L$

$000 \angle L T$

$8 L \varepsilon^{\prime} L L$

968'96-

$9 \varepsilon 8^{\circ} \varepsilon 0 \mathrm{-}$

$\varepsilon \varepsilon \varepsilon^{\circ} \triangleright Z \downarrow ح$

LZ6 $\angle Z L>$

990 8ZL

I99'8ZL-

เOG'EहL -

86L'0tレ

$08\llcorner\circ \nabla 81-$

แL'00Z-

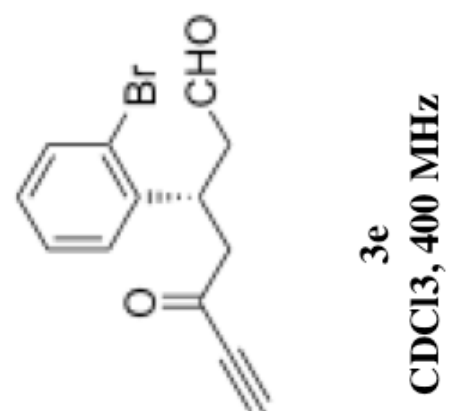

党

$\stackrel{\infty}{\unrhd}$

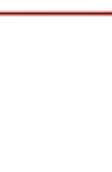

- ㄱ

-

우

웅

8

웅

$\infty$

৪

8 


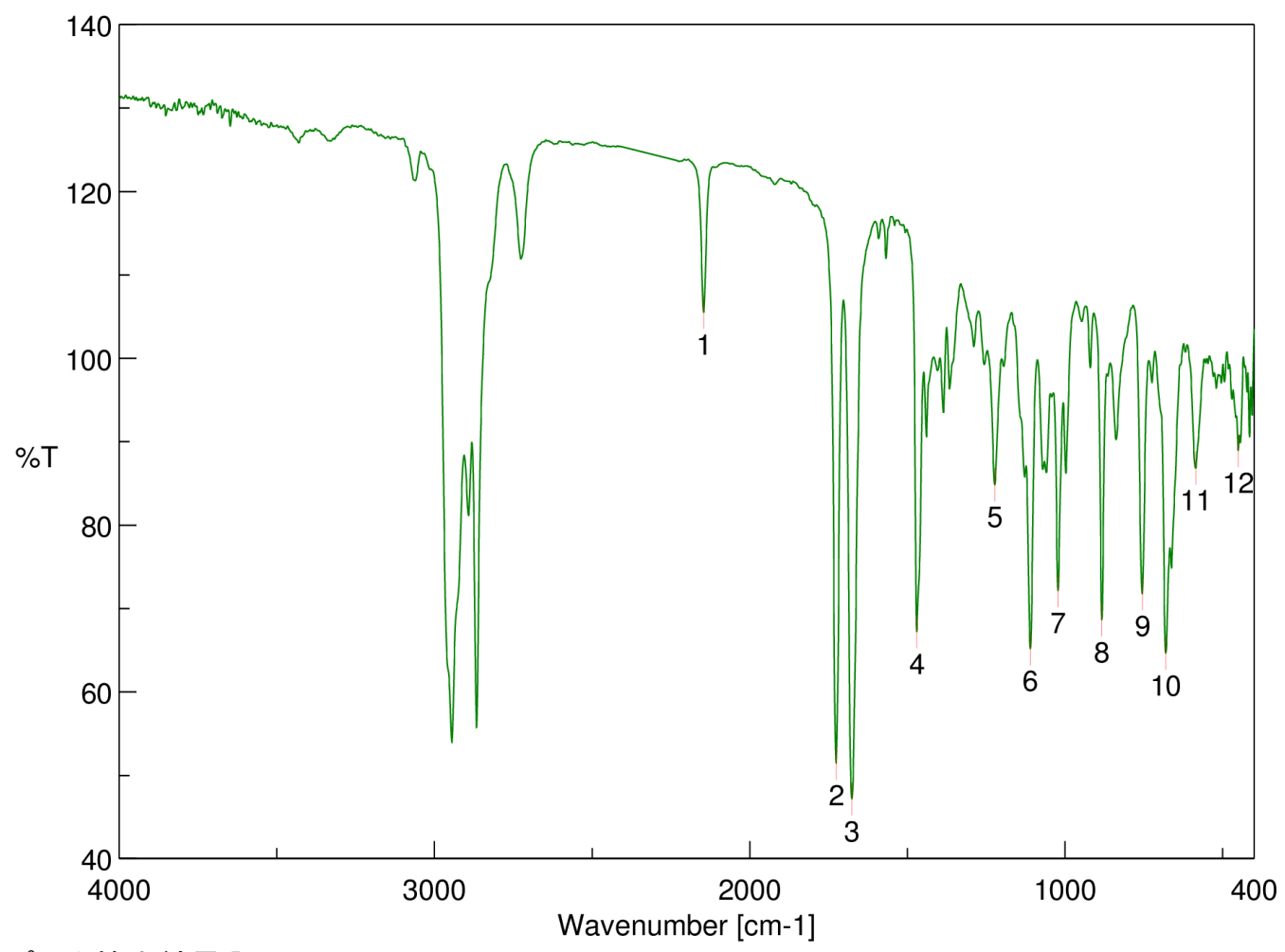

[ピーク検出結果 ]

$\begin{array}{llllll}\text { No. } & \text { 位置 } & \text { 強度 } & \text { No. } & \text { 位置 } & \text { 強度 } \\ 1 & 2146.38 & 105.491 & 2 & 1725.98 & 51.4454 \\ 3 & 1675.84 & 47.1698 & 4 & 1470.46 & 67.212 \\ 5 & 1222.65 & 84.8335 & 6 & 1109.83 & 65.1583 \\ 7 & 1022.09 & 72.1303 & 8 & 883.238 & 68.6319 \\ 9 & 754.995 & 71.7331 & 10 & 680.749 & 64.6486 \\ 11 & 585.29 & 86.8128 & 12 & 450.297 & 88.9388\end{array}$<smiles>O=CC[C@H](CC(=O)C#CS[In])c1ccccc1Br</smiles> 


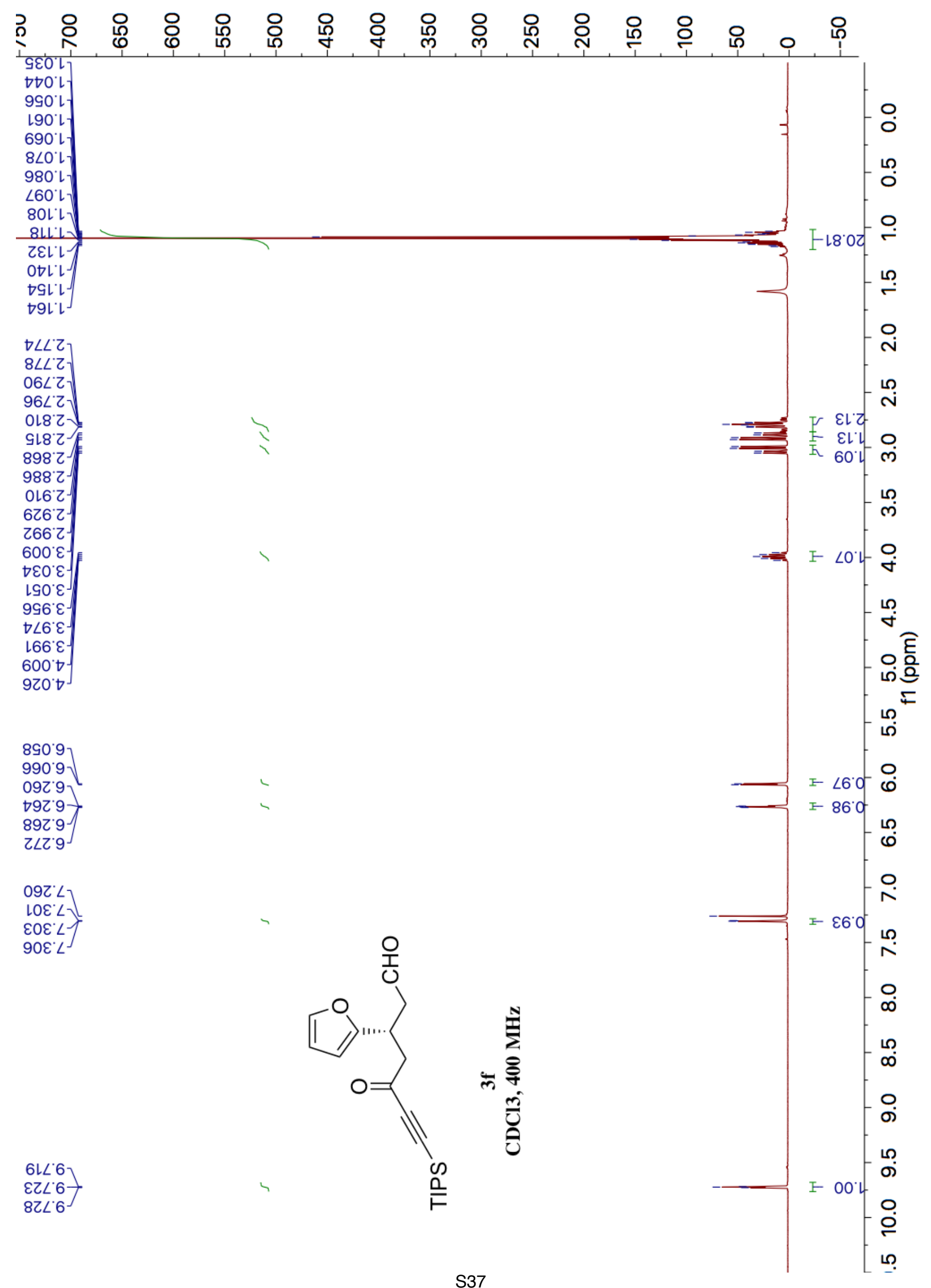




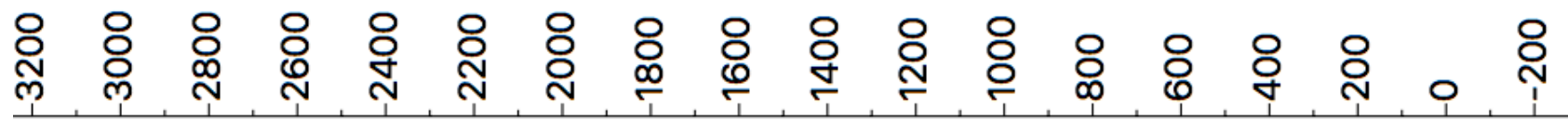

t๑6.0L-

ost 81

$\neg \varepsilon 062-$

$679 \cdot 9 t>$

$\angle 699^{\circ} \mathrm{t}-$

$6 เ \varepsilon: L-$

026.96

$\varepsilon 8 L^{\circ} \varepsilon \mathrm{OL}$ ᄀ

$888^{\circ} \mathrm{GOL}-$

StZ'OlL

†Lடレレー

Z88 $\bullet 9 L-$

เ89เ8เ-

6ll'002-

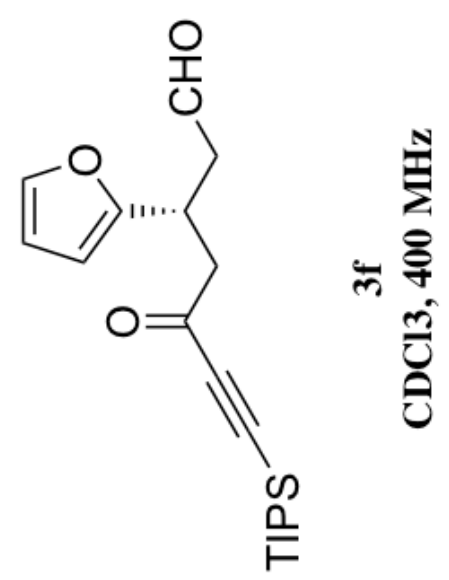




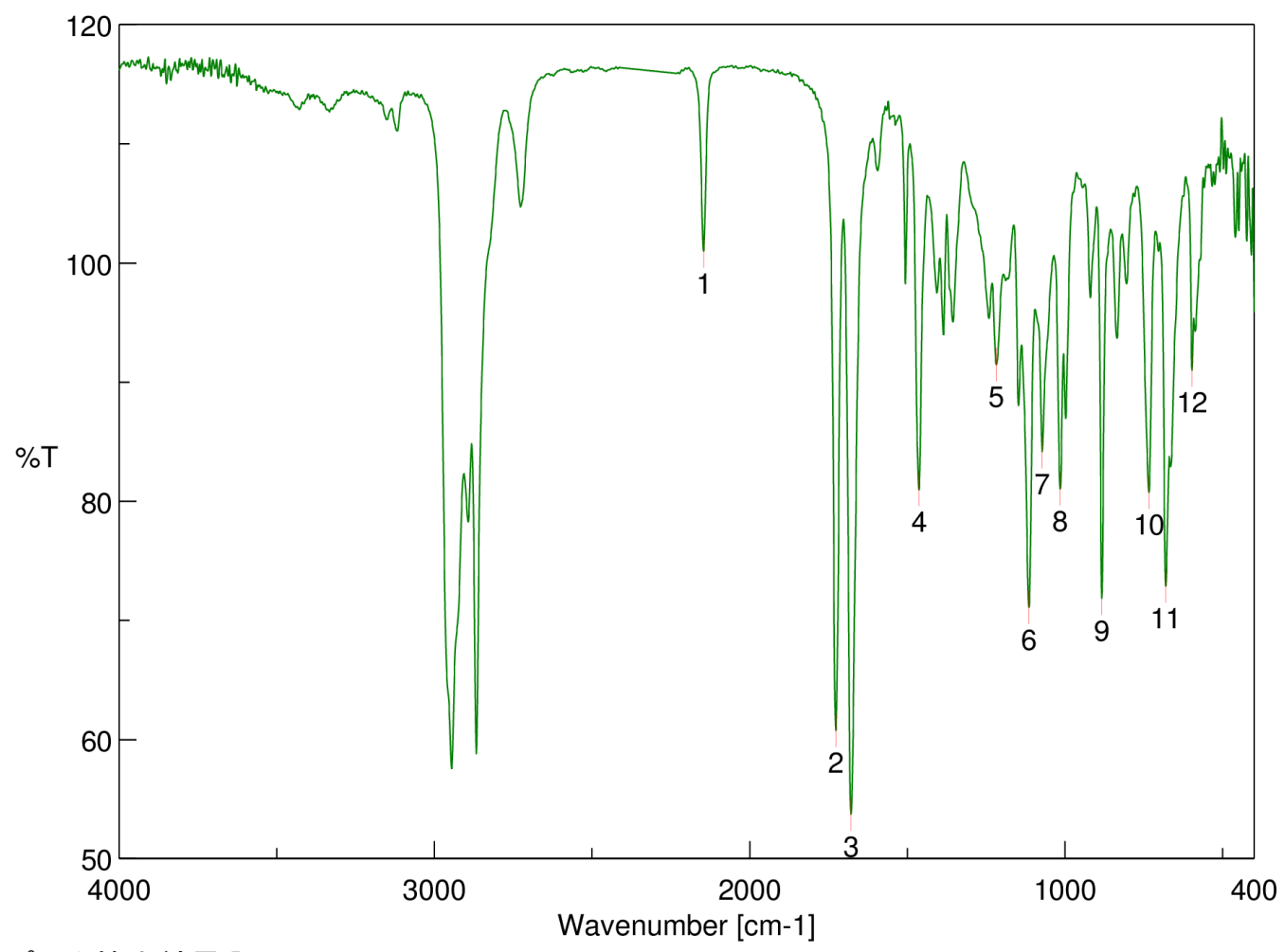

[ピーク検出結果 ]

$\begin{array}{llllll}\text { No. } & \text { 位置 } & \text { 強度 } & \text { No. } & \text { 位置 } & \text { 強度 } \\ 1 & 2146.38 & 100.971 & 2 & 1726.94 & 60.748 \\ 3 & 1678.73 & 53.7382 & 4 & 1463.71 & 80.9579 \\ 5 & 1217.83 & 91.4577 & 6 & 1114.65 & 71.0891 \\ 7 & 1072.23 & 84.1533 & 8 & 1015.34 & 81.0283 \\ 9 & 883.238 & 71.8622 & 10 & 733.782 & 80.7281 \\ 11 & 680.749 & 72.8752 & 12 & 596.861 & 90.9865\end{array}$<smiles>O=CC[C@H](CC(=O)C#CSP)c1ccco1</smiles> 


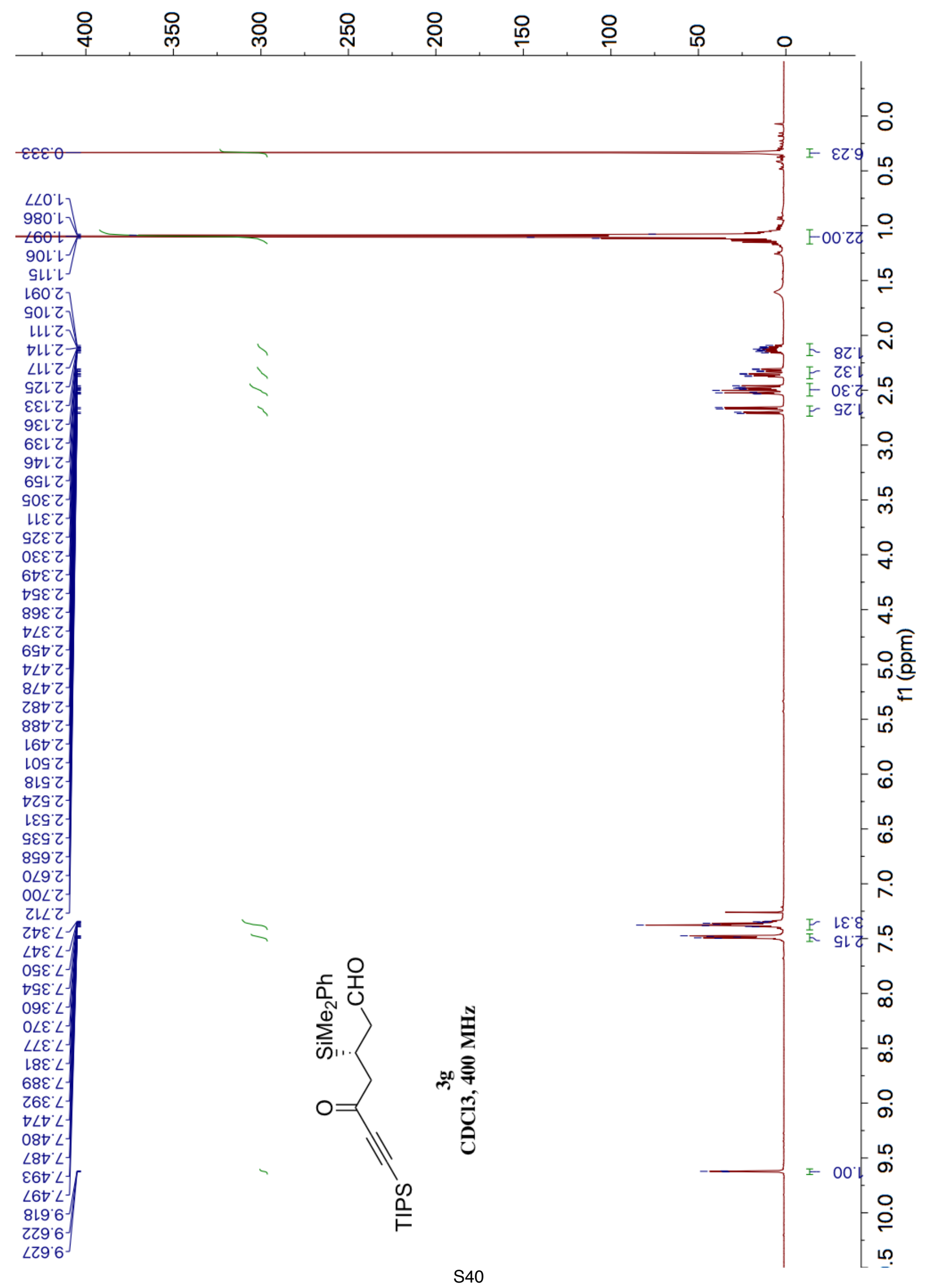


然员

$\varepsilon 9 \nabla^{*} \nabla^{-}$
$99 \varepsilon^{\circ} \nabla^{-}$

G96.OL-

ยてォ'งレ

29t'8l-

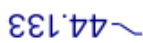

8L6'Gt

乙89. 94

$000 \angle L \frac{1}{5}$

$6 L E^{\prime} L L$

$\angle 8 \varepsilon^{\circ} 96-$

8LO`OL-

9Z0.8ZL

0t9.6ZL-

\&L8'E\&L-

$\angle \varepsilon Z^{\prime} 9 \varepsilon L^{-}$

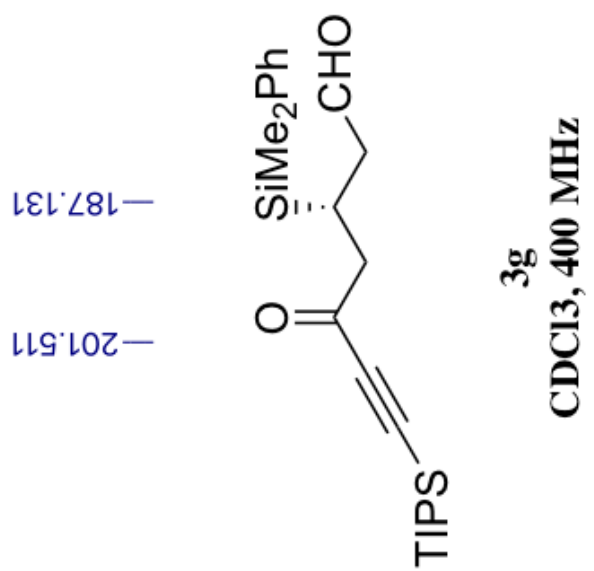




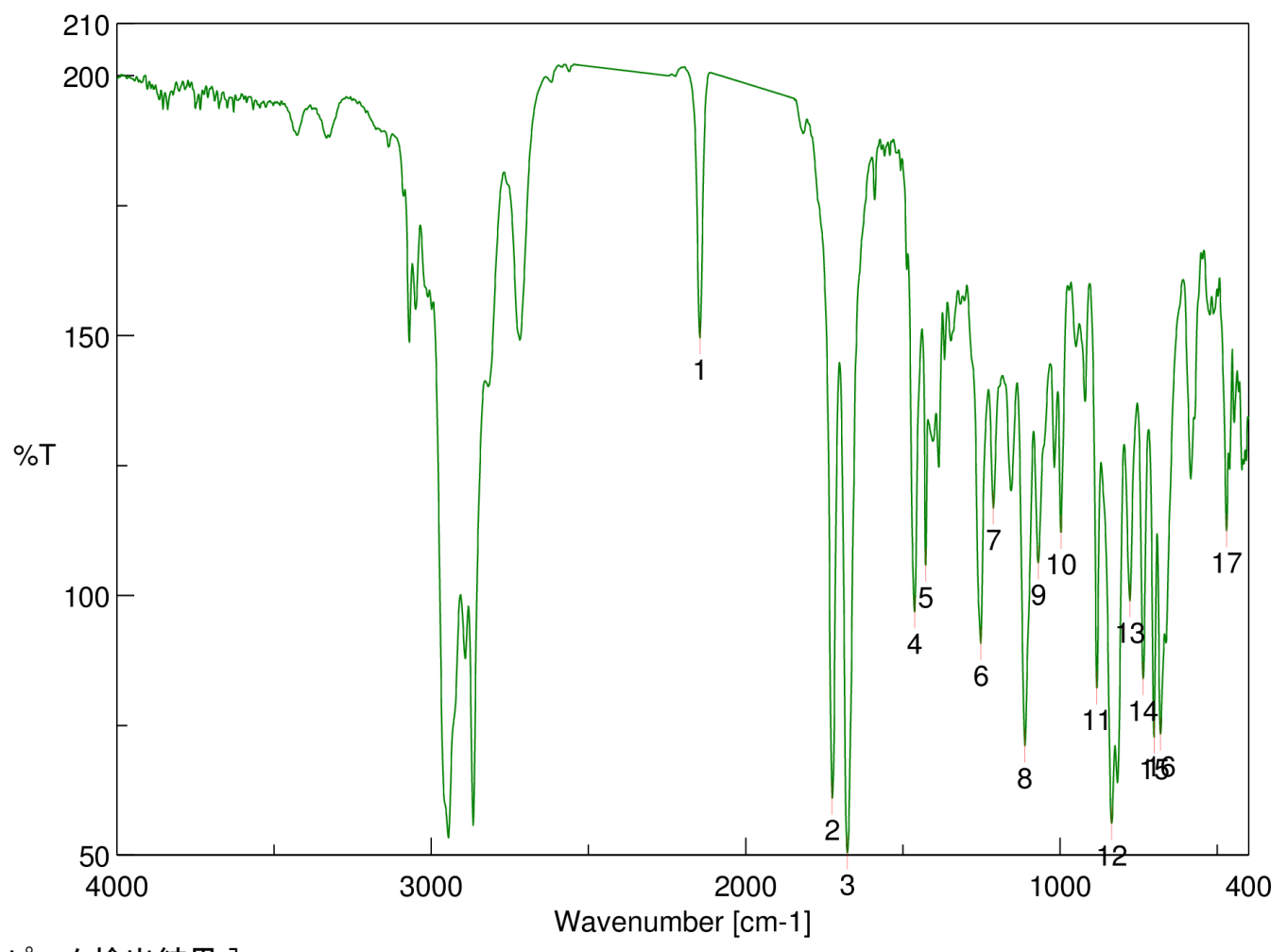

\section{[ピーク検出結果 ]}

$\begin{array}{llllll}\text { No. } & \text { 位置 } & \text { 強度 } & \text { No. } & \text { 位置 } & \text { 強度 } \\ 1 & 2145.42 & 149.562 & 2 & 1725.01 & 60.9598 \\ 3 & 1676.8 & 50.4834 & 4 & 1462.74 & 96.8409 \\ 5 & 1427.07 & 105.773 & 6 & 1252.54 & 90.7543 \\ 7 & 1212.04 & 116.774 & 8 & 1111.76 & 71.0225 \\ 9 & 1069.33 & 106.275 & 10 & 997.017 & 112.131 \\ 11 & 883.238 & 82.1869 & 12 & 835.99 & 56.1477 \\ 13 & 778.136 & 98.9593 & 14 & 735.71 & 83.9728 \\ 15 & 700.998 & 72.7094 & 16 & 680.749 & 73.2955 \\ 17 & 470.546 & 112.448 & & & \end{array}$

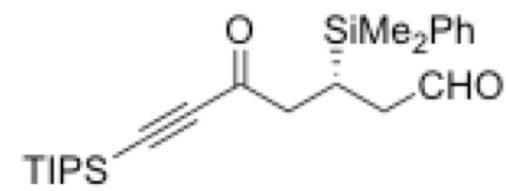




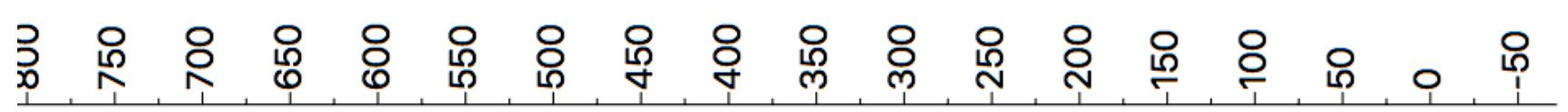

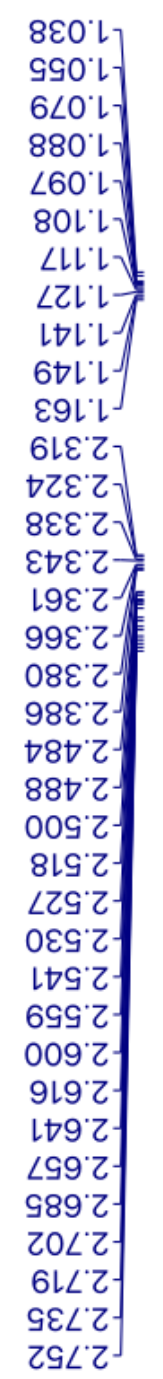

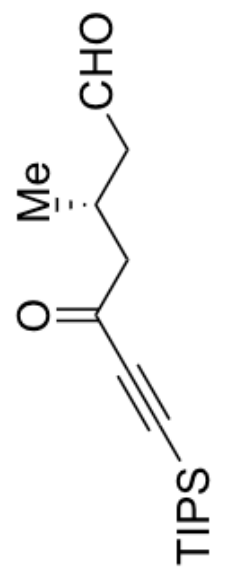

ल 


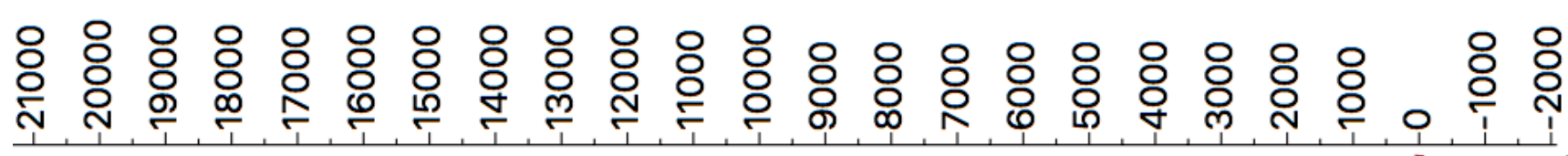

$2 \angle 60^{\circ} \mathrm{L}-$

\&9t $8 \mathrm{~L}$

$\angle 00^{\circ} \mathrm{OZ}-$

แI9 $\downarrow Z$ -

$610.09-$

6L8'LG

$189^{\circ} 9 \angle$

\begin{tabular}{l|l|}
$-\frac{0}{1}$ \\
0 \\
0
\end{tabular}

ont

$8 \mathrm{LE} \angle L$

ع6เ'96-

๖乙ঁヤOL-

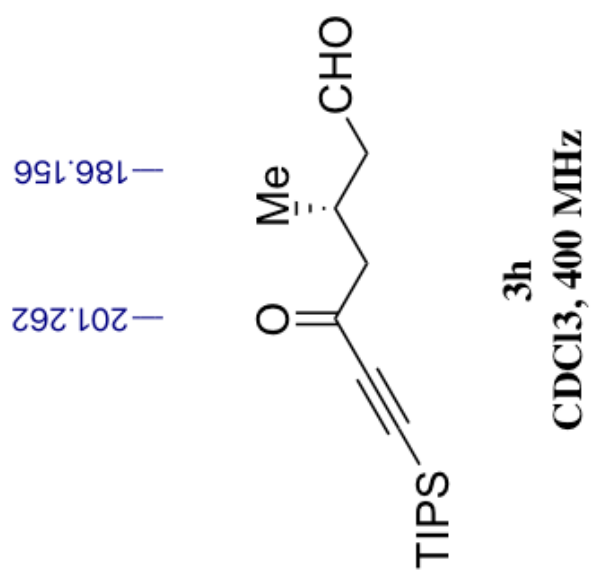




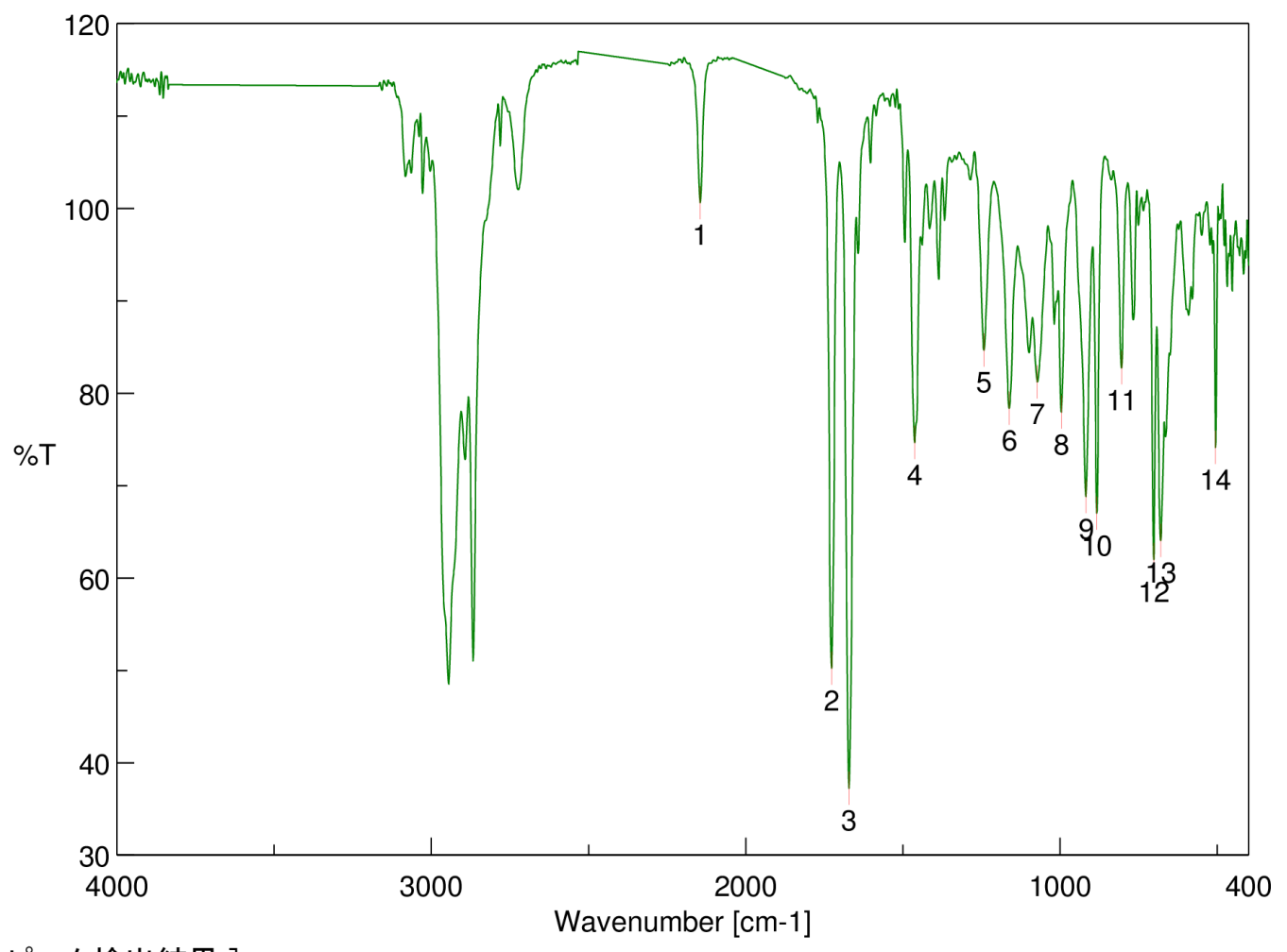

[ピーク検出結果 ]

$\begin{array}{llllll}\text { No. } & \text { 位置 } & \text { 強度 } & \text { No. } & \text { 位置 } & \text { 強度 } \\ 1 & 2144.46 & 100.583 & 2 & 1726.94 & 50.2365 \\ 3 & 1671.02 & 37.2193 & 4 & 1462.74 & 74.643 \\ 5 & 1241.93 & 84.6359 & 6 & 1161.9 & 78.3419 \\ 7 & 1072.23 & 81.215 & 8 & 996.053 & 77.9284 \\ 9 & 917.95 & 68.8086 & 10 & 883.238 & 67.0089 \\ 11 & 804.171 & 82.7392 & 12 & 701.962 & 61.976 \\ 13 & 679.785 & 64.0461 & 14 & 505.258 & 74.1117\end{array}$<smiles>CC(CC=O)CC(=O)C#C[AsH]</smiles> 


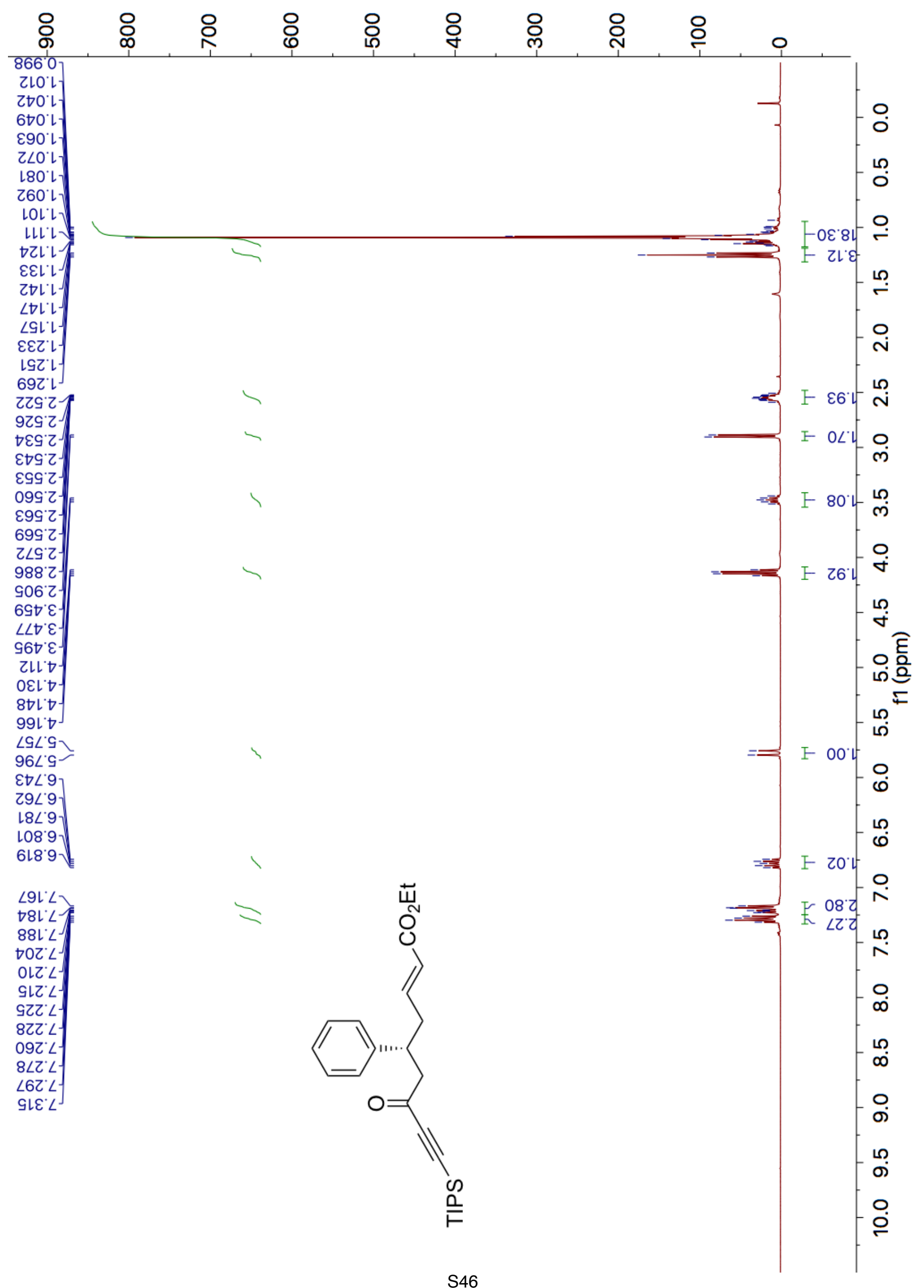


임 이 \&

$2960^{\circ} \mathrm{OL}-$

Z6เけレ-

L $\angle \nabla ' 8 L$

Z19.8E

s99.0t

967เS-

902 09-

$189^{\circ} 9 /$

$000 \angle L J$

$8 \mathrm{~L} \varepsilon^{\prime} \angle L$

เ9t96-

カtレナOLー

${ }^{\circ} \mathrm{G}^{\circ} \varepsilon Z \mathrm{~L}$

$696^{\circ} 9 \mathrm{ZL}$

$\angle S E^{\circ} \angle Z L Y$

ZEL $8 Z L$

เ9でてレー

เย9'งฺレ-

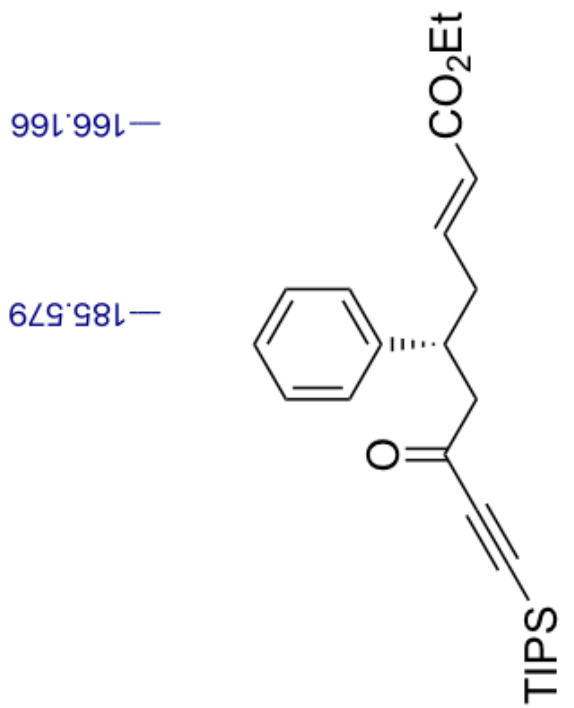




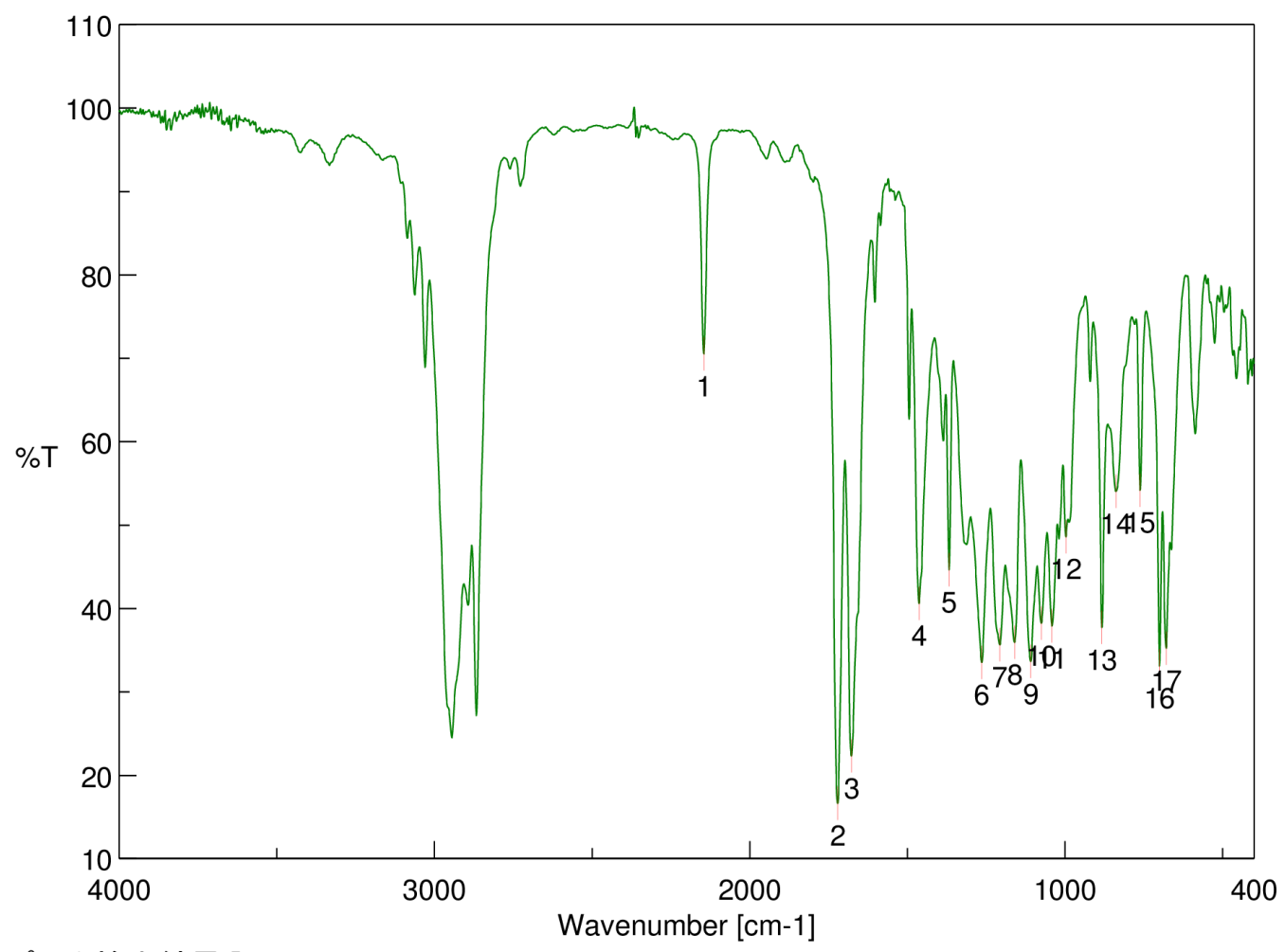

[ピーク検出結果 ]

$\begin{array}{llllll}\text { No. } & \text { 位置 } & \text { 強度 } & \text { No. } & \text { 位置 } & \text { 強度 } \\ 1 & 2145.42 & 70.4999 & 2 & 1721.16 & 16.6109 \\ 3 & 1676.8 & 22.3269 & 4 & 1462.74 & 40.5704 \\ 5 & 1367.28 & 44.627 & 6 & 1264.11 & 33.5003 \\ 7 & 1207.22 & 35.6323 & 8 & 1159.97 & 35.9157 \\ 9 & 1108.87 & 33.6187 & 10 & 1075.12 & 38.2352 \\ 11 & 1041.37 & 37.8997 & 12 & 997.017 & 48.5932 \\ 13 & 883.238 & 37.7165 & 14 & 837.919 & 54.0177 \\ 15 & 761.744 & 54.1649 & 16 & 700.034 & 33.1012 \\ 17 & 678.82 & 35.2257 & & & \end{array}$<smiles>CCOC(=O)/C=C/C[C@H](CC(=O)C#CS[In])c1ccccc1</smiles> 


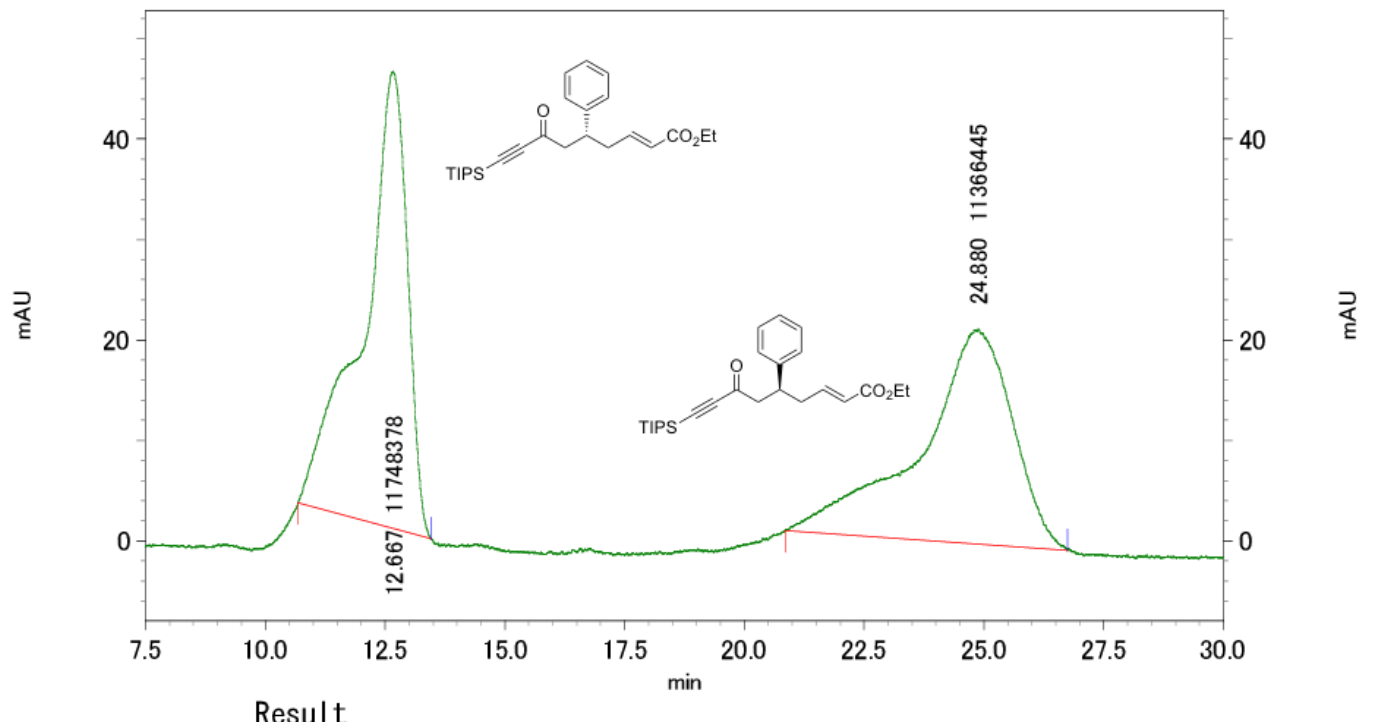

1: $238 \mathrm{~nm}, 4 \mathrm{~nm}$ 結果

\begin{tabular}{|r|r|}
\hline$r-$ 㚈 & 100.000 \\
\hline
\end{tabular}




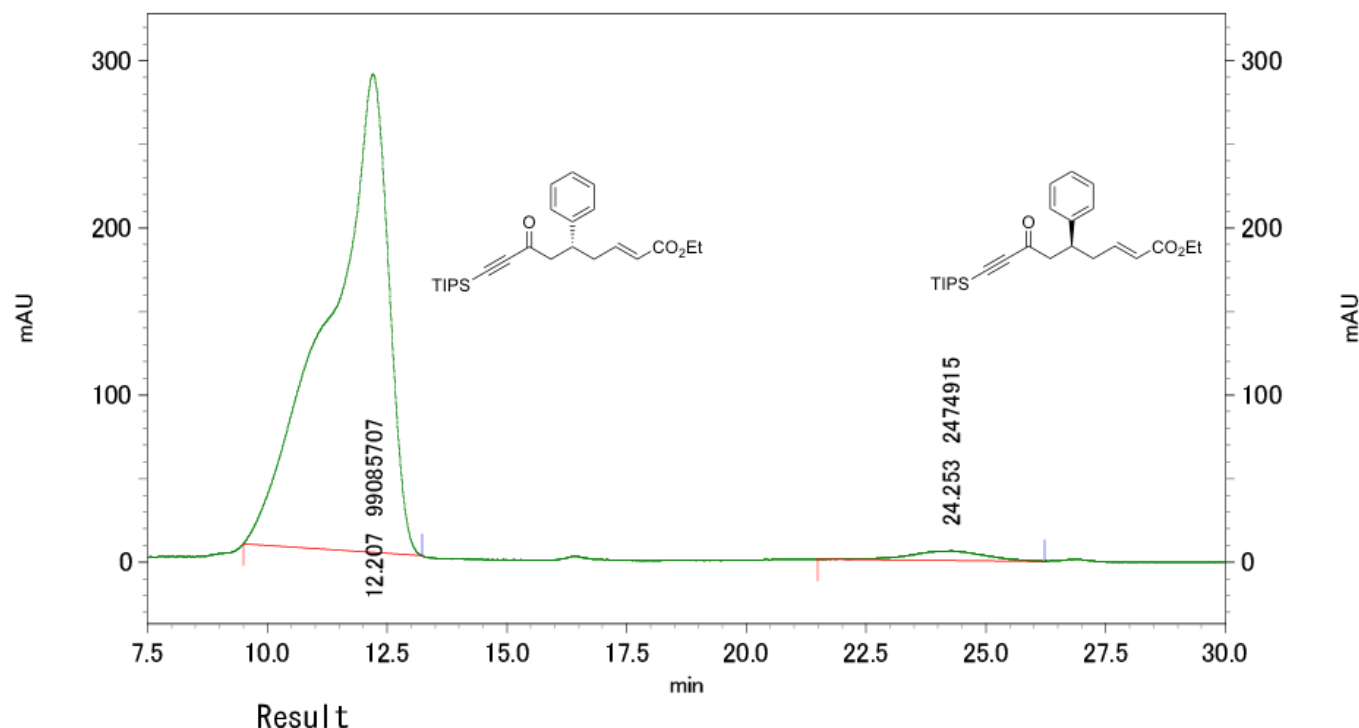

1: $238 \mathrm{~nm}, 4$ nm結果

Integration/ \%

\begin{tabular}{|r|r|r|}
\hline$r-$ 舛 & 100.000 \\
\hline
\end{tabular}




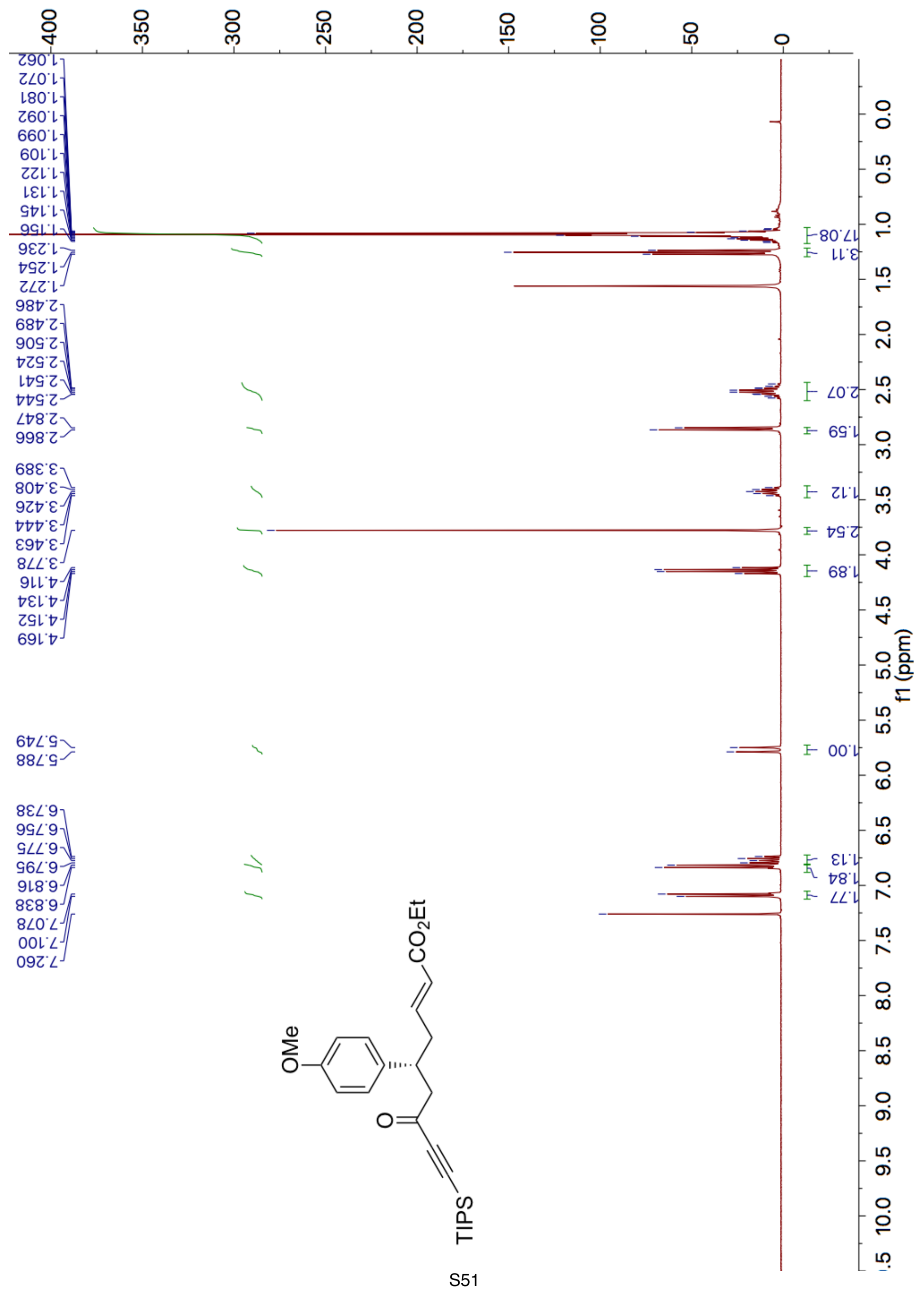




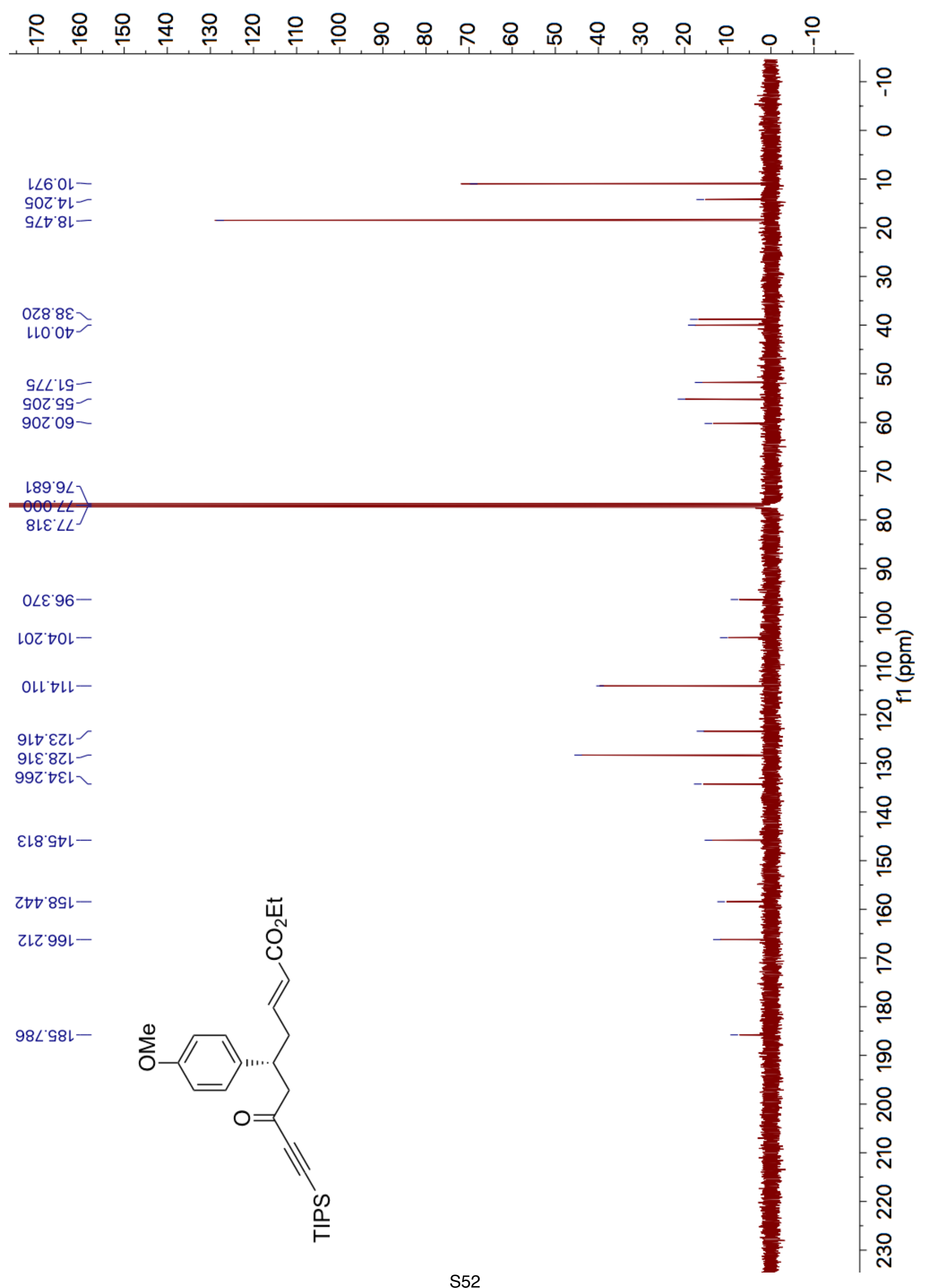




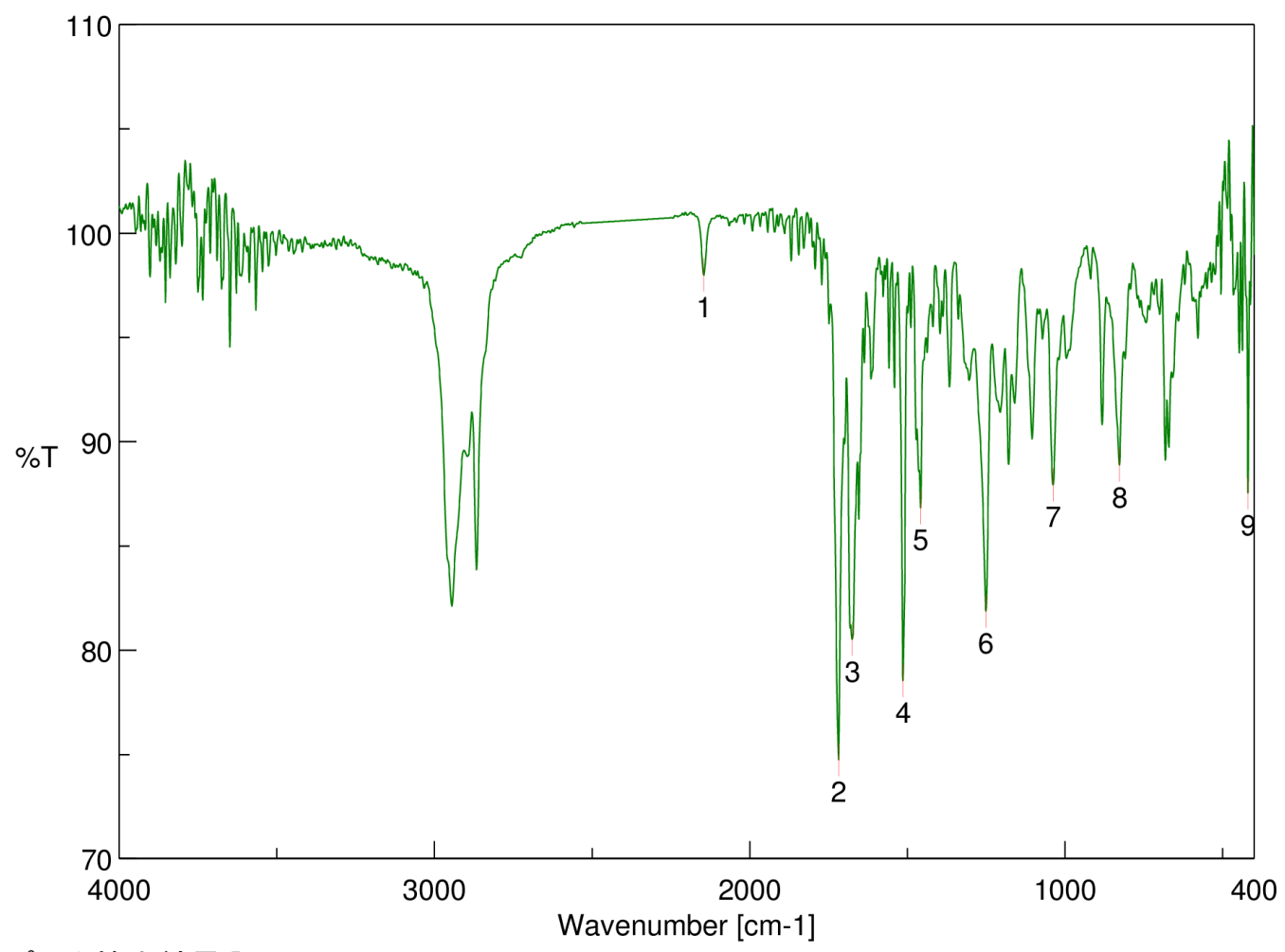

[ピーク検出結果 ]

$\begin{array}{llllll}\text { No. } & \text { 位置 } & \text { 強度 } & \text { No. } & \text { 位置 } & \text { 強度 } \\ 1 & 2146.38 & 97.9915 & 2 & 1718.26 & 74.7363 \\ 3 & 1674.87 & 80.5174 & 4 & 1513.85 & 78.543 \\ 5 & 1457.92 & 86.8269 & 6 & 1250.61 & 81.8696 \\ 7 & 1037.52 & 87.923 & 8 & 827.312 & 88.8837 \\ 9 & 419.442 & 87.5454 & & & \end{array}$<smiles>CC#CC(=O)CC(CCCC(=O)OCC)c1ccc(OC)cc1</smiles> 


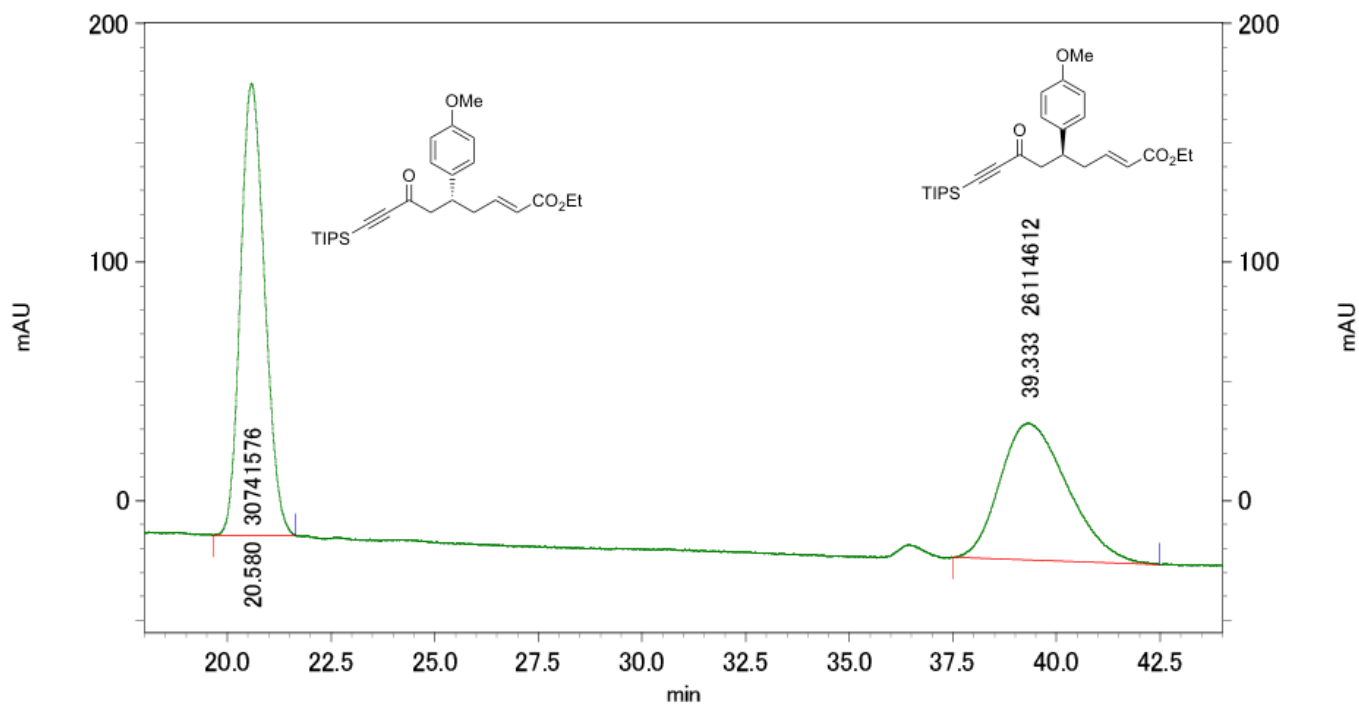

Result

1: $213 \mathrm{~nm}, 4 \mathrm{~nm}$ 結果

Pk \# Retention time / min

20. 380

Integration/\%

45.931

\begin{tabular}{|r|r|r|}
\hline$r-$ gll & 100.000 \\
\hline
\end{tabular} 


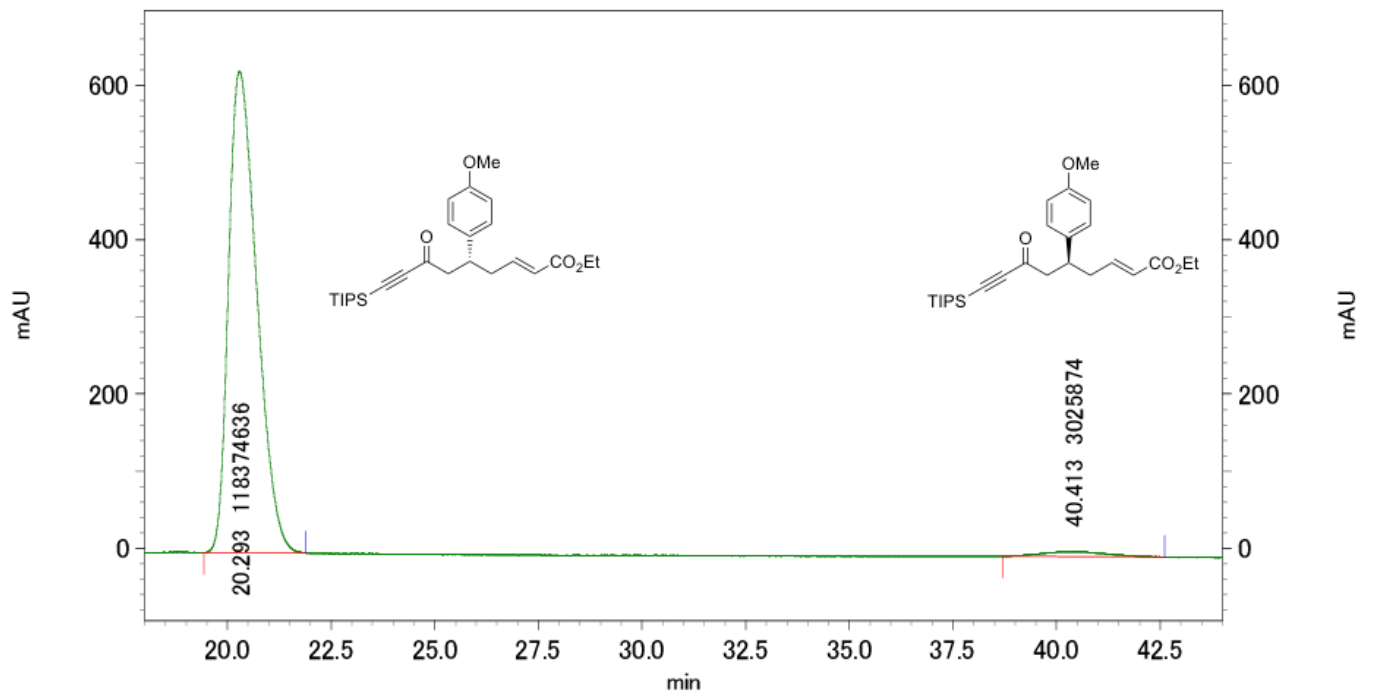

Result

1: $213 \mathrm{~nm}, 4 \mathrm{~nm}$ 結果

Pk \# Retention time / min

20.293
40.413

Integration/\%

2. 492

\begin{tabular}{|r|r|}
\hline r-夕夕l & 100.000 \\
\hline Total
\end{tabular} 


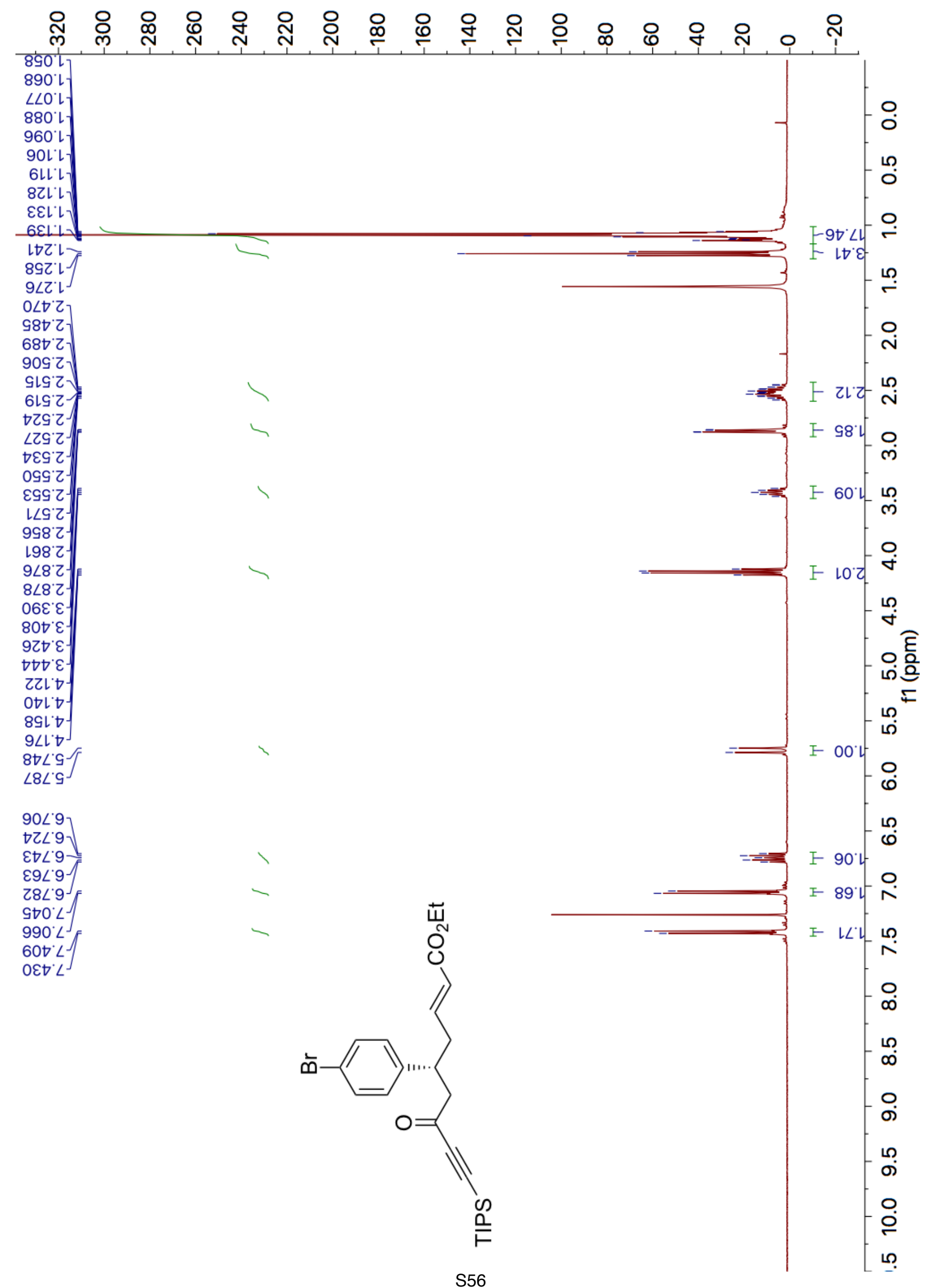




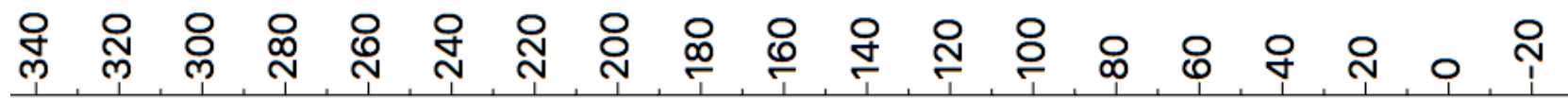

996.OL-

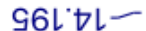

†97.8L-

9乙† $8 \varepsilon-$

Z6l'Ot

Z9Zเง-

oเع.09-

乙ะ8'96-

$\checkmark \angle 0^{\circ} \nabla O L-$

$008^{\circ} 0 Z L-$

608'धZL-

0El'6Zl

698'LEL-

てャでレレー

9ع0’G๋L-

L90.99L-

ยฑเ'G8เ-

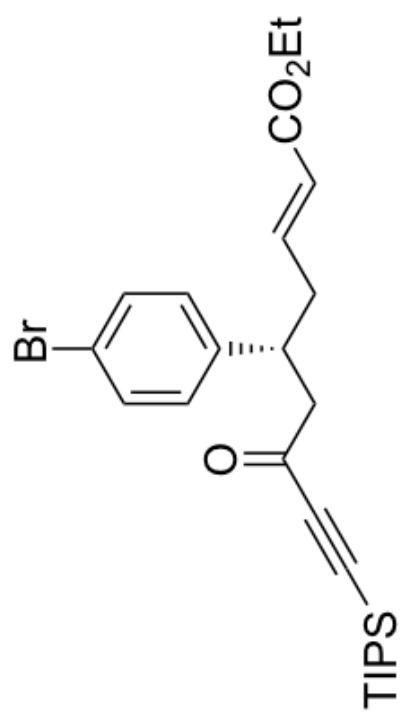




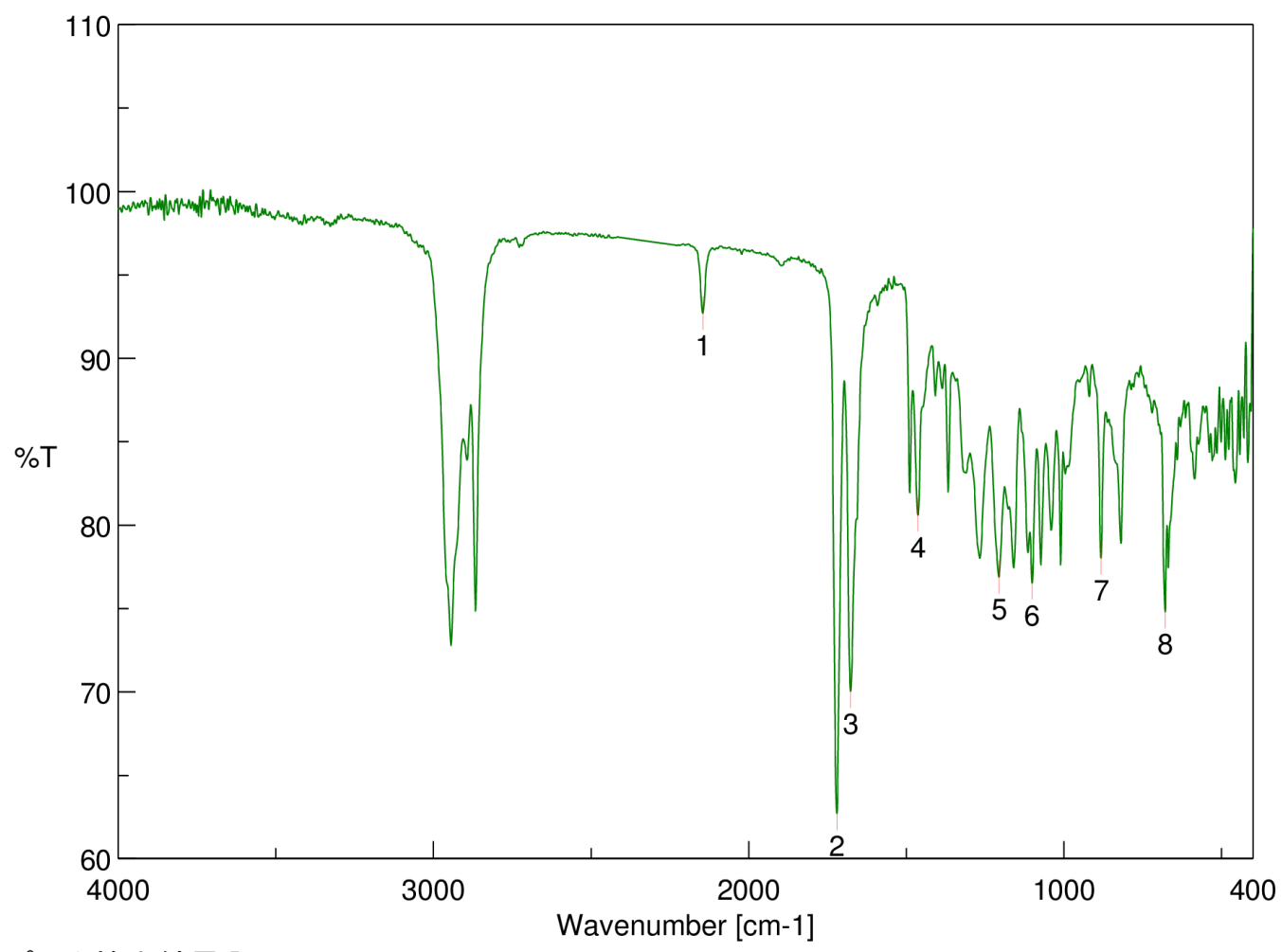

[ピーク検出結果 ]

$\begin{array}{llllll}\text { No. } & \text { 位置 } & \text { 強度 } & \text { No. } & \text { 位置 } & \text { 強度 } \\ 1 & 2145.42 & 92.6808 & 2 & 1720.19 & 62.7037 \\ 3 & 1676.8 & 70.0386 & 4 & 1463.71 & 80.5901 \\ 5 & 1206.26 & 76.8825 & 6 & 1101.15 & 76.5214 \\ 7 & 882.274 & 78.0259 & 8 & 678.82 & 74.7902\end{array}$

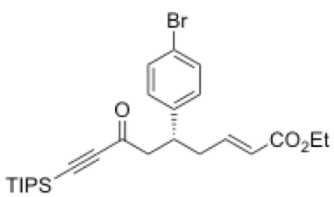




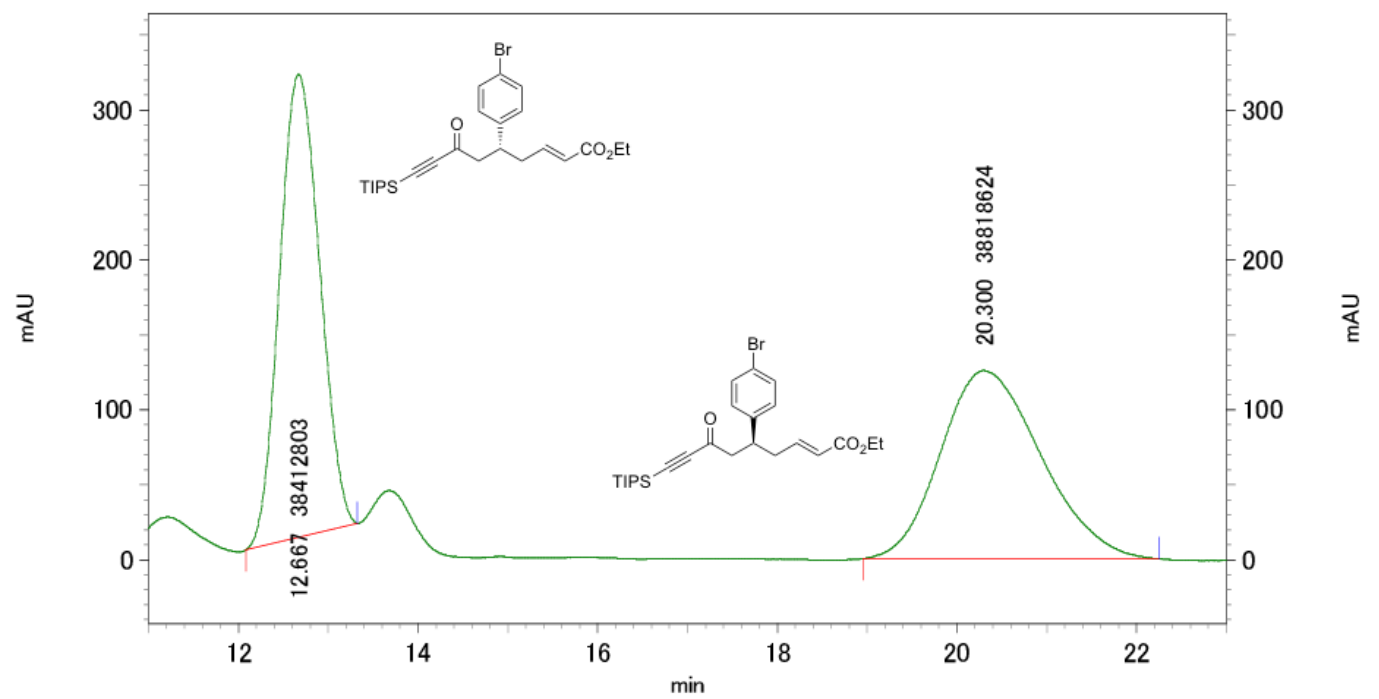

Result

1: $234 \mathrm{~nm}, 4$ nm結果

\begin{tabular}{|r|r|r|}
\hline$r-$ 多 & 100.000 \\
\hline
\end{tabular}




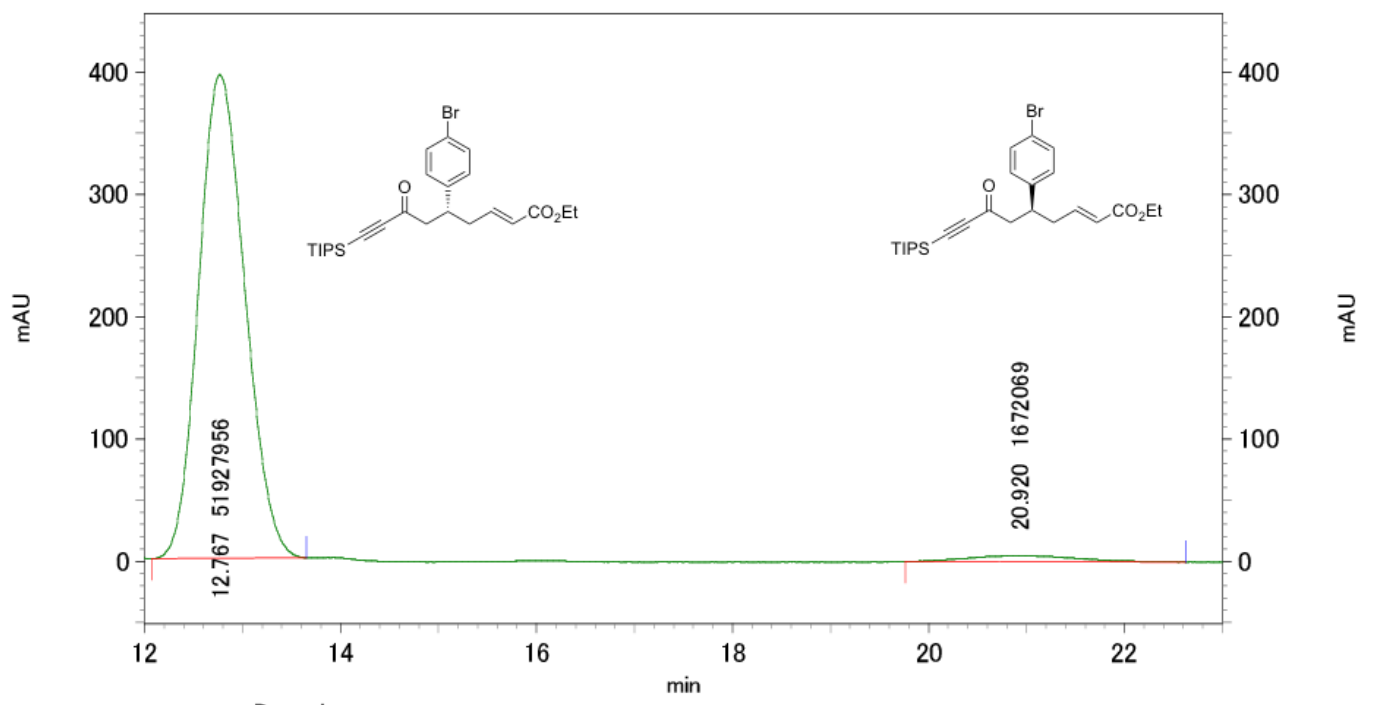

1: $233 \mathrm{~nm}, 4 \mathrm{~nm}$ 結果

Pk \# Retention time / min

12.767
20.920

Integration/\%

3. 120

\begin{tabular}{|r|r|}
\hline$r-$ 㚈 & 100.000 \\
\hline
\end{tabular} 


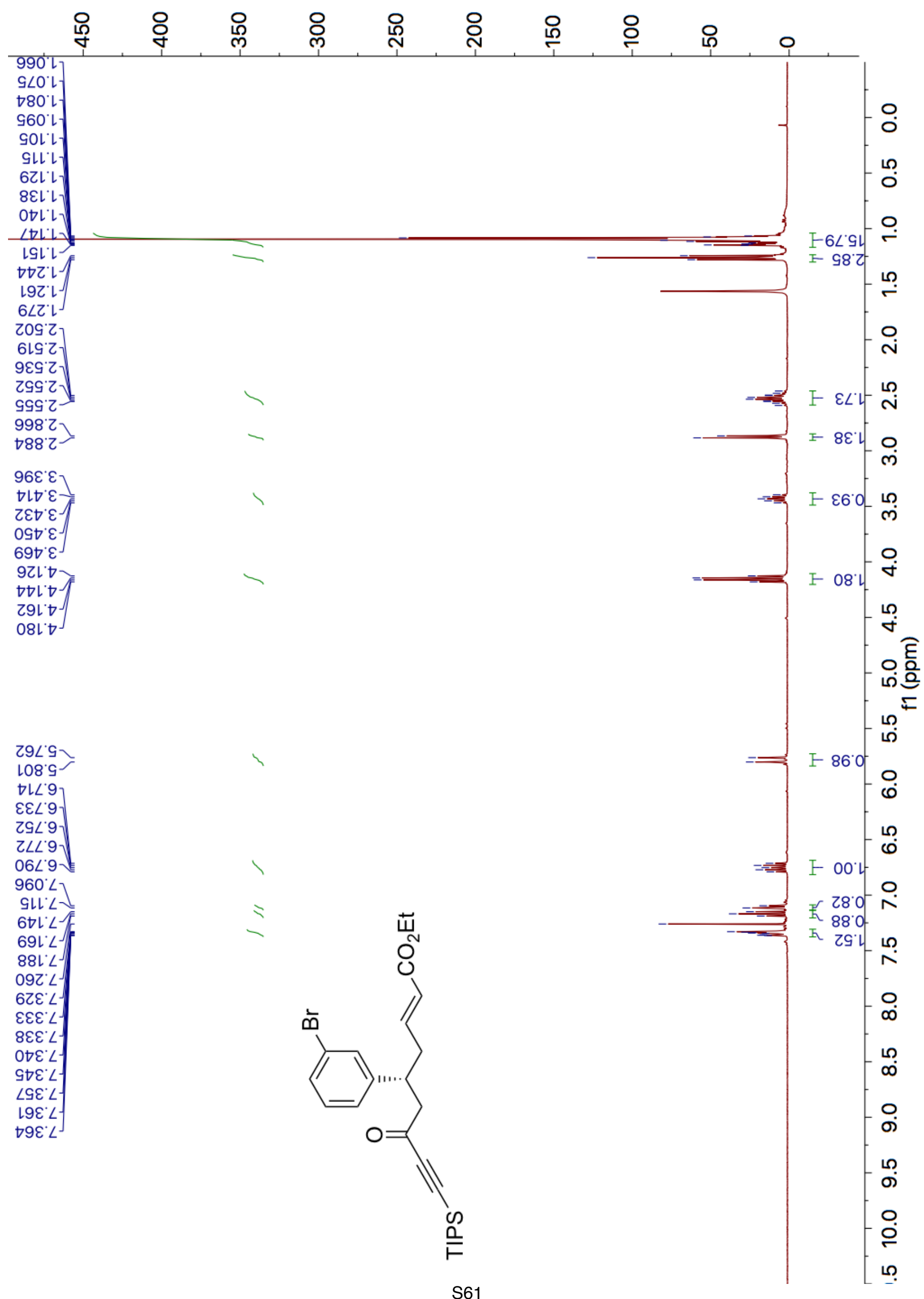


$6 L t^{\circ} 8 \varepsilon-$

$9 เ \varepsilon^{\circ} 0 t^{-}$

9งเ'เง-

ZเE'09-

$289^{\circ} 9<$

$000 \angle L T$

$6 L \varepsilon \angle L$

906 $96-$

$8 Z 0^{\circ} \circ 0 \mathrm{~L}-$

เ๑8 ててレ

$898^{\circ} \mathrm{EL}$.
I9l'9ZL

ZOZ OEL

$60 \varepsilon^{\circ} 0 \varepsilon L_{-}$

lZ† $0 \varepsilon \downarrow$

989 t十t

$\nabla 96 \bullet t \downarrow$

Z90.991-

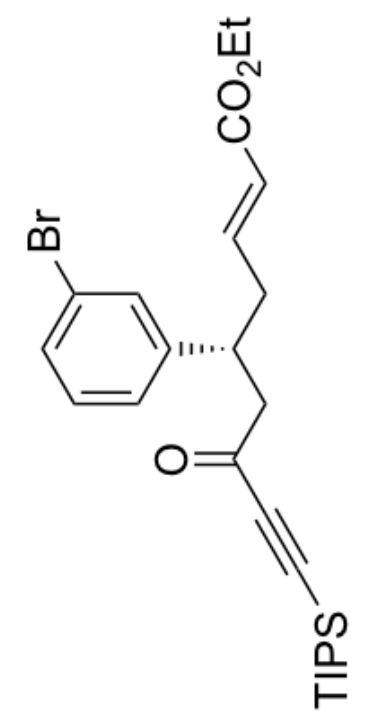




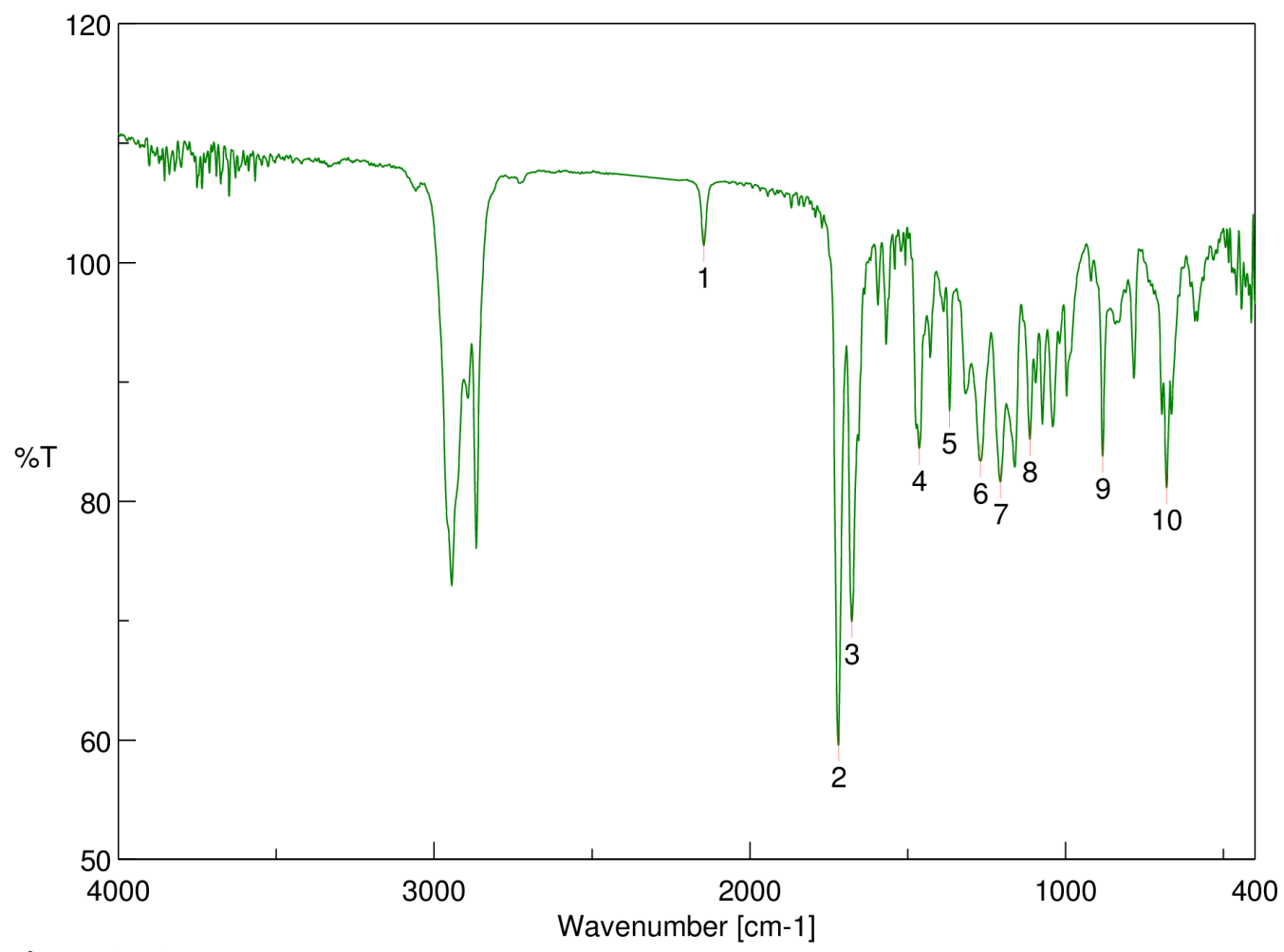

[ピーク検出結果 ]

$\begin{array}{llllll}\text { No. } & \text { 位置 } & \text { 強度 } & \text { No. } & \text { 位置 } & \text { 強度 } \\ 1 & 2146.38 & 101.433 & 2 & 1719.23 & 59.5812 \\ 3 & 1676.8 & 69.9177 & 4 & 1463.71 & 84.4217 \\ 5 & 1367.28 & 87.5675 & 6 & 1269.9 & 83.3779 \\ 7 & 1206.26 & 81.6407 & 8 & 1112.73 & 85.1871 \\ 9 & 882.274 & 83.7914 & 10 & 679.785 & 81.126\end{array}$<smiles>CCCCC#CC(=O)CC(CCCC(=O)OCC)c1cccc(Br)c1</smiles> 


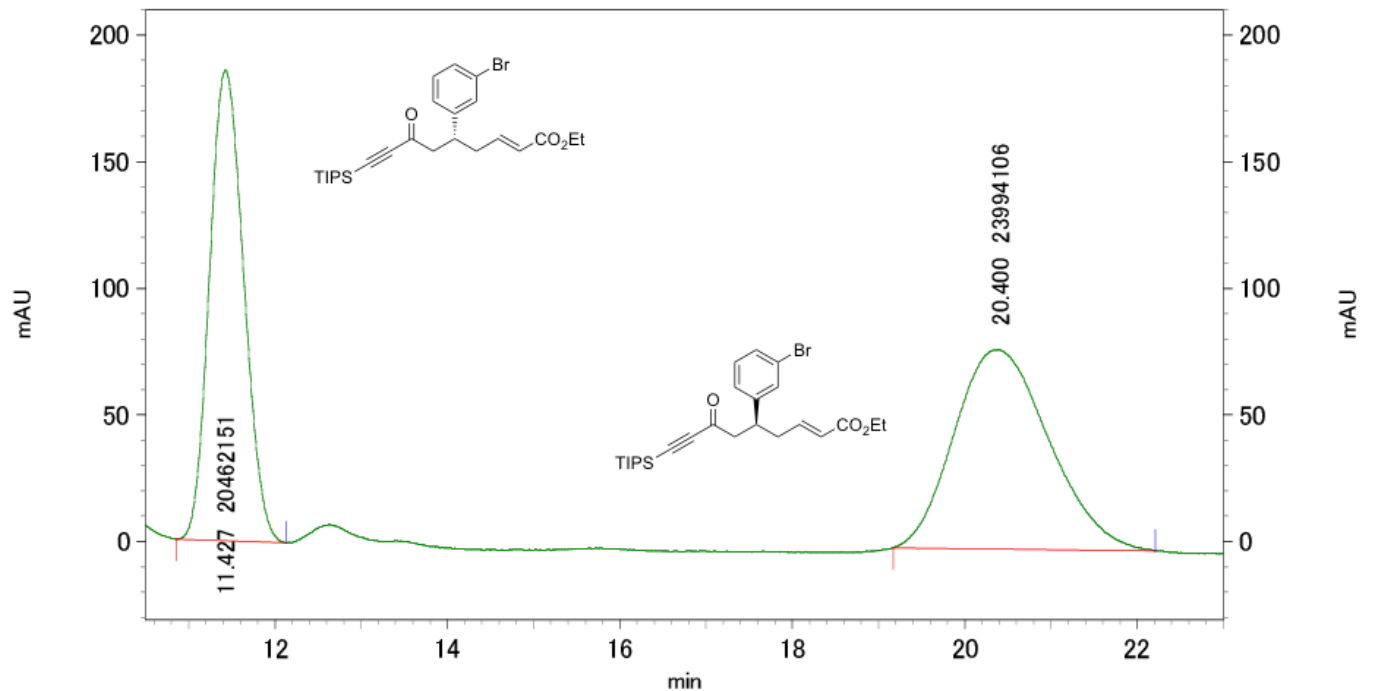

Result

1: $234 \mathrm{~nm}, 4$ nm結果

\begin{tabular}{|r|r|r|}
\hline$r-$ 多 & 100.000 \\
\hline
\end{tabular}




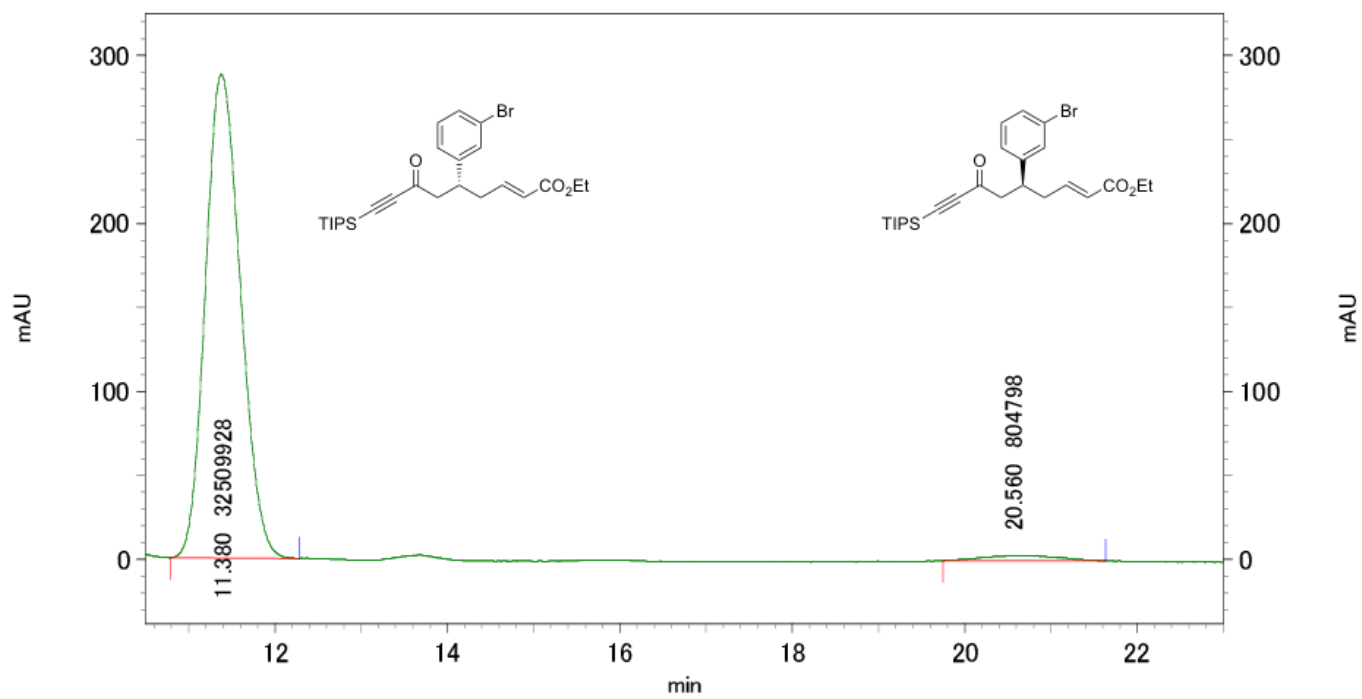

1: $234 \mathrm{~nm}, 4$ nm結果

Pk \# Retention time / min

11.380
20.560

$\frac{\text { Integration/\% }}{97.584}$

2. 416

\begin{tabular}{|r|r|}
\hline$r-$ 㚈 & 100.000 \\
\hline
\end{tabular} 


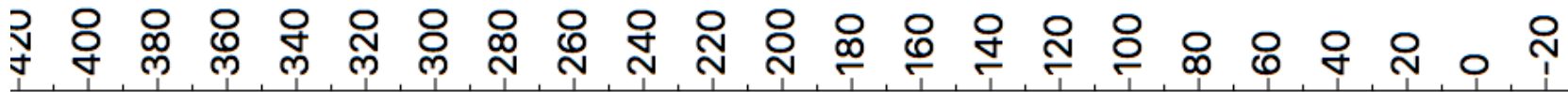

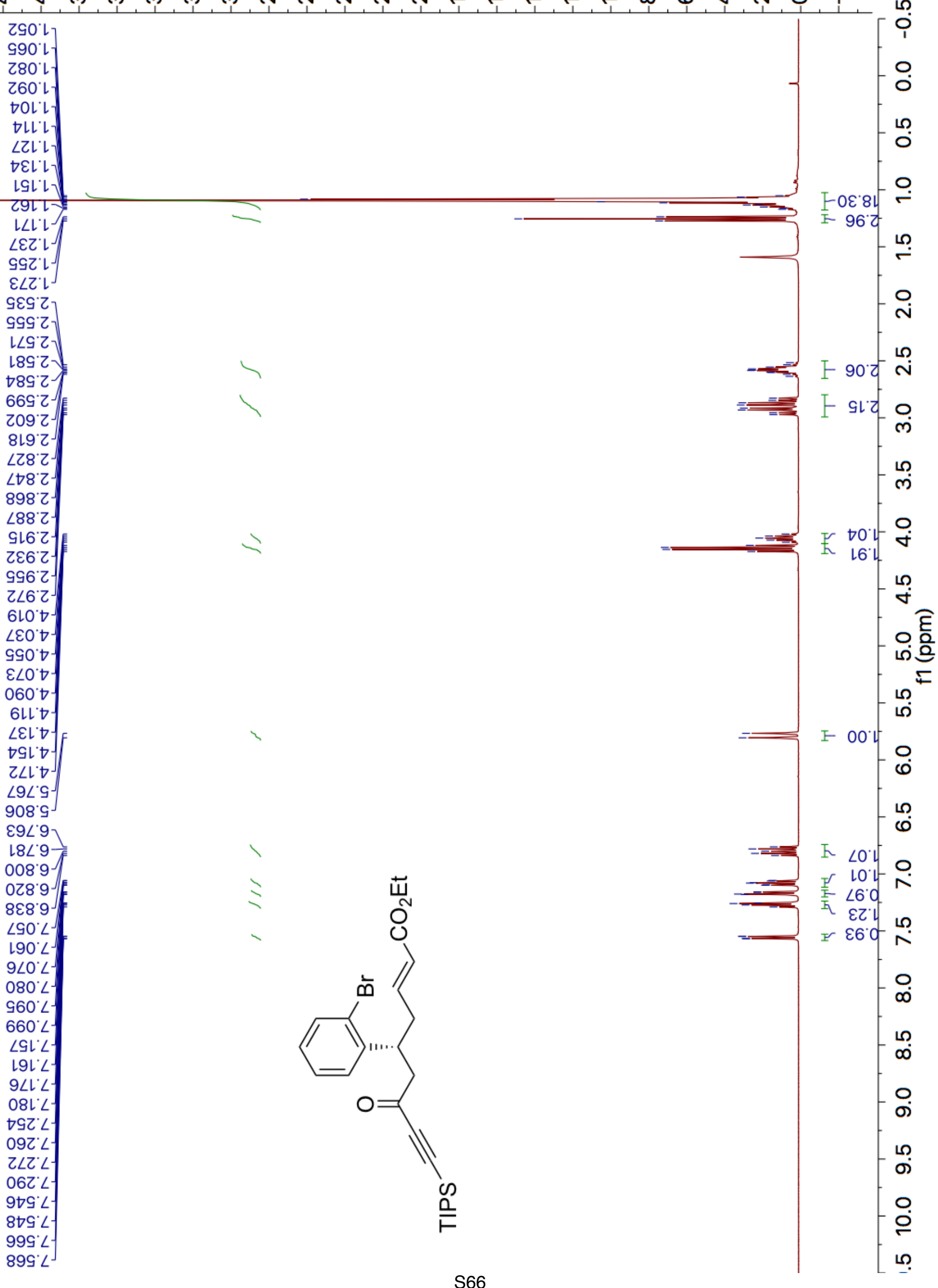




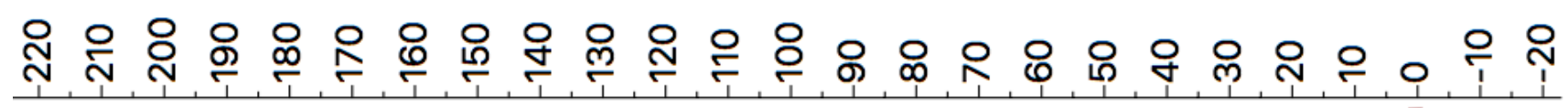

SL6'OL-

Z6L'th-

$68 t^{\circ} 8 \mathrm{~L}$

$\angle \varepsilon 0^{\circ} \angle \mathcal{E}-$

Z86 $8 \varepsilon^{\circ}$

$87867-$

LEZ 09-

189.94

$000 \angle L T$

$8 L \varepsilon^{\circ} L L$

IZL'96-

G68' $\mathrm{EOL-}$

Sเ8'EZL

$0 G \angle \backslash \nabla Z L$

$\forall L L \angle Z L-$

998 $8 \mathrm{Ll}^{\circ}$

$\angle 6 \varepsilon \cdot \varepsilon \varepsilon \mathrm{L}$

LZ6.0tL

$\nabla 00$ Gt

$880^{\circ} 991-$

งZเ'98เ-

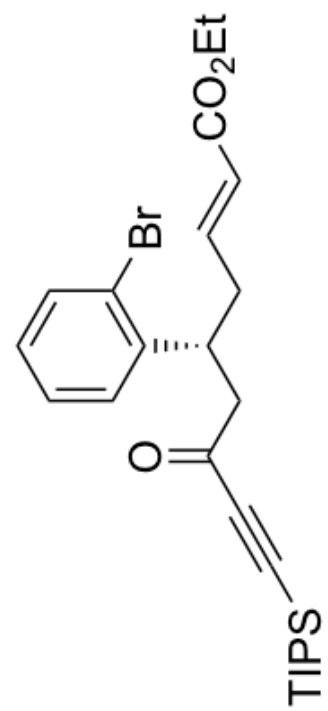




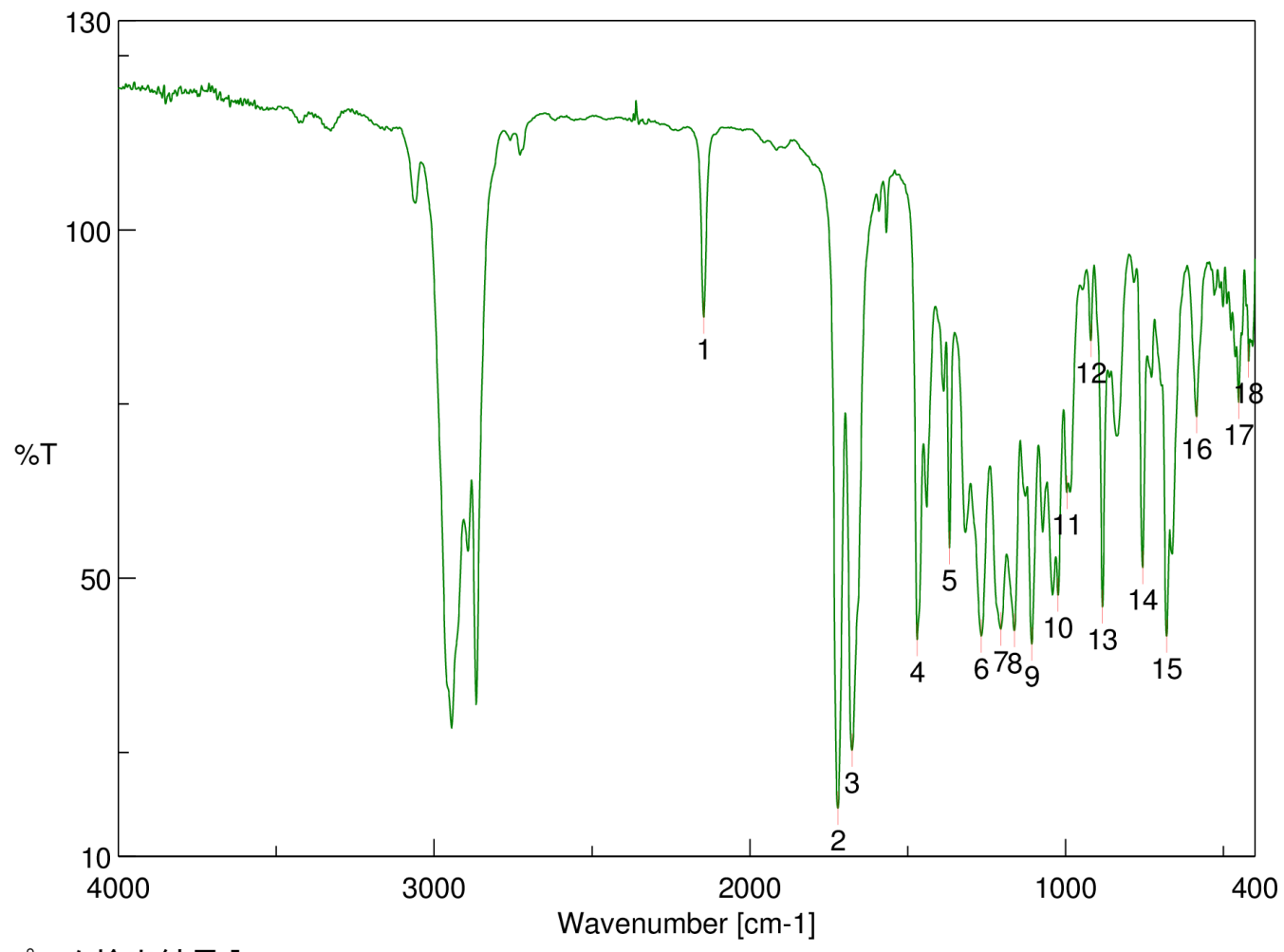

\section{[ピーク検出結果 ]}

$\begin{array}{llllll}\text { No. } & \text { 位置 } & \text { 強度 } & \text { No. } & \text { 位置 } & \text { 強度 } \\ 1 & 2146.38 & 87.4447 & 2 & 1721.16 & 16.9312 \\ 3 & 1675.84 & 25.2578 & 4 & 1470.46 & 41.1771 \\ 5 & 1367.28 & 54.3629 & 6 & 1267 & 41.6697 \\ 7 & 1205.29 & 42.6426 & 8 & 1161.9 & 42.4444 \\ 9 & 1106.94 & 40.5302 & 10 & 1024.02 & 47.5424 \\ 11 & 996.053 & 62.2804 & 12 & 919.879 & 84.0874 \\ 13 & 883.238 & 45.8727 & 14 & 755.959 & 51.4923 \\ 15 & 679.785 & 41.676 & 16 & 585.29 & 73.1495 \\ 17 & 452.225 & 75.2175 & 18 & 420.406 & 81.1371\end{array}$<smiles>CCOC=CCC(CC(=O)C#CC#N)c1ccccc1Br</smiles> 


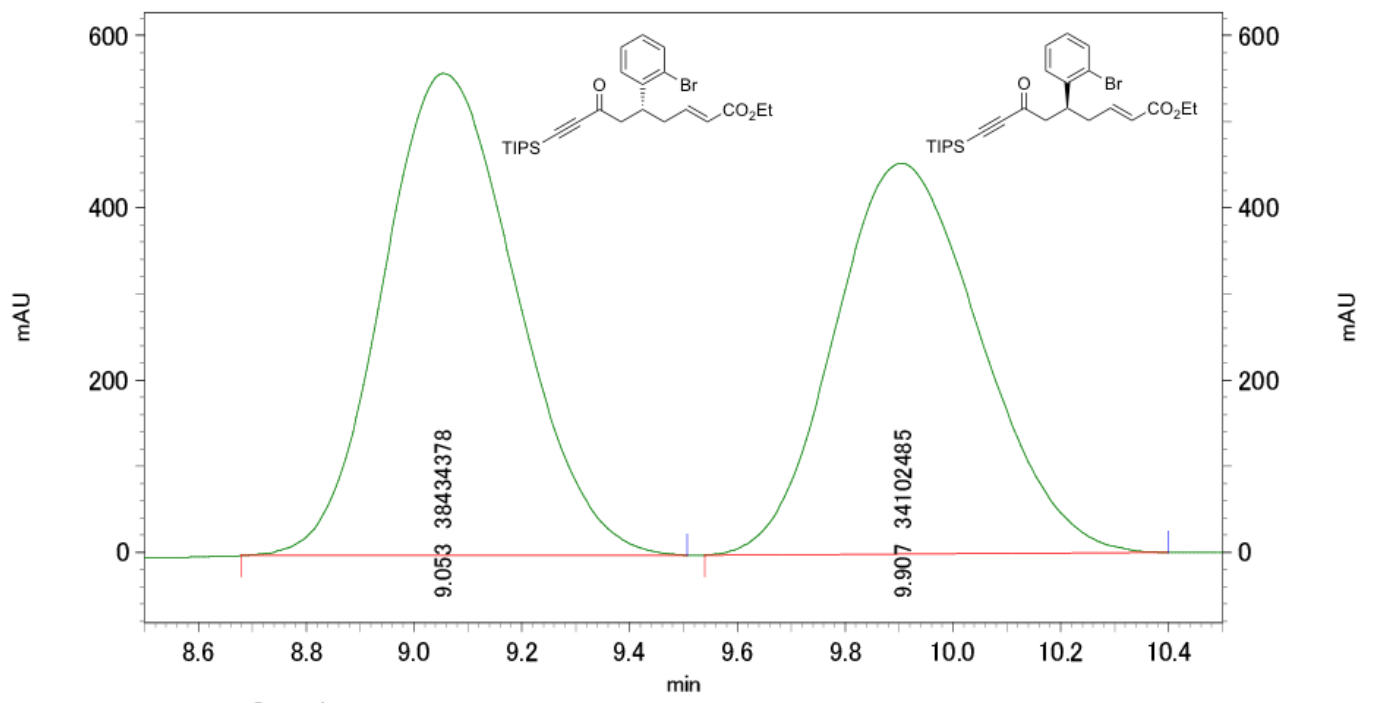

1: $234 \mathrm{~nm}, 4 \mathrm{~nm}$ 結果

Pk \# Retention time / min

Integration/\%

9.053

52.986
47.014

\begin{tabular}{|r|r|}
\hline$r-$ 㚈 & 100.000 \\
\hline
\end{tabular} 


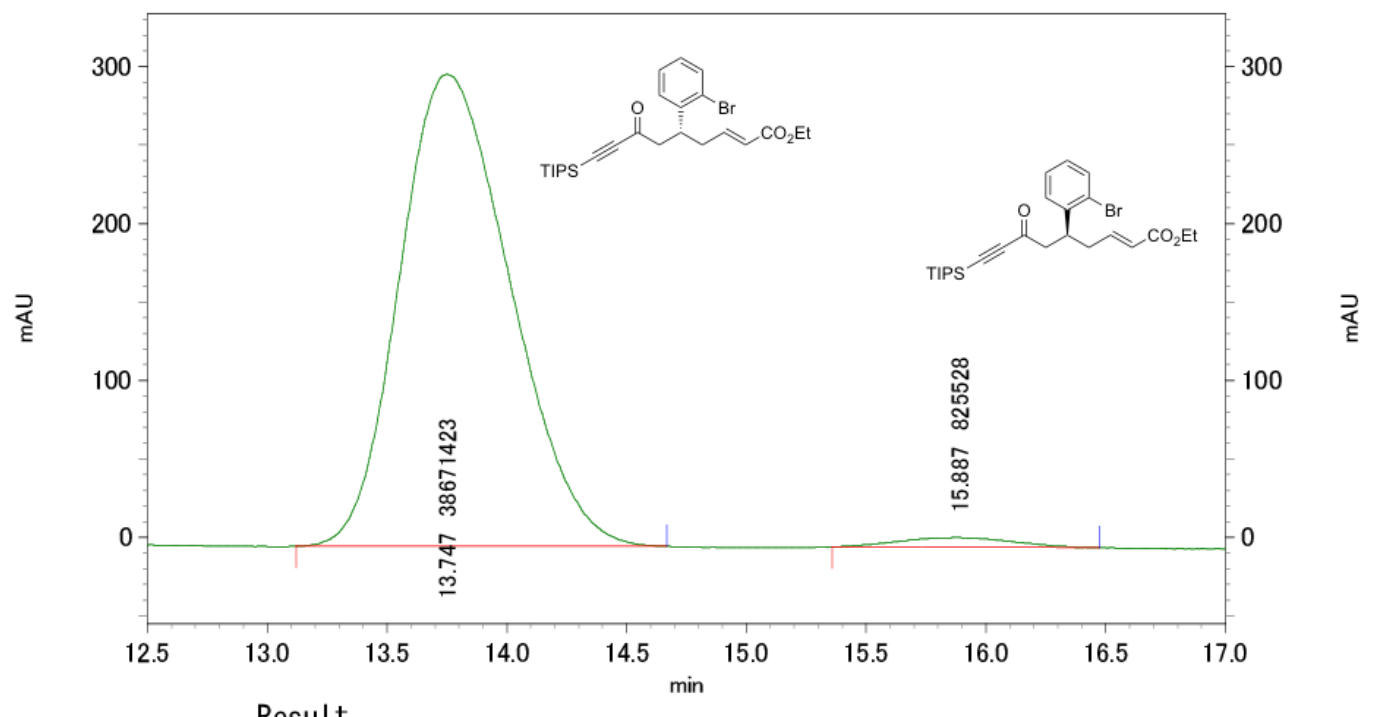

1: $234 \mathrm{~nm}, 4$ nm結果

Pk \# Retention time / min

13.747
15.887

Integration/\%

15. 887

2. 090

\begin{tabular}{|r|r|}
\hline$r-$ 舛 & 100.000 \\
\hline
\end{tabular} 


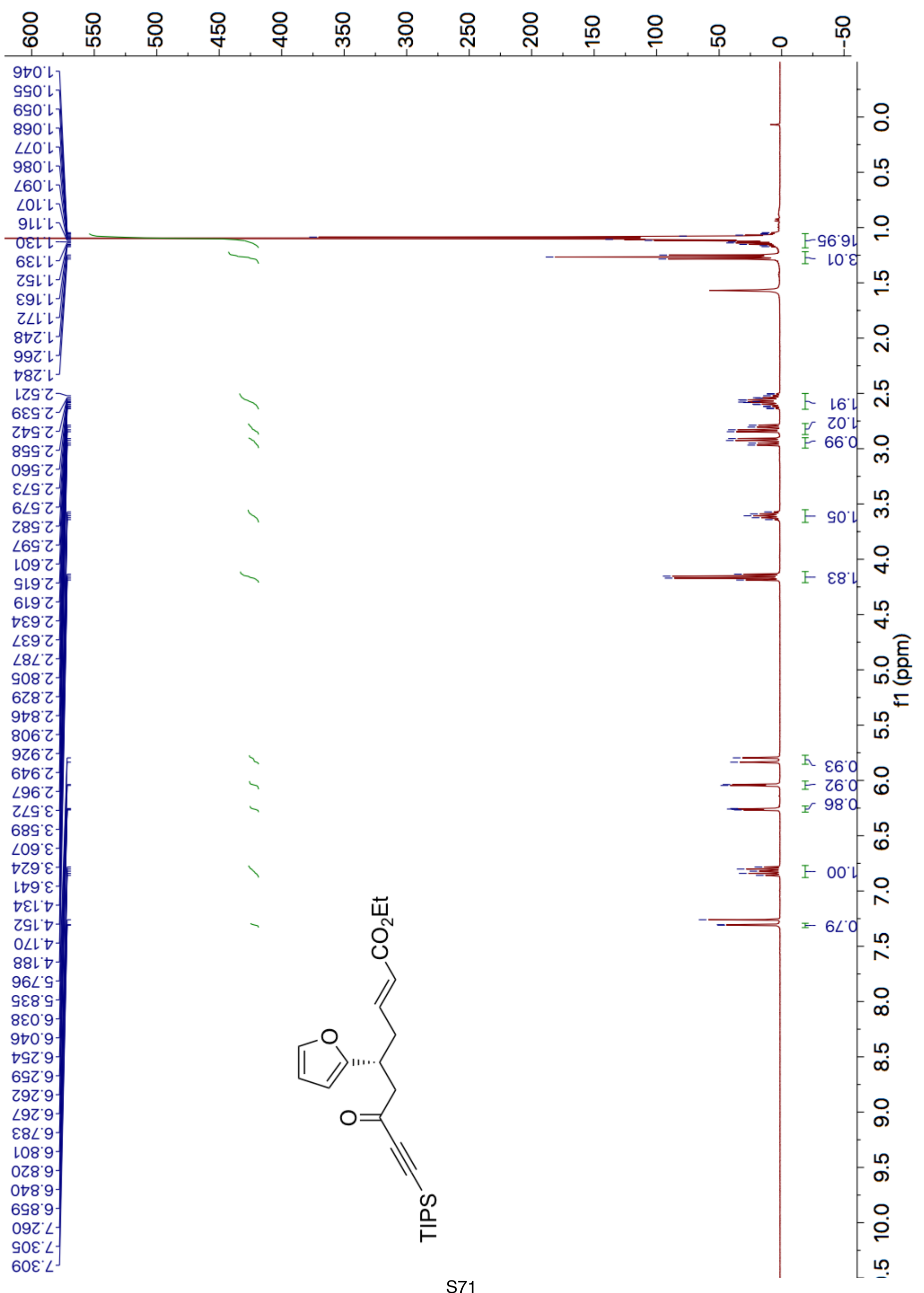




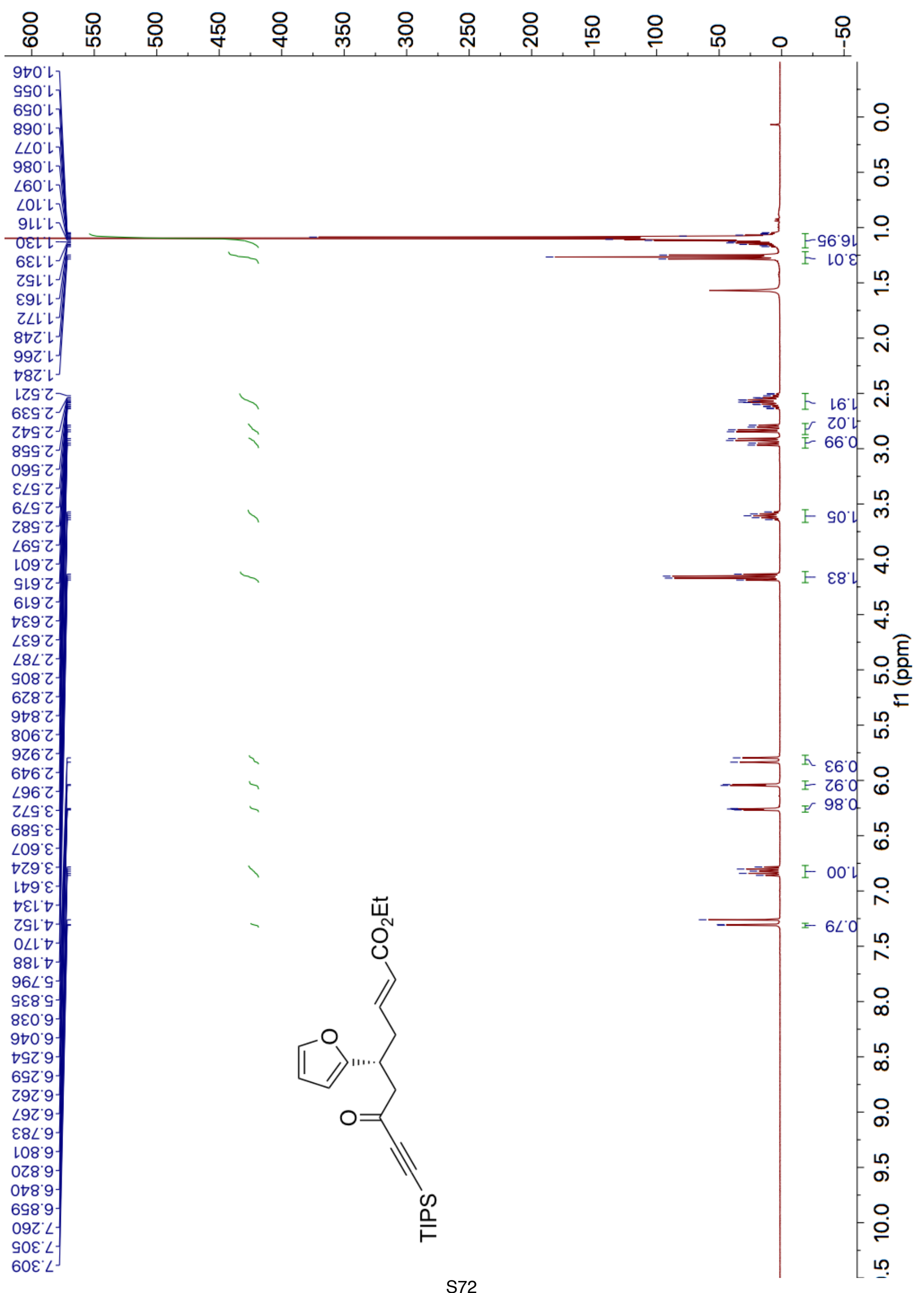




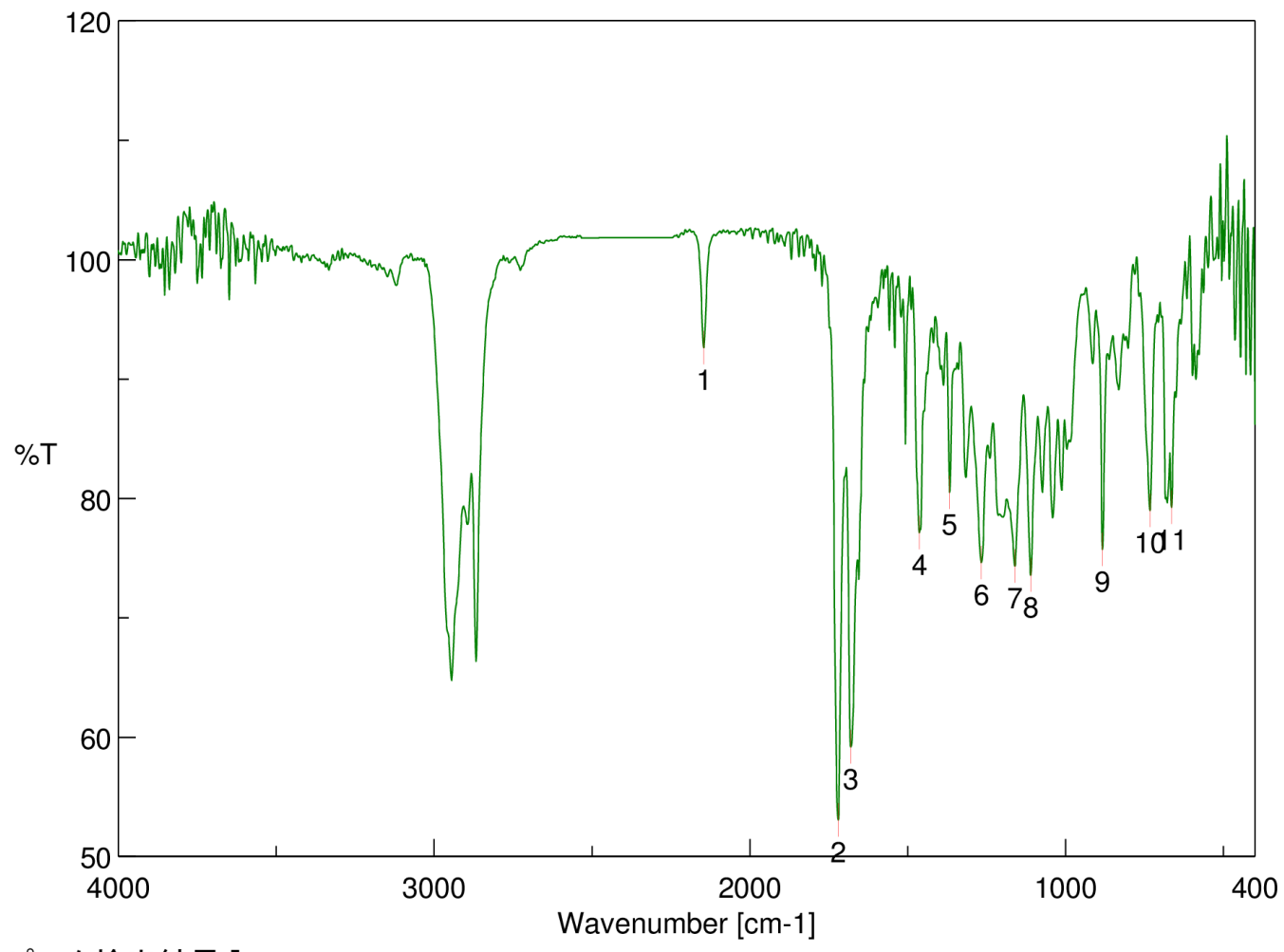

[ピーク検出結果 ]

$\begin{array}{llllll}\text { No. } & \text { 位置 } & \text { 強度 } & \text { No. } & \text { 位置 } & \text { 強度 } \\ 1 & 2146.38 & 92.6141 & 2 & 1720.19 & 53.0539 \\ 3 & 1680.66 & 59.1653 & 4 & 1463.71 & 77.1184 \\ 5 & 1367.28 & 80.4945 & 6 & 1267 & 74.6197 \\ 7 & 1160.94 & 74.3223 & 8 & 1109.83 & 73.5718 \\ 9 & 883.238 & 75.7183 & 10 & 732.817 & 78.9756\end{array}$

$\begin{array}{lll}11 & 664.357 \quad 79.2511\end{array}$<smiles>CCOC(=O)CCCc1ccco1</smiles> 


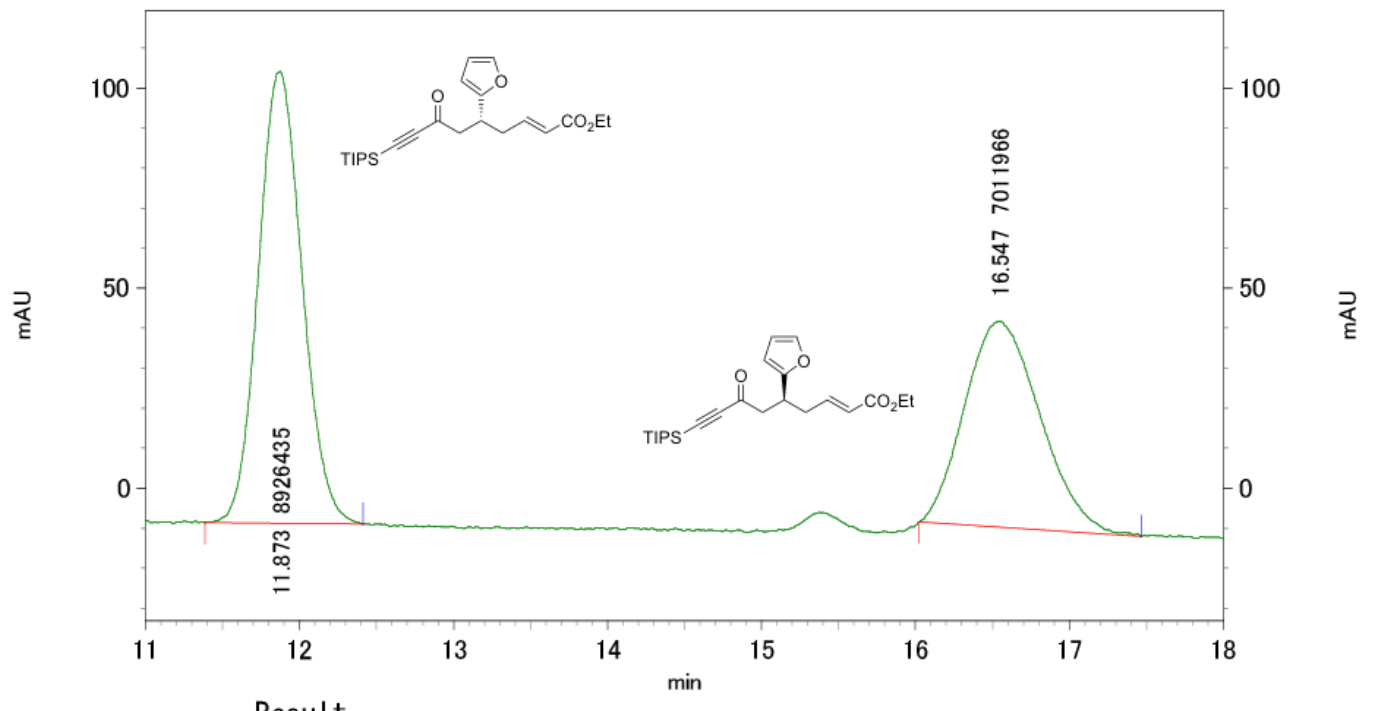

1: $213 \mathrm{~nm}, 4$ nm結果

Pk \# Retention time / min

11.873
16.547

Integration/ \%

\begin{tabular}{rrrr|}
\multicolumn{1}{r|}{ Pk \# } & Retention time $/$ min & Integration/\% \\
\hline 2 & 11.873 & 56.006 \\
\hline \multicolumn{2}{|c|}{} & 16.547 & 43.994 \\
\hline r-夕夕 & & 100.000 \\
\hline
\end{tabular}




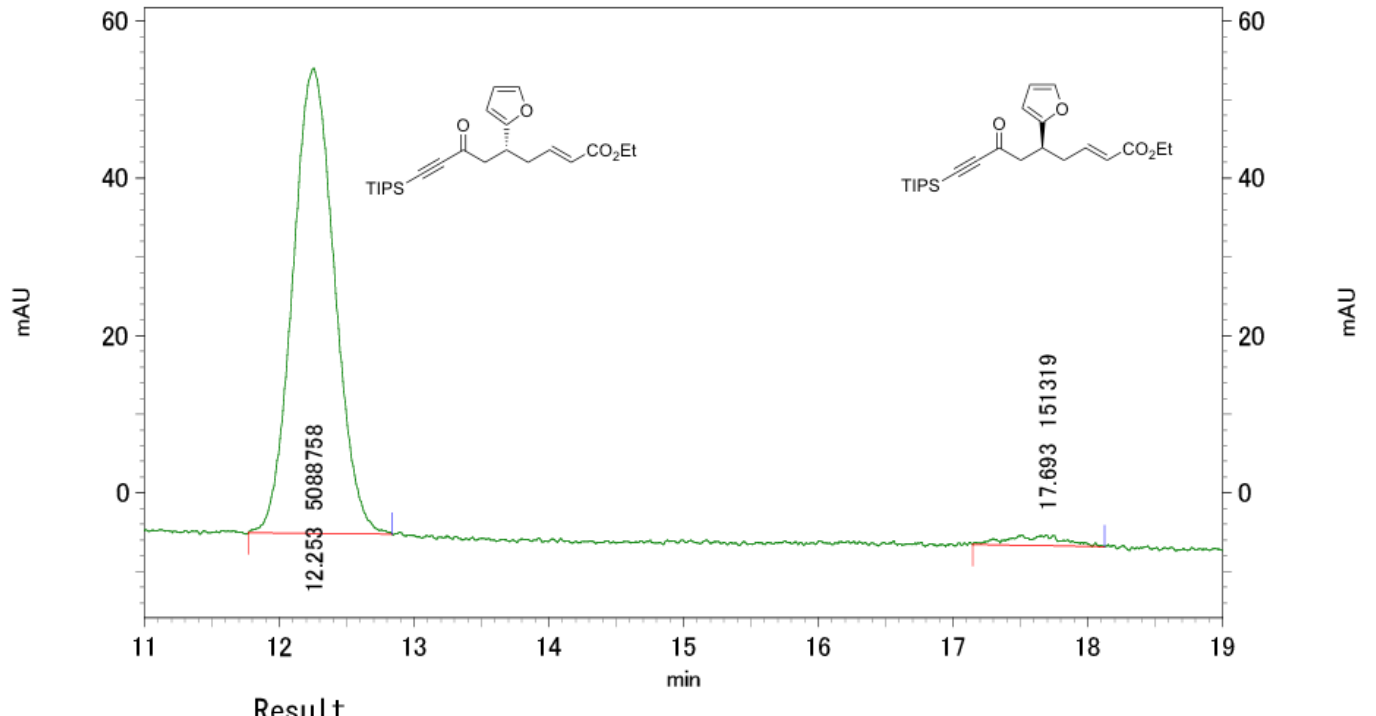

1: $213 \mathrm{~nm}, 4 \mathrm{~nm}$ 結果

\begin{tabular}{|r|r|}
\hline$r-$ 㚈 & 100.000 \\
\hline
\end{tabular}




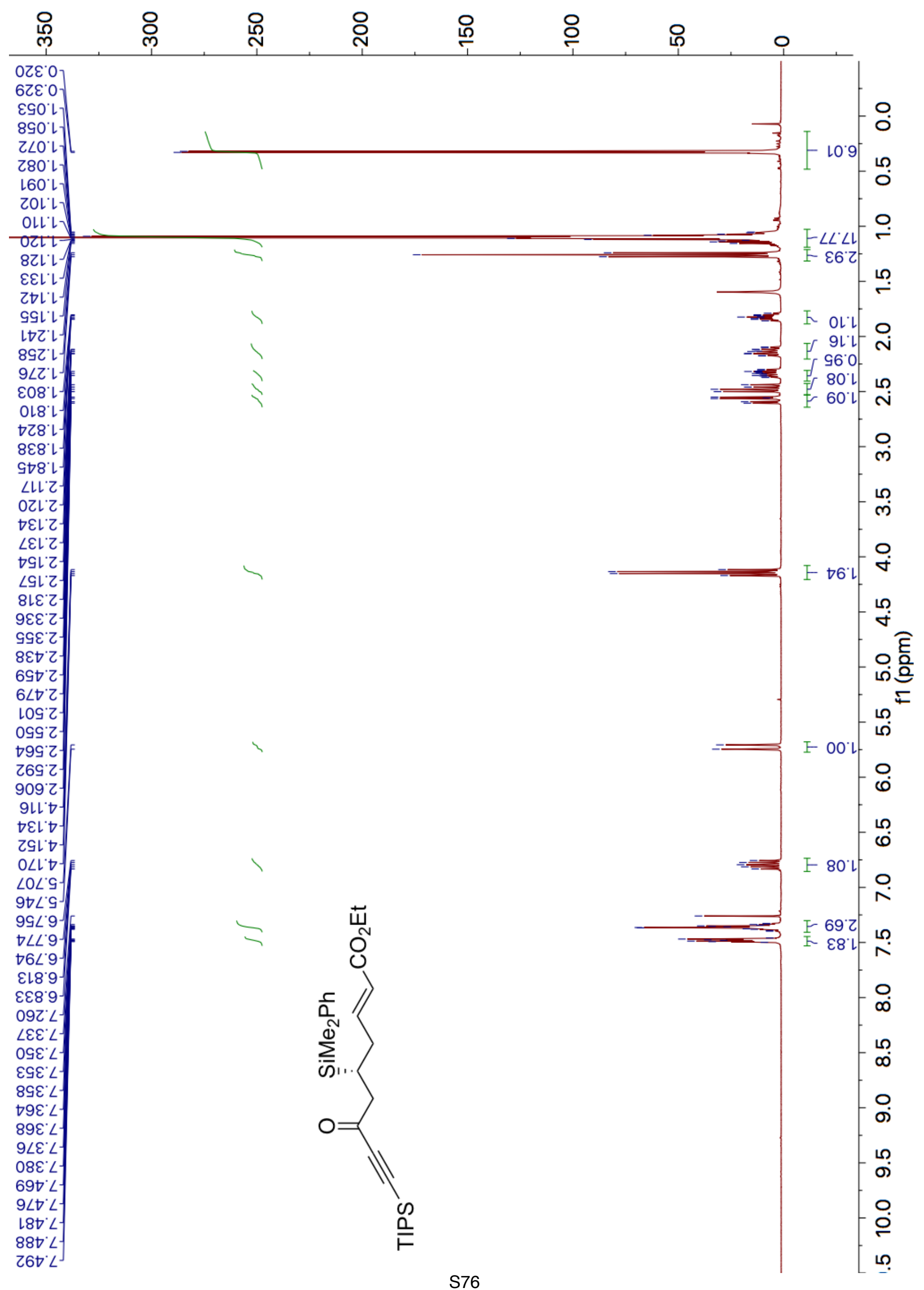




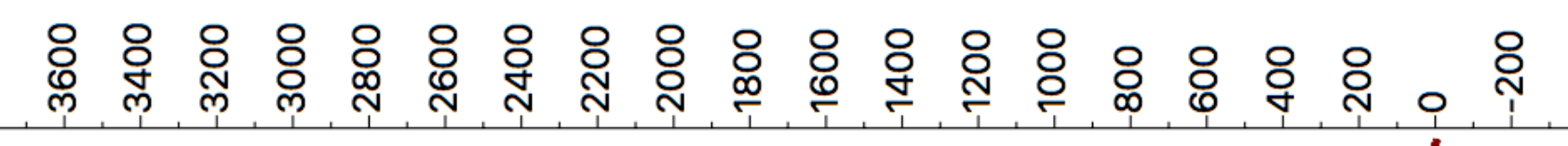

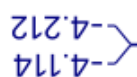

$\nabla \angle 6^{\circ} \mathrm{OL}-$

60でレー

$\angle \angle D^{\circ} 8 \mathrm{~L}=$

ISL'LC-

6เ9'Zะ-

เ89'9t-

Zll09-

189.94

$000 \angle L T$

$8 \mathrm{~L} \varepsilon^{\circ} L L$

Oll'96-

960 เOL-

$\angle Z L Z Z L-$

$896 \mathrm{LZL}$

$6 \angle \varepsilon^{\circ} 6 Z L-$

098 $\varepsilon \varepsilon\llcorner-$

OZL'9عL-

$99 \angle \angle \nabla L-$

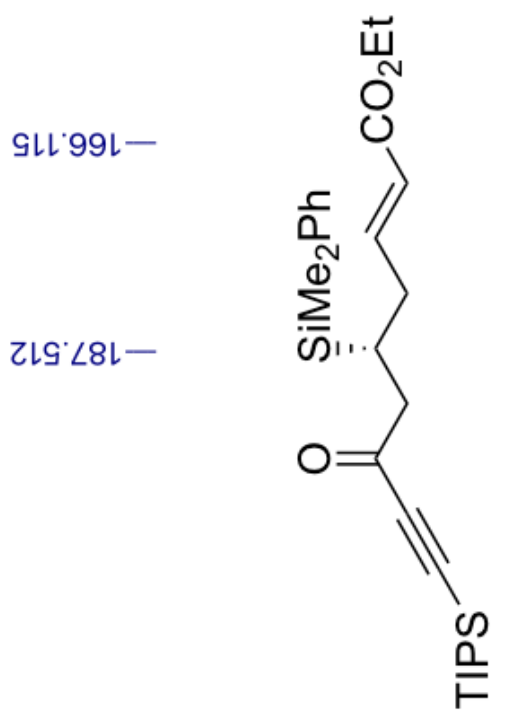




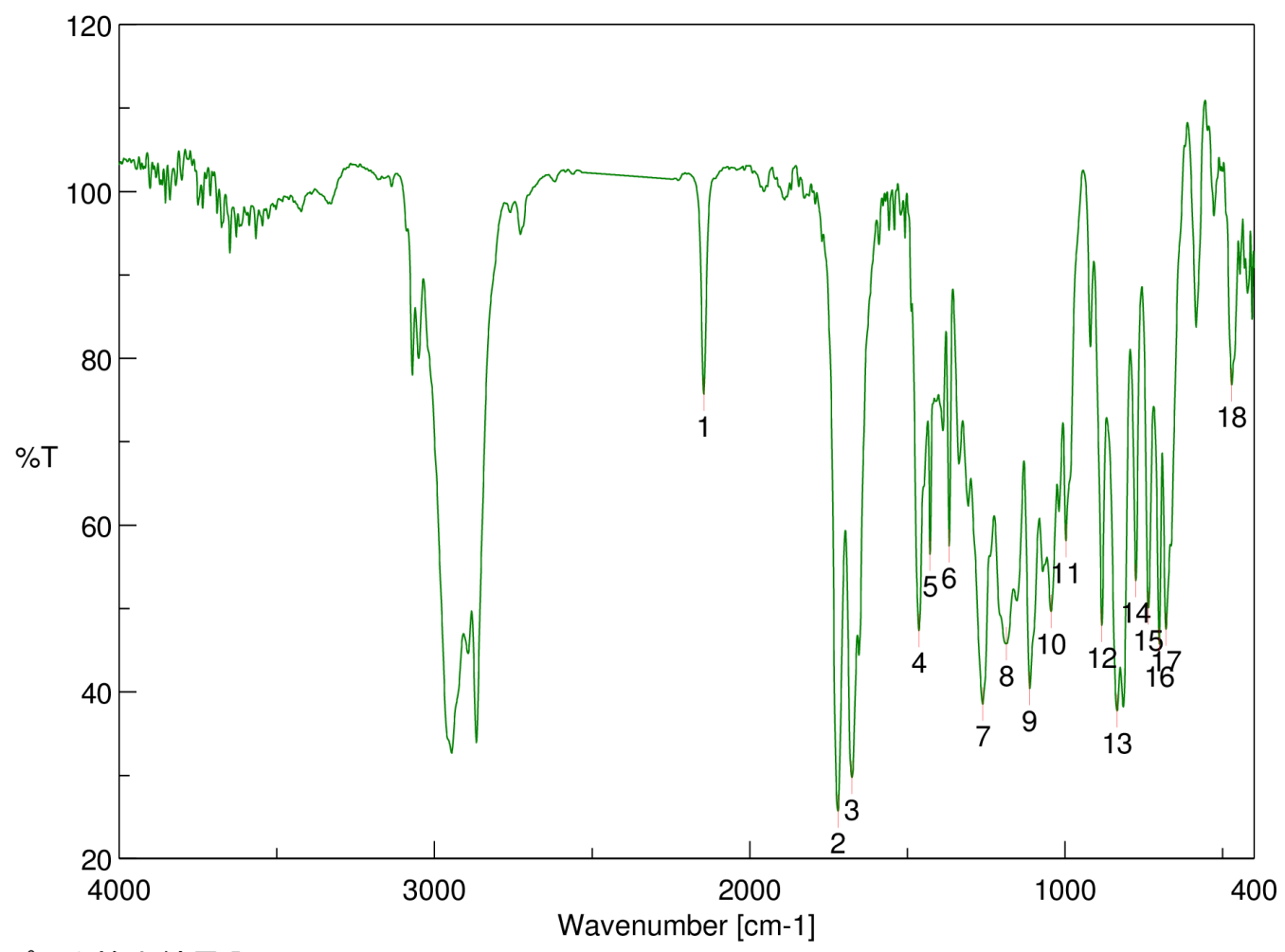

\section{[ピーク検出結果 ]}

$\begin{array}{llllll}\text { No. } & \text { 位置 } & \text { 強度 } & \text { No. } & \text { 位置 } & \text { 強度 } \\ 1 & 2145.42 & 75.6876 & 2 & 1720.19 & 25.7176 \\ 3 & 1675.84 & 29.7196 & 4 & 1463.71 & 47.3614 \\ 5 & 1428.03 & 56.479 & 6 & 1367.28 & 57.4964 \\ 7 & 1261.22 & 38.5372 & 8 & 1186.01 & 45.7387 \\ 9 & 1111.76 & 40.3718 & 10 & 1044.26 & 49.6385 \\ 11 & 997.017 & 58.1297 & 12 & 883.238 & 47.9641 \\ 13 & 835.026 & 37.7479 & 14 & 775.244 & 53.3237 \\ 15 & 735.71 & 50.0408 & 16 & 700.998 & 45.7245 \\ 17 & 679.785 & 47.497 & 18 & 471.51 & 76.8163\end{array}$<smiles></smiles> 


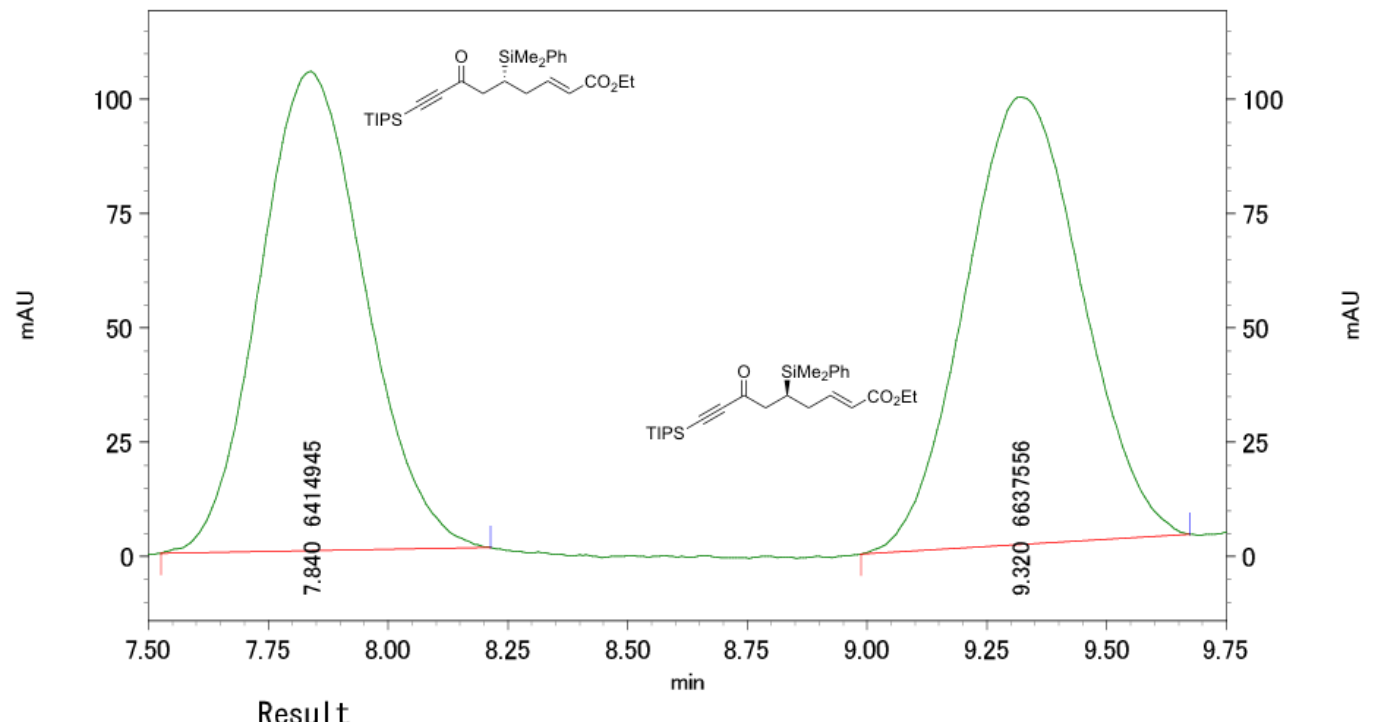

1: $213 \mathrm{~nm}, 4 \mathrm{~nm}$ 結果

\begin{tabular}{|r|r|}
\hline$r-$ 㚈 & 100.000 \\
\hline
\end{tabular}




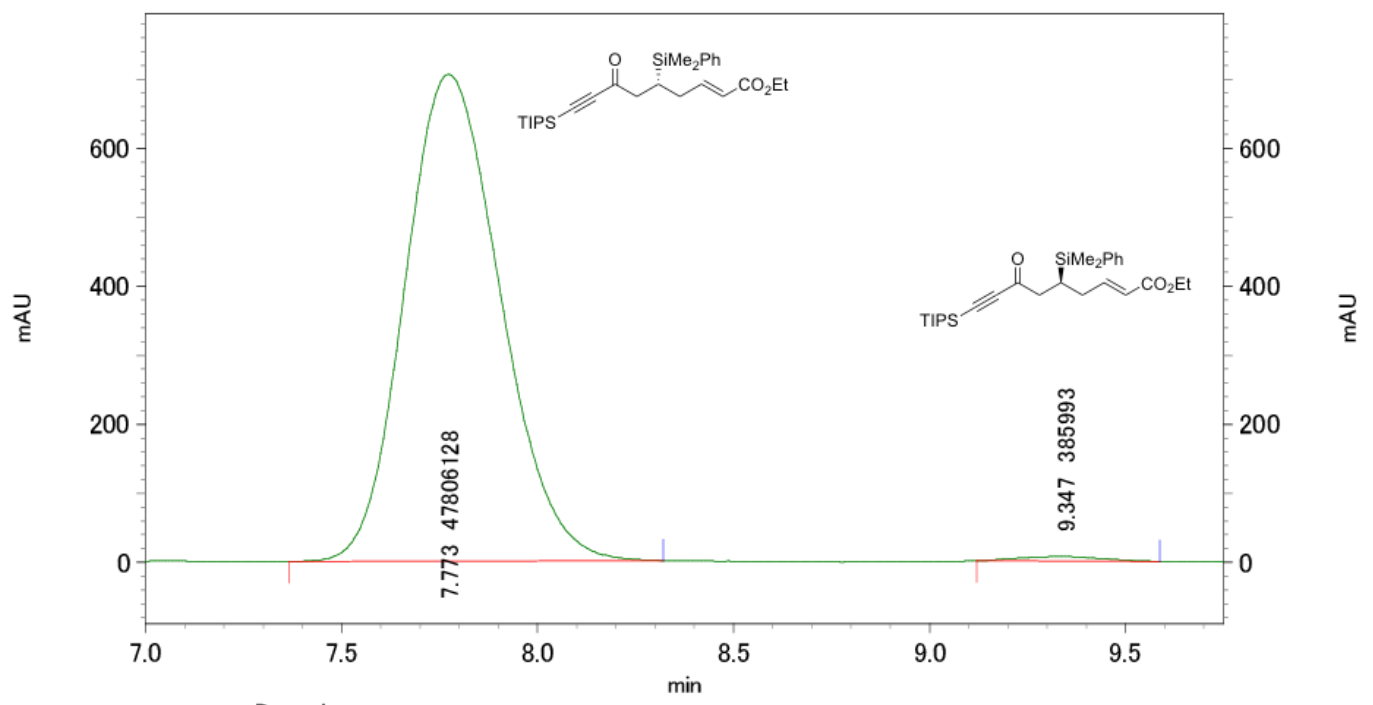

1: $213 \mathrm{~nm}, 4 \mathrm{~nm}$ 結果

Pk \# Retention time / min

9.347

Integration/ \%

0.801

\begin{tabular}{|r|r|}
\hline$r-$ 㚈 & 100.000 \\
\hline
\end{tabular} 


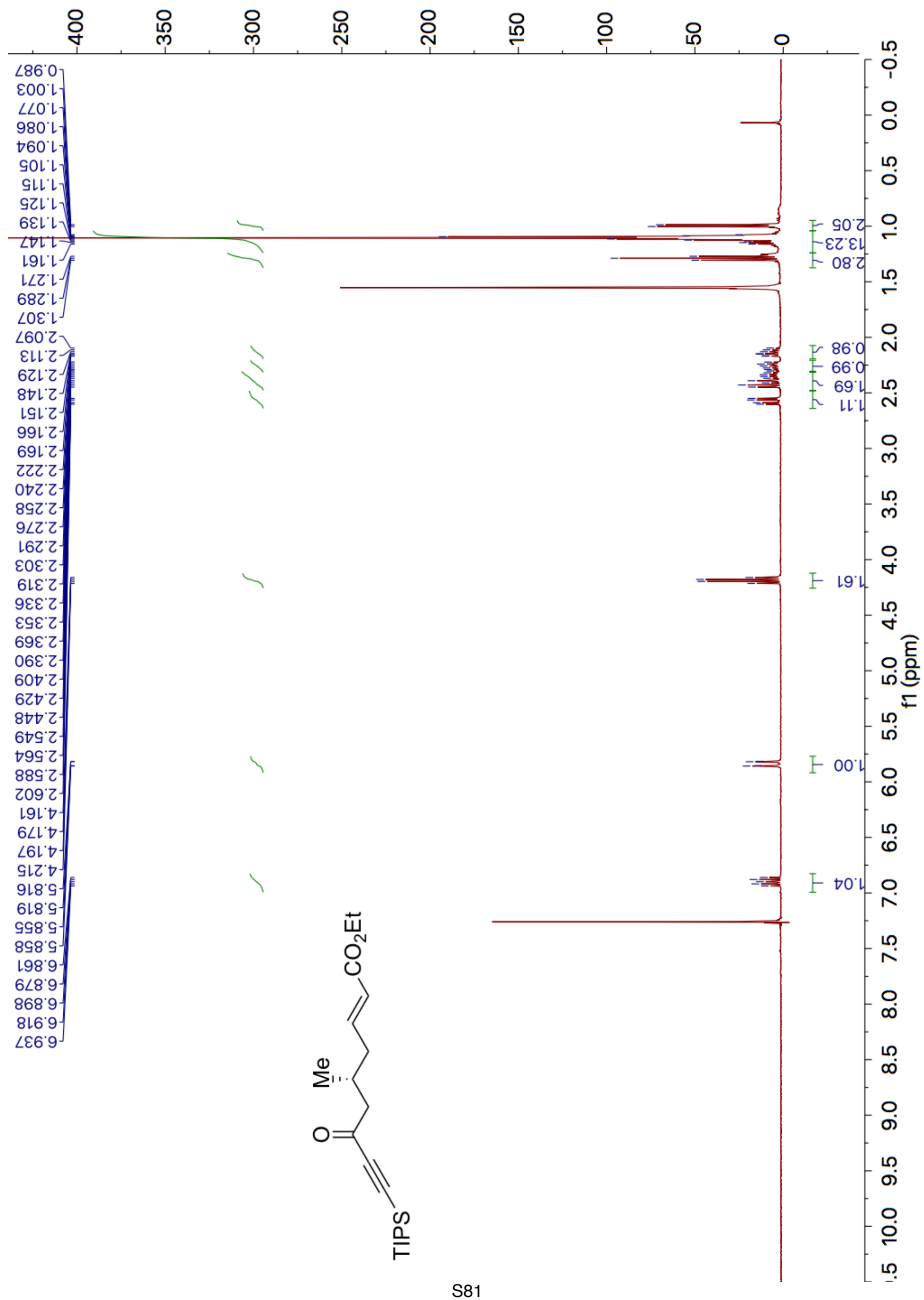


各 兽

Ot6.0L

てヤでヤレ二

†978

$109^{\circ} 6 \mathrm{~L}^{-}$

งเย*6乙-

$\varepsilon \succsim 6 \cdot 8 \varepsilon-$

$\triangleright 90^{\circ} 29-$

$189.9 /$

$000 \angle L \frac{1}{5}$

$8 L \varepsilon^{\prime} L L$

$7966^{\circ} 96-$

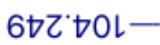

$\nabla \angle \varepsilon^{\cdot} \varepsilon 乙 L-$

\&9ع'9ヤا—

$9 \nabla \varepsilon \cdot 99 l-$

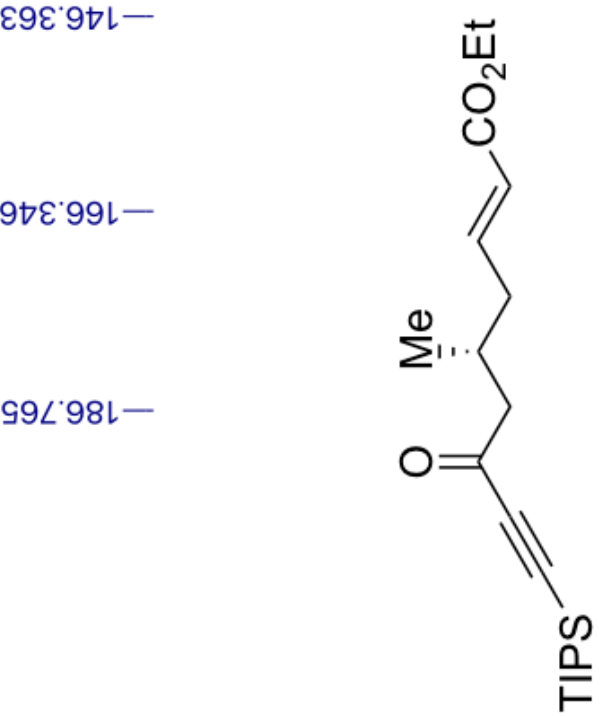




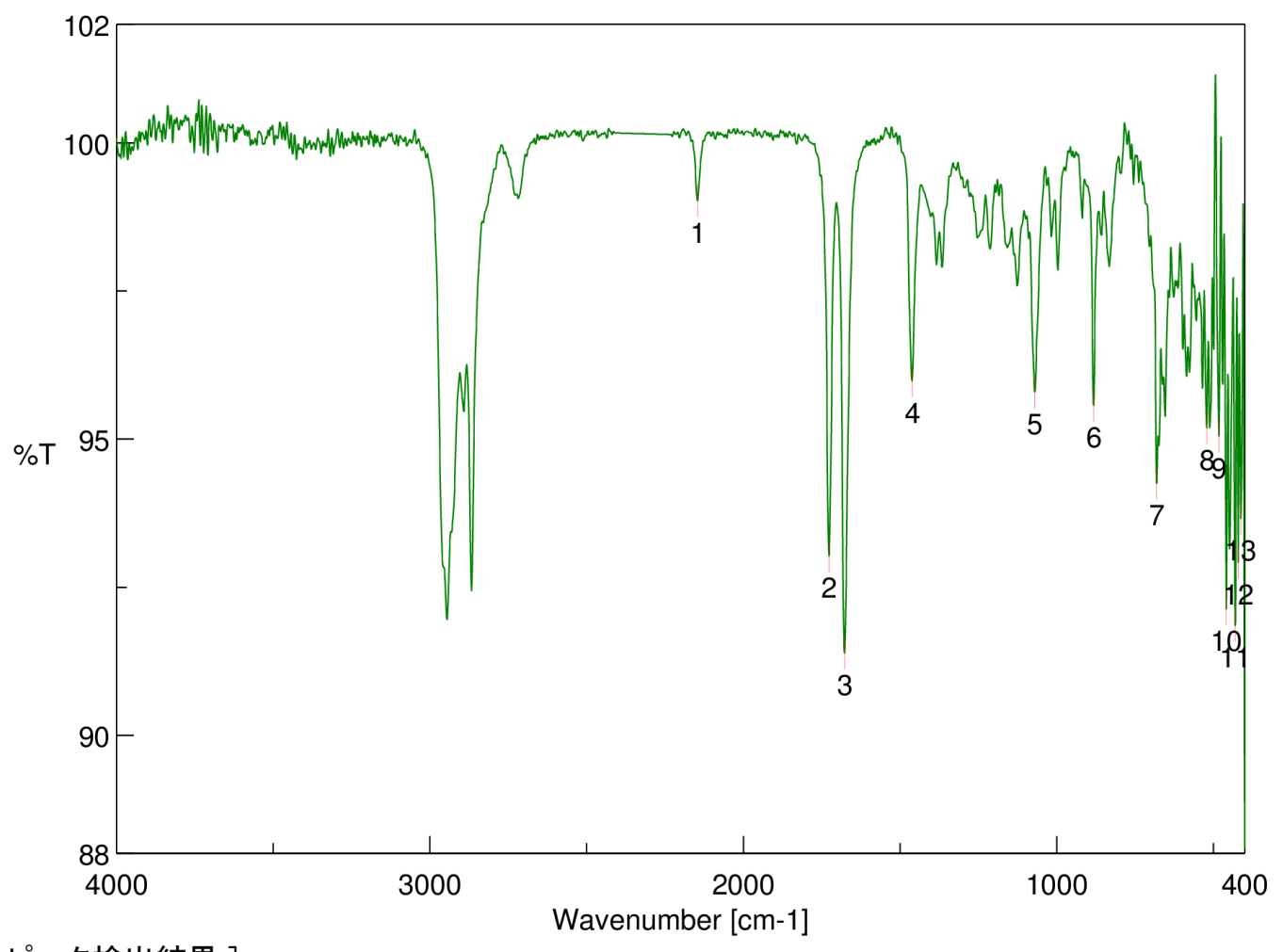

[ピーク検出結果 ]

$\begin{array}{llllll}\text { No. } & \text { 位置 } & \text { 強度 } & \text { No. } & \text { 位置 } & \text { 強度 } \\ 1 & 2146.38 & 99.0249 & 2 & 1726.94 & 93.0258 \\ 3 & 1676.8 & 91.3858 & 4 & 1461.78 & 95.983 \\ 5 & 1070.3 & 95.798 & 6 & 882.274 & 95.5699 \\ 7 & 681.713 & 94.2521 & 8 & 521.65 & 95.1872 \\ 9 & 483.081 & 95.0478 & 10 & 458.975 & 92.1311 \\ 11 & 431.012 & 91.8513 & 12 & 421.37 & 92.919 \\ 13 & 412.692 & 93.659 & & & \end{array}$

$13 \quad 412.692 \quad 93.659$

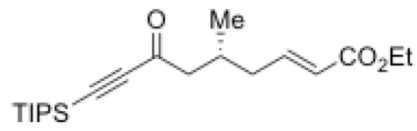




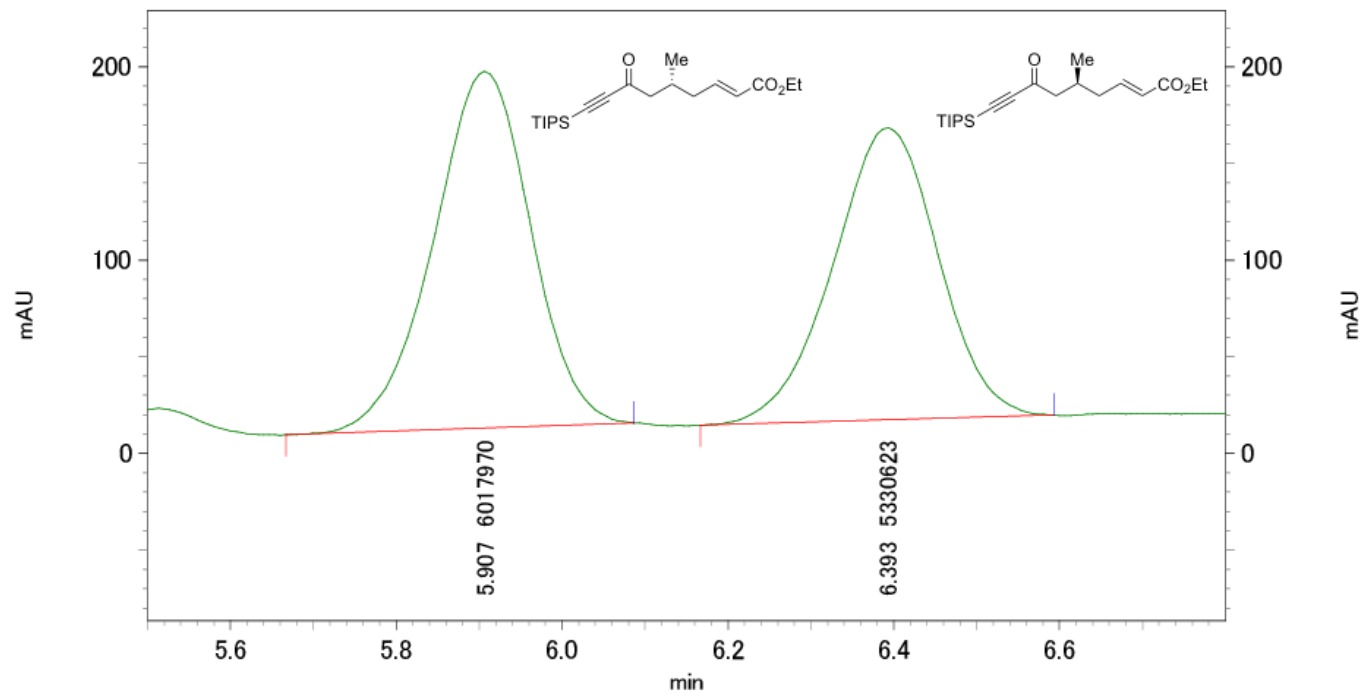

Result

1: $213 \mathrm{~nm}, 4 \mathrm{~nm}$ 結果

Integration/\%

6. 393

46.972

\begin{tabular}{|r|r|}
\hline$r-$ 外 & 100.000 \\
\hline Total
\end{tabular} 


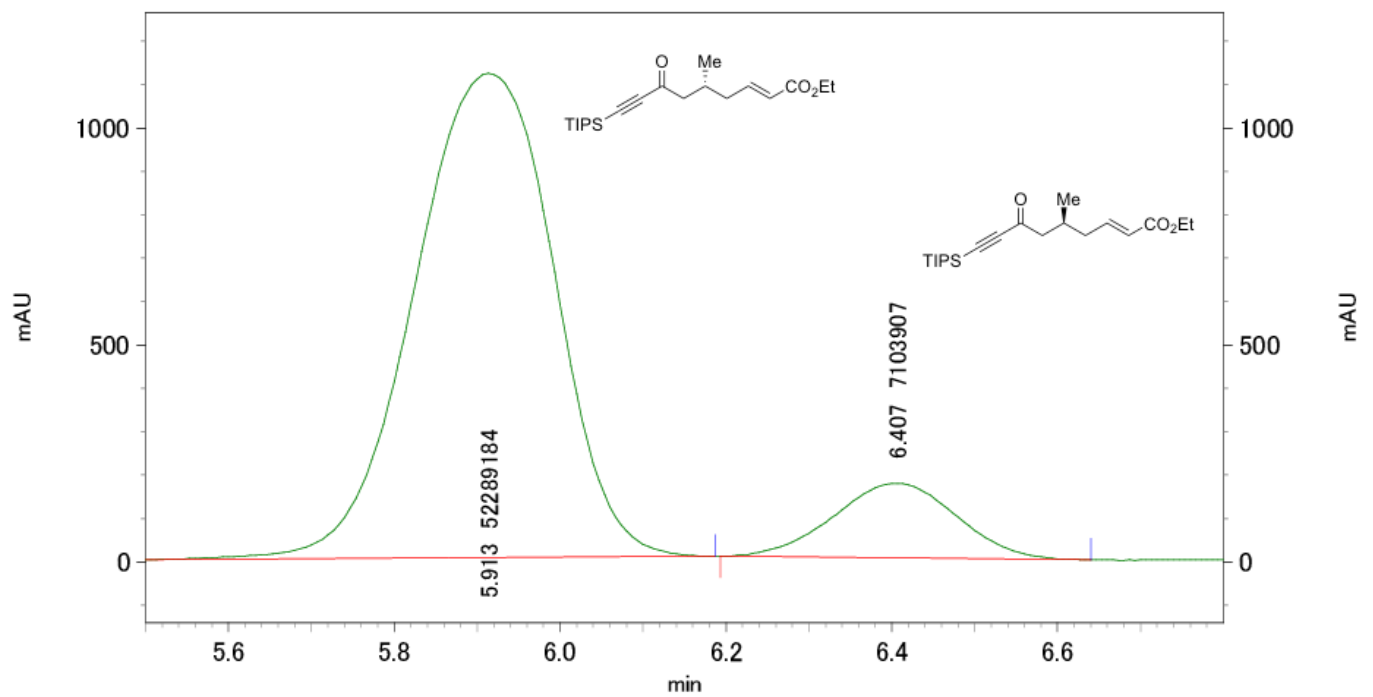

Result

1: $213 \mathrm{~nm}, 4 \mathrm{~nm}$ 結果

Integration/\%

6. 407

11.961

\begin{tabular}{|r|r|r|}
\hline$r-$ 多 & 100.000 \\
\hline
\end{tabular} 


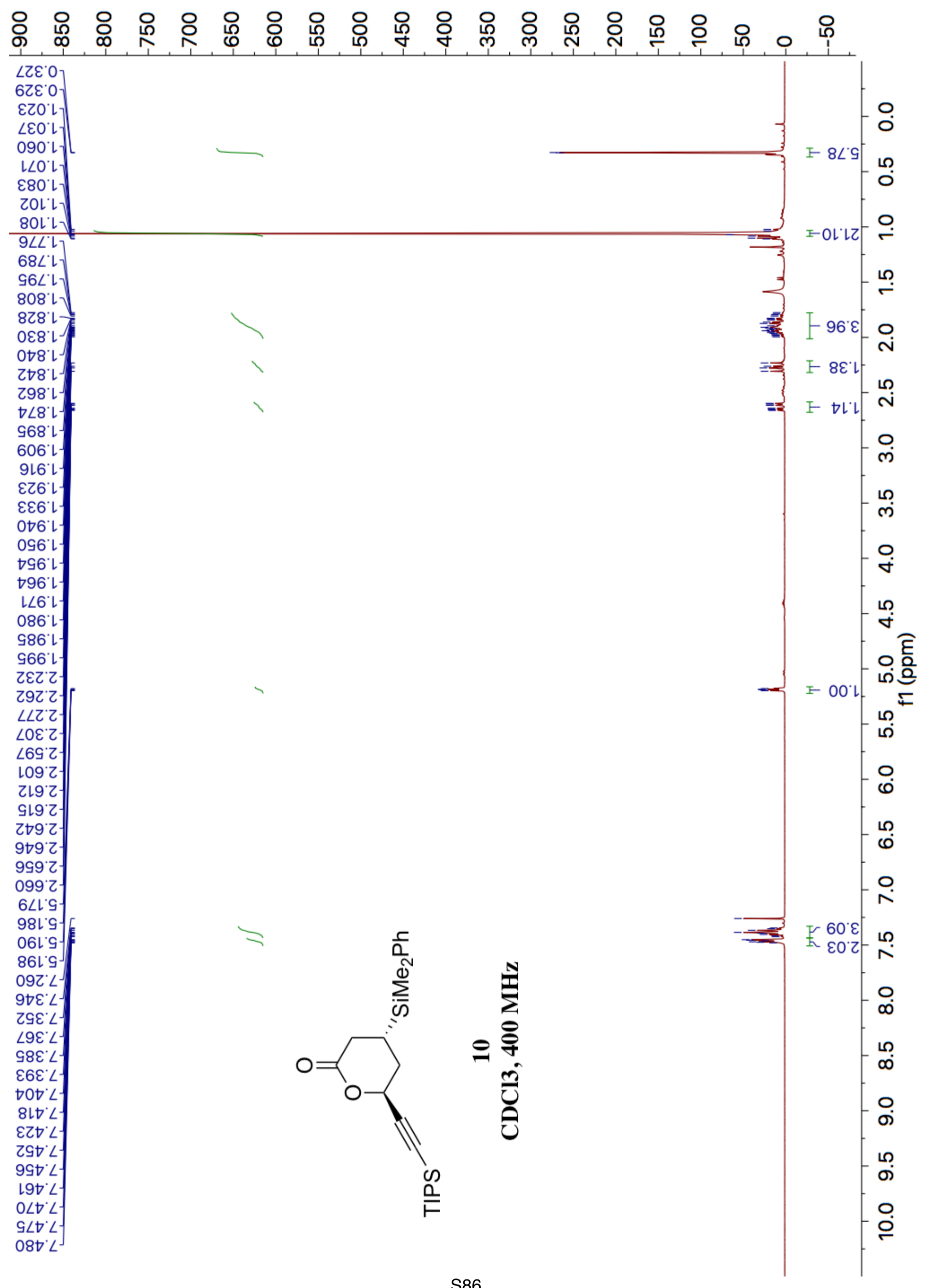




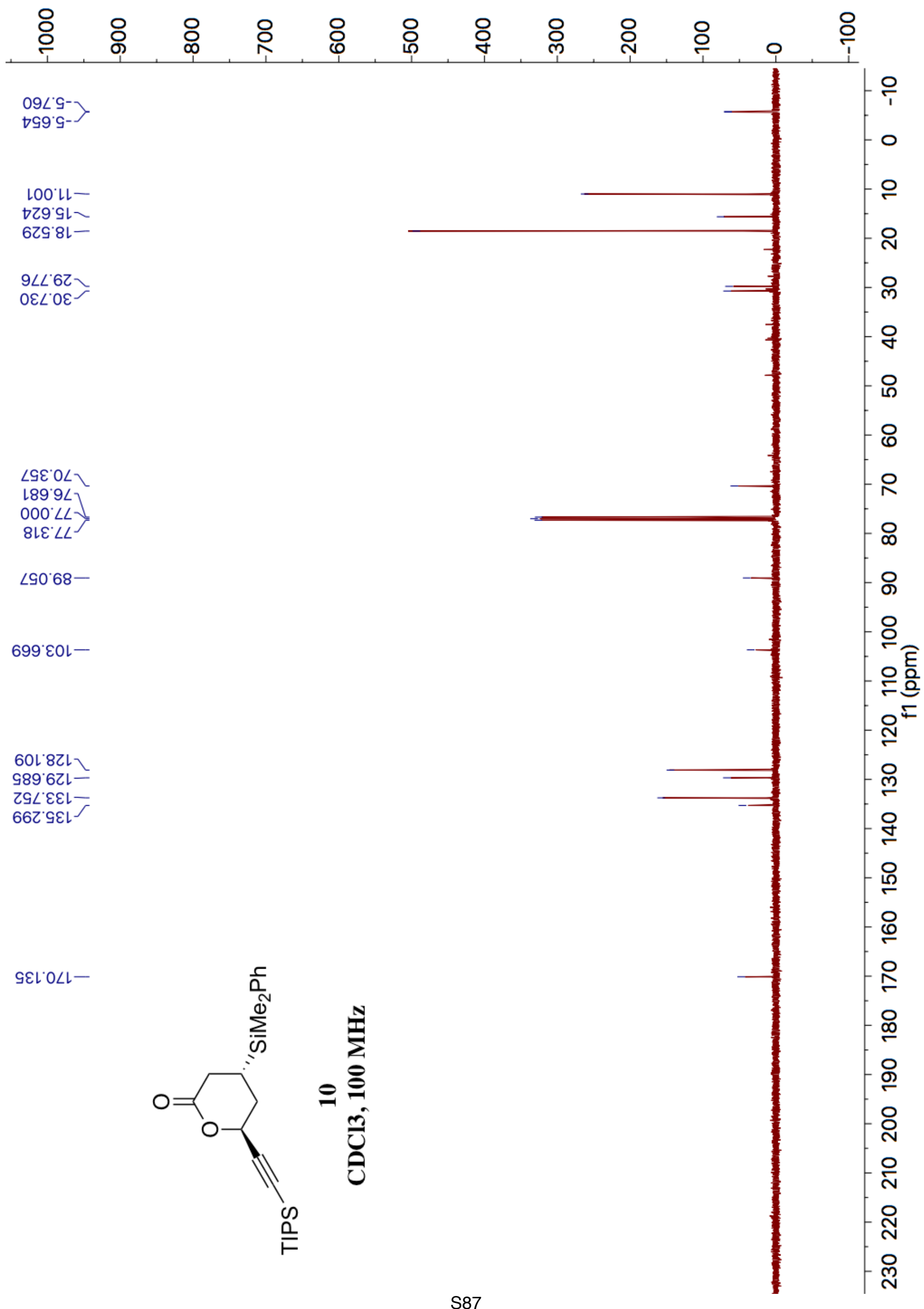




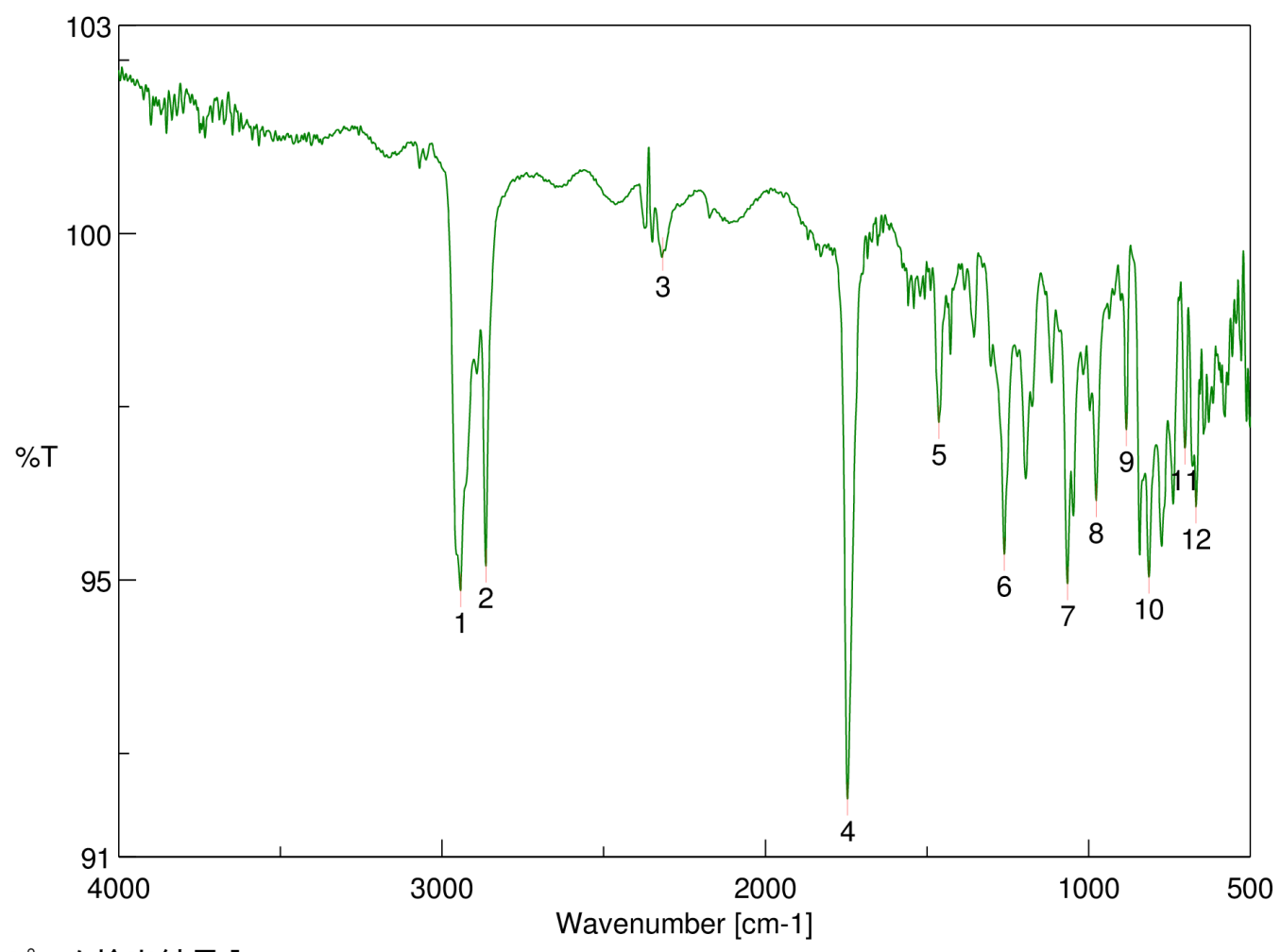

[ピーク検出結果 ]

$\begin{array}{llllll}\text { No. } & \text { 位置 } & \text { 強度 } & \text { No. } & \text { 位置 } & \text { 強度 } \\ 1 & 2942.84 & 94.8446 & 2 & 2864.74 & 95.2006 \\ 3 & 2317.05 & 99.6995 & 4 & 1746.23 & 91.8394 \\ 5 & 1463.71 & 97.2725 & 6 & 1261.22 & 95.3701 \\ 7 & 1065.48 & 94.9429 & 8 & 976.769 & 96.146 \\ 9 & 883.238 & 97.1658 & 10 & 813.813 & 95.0414 \\ 11 & 701.962 & 96.9027 & 12 & 668.214 & 96.0529\end{array}$

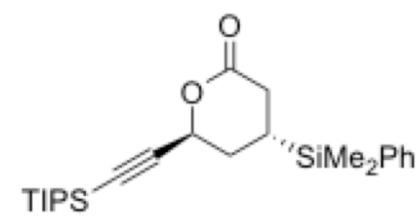




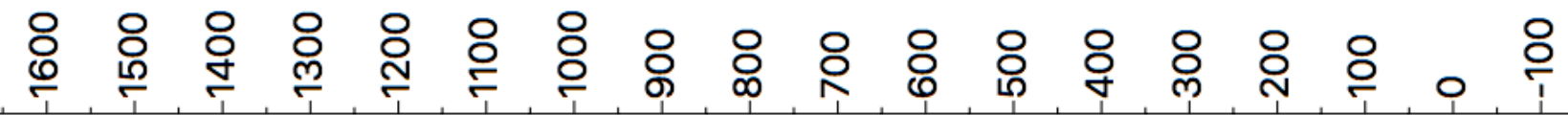

ZZ๐'

$\varepsilon \angle \nabla^{\circ}\llcorner$

$\angle 99^{\circ} \mathrm{L}$

969'

$\supset Z 8^{\prime} L$

0E8'

$\angle E 8^{\prime} \mathrm{L}$

998'

ع98' $\rightarrow$

698.

$\angle L \varepsilon^{\prime} 乙$

Z6\&'乙

レレてて

乙E† て

LStて

乙9॰乙

$\angle E G^{\prime} 乙$

$\mathrm{GS}$ '

$9 \angle 9^{\prime} Z$

$\nabla 69^{\circ}{ }^{\top}$

$889^{\circ} \varepsilon$

$8 \angle{ }^{\prime} \downarrow$

G8Z't

G6乙 $\nabla$

$00 \varepsilon+\nabla$

$80 \varepsilon$ '

แย่

$\varepsilon เ \varepsilon^{\prime} \triangleright$

$\angle L \varepsilon^{\prime} \nabla$

๑と'॰

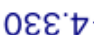

O๑६'ฑ

$9 \nabla \varepsilon \triangleright \nabla$

$\angle 99^{\circ} \mathrm{D}$

$\varepsilon \angle 9^{\circ} \nabla$

$6 \angle 9^{\circ} \nabla$

969" $\nabla$

ZOL't

$80 \angle ' t$

$092 \angle$
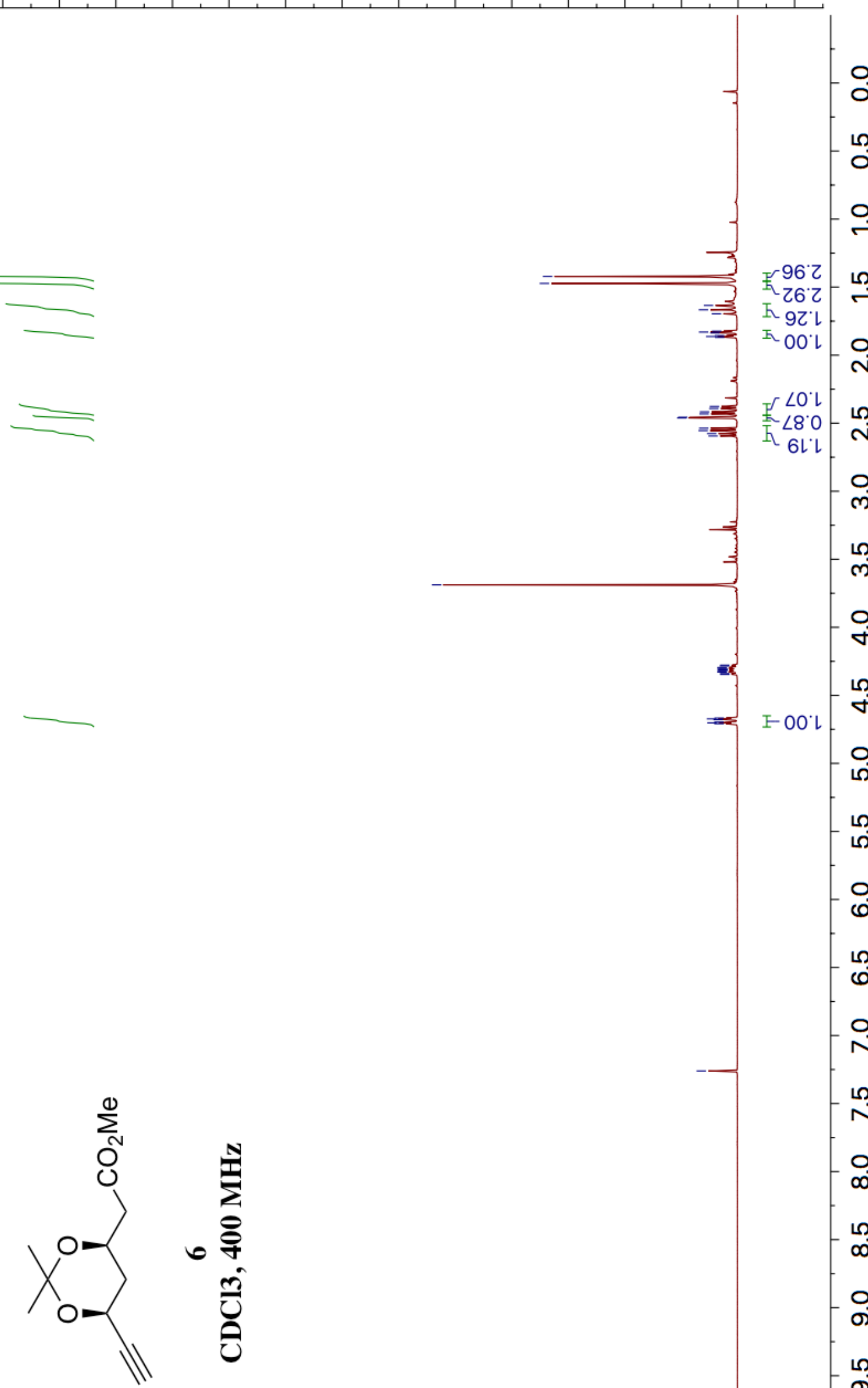


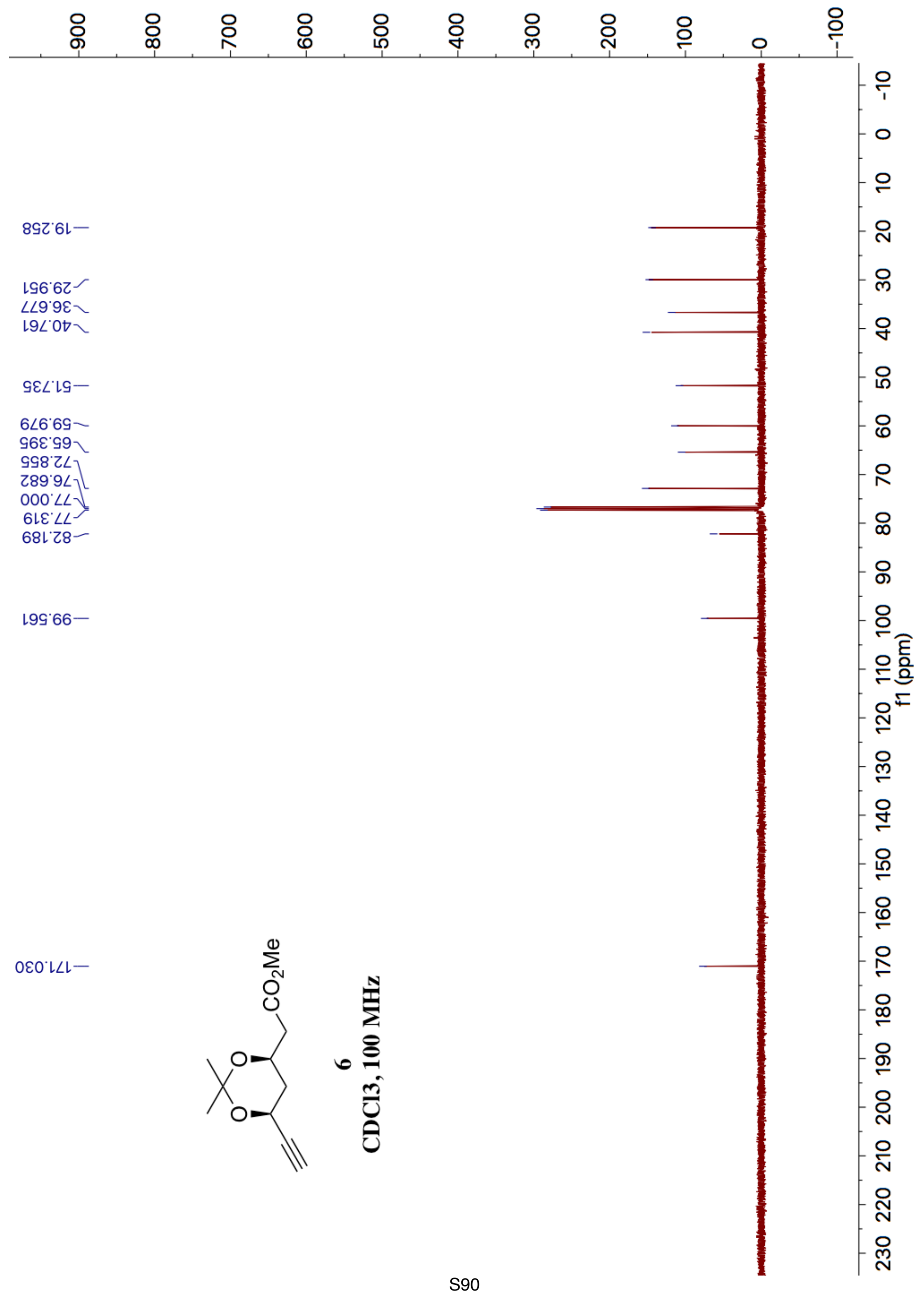




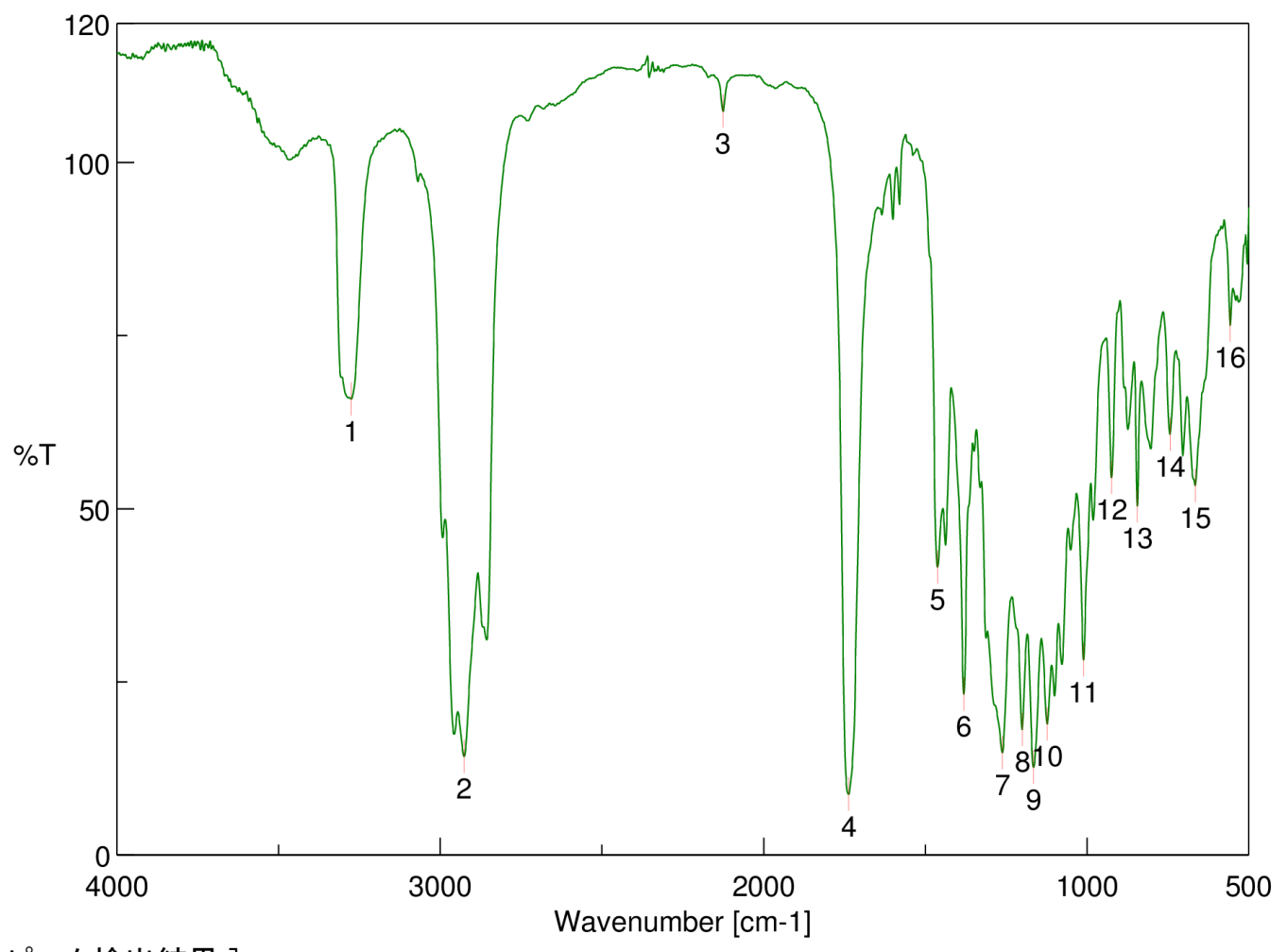

[ピーク検出結果 ]

$\begin{array}{llllll}\text { No. } & \text { 位置 } & \text { 強度 } & \text { No. } & \text { 位置 } & \text { 強度 } \\ 1 & 3275.5 & 65.7972 & 2 & 2926.45 & 14.2363 \\ 3 & 2126.13 & 107.341 & 4 & 1737.55 & 8.77249 \\ 5 & 1462.74 & 41.5256 & 6 & 1380.78 & 23.2454 \\ 7 & 1262.18 & 14.8 & 8 & 1200.47 & 18.1388 \\ 9 & 1165.76 & 12.663 & 10 & 1123.33 & 18.9334 \\ 11 & 1010.52 & 28.1406 & 12 & 923.736 & 54.4708 \\ 13 & 844.669 & 50.3803 & 14 & 743.424 & 60.7057 \\ 15 & 665.321 & 53.3182 & 16 & 557.327 & 76.4504\end{array}$<smiles>C#CC1CC(CC(C)=O)OC(C)(C)O1</smiles> 


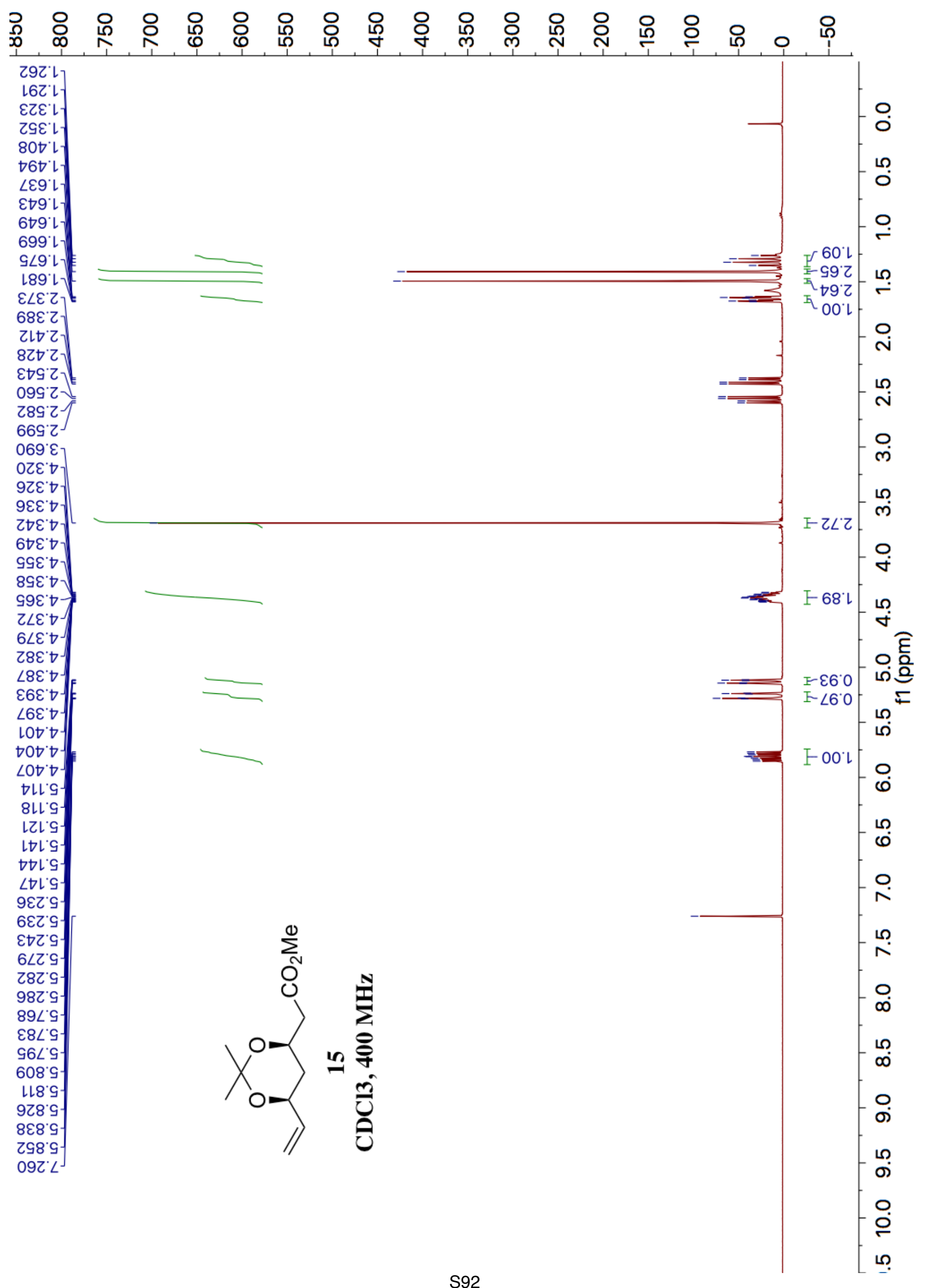




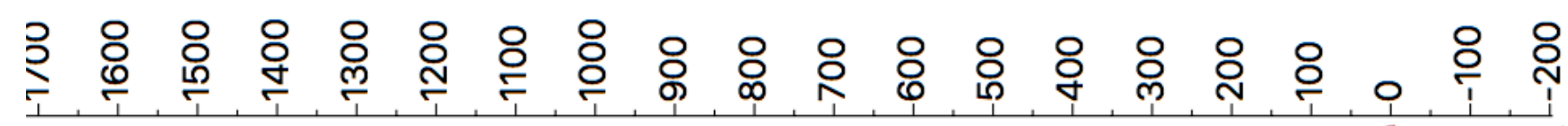

969.6L-

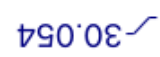

$8 \nabla z \cdot 9 \varepsilon-$

Stlito

899'L-

¿E9 99 ᄀ

† $6^{\circ} 69$

$189^{\circ} 9 /$

$000 \angle L$

$81 \varepsilon^{\circ} \angle L$

$\angle \varepsilon^{\circ}{ }^{\circ}{ }^{-}-$

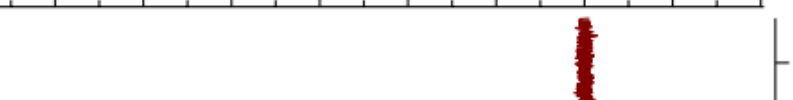

우

○

으

요

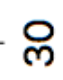

ㅇ

은

8

웅

$\infty$

요

으

zz9'งเL-

$\varepsilon 8 \varepsilon^{\prime} 8 \varepsilon\llcorner-$

StE $\downarrow L L-$

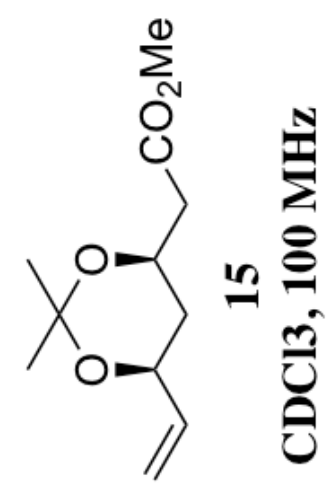




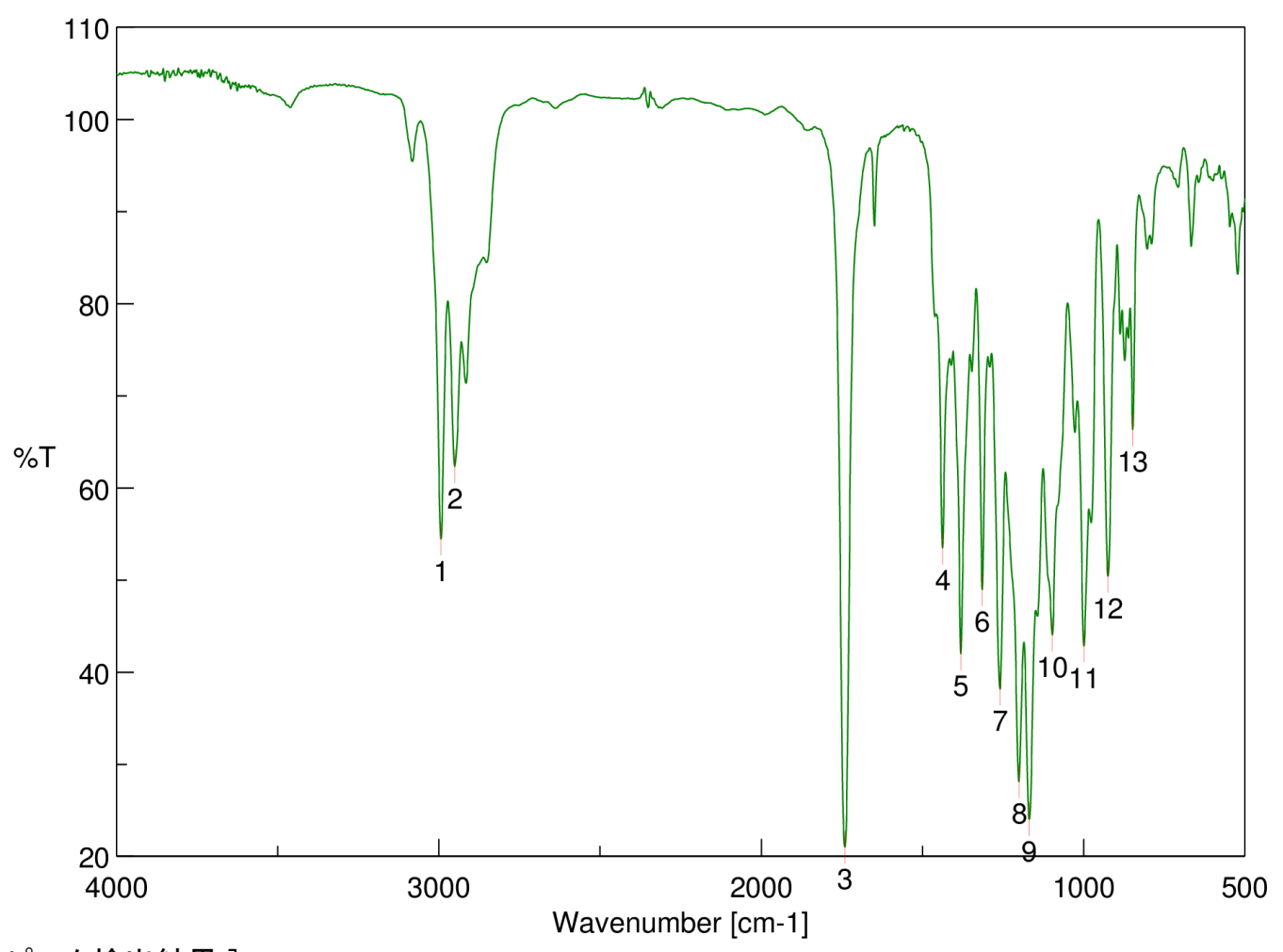

[ピーク検出結果 ]

$\begin{array}{llllll}\text { No. } & \text { 位置 } & \text { 強度 } & \text { No. } & \text { 位置 } & \text { 強度 } \\ 1 & 2993.94 & 54.4669 & 2 & 2950.55 & 62.3311 \\ 3 & 1740.44 & 21.0265 & 4 & 1437.67 & 53.4662 \\ 5 & 1380.78 & 41.9869 & 6 & 1314.25 & 48.9695 \\ 7 & 1259.29 & 38.1736 & 8 & 1200.47 & 28.1254 \\ 9 & 1168.65 & 24.002 & 10 & 1097.3 & 44.0488 \\ 11 & 998.946 & 42.8603 & 12 & 924.7 & 50.4298 \\ 13 & 847.561 & 66.3477 & & & \end{array}$<smiles>C=C[C@H]1C[C@@H](CC(C)=O)OC(C)(C)O1</smiles> 


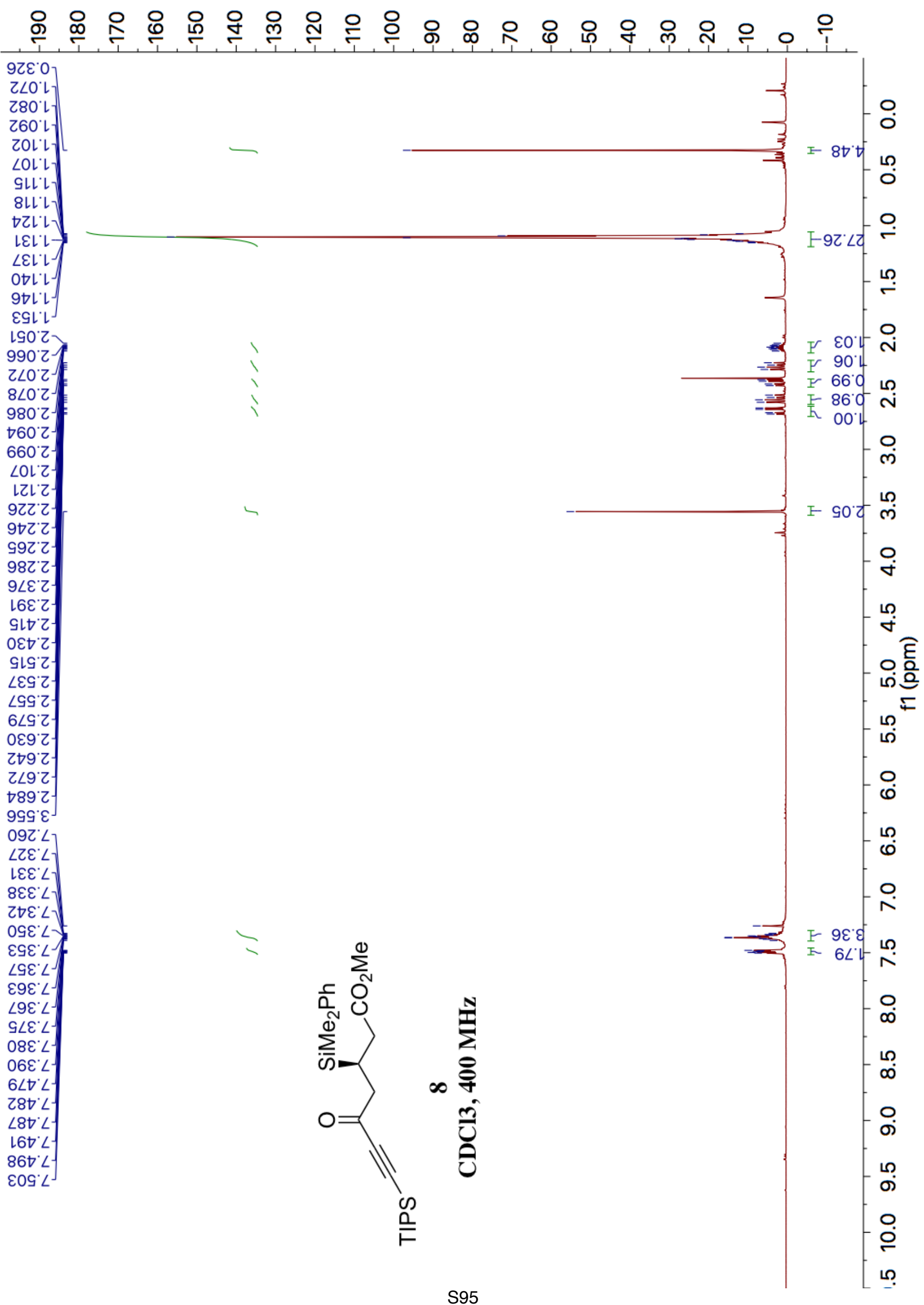




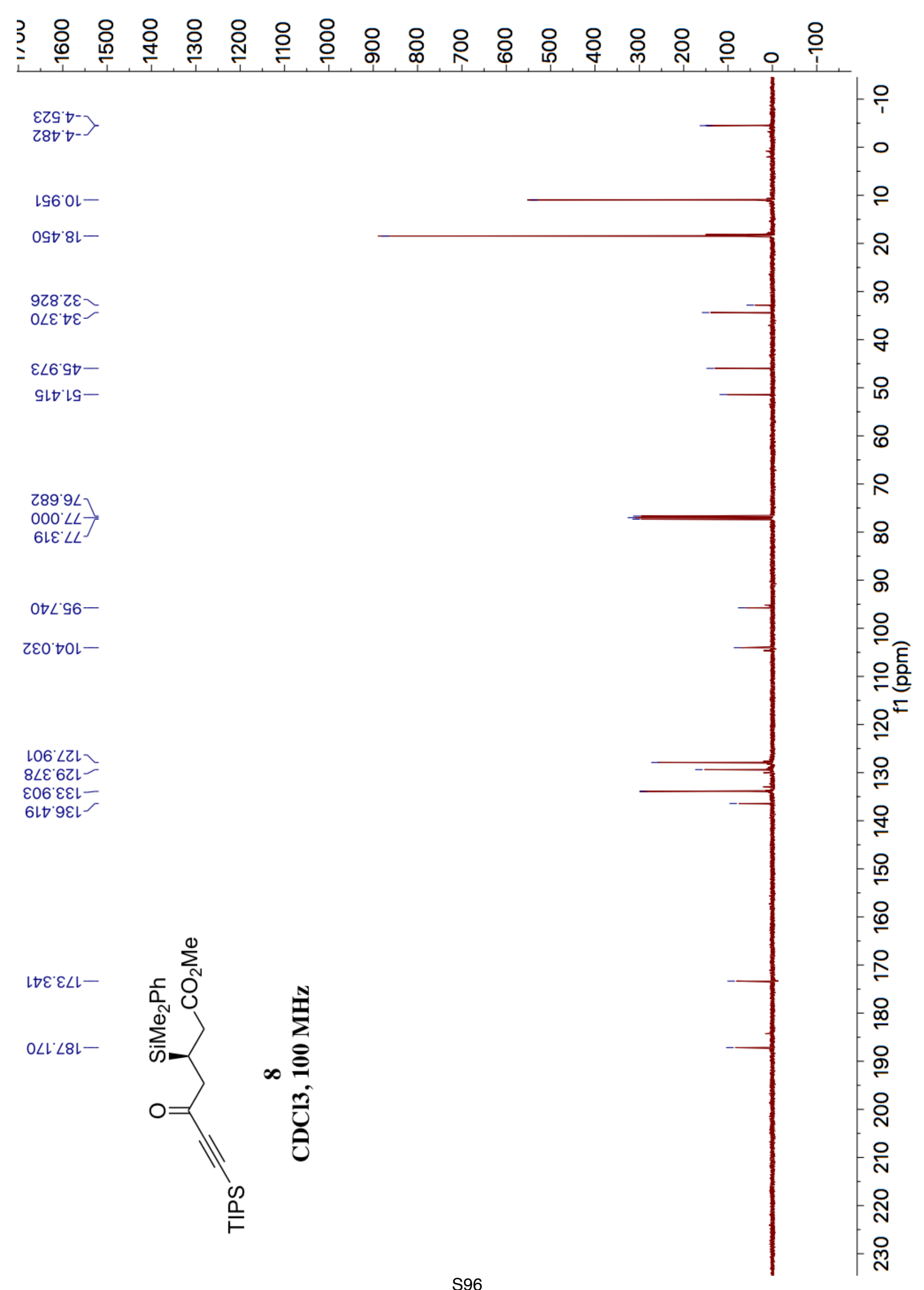




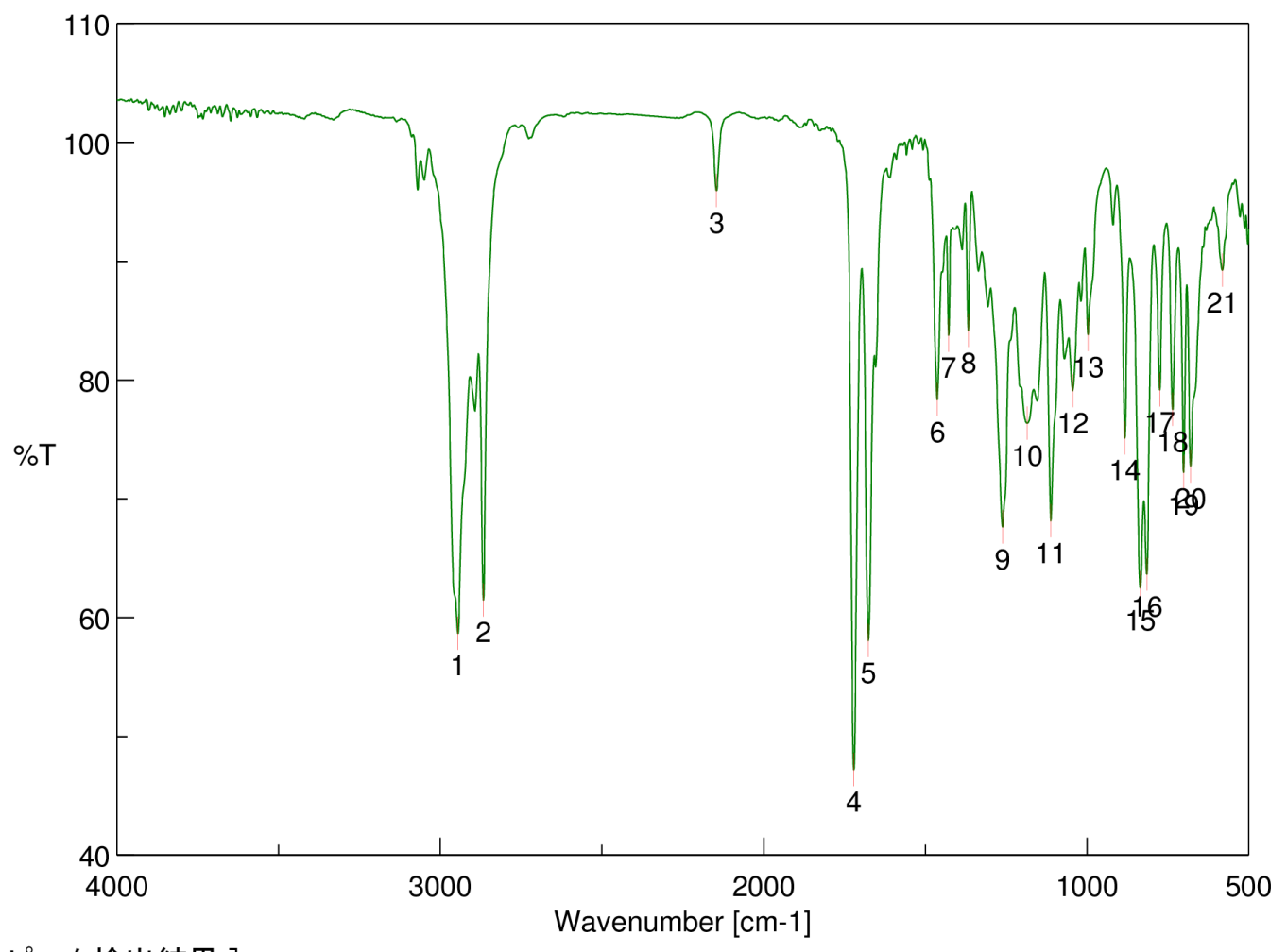

[ピーク検出結果 ]

$\begin{array}{llllll}\text { No. } & \text { 位置 } & \text { 強度 } & \text { No. } & \text { 位置 } & \text { 強度 } \\ 1 & 2945.73 & 58.6823 & 2 & 2866.67 & 61.4764 \\ 3 & 2146.38 & 95.9173 & 4 & 1721.16 & 47.1943 \\ 5 & 1675.84 & 58.0542 & 6 & 1463.71 & 78.3429 \\ 7 & 1428.03 & 83.7596 & 8 & 1367.28 & 84.1687 \\ 9 & 1261.22 & 67.6144 & 10 & 1185.04 & 76.3495 \\ 11 & 1111.76 & 68.1392 & 12 & 1044.26 & 79.1067 \\ 13 & 997.017 & 83.8152 & 14 & 883.238 & 75.1082 \\ 15 & 835.026 & 62.4803 & 16 & 814.777 & 63.6394 \\ 17 & 775.244 & 79.1549 & 18 & 735.71 & 77.5064 \\ 19 & 700.998 & 72.2065 & 20 & 679.785 & 72.7306\end{array}$

$\begin{array}{lll}21 & 581.433 & 89.2305\end{array}$<smiles>COC(=O)CC(CC(=O)C#CS[In])[AsH2]c1ccccc1</smiles> 


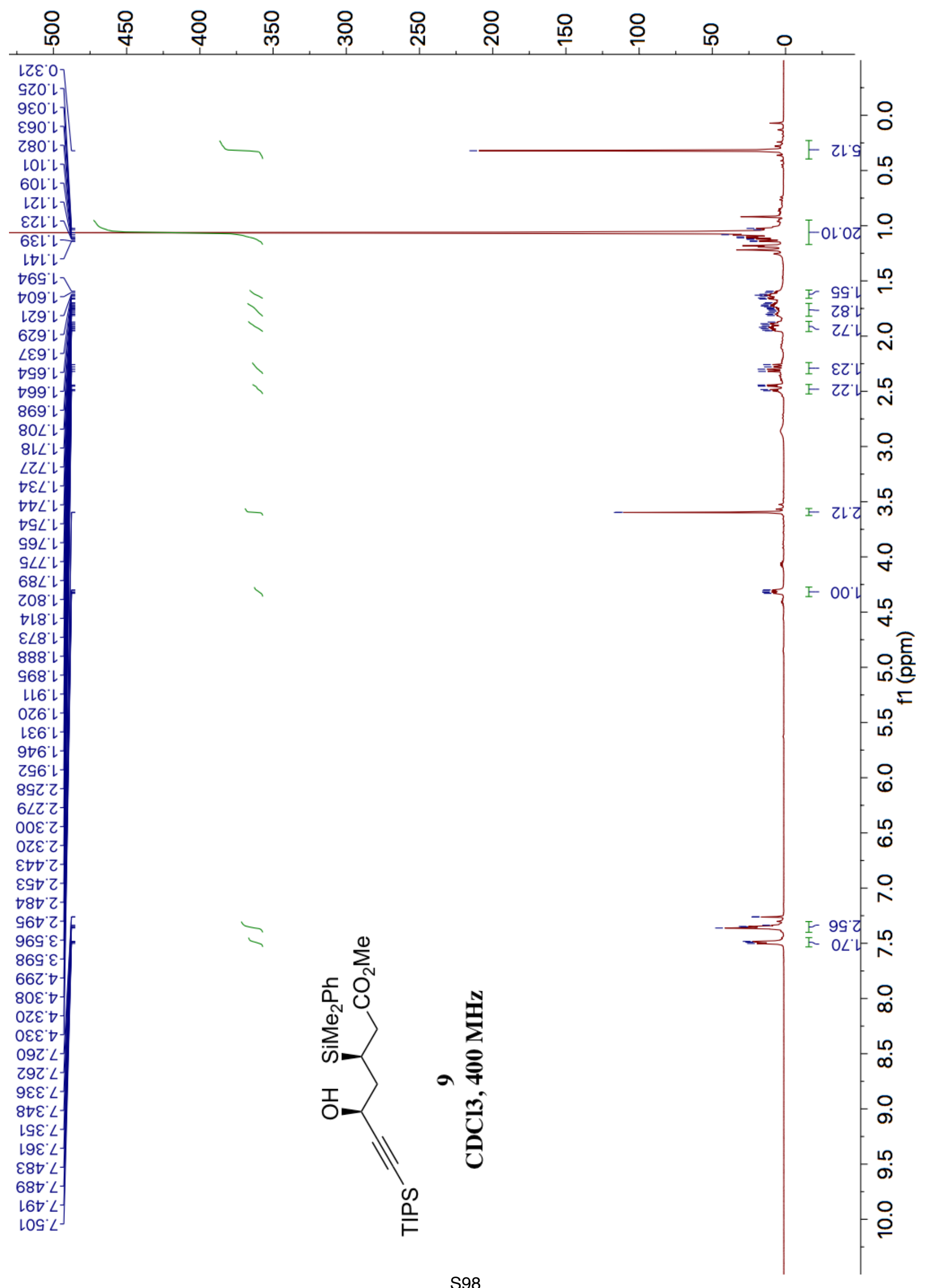




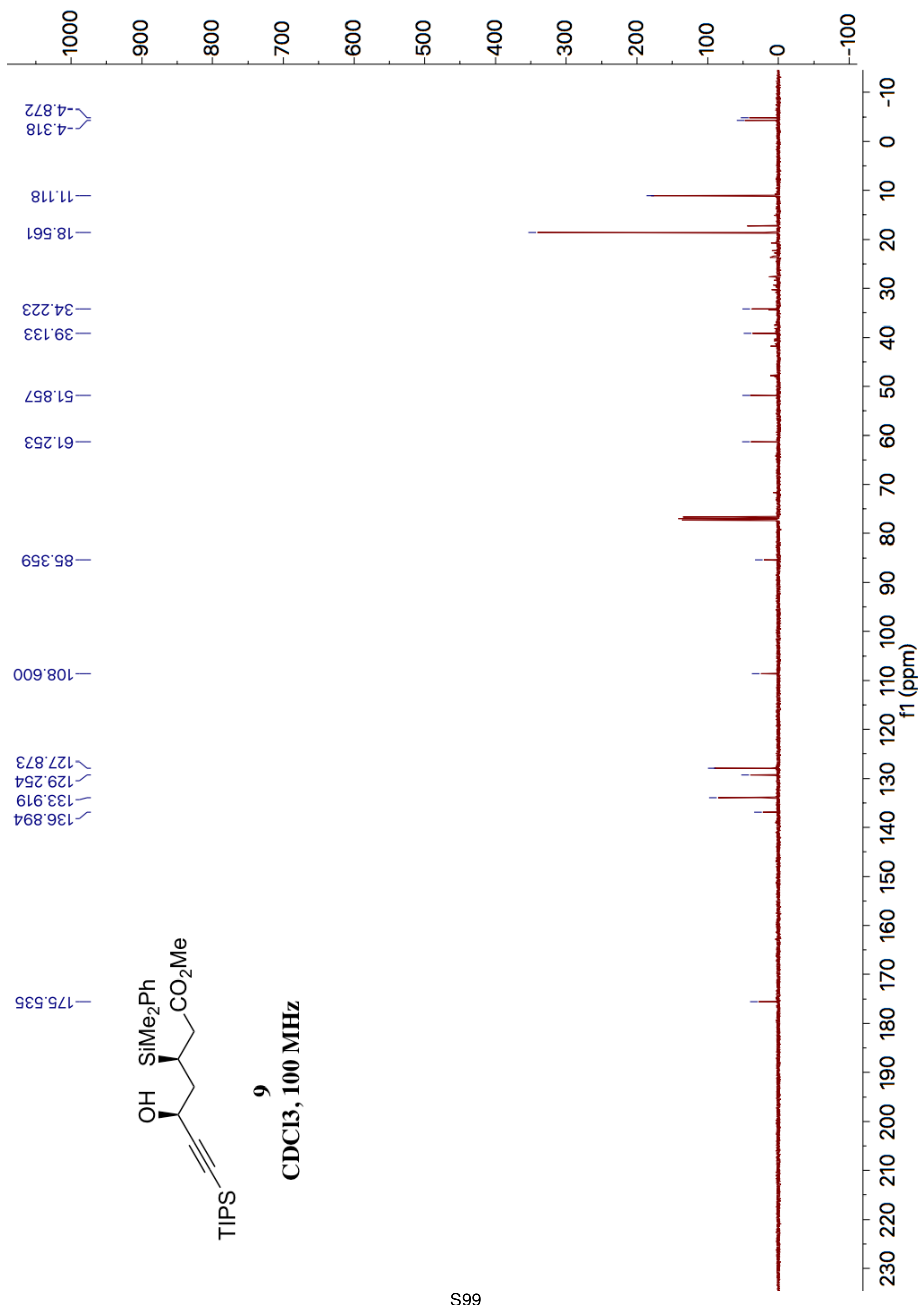




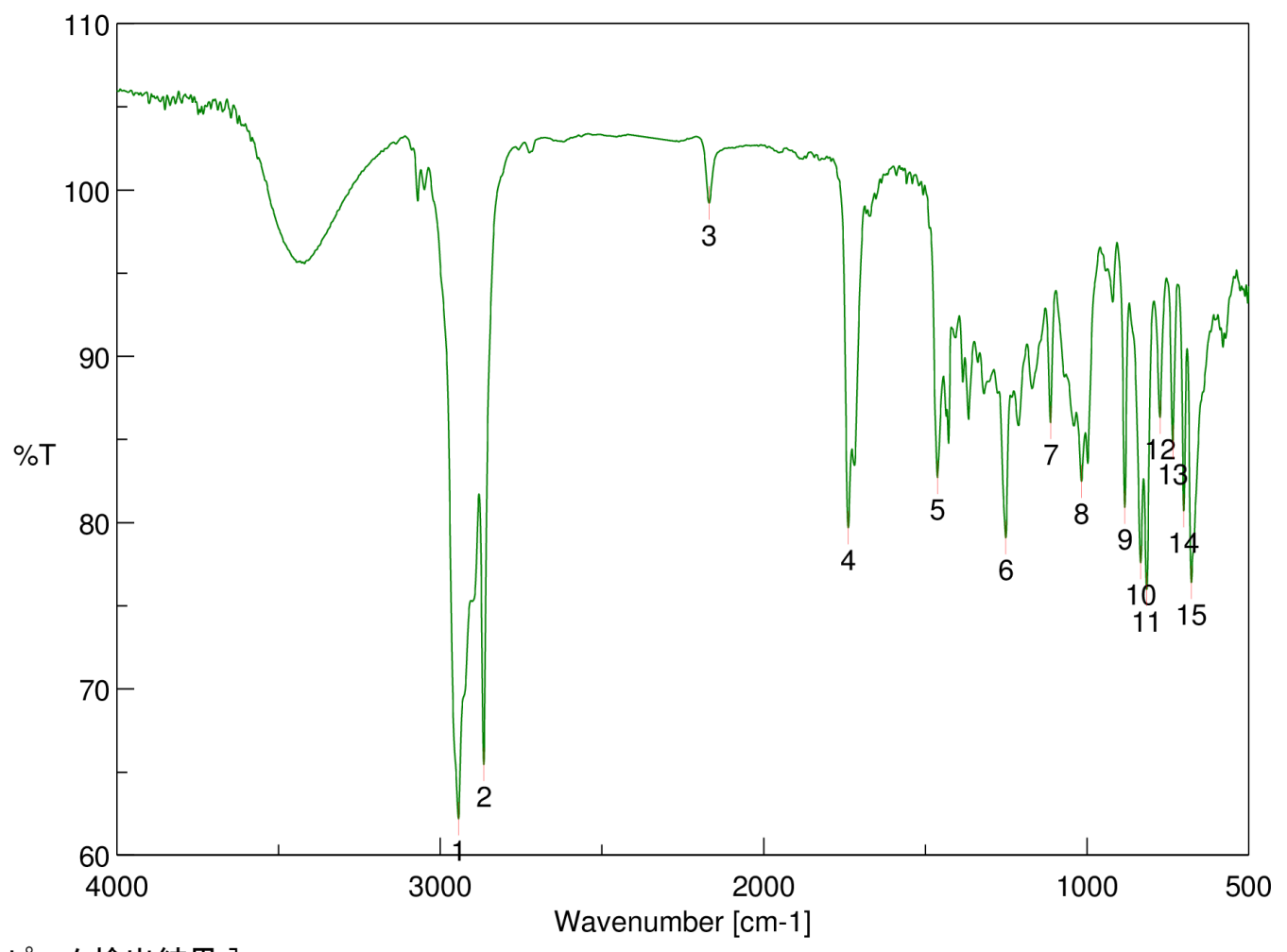

[ピーク検出結果 ]

$\begin{array}{llllll}\text { No. } & \text { 位置 } & \text { 強度 } & \text { No. } & \text { 位置 } & \text { 強度 } \\ 1 & 2943.8 & 62.1929 & 2 & 2865.7 & 65.4572 \\ 3 & 2168.56 & 99.2111 & 4 & 1738.51 & 79.6888 \\ 5 & 1462.74 & 82.6987 & 6 & 1251.58 & 79.0866 \\ 7 & 1112.73 & 86.0092 & 8 & 1017.27 & 82.4702 \\ 9 & 883.238 & 80.9175 & 10 & 834.062 & 77.581 \\ 11 & 815.742 & 75.9845 & 12 & 774.279 & 86.3283 \\ 13 & 734.746 & 84.8487 & 14 & 700.998 & 80.6979 \\ 15 & 677.856 & 76.3981 & & & \end{array}$

15 677.856 76.3981<smiles>CC(=O)CC(CC(O)C#C[PbH])C(C)(C)c1ccccc1</smiles> 


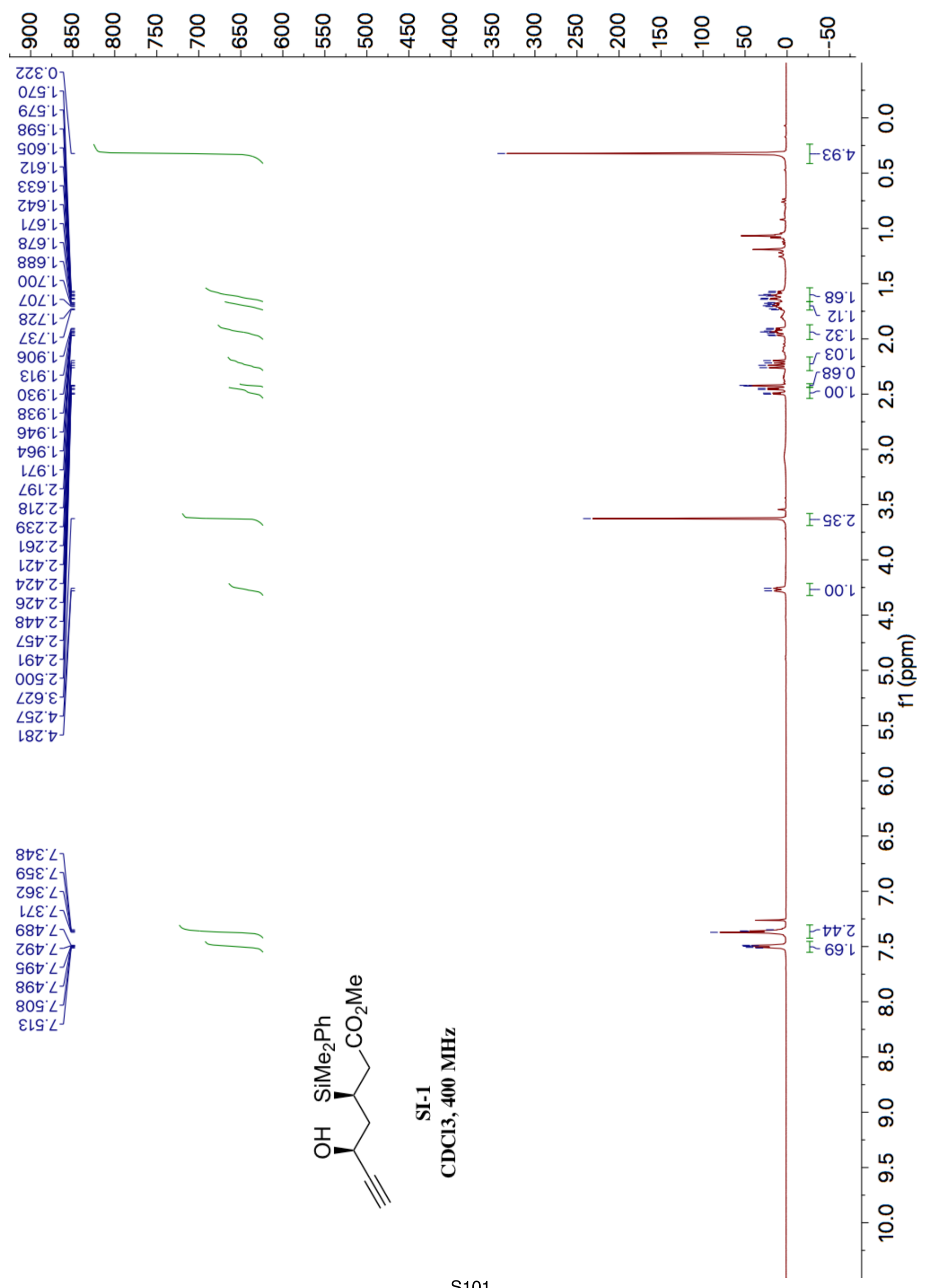




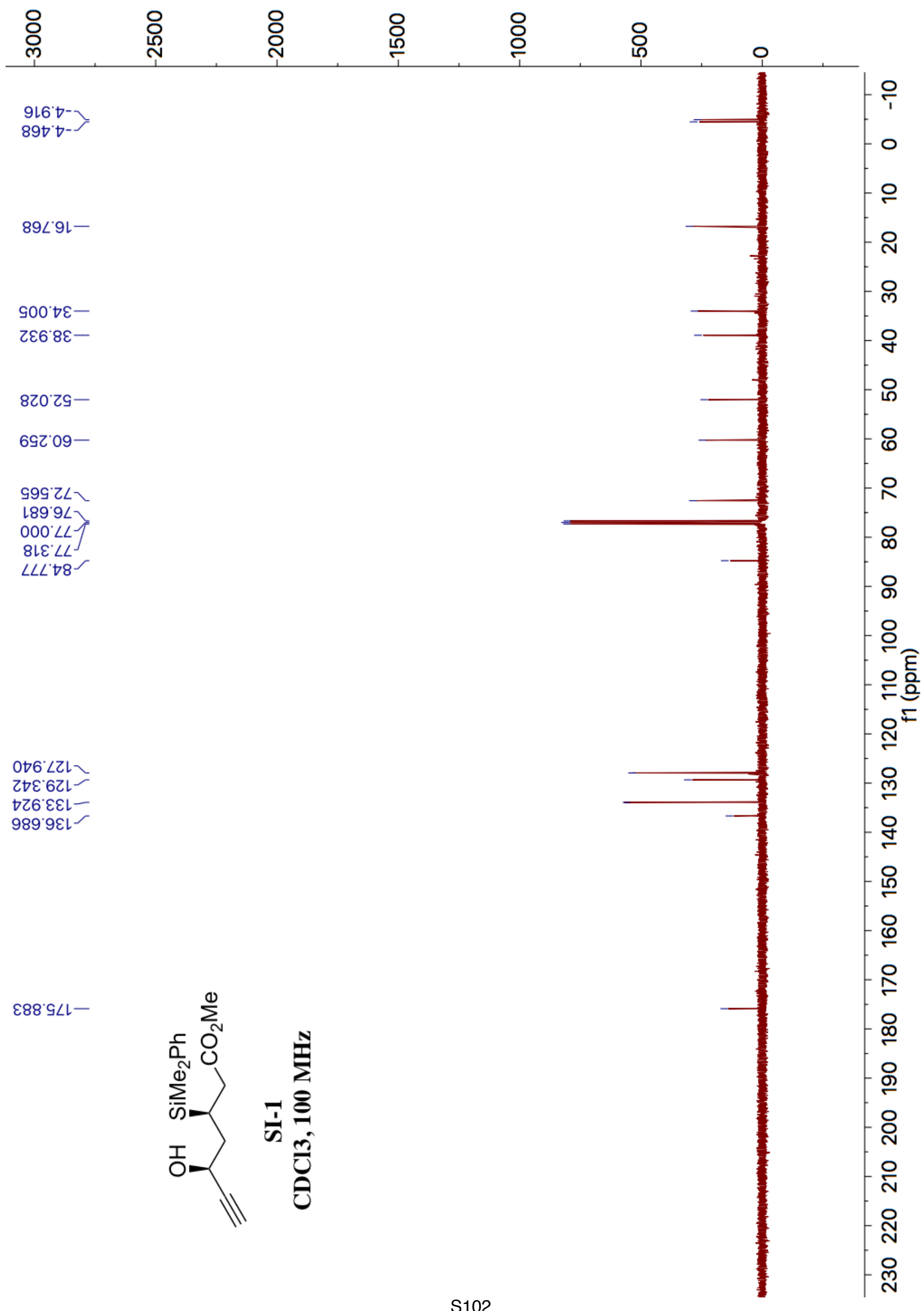




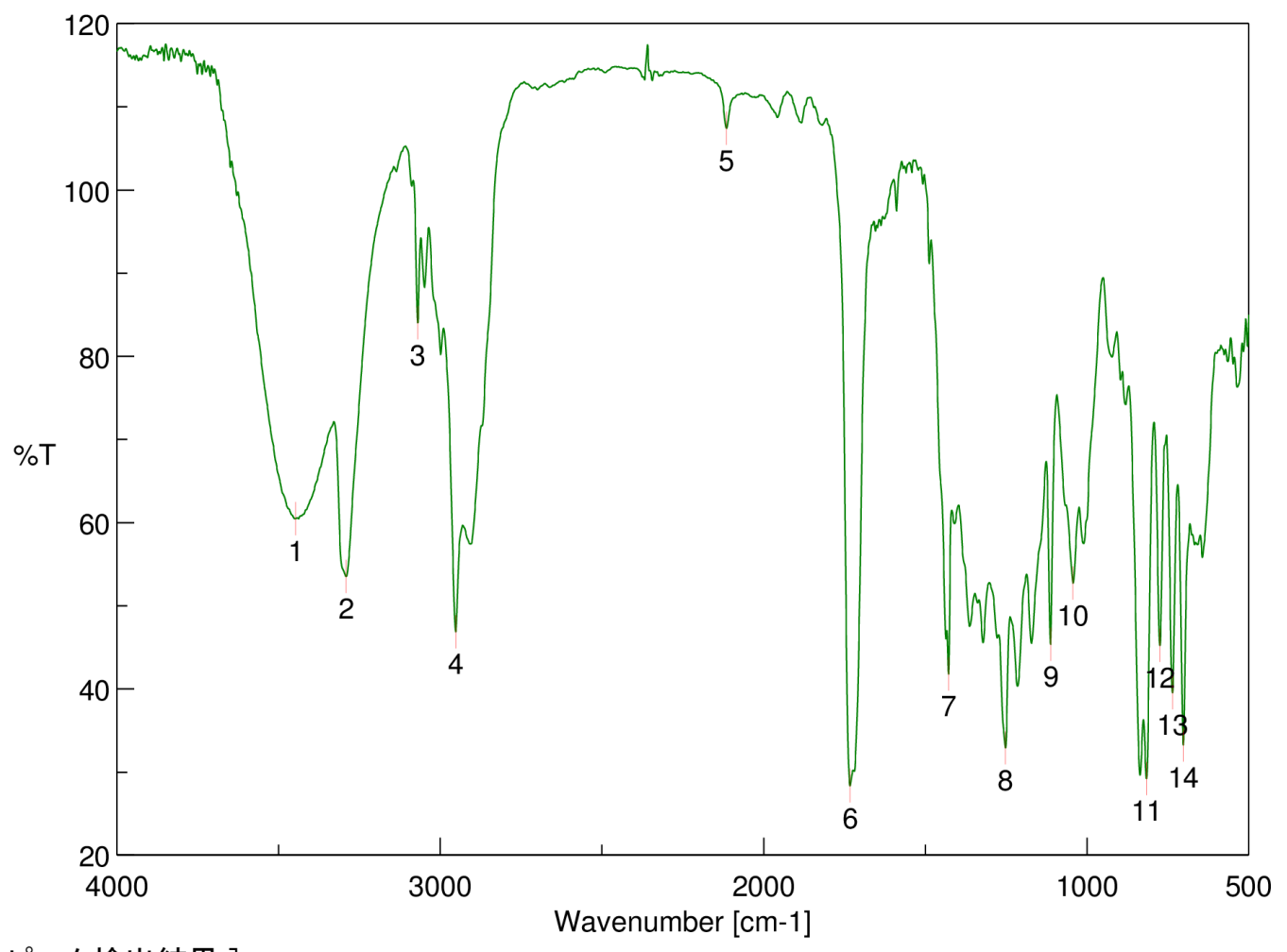

[ピーク検出結果 ]

$\begin{array}{llllll}\text { No. } & \text { 位置 } & \text { 強度 } & \text { No. } & \text { 位置 } & \text { 強度 } \\ 1 & 3448.1 & 60.4343 & 2 & 3290.93 & 53.482 \\ 3 & 3070.12 & 83.9826 & 4 & 2952.48 & 46.8548 \\ 5 & 2115.53 & 107.401 & 6 & 1732.73 & 28.3173 \\ 7 & 1428.03 & 41.7521 & 8 & 1252.54 & 32.9056 \\ 9 & 1112.73 & 45.3577 & 10 & 1043.3 & 52.6983 \\ 11 & 815.742 & 29.1785 & 12 & 775.244 & 45.2074 \\ 13 & 735.71 & 39.5002 & 14 & 701.962 & 33.2348\end{array}$<smiles>C#C[C@H](O)C[C@@H](CC(=O)OC)c1ccccc1</smiles> 


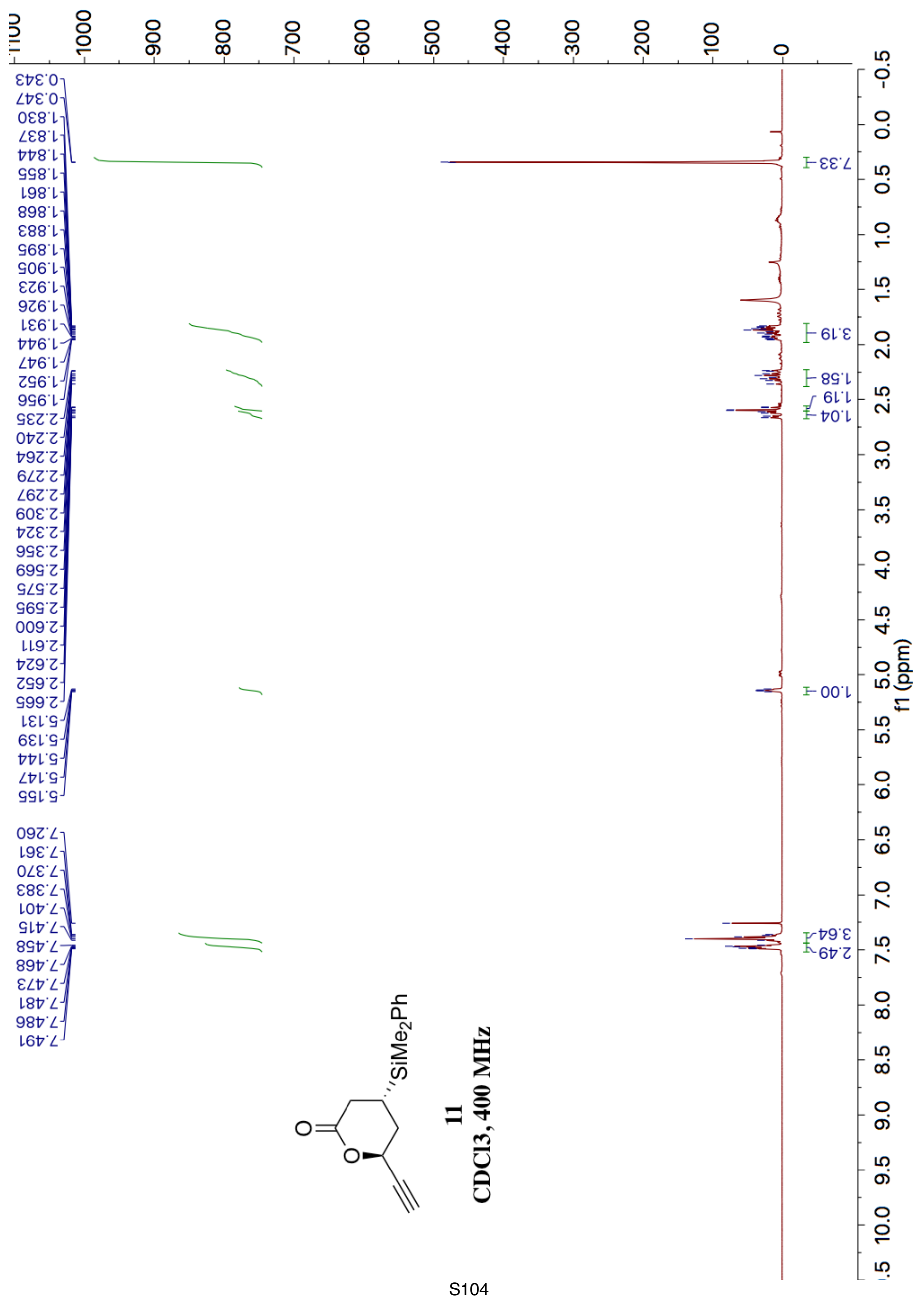




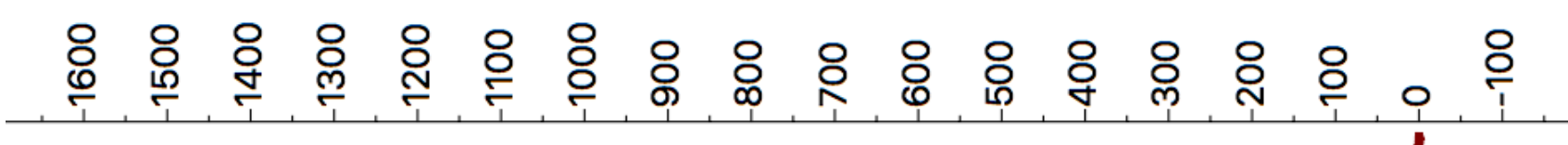

989'9-7

Z09'

SLE'GL-

0†Z:6乙

ZGS'0ะ

†6ع 69

ZZ9'9L

Z89 94

$000^{\circ} \angle L-$

$6 \mathrm{~L} \varepsilon^{\prime} \angle L$

$\angle \nabla Z 08$

†七l:8ZL

69L'6ZL-

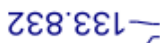

9Gl'GEL

$996.691-$
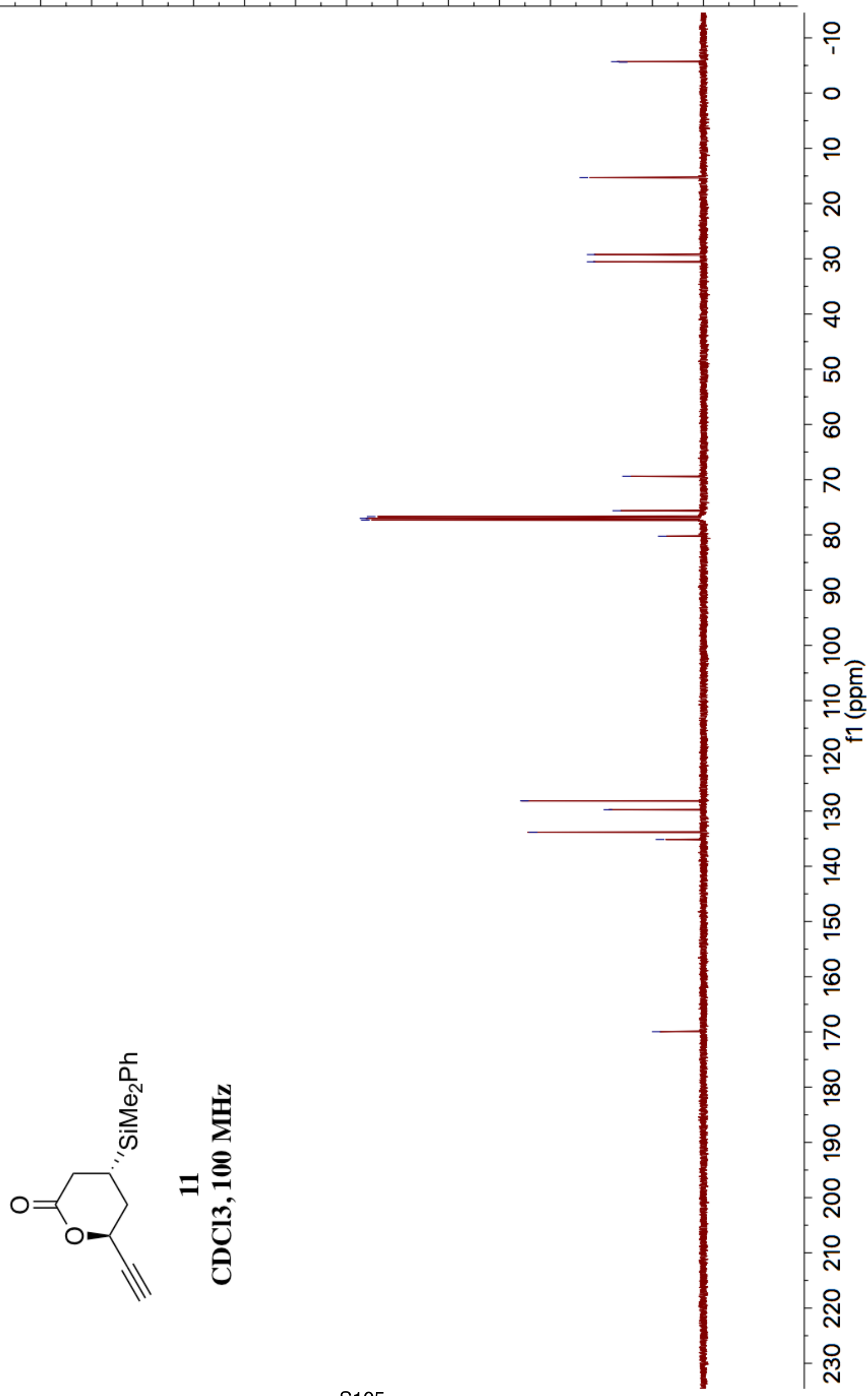


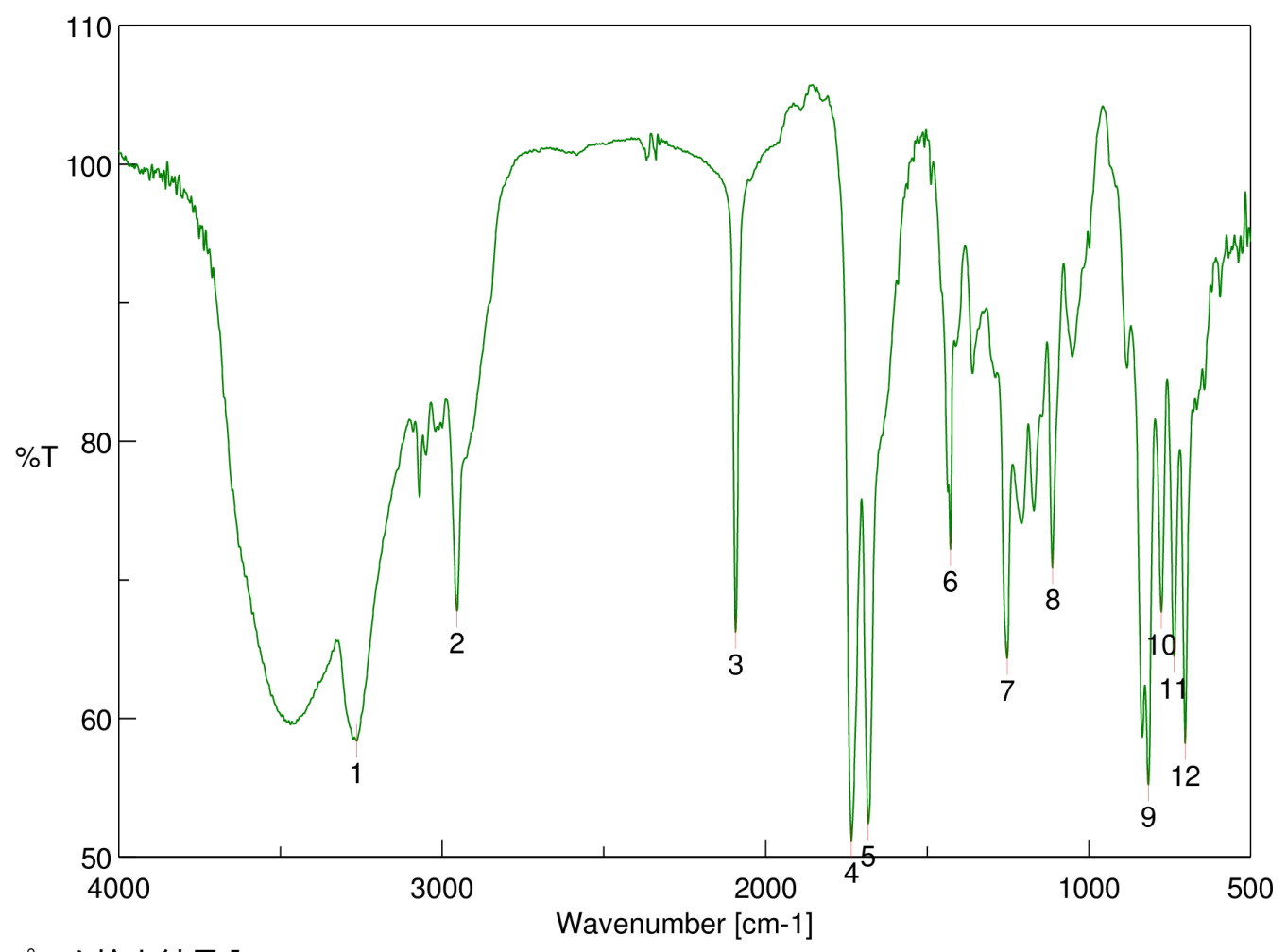

[ピーク検出結果 ]

$\begin{array}{llllll}\text { No. } & \text { 位置 } & \text { 強度 } & \text { No. } & \text { 位置 } & \text { 強度 } \\ 1 & 3264.89 & 58.3741 & 2 & 2954.41 & 67.7418 \\ 3 & 2092.39 & 66.2368 & 4 & 1734.66 & 51.1806 \\ 5 & 1682.59 & 52.3871 & 6 & 1428.03 & 72.2198 \\ 7 & 1252.54 & 64.3294 & 8 & 1112.73 & 70.8925 \\ 9 & 815.742 & 55.2062 & 10 & 776.208 & 67.6755 \\ 11 & 736.674 & 64.471 & 12 & 701.962 & 58.1863\end{array}$<smiles>C#C[C@H]1C[C@H](S(C)(C)c2ccccc2)CC(=O)O1</smiles> 


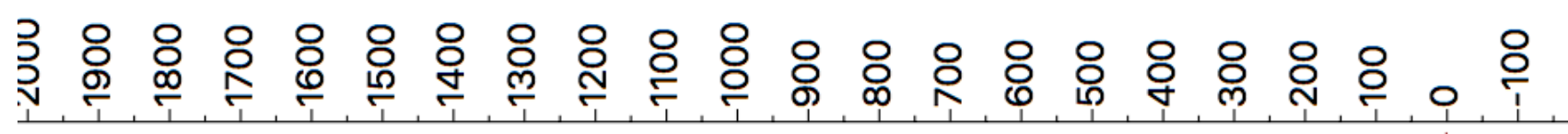

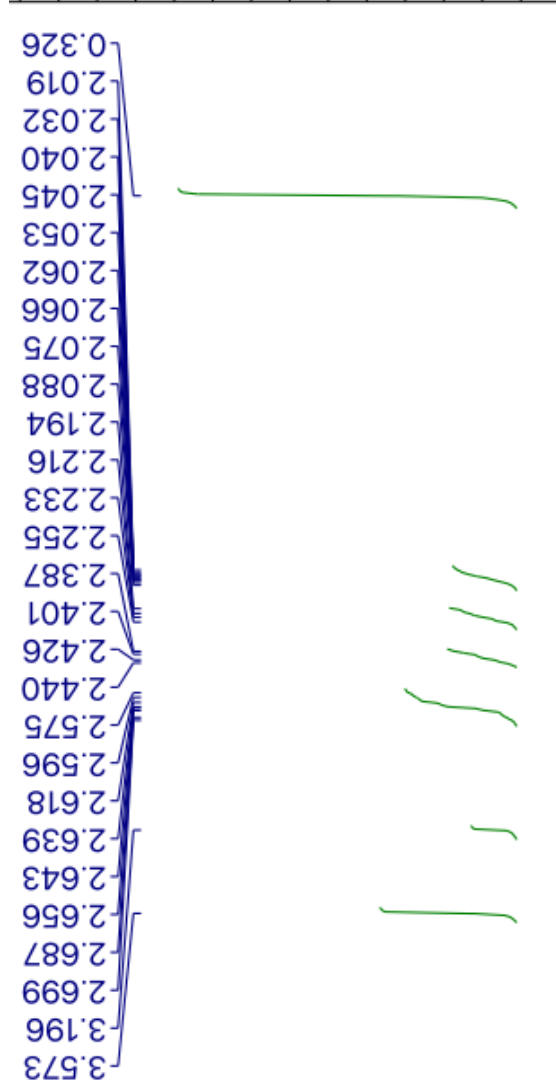

092 $\angle$

OGE $\angle$

GSE $\angle-$

เ9E $\angle$

G9 $\varepsilon^{\circ} \angle$

$99 \varepsilon^{\circ} \angle$

$89 \varepsilon<1$

$G \angle \varepsilon^{\prime} \angle$

$\left.6 \angle \varepsilon^{\circ} \angle\right]$

$98 \varepsilon^{\circ} \angle \sqrt{ }$

$68 \varepsilon^{\circ} \angle$

乙6E $\angle J$

$96 \varepsilon^{\circ} \angle$

$08 \nabla^{\circ} \angle$

Z8० $\angle$

$\angle 8 \nabla^{\circ} \angle$

Z6t $\angle$

$66 \nabla^{\circ} L$

$\nabla 09^{\circ} \angle$

LIS. $L$
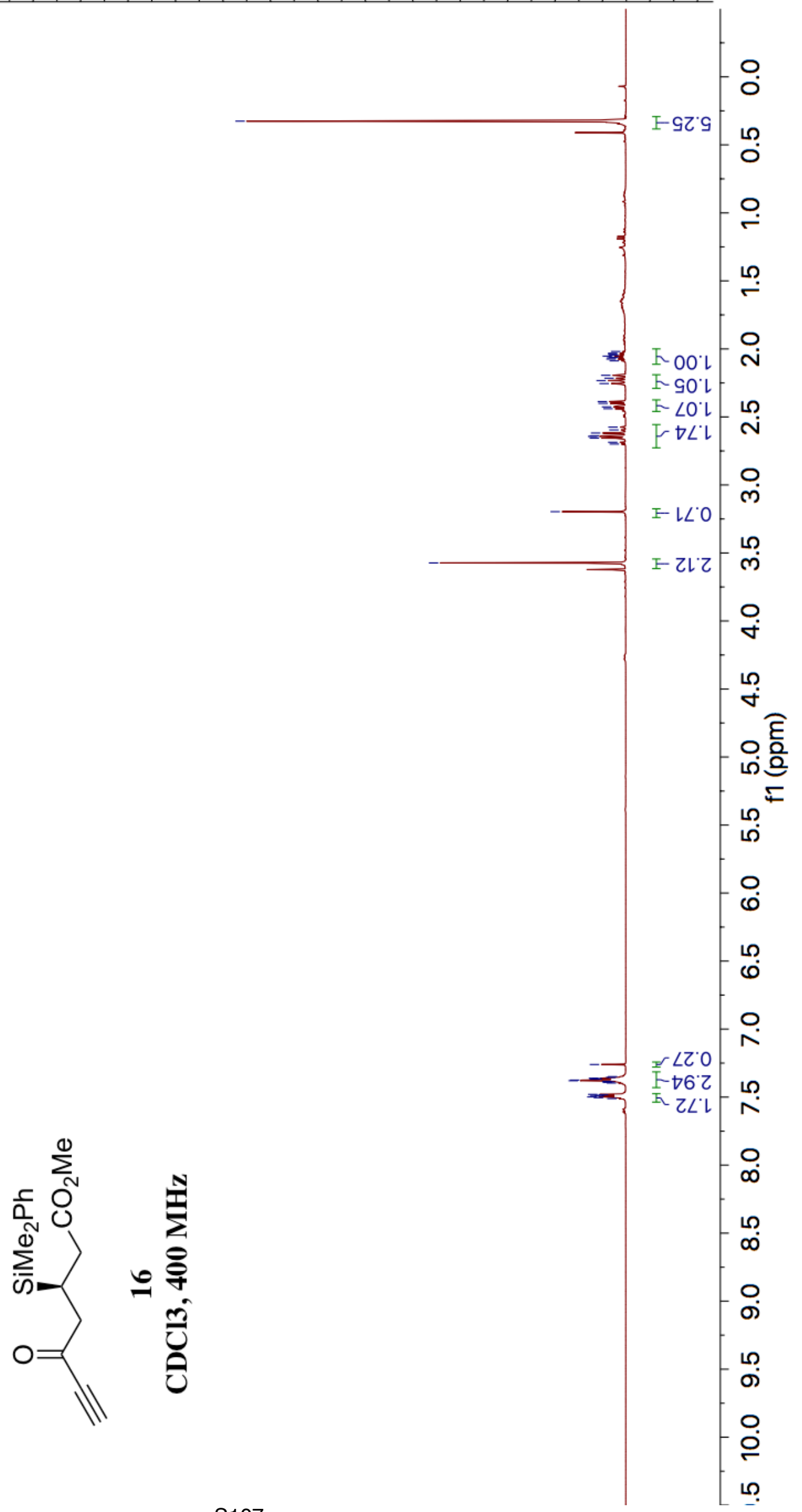
年 운 운

จ.9 $\nabla^{-}$
$899^{\circ} \nabla^{-}$

○ฤで

8LL'SD-

†SG'LS-

Z89.9L

$000 \angle L$

$61 \varepsilon^{\prime} \angle L J$

$88 \mathrm{~S}^{\circ} 8 \mathrm{~L}^{\circ}$

ZLE'เ8

E96 $\mathrm{LZL}$

GLt $6 Z L-$

จเ6.عEL-

9เ乙'9عᄂ

$9 \varepsilon 9^{\prime} \varepsilon \angle L-$

$\varepsilon \angle L \cdot 981-$
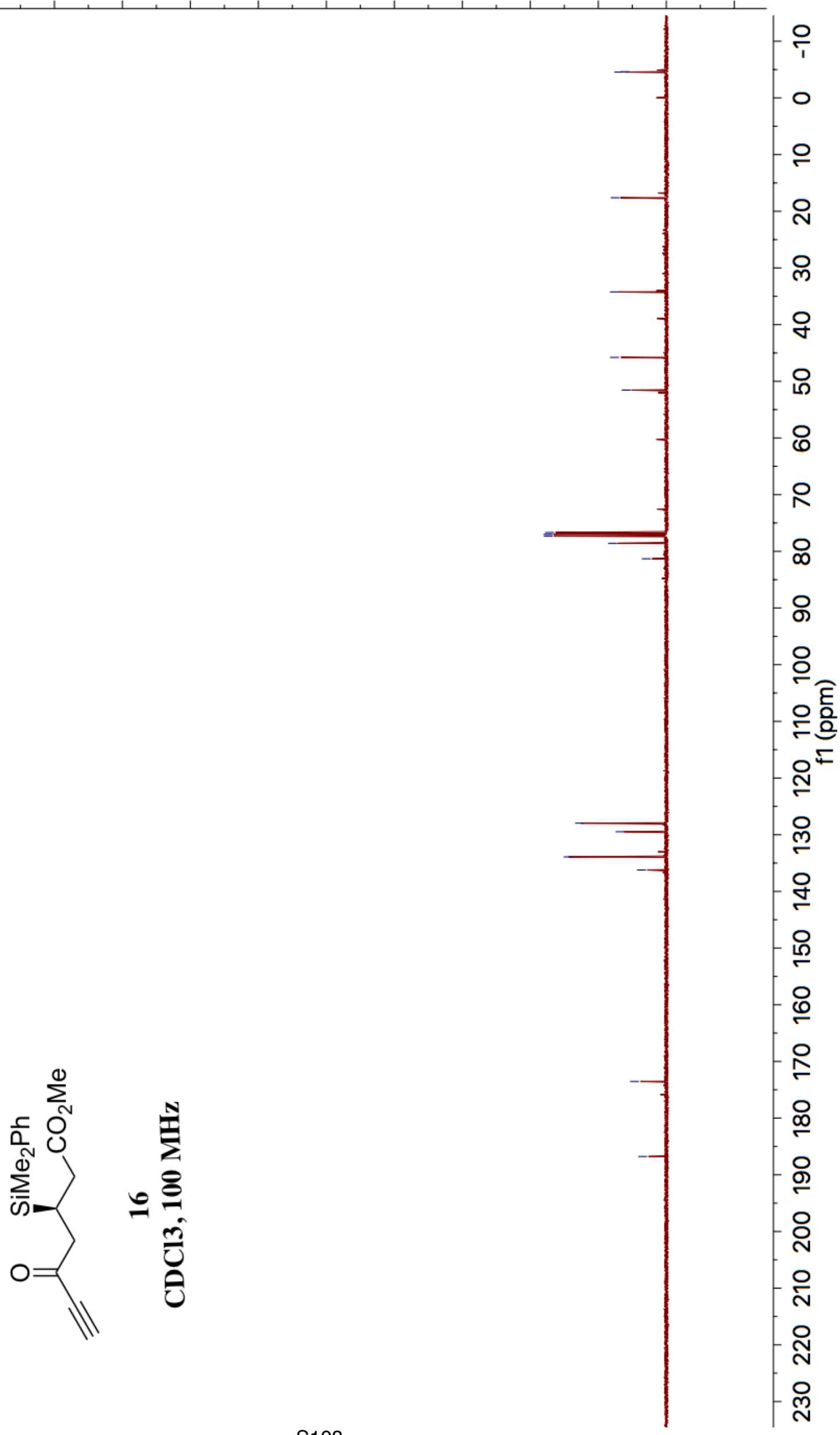


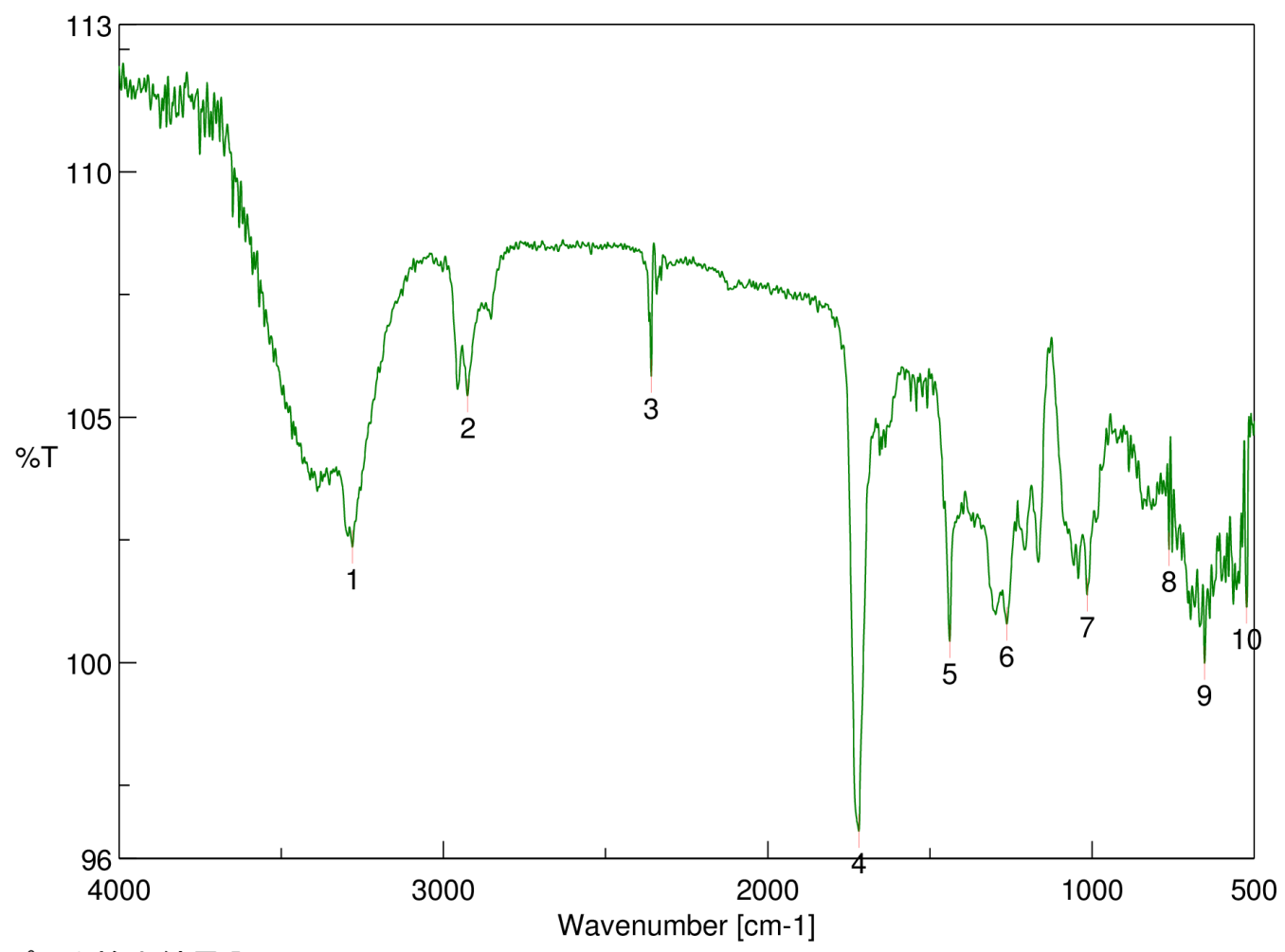

[ピーク検出結果 ]

$\begin{array}{llllll}\text { No. } & \text { 位置 } & \text { 強度 } & \text { No. } & \text { 位置 } & \text { 強度 } \\ 1 & 3280.32 & 102.35 & 2 & 2925.48 & 105.435 \\ 3 & 2359.48 & 105.835 & 4 & 1719.23 & 96.5608 \\ 5 & 1438.64 & 100.429 & 6 & 1263.15 & 100.784 \\ 7 & 1015.34 & 101.38 & 8 & 762.709 & 102.304 \\ 9 & 652.786 & 99.9851 & 10 & 523.579 & 101.126\end{array}$<smiles>C#CC(=O)C[C@H](CC(=O)OC)c1ccccc1</smiles> 


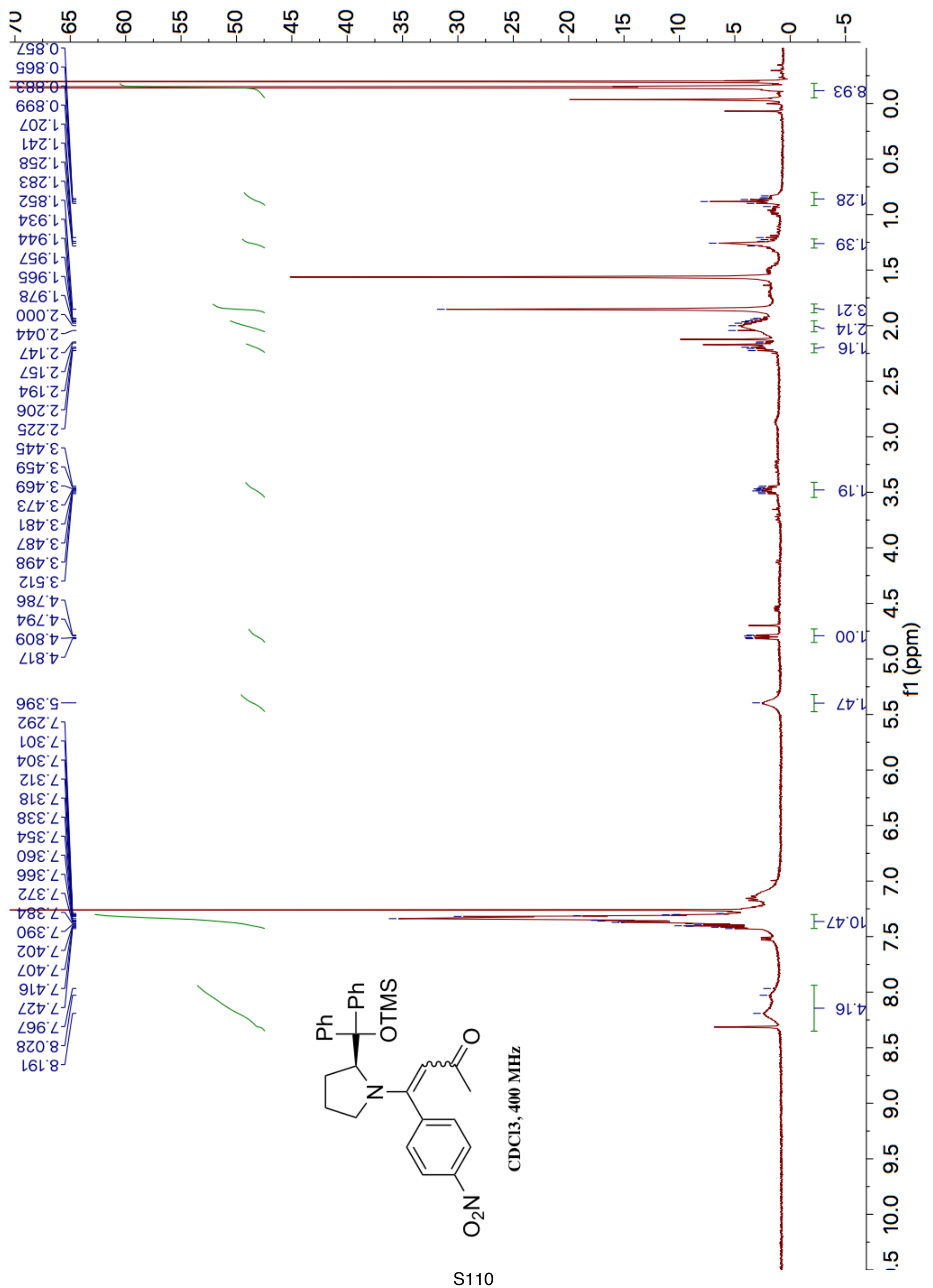




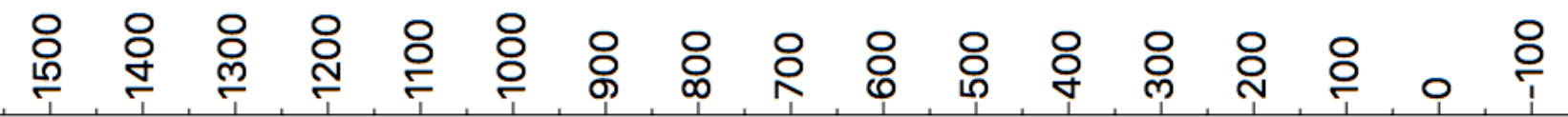

$880^{\circ} Z-$

9ZL'ZZ-

$\angle L \varepsilon^{\circ} \angle Z-$

$18 \angle \cdot 0 \varepsilon-$

$6 \angle 6^{\circ} \varepsilon \triangleright-$

ᄂ०O'99-

$289{ }^{\circ} /$

$000 \angle L\}$

$91 \mathcal{C}^{\circ} \varepsilon$

$\varepsilon 97^{\circ} \angle Z L$

$\nabla 69^{\circ} \angle Z L$

$\varepsilon \angle L \angle Z L$

$80 L^{\circ} 8 \mathrm{LL}$

lLZ'6ZL-

เฤย'6ZL

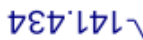

960でレフ

LOE'Z७L

$\varepsilon 9 Z \angle \nabla l$

عl8.89l-

Z66'29l-
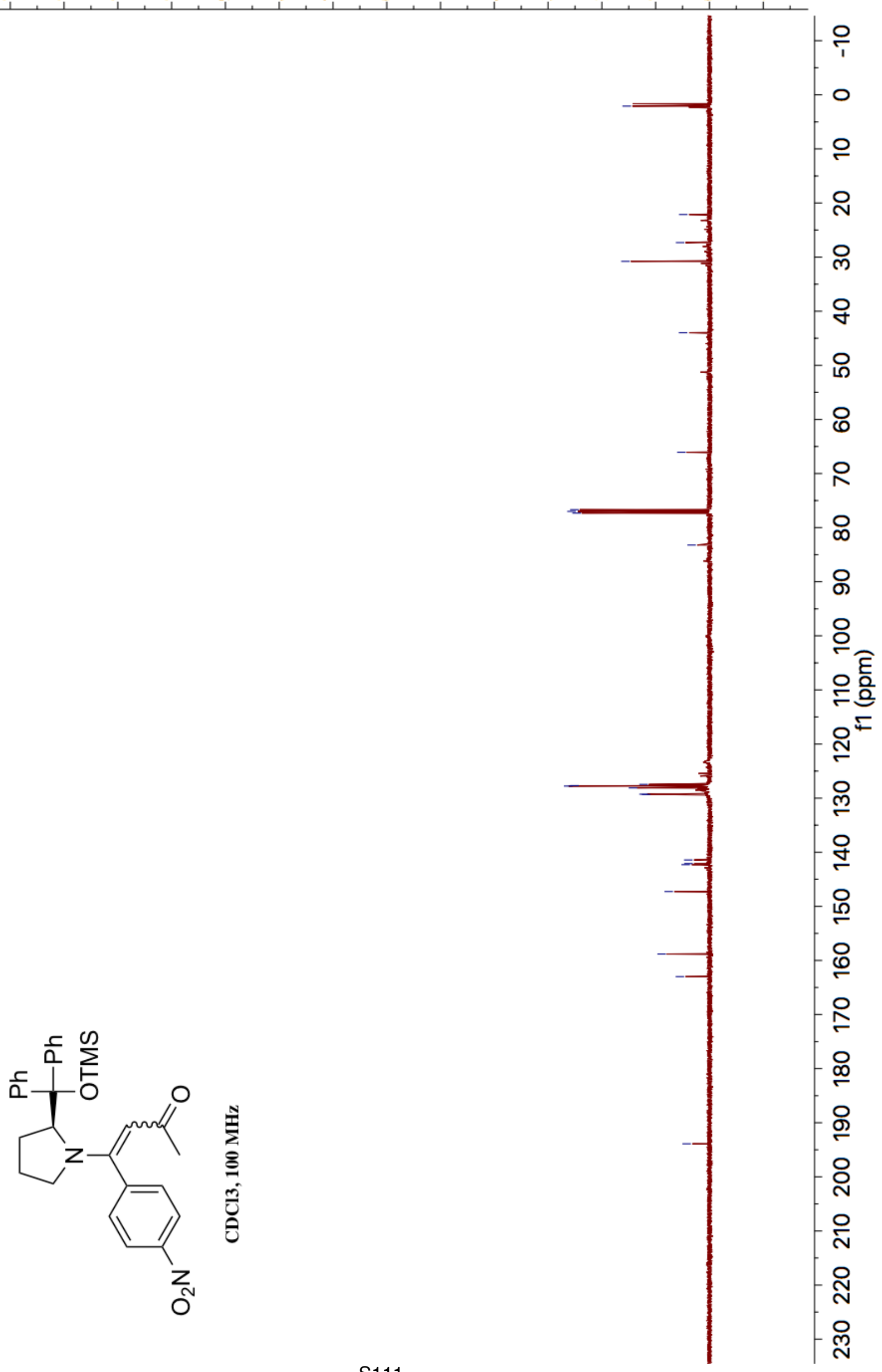


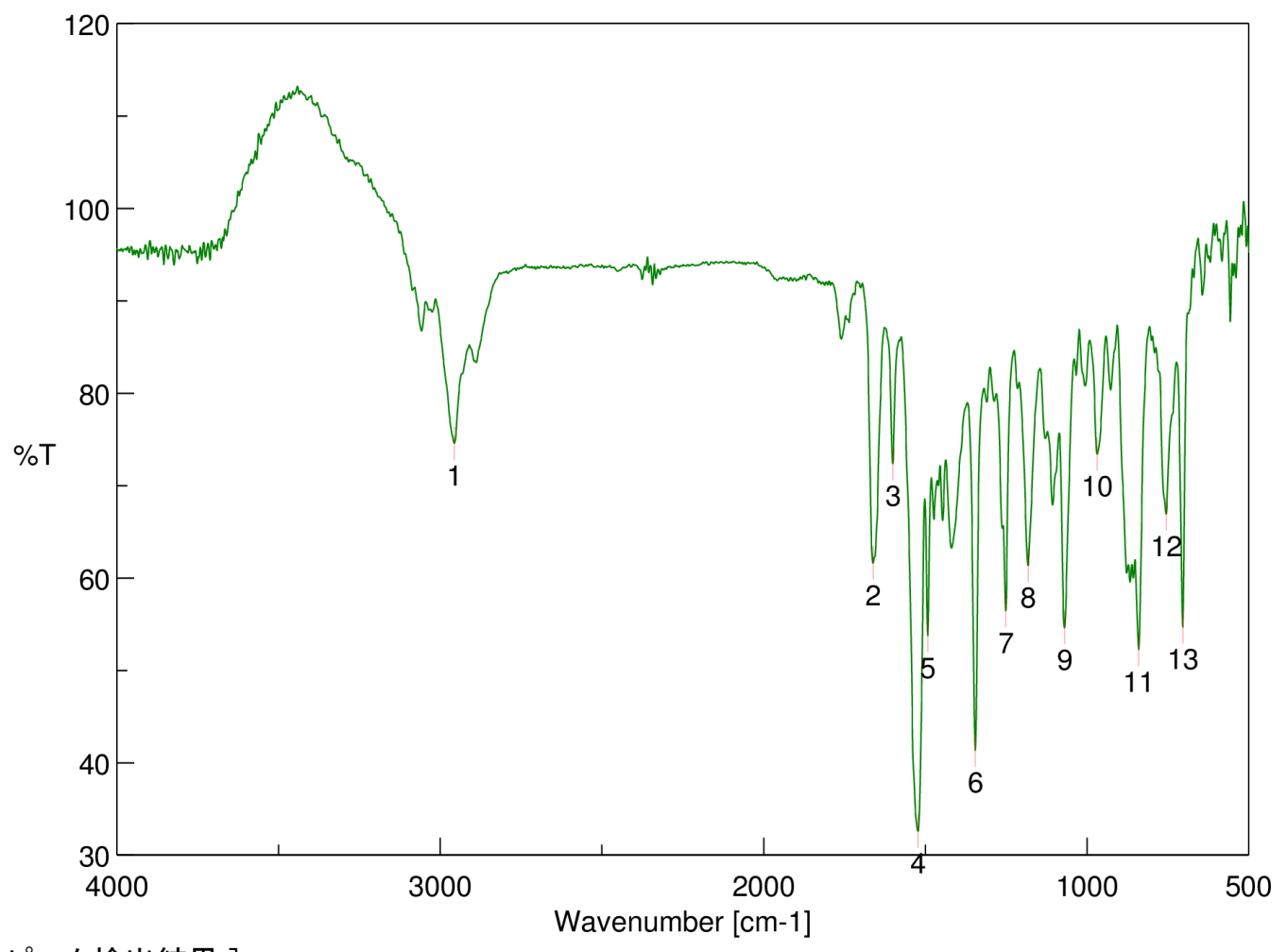

[ピーク検出結果 ]

$\begin{array}{llllll}\text { No. } & \text { 位置 } & \text { 強度 } & \text { No. } & \text { 位置 } & \text { 強度 } \\ 1 & 2956.34 & 74.5878 & 2 & 1661.37 & 61.612 \\ 3 & 1600.63 & 72.3715 & 4 & 1522.52 & 32.5978 \\ 5 & 1492.63 & 53.7373 & 6 & 1345.11 & 41.365 \\ 7 & 1251.58 & 56.4595 & 8 & 1182.15 & 61.3301 \\ 9 & 1069.33 & 54.6235 & 10 & 969.055 & 73.3985 \\ 11 & 839.847 & 52.2597 & 12 & 754.995 & 66.9187 \\ 13 & 703.89 & 54.6881 & & & \end{array}$

$13 \quad 703.89 \quad 54.6881$

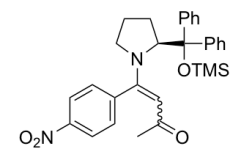




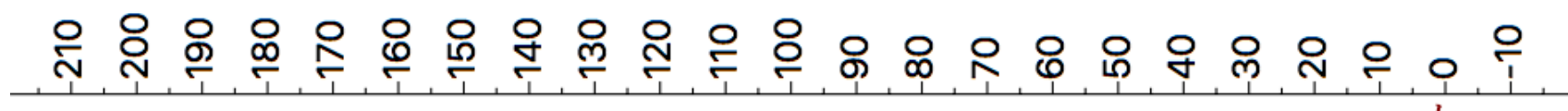

$\nabla \varepsilon 8^{\circ} 0$

$088^{\circ} 0$

OLOL

ZZO'L

ZEO' $\angle 90^{\circ}$

$\angle L O^{\circ} \mathrm{L}$

$\varepsilon 60^{\circ} \mathrm{L}$

GOL'L

OZL'L

$9 \varepsilon L$ L

EGL:

$\nabla 9 L^{\circ} L$

$0 \angle L L$

9LL'

98LL-

$\nabla 6 L^{\circ} L$

002 '

80Z L-

ZZZ L

乙E乙 'L

乙๖乙 レ.

9GZ'

$\rightarrow 8 Z^{\circ}$

8हย' 9

$9 \angle \varepsilon^{\prime} 9^{\gamma}$

096.9

$0 \angle 6^{\circ} 9$

<6 6 : 9

$09 \varepsilon^{\circ} L$

GGE $L$

$69 \varepsilon^{\circ} \angle$

$\neg 9 \varepsilon^{\circ} \angle$

๑8 $\varepsilon^{\circ} \angle$

$66 \varepsilon^{\circ}\llcorner$

$90 t^{\circ} L$

$96 \nabla^{\circ} L$

$66 \nabla^{\circ} L$

OLG $L$

SIS: $\angle$

6LS'L

GE9 $L$

$\nabla \nabla 9^{\circ} \angle$

Z99 $\mathrm{L}$

$199^{\circ} \mathrm{L}$

$\nabla \angle 9^{\circ} \angle$

$669^{\circ} \mathrm{L}$

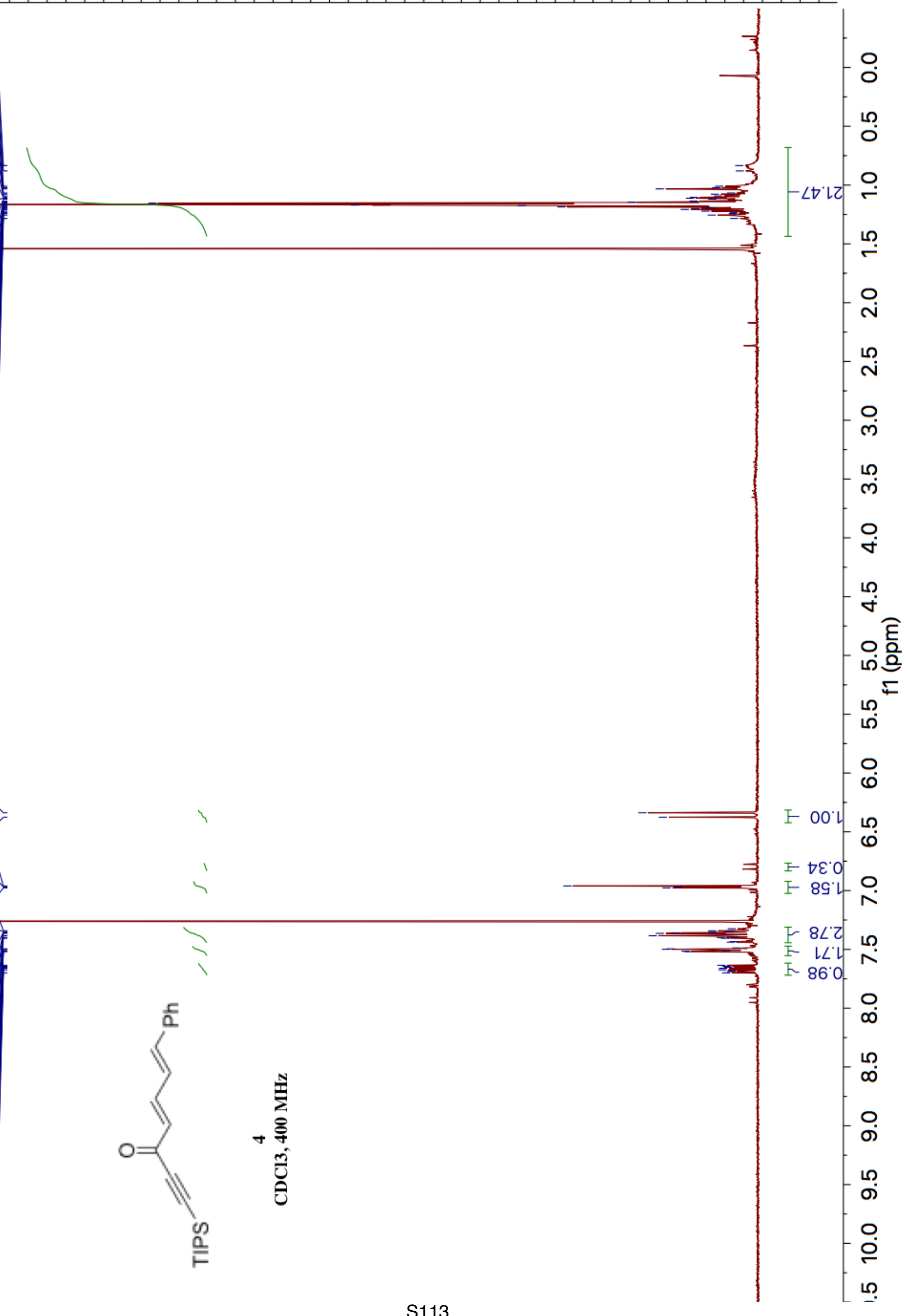




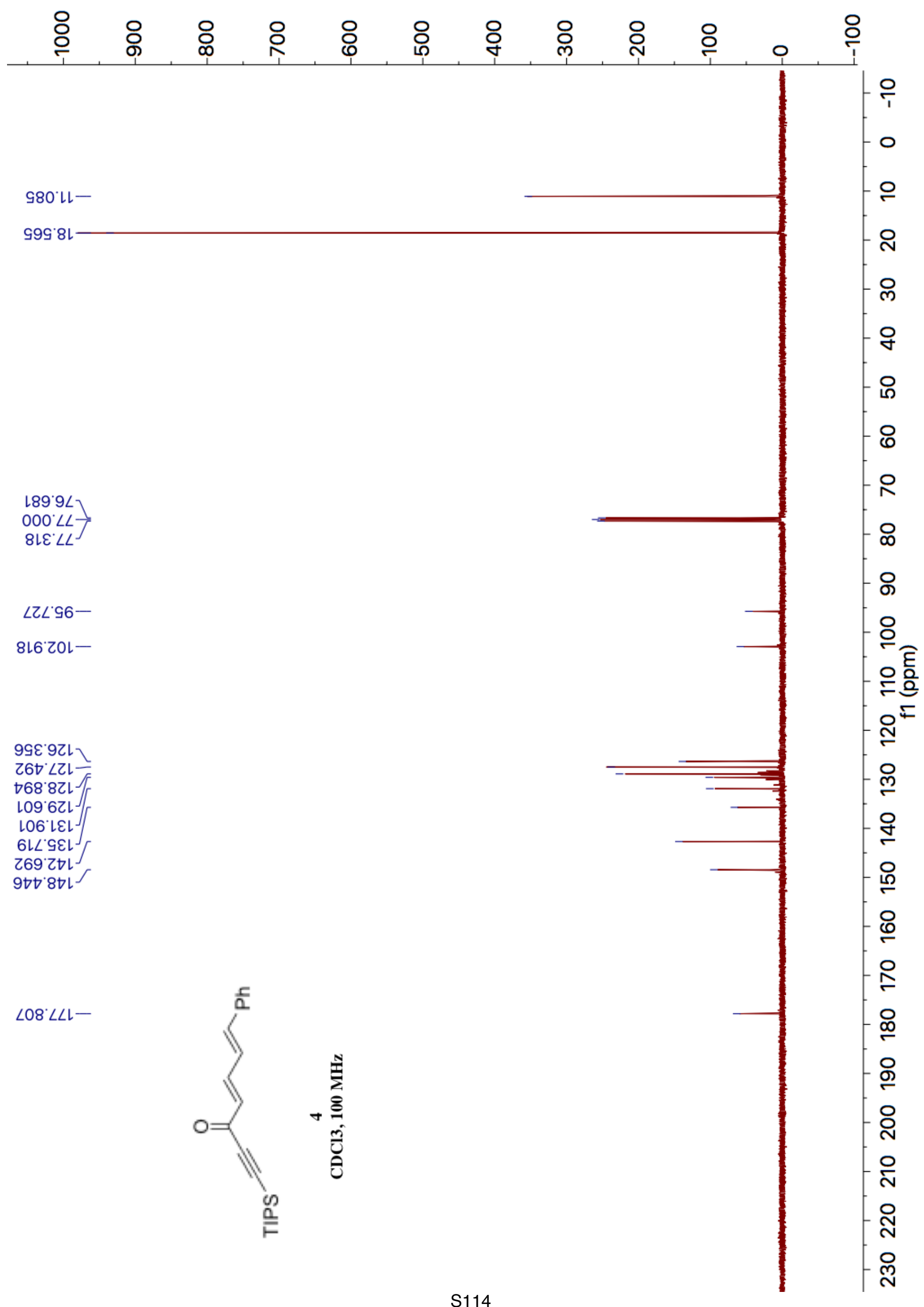




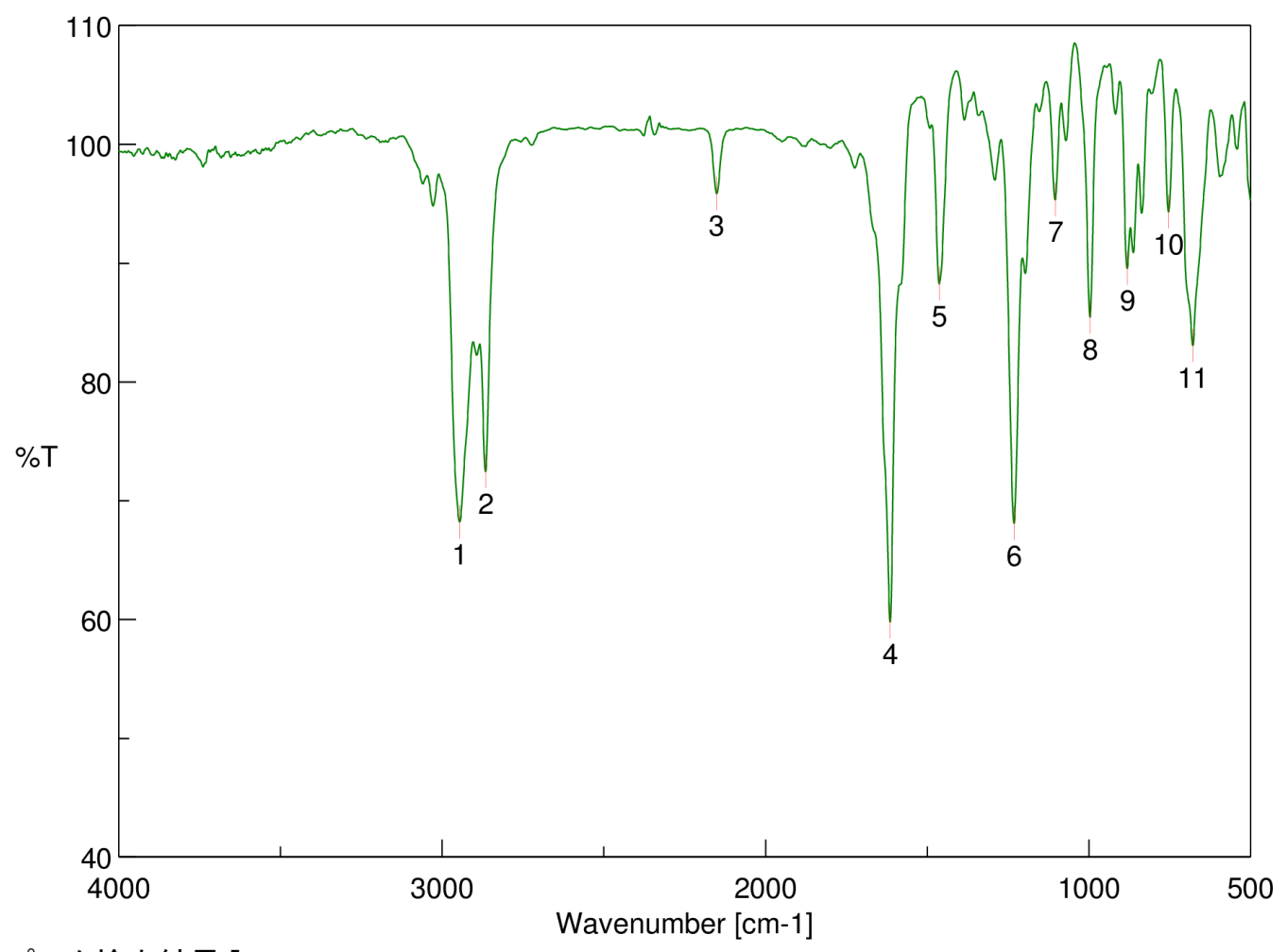

[ピーク検出結果 ]

$\begin{array}{llllll}\text { No. } & \text { 位置 } & \text { 強度 } & \text { No. } & \text { 位置 } & \text { 強度 } \\ 1 & 2945.73 & 68.2095 & 2 & 2865.7 & 72.4437 \\ 3 & 2151.2 & 95.8311 & 4 & 1615.09 & 59.7909 \\ 5 & 1462.74 & 88.2471 & 6 & 1231.33 & 68.0951 \\ 7 & 1104.05 & 95.3236 & 8 & 997.017 & 85.4369 \\ 9 & 881.309 & 89.5574 & 10 & 754.031 & 94.2852\end{array}$<smiles>O=C(C#CS)C=CC=Cc1ccccc1</smiles> 


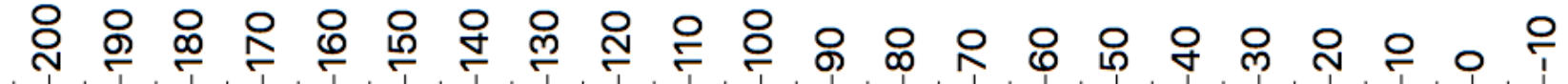

's96'

$796^{\circ} 0$

$066^{\circ} 0$

$\nabla 00^{\circ} \mathrm{L}$

$\varepsilon Z 0^{\circ}\llcorner$

sEO

๕乙Ъ'て

Eカt'て

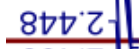

ESt
$0 \angle t \tau$

$9 \angle t Z$

Z8४ Z

s6t' 2

LOS'Z

$\varepsilon \angle 6^{\circ} Z_{7}$

$\angle 86^{\circ} Z$

$000^{\circ} \varepsilon$

$9 Z 0^{\circ} \varepsilon$

$6 \varepsilon 0^{\circ} \varepsilon$

乙९० $\varepsilon$.

6є७' $\varepsilon$

6ยt $\varepsilon$

$0 \angle \nabla \cdot \varepsilon$

乙8॰ $\varepsilon$

เ6๑ $\varepsilon$

ตOG' $\varepsilon$

SL' $\varepsilon$

o\&s' $\varepsilon-$

$97 G^{\prime} \varepsilon-$

Z99' $\varepsilon$

$6 \angle S^{\circ} \nabla-$

169 ' $t$

$\angle 90^{\circ} \mathrm{L}$

$\mathrm{S} \angle 0^{\circ} \angle$

$8 \angle 0^{\circ} \angle$

$980 \circ$

$8 \varepsilon l: L$

$\varepsilon G l \cdot \angle$

$\angle G l \cdot \angle$

$69 L^{\circ} \angle$

$Z \angle L \angle 7$

$68 L^{\circ} \angle$

$902 \angle$

GटZ $\angle$

乙E乙 $L$

$\varepsilon \sqcup Z L^{\circ} L$

$8 \nabla Z \angle$

ISZ $\angle$ -

$09 Z \angle$

$96 Z \angle$

$\varepsilon L \varepsilon^{\prime} L$

乙Eะ \llcorner

แIZ 6 工

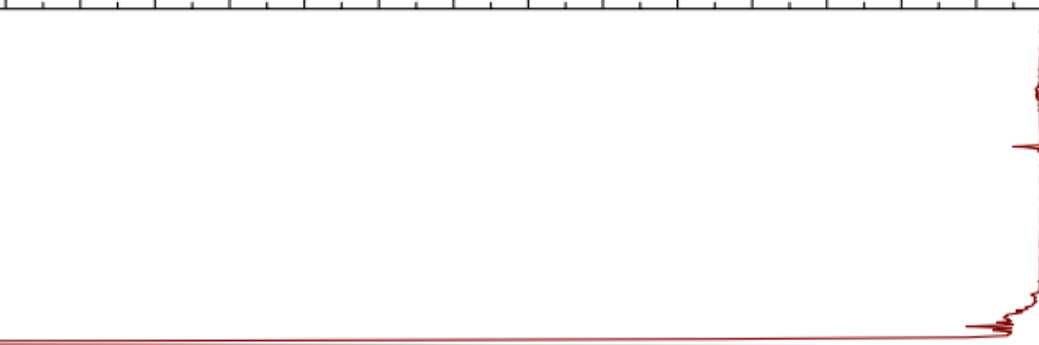

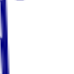

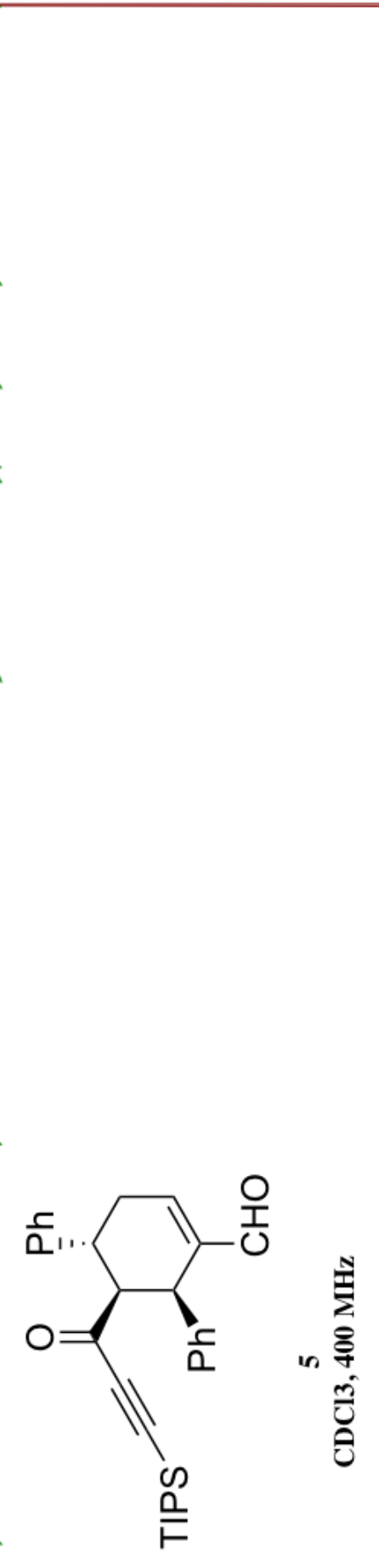

$\varepsilon \angle \nabla^{\circ} 6$ -

LE9 6
9896

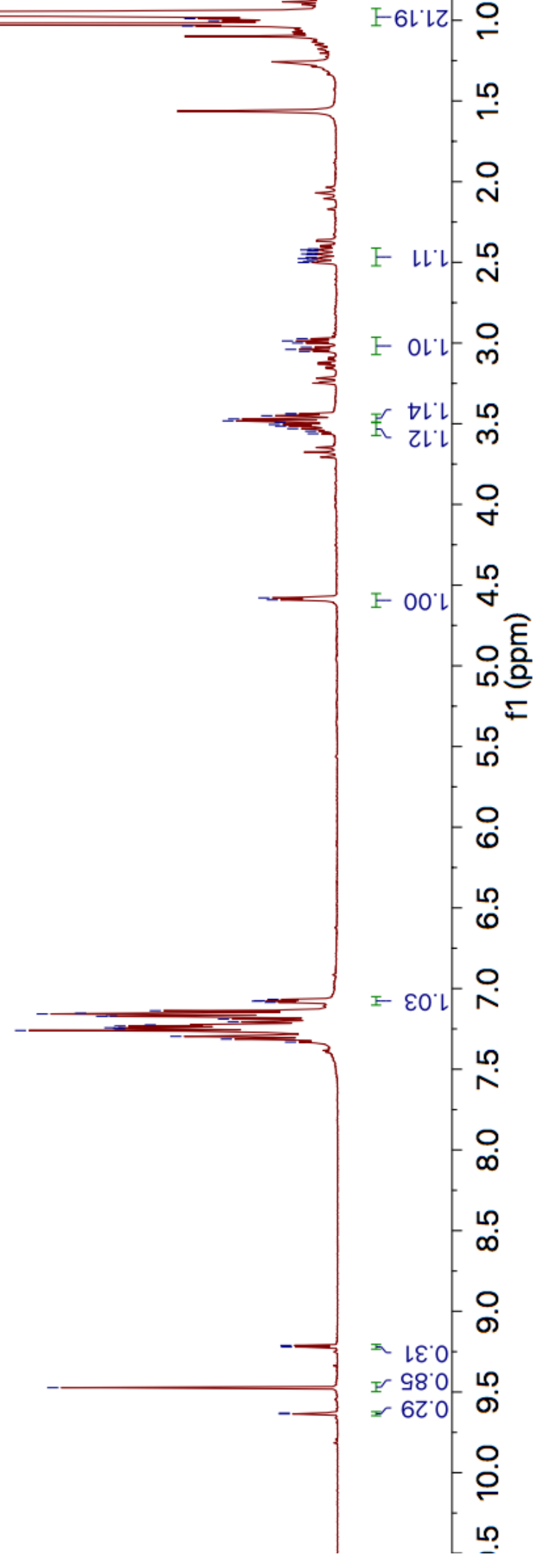




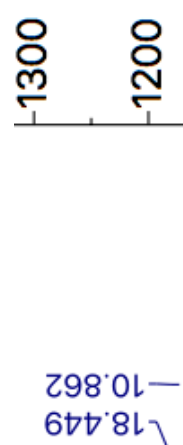

$8 \angle t 81-$

896' $9 \varepsilon$

$299^{\circ} \varepsilon^{-}$

เ8Z゙เ

66L'99-

$189^{\circ} 9 \angle$

$000 \angle L T$

$8 L \varepsilon^{\prime} L L$

8t9' $\angle 6-$

เ๕Z'†OL-

689'9ZL

$10 \varepsilon^{\circ} \angle Z L$

ZLE'8ZL

$Z \nabla \angle B Z L$

$\angle 6 C^{\circ} 6 \mathrm{Zl}^{\circ}$

$\angle L D^{\circ} \angle E L-$

Ot8'LL -

$\angle L \varepsilon^{\prime} \varepsilon L^{-}$

ogl'6tl-

용

ঃ ঃ

\&

웅

요

운 웅 웅

(1) 8

.

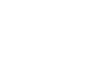




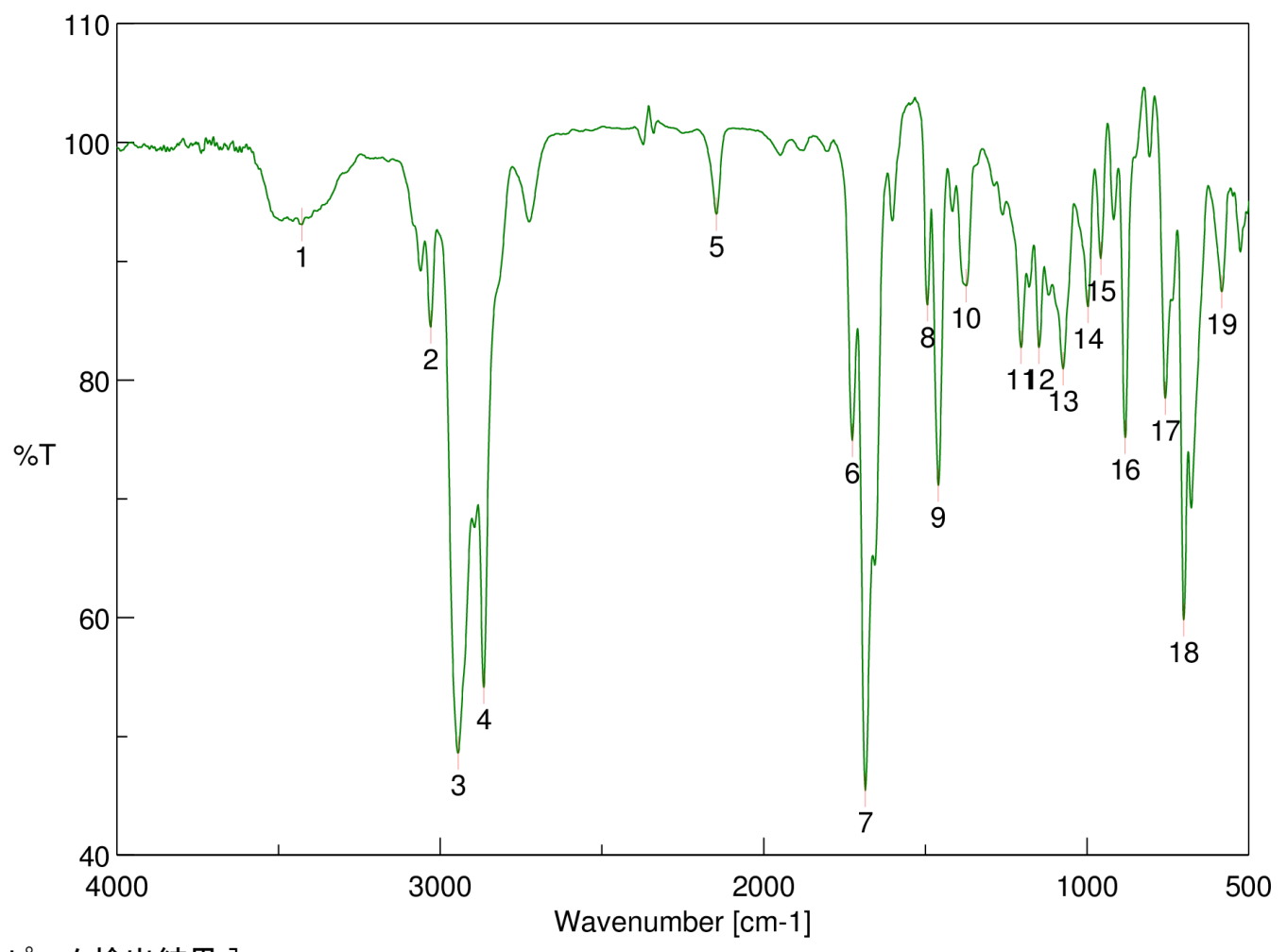

[ピーク検出結果 ]

$\begin{array}{llllll}\text { No. } & \text { 位置 } & \text { 強度 } & \text { No. } & \text { 位置 } & \text { 強度 } \\ 1 & 3427.85 & 93.0799 & 2 & 3029.62 & 84.4673 \\ 3 & 2944.77 & 48.5802 & 4 & 2865.7 & 54.1167 \\ 5 & 2146.38 & 93.9593 & 6 & 1725.98 & 74.888 \\ 7 & 1685.48 & 45.4608 & 8 & 1493.6 & 86.3172 \\ 9 & 1459.85 & 71.1445 & 10 & 1374.03 & 87.9066 \\ 11 & 1204.33 & 82.7268 & 12 & 1148.4 & 82.734 \\ 13 & 1074.16 & 80.9327 & 14 & 997.017 & 86.1704 \\ 15 & 957.484 & 90.2174 & 16 & 882.274 & 75.1639 \\ 17 & 757.888 & 78.4606 & 18 & 700.998 & 59.8131 \\ 19 & 583.361 & 87.4175 & & & \end{array}$

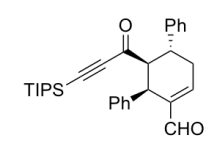

